\title{
Measurement of the $t \bar{t}$ Cross Section Using the Dimuon Channel in $p \bar{p}$ Collisions at $\sqrt{s}=1.96$ $\mathrm{TEV}$ \\ by \\ Robert Crampton McCroskey
}

A Dissertation Submitted to the Faculty of the Department of Physics

In Partial Fulfillment of the Requirements

For the Degree of

Doctor of Philosophy

In the Graduate College

The University of Arizona

2004 
Get the official approval page from the Graduate College before your final defense. 


\section{Statement By Author}

This dissertation has been submitted in partial fulfillment of requirements for an advanced degree at The University of Arizona and is deposited in the University Library to be made available to borrowers under rules of the Library.

Brief quotations from this dissertation are allowable without special permission, provided that accurate acknowledgment of source is made. Requests for permission for extended quotation from or reproduction of this manuscript in whole or in part may be granted by the head of the major department or the Dean of the Graduate College when in his or her judgment the proposed use of the material is in the interests of scholarship. In all other instances, however, permission must be obtained from the author.

SIGNED: 


\section{ACKNOWLEDGMENTS}

I would like to express the deepest thanks to my advisor, Ken Johns, who shows his enthusiasm for the field every day with his work ethic. Ken is a great teacher, leader, and friend whose mentoring made my experience at Fermilab instructional and enjoyable. Special thanks also go to David Fein, for recruiting me and getting me started in the group. Dave's continuing friendship (and pad in Chicago) were a definite plus during my stay at Fermilab.

From Arizona, I want to thank Tommy Burch, Mike Fromerth, and Jason Hamm for their friendship. Thanks to Charles Wolgemuth, who does the best impression of a physicist I have ever seen from a guitar player. Finally, many thanks to Todd Thompson for all of the conversations on the third floor patio.

The list of great people at Fermilab is long and distinguished. In particular, I want to thank John Butler, Darien Wood, Emanuela Barberis, and Dmitri Denisov for support and friendship during my tenure. Noah Wallace, Pete Johnson, Peter Tamburello, and Jessica Levêque made the Arizona office a great place to work and Fermilab a fun place to be. The L1MU crew of Stefan Anderson, Susan Burke and Jason Kasper deserve major thanks for sharing of the difficult job of trigger mainte-

nance and allowing me to finish my thesis. Finally, thanks to Jeff Temple for all of the help in making the trigger run, hard work during the long shutdowns, and taking over the trigger - L1MU is in good hands.

I owe a lot to the hardware types who helped us make a great trigger system. Sten Hansen, Tom Fitzpatrick, Valeri Tokmenin, Petr Neustroev, Jamieson Olsen, and Dan Edmunds all provided us with more help than I can remember. I especially enjoyed working with Mikhail Batouritskiy, who taught me about sweeping out the dark corners and how to say 'short circuit' in Russian. Thanks to Chris Leeman for the amazing work in testing 70 trigger cards. I honestly don't think anyone else could have stuck with that difficult task. Finally, Joel Steinberg deserves major kudos for eternal perseverance with the hardware and infinite patience with the physicists.

Finally, I want to thank my family. Their support and love have helped me make it through the last seven years. Most of all, I want to thank my wife, Shari. Her patience during three long years of travel and late night phone calls has been amazing. It brings me great joy that she was a part of this process with me. 
For my mother, Mary, and in memory of my father, Robert. 


\section{TABle of Contents}

List of Figures $\ldots \ldots \ldots \ldots \ldots \ldots \ldots \ldots \ldots$

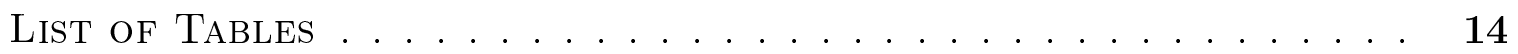

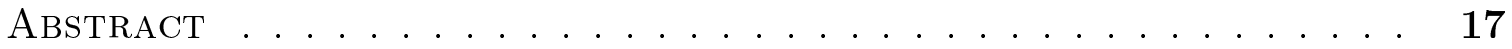

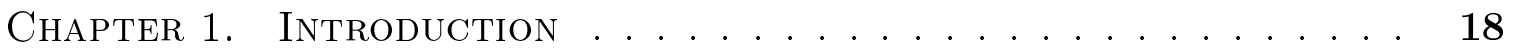

1.1. Standard Model . . . . . . . . . . . . . . . . . . . 18

1.2. The Top Quark . . . . . . . . . . . . . . . . . . 20

1.3. Cross Section Measurement . . . . . . . . . . . . . . . . . 22

1.4. Muon Triggering . . . . . . . . . . . . . . . . 23

Chapter 2. Theory ........................ 24

2.1. Top Quark Pair Production . . . . . . . . . . . . . . . 24

2.1.1. Parton Level Cross Sections . . . . . . . . . . . . . . . 25

2.1.2. Strong Coupling Constant . . . . . . . . . . . . . 26

2.1.3. Parton Distribution Functions . . . . . . . . . . . . . . 28

2.2. Results . . . . . . . . . . . . . . . . . . . . . . 28

2.3. Top Decay . . . . . . . . . . . . . . . . . . . . . . . 31

Chapter 3. Experimental Apparatus . . . . . . . . . . . 34

3.1. Fermilab Tevatron . . . . . . . . . . . . . . . . . 34

3.2. DØ Detector . . . . . . . . . . . . . . . . . . . 37

3.2.1. Silicon Microstrip Tracker . . . . . . . . . . . . . . 38

3.2.2. Central Fiber Tracker . . . . . . . . . . . . . . . . . 40

3.2.3. Preshower Detectors . . . . . . . . . . . . . . . . . 42

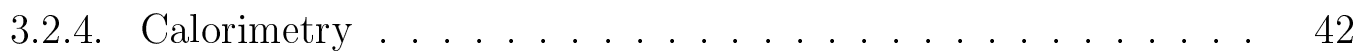

3.2.5. Muon System . . . . . . . . . . . . . . . . . . . 4 45

3.3. DØ Trigger System . . . . . . . . . . . . . . . . . . . . . . . . 49

3.3.1. Level 1 Trigger . . . . . . . . . . . . . . . . . . . 49

3.3.2. Level 2 Trigger . . . . . . . . . . . . . . . . 50

3.3.3. Level 3 Trigger and Data Acquisition . . . . . . . . . . . 51

Chapter 4. Level 1 Muon Trigger . . . . . . . . . . . 52

4.1. Gbit/s Serial Links . . . . . . . . . . . . . . . . . 53

4.2. Octant Trigger Cards . . . . . . . . . . . . . . . 55

4.2.1. Event Synchronization . . . . . . . . . . . . 55

4.2.2. Data Buffering . . . . . . . . . . . . 56 


\section{TABLE of Contents-Continued}

4.3. Trigger Crate . . . . . . . . . . . . . . . . 57

4.4. Trigger Logic . . . . . . . . . . . . . . . 59

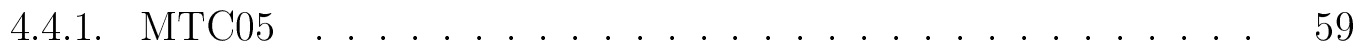

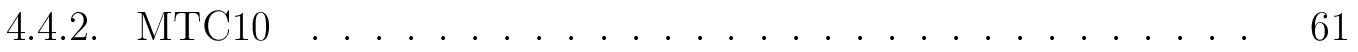

4.4.3. Octant Trigger Counters . . . . . . . . . . . . 63

4.4.4. Crate Manager Triggers . . . . . . . . . . . . . 67

4.4.5. Trigger Manager Logic . . . . . . . . . . . . . . . 67

4.5. Certification . . . . . . . . . . . . . 69

4.6. Performance . . . . . . . . . . . . . . . 70

Chapter 5. Level 1 Muon Operations . . . . . . . . . . 76

5.1. Data Flow . . . . . . . . . . . . . . . 76

5.1.1. Accelerator Timing . . . . . . . . . . . 76

5.1.2. Trigger Cards - Inputs . . . . . . . . . . . . . . 77

5.1.3. Flavor Cards . . . . . . . . . . . . . . . 78

5.1.4. Trigger Cards - Outputs . . . . . . . . . . . . 79

5.1.5. Crate Manager Logic . . . . . . . . . . . . . . . . . 80

5.1.6. Trigger Manager Logic . . . . . . . . . . . . . . . . . 81

5.1.7. Trigger Framework . . . . . . . . . . . . . . 82

5.2. Readout . . . . . . . . . . . . . . . 82

5.2.1. Message Builder . . . . . . . . . . . . . . 83

5.2.2. Muon Readout Crate . . . . . . . . . . . 85

5.3. Control Registers . . . . . . . . . . . . . 86

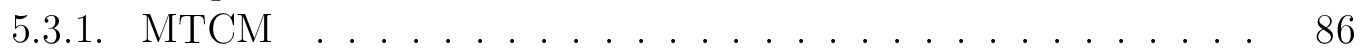

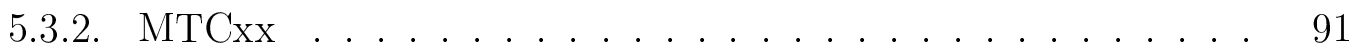

5.4. Status Registers . . . . . . . . . . . . . 95

5.5. Trouble Shooting . . . . . . . . . . . . . . . 96

5.5.1. Trigger Rates . . . . . . . . . . . . . . . 96

5.5.2. Readout .................. . . 97

Chapter 6. Data Selection . . . . . . . . . . . . . . 100

6.1. Data Collection and Processing . . . . . . . . . . . . 100

6.2. Trigger ........................ 101

6.3. Luminosity Measurement . . . . . . . . . . . . . . . 103

6.4. Offline Muon Identification . . . . . . . . . . . . . . . . . 104

6.4.1. Muon Segment and Track Finding . . . . . . . . . . . . 104

6.4.2. Central Track Match . . . . . . . . . . . . . . 106

6.4.3. Muon Quality Cuts . . . . . . . . . . . . . . 106

6.5. Offline Jet Identification . . . . . . . . . . . . . . . 107

6.5.1. Jet Finding . . . . . . . . . . . . . . . . . . . . . . 107 


\section{Table of Contents-Continued}

6.5.2. Jet Corrections . . . . . . . . . . . . . . . . . 108

6.5.3. Jet Quality Cuts . . . . . . . . . . . . . . 109

6.6. Missing Transverse Energy . . . . . . . . . . . . . . . . 110

6.7. Preselection Cuts . . . . . . . . . . . . . . . . 110

6.7.1. Muon Isolation . . . . . . . . . . . . . . . 111

6.7.2. Prompt Muon Cuts . . . . . . . . . . . . . . . 112

6.8. Selection Cuts . . . . . . . . . . . . . . . . 113

6.9. Monte Carlo Samples . . . . . . . . . . . . . . . . . . . . 114

Chapter 7. Efficiencies . . . . . . . . . . . . . . 119

7.1. Cut Order in Analysis Program diMu . . . . . . . . . . . . . 121

7.2. Muon ID . . . . . . . . . . . . . . . . . 123

7.2.1. Muon Reconstruction Data/Monte Carlo Scale Factor . . . . . 123

7.2.2. Track Finding Data/Monte Carlo Factor . . . . . . . . . . . . 124

7.2.3. Muon Isolation Data/Monte Carlo Scale Factor . . . . . . . . 125

7.3. Trigger Efficiency . . . . . . . . . . . . . 126

7.4. Jet ID . . . . . . . . . . . . . . . . . . . . . . . . . . 129

7.5. Prompt Muons . . . . . . . . . . . . . . . . . . 130

7.6. Selection Cuts . . . . . . . . . . . . . 131

7.7. Other Errors . . . . . . . . . . . . . . . . 132

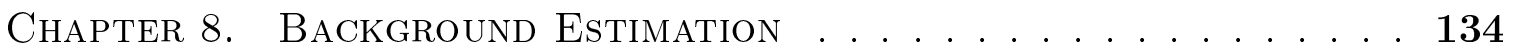

8.1. Background Processes and Monte Carlo Samples . . . . . . . . . . . 134

8.2. $\mathrm{Z} / \gamma^{*} \ldots \ldots \ldots \ldots \ldots \ldots \ldots \ldots \ldots \ldots \ldots$

8.2.1. Raw Yields . . . . . . . . . . . . . 136

8.2.2. Z $/ \gamma^{*}$ Scale Factor . . . . . . . . . . . . . . . . . . 136

8.2.3. Z $/ \gamma^{*}$ Yields . . . . . . . . . . . . . . . . . 138

8.2.4. Z $/ \gamma^{*}$ Reweighting . . . . . . . . . . . . . . 141

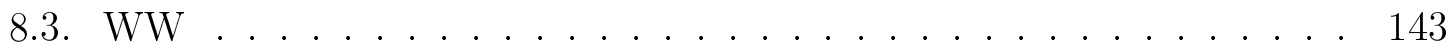

8.4. WQCD Backgrounds .................... 144

8.4.1. WQCD Isolation Efficiency . . . . . . . . . . . . . . . 145

8.4.2. Matrix Method . . . . . . . . . . . . . . 145

8.5. $\mathrm{H}_{T} \mu / E_{T}$ Cut Optimization . . . . . . . . . . . . . . 148

8.6. Data/Monte Carlo Comparisons . . . . . . . . . . . . . . . . 152

8.7. Background Summary . . . . . . . . . . . . . 154

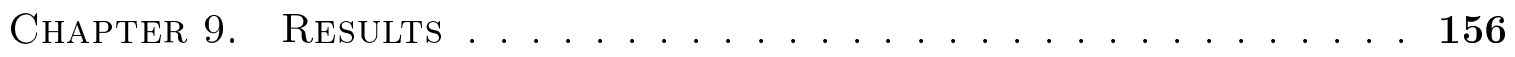

9.1. Cross Section Result . . . . . . . . . . . . . 156

9.2. Systematic Errors . . . . . . . . . . . . . . . . . 159

9.3. Candidate Events . . . . . . . . . . . . . . . 162 
Table of Contents-Continued

9.4. Comparison to Other Measurements . . . . . . . . . . . . . . . 162

9.5. Future Improvements . . . . . . . . . . . . . . . . 168

Chapter 10. Conclusions ................. 170

Appendix A. Level 1 Muon Trigger Inputs . . . . . . . . . . . 173

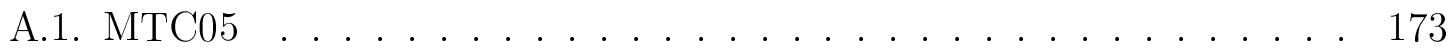

A.1.1. L1CTT Inputs . . . . . . . . . . . . . . . . . . . . . 173

A.1.2. Central Scintillators . . . . . . . . . . . . . . 174

A.1.3. Forward Scintillators . . . . . . . . . . . . . . . 175

A.1.4. MTC05 Inputs . . . . . . . . . . . . . . . . . 176

A.2. $\mathrm{MTC}_{10} \ldots \ldots \ldots \ldots \ldots \ldots \ldots \ldots \ldots \ldots \ldots$

A.2.1. Central . . . . . . . . . . . . . . . 178

A.2.2. Forward . . . . . . . . . . . . . . . . . . . 179

A.2.3. MTC10 Inputs . . . . . . . . . . . . . . . . . . 180

A.3. Splitters . . . . . . . . . . . . . . . . 182

REFERENCES ......................... 184 


\section{List OF Figures}

Figure 1.1. The Standard Model of particle physics. . . . . . . . . . . . . . 19

FIGURE 2.1. Leading order Feynman diagram for $q \bar{q}$ annihilation production of $t \bar{t}$ pairs. . . . . . . . . . . . . . . .

Figure 2.2. Leading order Feynman diagrams for gluon-gluon fusion production of $t \bar{t}$ pairs. . . . . . . . . . . . . . . . . .

FiguRE 2.3. The theoretical cross section for $t \bar{t}$ production at $\sqrt{s}=1.96 \mathrm{TeV}$ as a funtion of the top quark mass. The average NNLO result is the solid line, while the dashed lines are the 1PI (upper) and PIM (lower) results.

Figure 2.4. The Feynman diagram for the weak decay of the top quark. . . 32

Figure 3.1. The Fermilab accelerator complex. . . . . . . . . . . . 35

Figure 3.2. The D $\varnothing$ detector. . . . . . . . . . . . . . . . . . . 38

Figure 3.3. The Silicon Microstrip Tracker (SMT). A combination of barrel and disk detectors allow 3D track reconstruction to $|\eta|<3.0 \ldots \ldots . .39$

Figure 3.4. The CFT detector. . . . . . . . . . . . . . . . . . . . . 41

Figure 3.5. The D $\emptyset$ calorimeter. . . . . . . . . . . . . . . . . 43

Figure 3.6. The muon detector. . . . . . . . . . . . . . . . . . . 46

Figure 4.1. The Level 1 Muon trigger system. . . . . . . . . . . . . . 53

Figure 4.2. The eye pattern before and after the amplifier/equalizer circuit. 54

Figure 4.3. Level 1 Muon trigger crate. . . . . . . . . . . . . . . . . 58

Figure 4.4. MTC05 trigger logic. . . . . . . . . . . . . . . 60

FiguRE 4.5. MTC10 trigger logic. . . . . . . . . . . . . . 63

FiguRE 4.6. The acceptance of the $\mathrm{D} \varnothing$ muon detector for medium muons. The turn-on curve in $\mathrm{P}_{\mathrm{T}}$ is from low energy muons that do not make it through the toroid, while the dips in $\eta$ and $\phi$ come from the limited coverage in the central bottom of the detector. . . . . . . . . . 71

Figure 4.7. The Monte Carlo efficiency for the tight scintillator only and tight scintillator/loose wire trigger. . . . . . . . . . . . . 73

Figure 4.8. The efficiency for the tight scintillator only and tight scintillator/loose wire trigger from data. . . . . . . . . . . . 75

FiguRE 5.1. The accelerator timing for one turn. Data are sent to the L1MU trigger for the 142 bunch crossings outside of the Synch Gap. During the Synch Gap, idle characters are sent allowing the input FIFO's to go empty. 77

Figure 5.2. Data and timing signals sent to the flavor card. The startprocessing signal is the same as input-ready and is high the entire time data are present. BC-clock marks the first of seven $18.8 \mathrm{~ns}$ strobes of data to the flavor card. The data are labeled as bunch crossing/strobe. . . . . 


\section{List of Figures-Continued}

FiguRE 5.3. Timing of the trigger decisions from the flavor card. The 36 bits of trigger information for $\mathrm{BC} 7$ are sent from the flavor card and are divided into three groups of 12 bits. The multiplexed trigger decisions are read by the MTCM across the backplane and summed for the eight octants. . . . . . . . . . . . . . . . . . .

FIGURE 5.4. A schematic view of the readout path for the north trigger crates. All trigger information originates with the Trigger Framework and is distributed to the Geographic Sections over the Serial Command Link (SCL). Upon receiving a Level 2 Accept, the front ends send data to the Muon Readout Cards, which are then readout by the Single Board Computer and sent to the Level 3 farm nodes. . . . . . . . . . . . . . .

Figure 6.1. An event display of a Monte Carlo muon showing the wire hits, segments, and scintillator hits. . . . . . . . . . .

Figure 6.2. Dimuon invariant mass versus $E_{T}$ for $\mathrm{Z} / \gamma^{*} \mathrm{MC}$ without the $\Delta \phi$ cut (left) and with. Events with poorly reconstructed muons tend to have large invariant mass and $\mathbb{E}_{T} \ldots \ldots \ldots \ldots \ldots \ldots$

Figure 6.3. The invariant mass of dimuon events in data. The fit shown is a combined Gaussian and exponential. The mean and width of the Gaussian are $89.1 \mathrm{GeV} / \mathrm{c}^{2}$ and $8.9 \mathrm{GeV} / \mathrm{c}^{2}$, respectively. The arrows indicate the chosen cuts. . . . . . . . . . . . . . . . . . . .

Figure 7.1. The Level 1 and Level 2 single muon trigger efficiency parameterizations used in determining the overall trigger efficiency for Monte Carlo events. . . . . . . . . . . . . . . . . . .

Figure 7.2. The $\Delta \phi\left(\mu_{\text {leading }}, E_{T}\right)$ and $\mathrm{H}_{T} \mu$ distributions for signal Monte Carlo. The regions excluded by the cuts are indicated by the shaded

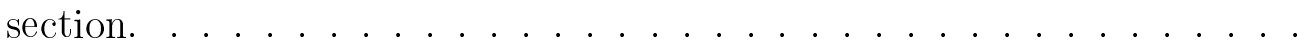

Figure 7.3. The mass and $E_{T}$ distributions for signal Monte Carlo. The regions excluded by the cuts are indicated by the shaded section. . . . .

Figure 8.1. The data/Monte Carlo scale factor $\kappa_{Z}$ as a function of $\mathrm{H}_{T} \mu$, left, and $\not_{T}$, right. The line is drawn at the chosen value for $\kappa_{Z}$ of 0.781 . .

Figure 8.2. Comparison of the data (points) and Monte Carlo (histogram) distributions for the leading and second jet $\mathrm{E}_{\mathrm{T}}$. The data have more events at low $\mathrm{E}_{\mathrm{T}} \ldots \ldots \ldots \ldots \ldots \ldots \ldots \ldots$

Figure 8.3. Leading muon $\mathrm{P}_{\mathrm{T}}, \mathrm{H}_{T} \mu$, dimuon invariant mass, and the $\mathrm{Z} \mathrm{P}_{\mathrm{T}}$ spectrum for $\mathrm{Z}$ control events in data and Monte Carlo. . . . . . . . .

Figure 8.4. The event weights for the leading and second jet as a function of the jet $\mathrm{E}_{\mathrm{T}} \ldots \ldots \ldots \ldots \ldots \ldots \ldots \ldots \ldots \ldots$

FIGURE 8.5. Isolation efficiency for WQCD events in data as a function of $E_{T}$. A fit to the low $\not_{T}$ region gives $\epsilon_{W Q C D}=0.049 \ldots \ldots \ldots \ldots$ 


\section{List of Figures-Continued}

Figure 8.6. Scatter plots for $\mathrm{H}_{T} \mu$ versus $E_{T}$ for $t \bar{t}$ (left) and $\mathrm{Z} / \gamma^{*}$ mass 2 Monte Carlo (right). . . . . . . . . . . . . . . . . . . 149

FiguRE 8.7. Plot of the inverse of the FOM for various values of the $E_{T}$ and $\mathrm{H}_{T} \mu$ cut. The plot on the left shows the values of the FOM, with the maximum of 0.51 reached at a $\mathbb{E}_{T}$ cut of $45 \mathrm{GeV}$ and a $\mathrm{H}_{T} \mu$ cut of 120 $\mathrm{GeV}$ (some adjacent values have the same FOM). The plot on the right shows contours for the FOM in the $E_{T}-\mathrm{H}_{T} \mu$ plane. . . . . . . . . . . .

FIGURE 8.8. Plot of the expected signal yield as a function of the expected background $\left(\mathrm{Z} / \gamma^{*}\right.$ and WW events) yield for various values of the $E_{T}$ and $\mathrm{H}_{T} \mu$ cut. The chosen cut of $E_{T}>35 \mathrm{GeV}$ and $\mathrm{H}_{T} \mu>120 \mathrm{GeV}$ is shown

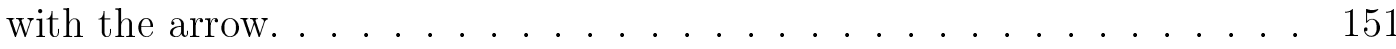

Figure 8.9. Leading muon $\mathrm{P}_{\mathrm{T}}$ (left) and $|\eta|$ (right). . . . . . . . . . . . 153

Figure 8.10. Leading jet $\mathrm{E}_{\mathrm{T}}$ (right) and $\mathrm{H}_{T} \mu$ (left). . . . . . . . . . . 153

Figure 8.11. Dimuon invariant mass (left) and $\mathbb{F}_{T}$ (right). The arrows indicate the selection cuts. . . . . . . . . . . . . . 154

FigurE 9.1. The negative log-likelihood function for the cross section result.

Figure 9.2. The $68 \%$ CI values for $\mathrm{N}_{\text {sig }}$ with $\mathrm{N}_{b k g}$ equal to 2.61 events for various values of $\mathrm{N}$. Also shown is the measured number of data events $\mathrm{N}$ of four. . . . . . . . . . . . . . . . . .

FigurE 9.3. The theoretical NNLO average cross section (solid line) with errors (dashed line). The data point is the measured value of $11.1 \mathrm{pb}$ at a top mass of $178 \mathrm{GeV}$. The inner error bar is statistical only, the outer is statistical and systematic combined. . . . . . . . . . .

FiguRE 9.4. The muon display for one of the candidate events, viewed along the beam axis. . . . . . . . . . . . . . . . . 164

FIGURE 9.5. The central tracker and calorimeter, viewed along the beam axis. The hits in the muon system are represented by the rectangles. High $\mathrm{P}_{\mathrm{T}}$ tracks, shown as straight lines, can be seen in the central tracker extending to the muon hits. . . . . . . . . . . . . . . . 165

Figure 9.6. The calorimeter lego display for event $178423 / 35258885$. The leading jet has an $\mathrm{E}_{\mathrm{T}}$ of $144 \mathrm{GeV}$, while the second jet has an $\mathrm{E}_{\mathrm{T}}$ of $43 \mathrm{GeV} .166$

Figure 9.7. Comparison of this measurement (DØ Run II Dimuon) to the Run I DØ and CDF dilepton measurements and to the Run II CDF dilepton measurement. The DØ Run II dilepton result is also shown and includes this measurement and is from the Winter 2004 conference results. 167

Figure A.1. MTCC 05 inputs. . . . . . . . . . . . . . . 176

Figure A.2. MTCN 05 inputs. . . . . . . . . . . . . . . . . 177

Figure A.3. Central MTC10 inputs. . . . . . . . . . . . . 180

Figure A.4. Forward MTC10 inputs. . . . . . . . . . . . . . . 181 
List of Figures-Continued

Figure A.5. CTT Splitters. . . . . . . . . . . . . . . . . . . 182

Figure A.6. Central/North Splitters. . . . . . . . . . . . . . . 183

Figure A.7. South Splitters. . . . . . . . . . . . . . . . . . . . 183 


\section{LIST OF TABLES}

TABLE 1.1. The four forces in nature with their relative strengths and gauge

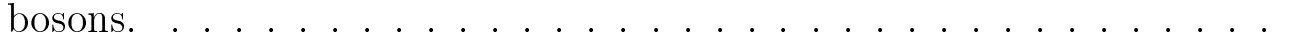

TABLE 2.1. Cross section results for $\mathrm{m}_{t}=175 \mathrm{GeV}$. The 1PI and PIM results are NNLO calculations $[11] \ldots \ldots \ldots \ldots \ldots \ldots$

TABLE 2.2. The final states for $t \bar{t}$ decay, classified by the decay of the $\mathrm{W}$ bosons. The bottom quarks from the decay of the top quark and the light quarks from the decay of the $\mathrm{W}$ boson appear as jets in the detector and are denoted as $j$ in the final state. The dimuon channel has a branching

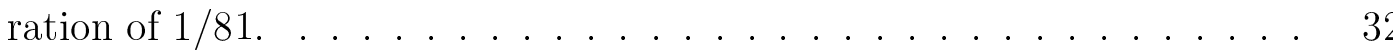

TABLE 3.1. Silicon Microstrip Tracker design parameters. . . . . . . . . . . 40

TABLE 4.1. MTC05 trigger latency. . . . . . . . . . . . . . . 62

TABLE 4.2. CF MTC10 trigger latency. . . . . . . . . . . . . . . . . 64

TABLE 4.3. EF MTC10 trigger latency. . . . . . . . . . . . . . . . . 64

TABLE 4.4. Central 2-bit octant trigger decision counters. . . . . . . . . 65

TABLE 4.5. Forward 2-bit octant trigger decision counters. . . . . . . . . 66

TABLE 4.6. Details of the loose and tight triggers for the central and forward regions. Additional details can be found in [38]. . . . . . . . . . 66

TABLE 4.7. Central MTC05/MTC10 combined triggers. . . . . . . . . . . 68

TABLE 4.8. Forward MTC05/MTC10 combined triggers. . . . . . . . . . 68

TABLE 5.1. Map of the nine L1MU trigger front end crates to the readout crate and section. . . . . . . . . . . . . .

TABLE 5.2. A summary of the registers set on the MTCM, the values to which they are set, and the CPLD that implements the register. . . . . . . .

TABLE 5.3. Bus grant control for the MTCM. Setting a given bit high to this register will enable the given section access to the VME bus. . . . . . 87

TABLE 5.4. MTCM trigger logic settings used to enable octant triggers. Setting a bit high will include a given octant in the trigger. . . . . . . . . 91

TABLE 5.5. MTCxx control masks. . . . . . . . . . . . . . . . 92

TABLE 5.6. Auxiliary control and status register. . . . . . . . . . 95

TABLE 5.7. Serial link status registers. Each register has 16 bits, one for each serial link on the MTCxx. . . . . . . . . . . . . . 95

TABLE 6.1. The integrated luminosity for the five versions of the global trigger list after all run quality cuts have been applied. . . . . . . . . . . . . 101

TABLE 6.2. Trigger requirements. . . . . . . . . . . . . . . . . . 102 


\section{List of TABLES-Continued}

TABLE 6.3. Details of the Monte Carlo samples used in this analysis. The $\mathrm{Z} / \gamma^{*}$ Monte Carlo was divided into three samples based on the invariant mass of the lepton pair. The mass ranges were 15 to $60 \mathrm{GeV} / \mathrm{c}^{2}, 60-130 \mathrm{GeV} / \mathrm{c}^{2}$, and $>130 \mathrm{GeV} / \mathrm{c}^{2}$. A signal $t \bar{t}$ sample with identical parameters but with the 'TuneA' multiple parton interactions included was also generated. . .

TABLE 6.4. Details of Monte Carlo samples used for muon reconstruction efficiency studies (Lepton + jets $t \bar{t}$ ) and track finding efficiency studies $\left(\right.$ PYTHIA Z $\left./ \gamma^{*} \rightarrow \mu \mu\right) \ldots \ldots \ldots \ldots \ldots \ldots$

TABle 6.5. Z boson decays in Z $/ \gamma^{*}$ Monte Carlo. . . . . . . . . . . . . . 117

TABLE 6.6. Monte Carlo samples used for this analysis with the Monte Carlo cross section times branching fraction and the number of generated events. For the $\mathrm{Z} / \gamma^{*}$ events, the value for the ALPGEN $\sigma \times \mathrm{BR}$ is the cross section times branching fraction for $\mathrm{Z} / \gamma^{*} \rightarrow$ ee. The combined cross section is the ALPGEN cross section times the conversion factor $\kappa$ of 2.123, which accounts for the five decay channels in the sample (Equation 6.9). . . .

TABLE 7.1. Breakdown of efficiencies for $t \bar{t} \rightarrow \mu \mu$ events (where the muon is allowed to come from $W$ or $\tau$ ) and the corresponding scale factors to account for the difference in efficiency measured in data and Monte Carlo. Errors are statistical only. . . . . . . . . . . . . . . . . . . 120

TABLE 7.2. List of cut numbers in the analysis program diMu . . . . . . . 121

TABLE 7.3. Summary of analysis program diMu cut numbers used for the various efficiencies listed in Table 7.1. . . . . . . . . . . . . . 122

TABLE 7.4. Summary of the relative systematic uncertainties on the $t \bar{t} \rightarrow \mu \mu$

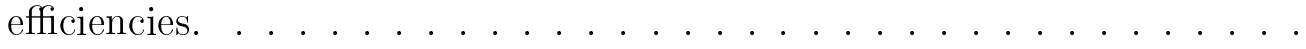

TABLE 8.1. Estimated $\mathrm{Z} / \gamma^{*}$ yields from Monte Carlo before the data/Monte Carlo normalization. The $\mathrm{Z} / \gamma^{*}$ Monte Carlo samples have been tagged as the direct $\mathrm{Z} / \gamma^{*} \rightarrow \mu \mu$ decay or the cascade decay $\mathrm{Z} / \gamma^{*} \rightarrow \tau \tau \rightarrow \mu \mu$ and represent a fraction of the total Monte Carlo sample described in Section 6.9. The decay $\mathrm{Z} / \gamma^{*} \rightarrow \mu \mu$ accounts for roughly $95 \%$ of the estimated

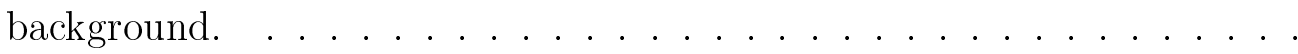

TABle 8.2. The number of $\mathrm{Z} / \gamma^{*}$ mass2 Monte Carlo events, expected Monte Carlo yield, and the number of data events in the $\mathrm{Z}$ control sample. The mass1 and mass3 $\mathrm{Z} / \gamma^{*}$ Monte Carlo contribute less that 0.1 events and are ignored. The scale factor, $\kappa_{Z}$, is the ratio of the data yield to the Monte Carlo yield. . . . . . . . . . . . . . . . . .

TABLE 8.3. Estimated $\mathrm{Z} / \gamma^{*}$ yields from Monte Carlo, scaled to data. The data/Monte Carlo normalization factor is $0.781 \ldots \ldots \ldots \ldots \ldots$

TABLE 8.4. Z $/ \gamma^{*}$ yields for the default Jet Energy Scale, and with the JES increased and decreased by $1 \sigma \ldots \ldots \ldots \ldots \ldots 14 \ldots \ldots$ 


\section{List OF TABLES-Continued}

TABLE 8.5. The reweighted scale factor from the $\mathrm{Z}$ control sample. . . . . . . 142

TABLE 8.6. Estimated reweighted $\mathrm{Z} / \gamma^{*}$ yields from Monte Carlo, scaled to data. The reweighted data/Monte Carlo normalization factor is 0.638. . 143

TABLE 8.7. Estimated WW yields from Monte Carlo for the default jet energy scale and with the jet energy scale increased and decreased by one sigma. 143

TABLE 8.8. Statistical and systematic errors on all terms in the calculation. . 155

TABLE 8.9. Summary of the relative systematic uncertainties on the backgrounds. The total error is from the propagation of all systematic errors on the total background (Table 8.8) . . . . . . . . . . . . 155

TABLE 9.1. Final cross section parameters and result. . . . . . . . . . 156

TABLE 9.2. Statistical and systematic errors on all terms in the calculation. . 160

TABLE 9.3. Summary of the relative systematic uncertainties on the $t \bar{t} \rightarrow \mu \mu$ efficiencies. The last row is the total error without the jet energy scale errors, which is used for the WW background. . . . . . . . . . . . 162

TABLE 9.4. Kinematic variables for the muons, jets, and $E_{T}$ for the four candidate events in data. . . . . . . . . . . . . .

TABLE 9.5. Summary of the number of data events, estimated background, and the signal efficiency for this measurement (D $\varnothing$ Run II), the Run I DØ result, and the Run I and Run II CDF results. Note the CDF results are for the combined dilepton results in the ee, $\mathrm{e} \mu$, and $\mu \mu$ channels, which increases the branching ratio by a factor of four. . . . . . . . . . . 168

TABLE A.1. CTT data formats to L1MU. The six highest $\mathrm{P}_{T}$ tracks from each sector are sent, along with a parity word for error checking. Each 16-bit word gives the $\phi$ position in the outer $(\mathrm{H})$ layer of the CTT, information about $\mathrm{P}_{T}$, and the curvature in the magnetic field. . . . . . . . 174

TABLE A.2. Central scintillator front end crates. . . . . . . . . . . . . . 174

TABLE A.3. Scintillator data formats to L1MU. . . . . . . . . . . . 175

TABLE A.4. North scintillator front end crates. The south has the same configuration with crate numbers $\mathrm{x} 90, \mathrm{x} 92$, etc. . . . . . . . 176

TABLE A.5. PDT data format for wire hits to L1MU. . . . . . . . . . . 178

TABLE A.6. North MDT front end crates. The south has the same configuration with crate numbers $\mathrm{x} 80, \mathrm{x} 82$, etc. . . . . . . . . . . . 179 


\section{Abstract}

We have measured the $t \bar{t}$ production cross section at $\sqrt{s}=1.96 \mathrm{TeV}$ using data collected by the DØ experiment at Fermilab. The integrated luminosity of the data set is $140 \mathrm{pb}^{-1}$ and a total of four candidate events are seen, with an expected background of 2.61 events. The measured cross section of $\sigma_{t \bar{t}}=11.1_{-9.3}^{+22.1}$ (stat.) ${ }_{-4.5}^{+4.3}$ (sys.) $\mathrm{pb}$ is in aggreement with a NNLO calculation of $6.77 \mathrm{pb}$. 


\section{Measurement of the $t \bar{t}$ Cross Section Using the Dimuon Channel in $p \bar{p}$ Collisions at $\sqrt{s}=1.96 \mathrm{TeV}$}

\section{Robert Crampton McCroskey, Ph.D.}

The University of Arizona, 2004

Director: Kenneth Johns

We have measured the $t \bar{t}$ production cross section at $\sqrt{s}=1.96 \mathrm{TeV}$ using data collected by the $\mathrm{D} \emptyset$ experiment at Fermilab. The integrated luminosity of the data set is $140 \mathrm{pb}^{-1}$ and a total of four candidate events are seen, with an expected background of 2.61 events. The measured cross section of $\sigma_{t \bar{t}}=11.1_{-9.3}^{+22.1}$ (stat.) ${ }_{-4.5}^{+4.3}$ (sys.) pb is in aggreement with a NNLO calculation of $6.77 \mathrm{pb}$. 


\section{CHAPTER 1 \\ INTRODUCTION}

Particle physics seeks to identify the smallest building blocks in matter and how these particles interact with each other. We presently believe these building blocks to consist of six quarks, six leptons, and the gauge bosons that mediate the interactions between them. The six leptons include three that have an electrical charge (the electron, muon, and tau) and three associated particles that are neutral, called neutrinos. The quarks, unlike the leptons, do not exist freely in nature and are always bound to other quarks in particles called hadrons, which include protons and neutrons. The current theory that governs these particles is called the Standard Model.

\subsection{Standard Model}

The six quarks and six leptons in the Standard Model are arranged in three generations, with the particles in the second and third generations identical to those in the first except more massive (Figure 1.1). Every particle has an antiparticle, identical except for opposite charge and other quantum numbers. Interactions between the quarks and leptons are mediated by the gauge bosons, which include the photon (electromagnetic interactions), the $\mathrm{W}$ and $\mathrm{Z}$ bosons (weak interactions), and gluons (strong interactions).

The Standard Model is a combination of the Electroweak (EW) theory, which combines the electromagnetic and weak interactions, and Quantum Chromodynamics (QCD), which governs the strong interaction. Both are examples of gauge theories,

meaning they are invariant under local gauge transformations. The EW theory is based on an $\mathrm{SU}(2)_{L} \otimes \mathrm{U}(1)_{Y}$ gauge group, where the generators of the $\mathrm{SU}(2)_{L}$ trans- 


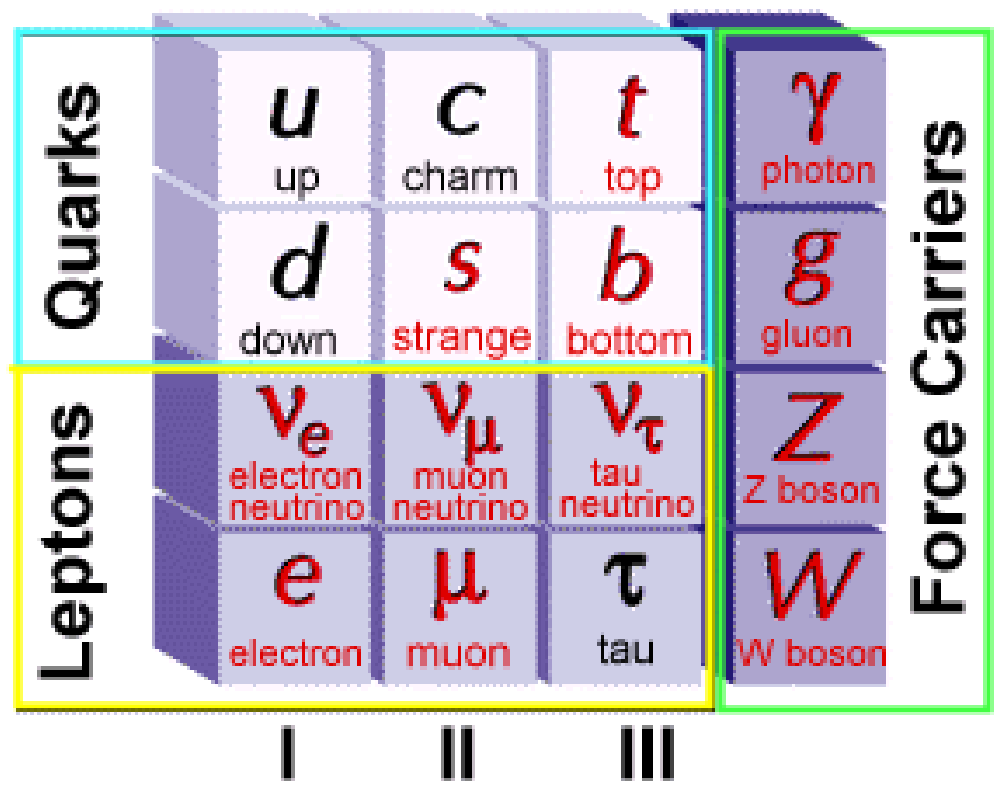

Figure 1.1. The Standard Model of particle physics.

formations are called the Weak Isospin, $\mathrm{T}$, and the generator of the $\mathrm{U}(1)_{Y}$ gauge transformation is the Weak Hypercharge, Y. A total of four gauge bosons are present in the EW theory, three from the $\mathrm{SU}(2)_{L}$ group and one from the $\mathrm{U}(1)_{Y}$ group. At low energies, the symmetry of this group is spontaneously broken via the Higgs mechanism, giving three of the bosons $\left(\mathrm{W}^{ \pm}\right.$and $\mathrm{Z}$ ) mass and leaving the fourth (the photon) massless. In addition, the fermions can couple to the Higgs field, giving them mass as well.

Quantum Chromodynamics (QCD) governs the strong force felt by quarks and is based on an $\mathrm{SU}(3)_{C}$ group, where the generators of the $\mathrm{SU}(3)_{C}$ transformations are called color. A total of eight massless gauge bosons, called gluons, exist in QCD and carry the color charge. Table 1.1 gives the relative strengths of the electromagnetic, weak, and strong forces in the Standard Model and the gauge boson that mediates the force. The fourth force, gravity, is also listed, but is not described by the Standard 


\begin{tabular}{|l|l|l|}
\hline Force & Coupling Strength & Gauge Boson \\
\hline Gravity & $10^{-38}$ & Graviton \\
\hline Weak & $10^{-5}$ & $\mathrm{~W}^{+}, \mathrm{W}^{-}$, and $\mathrm{Z}^{0}$ \\
\hline Electromagnetic & $1 / 137$ & Photon \\
\hline Strong & 1 & Gluon \\
\hline
\end{tabular}

TABLE 1.1. The four forces in nature with their relative strengths and gauge bosons.

Model. A detailed summary of the Standard Model can be found in [1].

The Standard Model successfully predicts many experimentally measurable quantities, such as the anomalous magnetic moments of the electron and the muon, the masses of the $\mathrm{W}$ and $\mathrm{Z}$ bosons, and production cross sections and decay rates. A recent review of precision Standard Model tests in EW measurements shows very good agreement with experiment for the parameters in the Standard Model [2].

There are, however, some problems with the Standard Model. The large number of arbitrary parameters, including all fermion masses, and the large number of particles make it unsatisfying as a final theory. Attempting to extrapolate the Standard Model to Grand Unified Theory, or GUT, energy scales at which EW and QCD interactions have the same strength (around $10^{16} \mathrm{GeV}$ ) gives rise to the hierarchy problem. A concrete example of this problem is the mass of the Higgs boson, where radiative corrections to the mass lead to quadratic divergences in $\Lambda$, the energy scale of new physics [2] [3]. A light Higgs mass (less than $1 \mathrm{TeV}$ ) requires fine-tuning of the parameters to enormous precision.

\subsection{The Top Quark}

The top quark was discovered at Fermilab in 1995 by the D $\varnothing$ and CDF collaborations and completed the search for the sixth and likely final quark in the Standard Model [4] [5]. The current combined (D $\varnothing$ and CDF) measurement of the mass is $178 \pm 4.3$ $\mathrm{GeV} / \mathrm{c}^{2}$, making the top quark about 35 times heavier than the next heaviest, the 
bottom quark [6].

The top quark is produced at the Tevatron primarily as top-antitop $(t \bar{t})$ quark pairs via the strong interaction. In Run I, at a center of mass energy of $\sqrt{s}=1.8 \mathrm{TeV}, \mathrm{D} \varnothing$ measured a value for the $t \bar{t}$ production cross section of $5.7 \pm 1.6 \mathrm{pb}$, while the Run I CDF value is $6.5_{-1.4}^{+1.7} \mathrm{pb}$ [7] [8]. The largest source of error for both experiments is the statistical error associated with the relatively small data sets. Both results agree within one standard deviation to theoretical predictions from the Standard Model.

Run II at the Fermilab Tevatron is an opportunity to extend the measurements of the top quark. The expected data set will be about 40 times larger, allowing precision measurements of various top quark properties. A precision measurement of the top quark mass, along with a measurement of the $\mathrm{W}$ boson mass, constrains the mass of the Higgs boson. Because the top quark decays before hadronization, measurements of spin correlations between the $t \bar{t}$ pair can be made as well as a measurement of the helicity of the $\mathrm{W}$ bosons from the top decay and compared to Standard Model predictions.

The measurement of the production cross section in Run II is important for many reasons. Measuring the cross section is a fundamental test of Standard Model predictions. Improved cross section calculations have been done to next-to-next-to leading order (NNLO) and can be compared to the measured value. The center of mass energy for Run II has increased from 1.8 to $1.96 \mathrm{TeV}$, which gives an increase in the theoretical prediction for the cross section of about 30\% that can be measured.

In addition to testing the Standard Model, precision measurements of the $t \bar{t}$ cross section can help distinguish between various theories beyond the Standard Model. One such theory, called topcolor, attempts to explain the large top quark mass by proposing additional coupling to the third generation quarks [9]. In topcolor, a new neutral gauge boson, called the $\mathrm{Z}^{\prime}$, exists that couples to the top quark and decays to a $t \bar{t}$ pair. Production of $\mathrm{Z}^{\prime}$ bosons would show up as an enhancement of the $t \bar{t}$ cross section over the Standard Model prediction. Calculations at $\sqrt{s}=1.8 \mathrm{TeV}$ show 
that for certain values of the $Z^{\prime}$ mass, the cross section can be enhanced by several picobarns [9]. Other extensions to the Standard Model that involve the top quark are reviewed in $[10]$.

\subsection{Cross Section Measurement}

The top quarks produced decay almost $100 \%$ of the time to a real $\mathrm{W}$ boson and a bottom quark. This analysis measures the $t \bar{t}$ production cross section in events where both $\mathrm{W}$ bosons from the decay of the $t \bar{t}$ pair decay to a muon and a muon neutrino. This decay channel has a relatively small branching fraction of $1 / 81$, but the presence of two high energy muons, energetic jets (from the bottom quarks), and missing energy (from the neutrinos) in the final state keep the expected background relatively low.

The cross section, $\sigma_{t \bar{t}}$, is related to the production rate $R$ by

$$
R=\sigma_{t \bar{t}} \times \mathcal{L}
$$

where $\mathcal{L}$ is the luminosity, measured as the number of particles (protons and antiprotons) per unit area per time. The total number of $t \bar{t}$ 's produced is simply the cross section times the total, or integrated, luminosity.

The method used in this analysis is to identify a series of selection criteria, or cuts, which select likely signal events based on the signature in the detector while minimizing background events. The efficiency for a given cut is defined as the fraction

of signal or background events that remain after the cut has been applied. Once all cuts have been applied, the expected number of background events is subtracted from the number of data events to get a measured number of signal events in data. The number of signal events is combined with the total signal efficiency and the integrated luminosity for the data set to extract the cross section. 


\subsection{Muon Triggering}

The Level 1 Muon (L1MU) trigger is part of the first of three stages of online event filtering. This filter, or trigger, reduces the event rate from $1.7 \mathrm{MHz}$ to the $50 \mathrm{~Hz}$ that can be written to tape for offline analysis. The L1MU trigger uses custom electronics to process 750 Gbits of data every second, selecting events with hits in the muon system consistent with one or two muons. This analysis uses the dimuon trigger at Level 1, which is over $90 \%$ efficienct at selecting events with two or more high $\mathrm{P}_{\mathrm{T}}$ muons.

The University of Arizona is responsible for the design, operation, and performance of the L1MU trigger. A description of the hardware, including the Gbit/s serial links used for data transfer and the custom Field Programmable Gate Arrays (FPGA's) that perform the filtering is given in Chapter 4. Details of the operation

of L1MU trigger, including the control and monitoring of the system, are given in Chapter 5. 


\section{CHAPTER 2}

\section{THEORY}

\subsection{Top Quark Pair Production}

The top quark is produced in $p \bar{p}$ collisions at the Fermilab Tevatron at a total center of mass energy of $\sqrt{s}=1.96 \mathrm{TeV}$. The collisions of interest are between the partons (quarks and gluons) that comprise the protons and antiprotons. Each parton (i,j) carries some fraction $x_{i, j}$ of the total proton or antiproton momentum. The total center of mass energy squared for a given parton interaction is then

$$
\hat{s}=x_{i} x_{j} s
$$

To calculate the total cross section, $\sigma_{t \bar{t}}$, the cross section for all possible individual parton interactions $i+j \rightarrow t \bar{t}$ is calculated. Next, the full range of possible parton momenta are integrated over. Finally, the contributions from different parton interactions are summed. The general form for the differential cross section can be written as

$$
\sigma(s)=\sum_{i, j} \int d x_{i} d x_{j} \hat{\sigma}_{i j}\left(\hat{s}, m_{t}^{2}, \mu_{1}^{2}\right) F_{i}^{p}\left(x_{i}, \mu_{2}^{2}\right) F_{j}^{\bar{p}}\left(x_{j}, \mu_{2}^{2}\right)
$$

where $\hat{\sigma}_{i j}$ is the parton-level cross section for $i+j \rightarrow t \bar{t}$ (Section 2.1.1), $\hat{s}=x_{A} x_{B} s$ is the center of mass energy squared for the partons, and $m_{t}$ is the mass of the top quark [11]. The factors $F_{i, j}\left(x_{i, j}, \mu_{2}^{2}\right)$ are the Parton Distribution Functions (PDF's), which give the probability that a given parton carries a fraction $\mathrm{x}$ of the total hadron momentum (Section 2.1.3). The factors $\mu_{1}$ and $\mu_{2}$ are the renormalization and factorization scales and are set equal to each other $\left(\mu_{1}=\mu_{2}=\mu\right)$ for this calculation. 


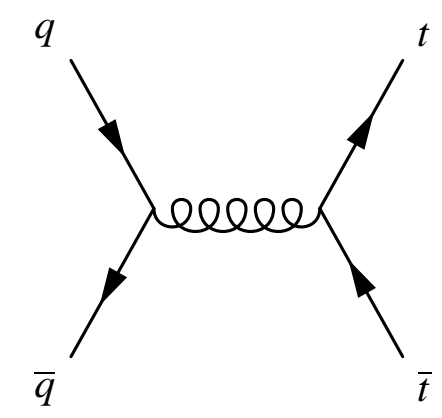

Figure 2.1. Leading order Feynman diagram for $q \bar{q}$ annihilation production of $t \bar{t}$ pairs.

\subsubsection{Parton Level Cross Sections}

The parton level cross sections $\hat{\sigma}_{i j}$ for different processes are expressed as a perturbative series in powers of the strong coupling constant $\alpha_{s}$

$$
\hat{\sigma}_{i j}=C_{0} \alpha_{s}^{2}+C_{1} \alpha_{s}^{3}+C_{2} \alpha_{s}^{4}+\ldots
$$

where $\mathrm{C}_{i}$ are coefficients to be determined from the Feynman diagrams. The leading order (LO) term is proportional to $\alpha_{s}^{2}$, the next-to-leading order (NLO) term to $\alpha_{s}^{3}$ and so on. The production of $t \bar{t}$ pairs at the Tevatron occurs through quark-antiquark $(q \bar{q})$ annihilation and gluon-gluon fusion. The Feynman diagrams for these processes at leading order are shown in Figures 2.1 and 2.2.

The leading order cross sections for these processes can be calculated from the Feynman rules for the above diagrams [12]. The results are

$$
\begin{gathered}
\hat{\sigma}(q \bar{q} \rightarrow t \bar{t})=\frac{2}{9} \frac{4 \pi \alpha_{s}^{2}}{3 \hat{s}}\left(1+\frac{\gamma}{2}\right)(1-\gamma)^{\frac{1}{2}} \\
\hat{\sigma}(g g \rightarrow t \bar{t})=\frac{\pi \alpha_{s}^{2}}{3 \hat{s}}\left[\left(1+\gamma+\frac{1}{16} \gamma^{2}\right) \ln \left(\frac{1+(1-\gamma)^{\frac{1}{2}}}{1-(1-\gamma)^{\frac{1}{2}}}\right)-\left(\frac{7}{4}+\frac{31}{16} \gamma\right)(1-\gamma)^{\frac{1}{2}}\right]
\end{gathered}
$$



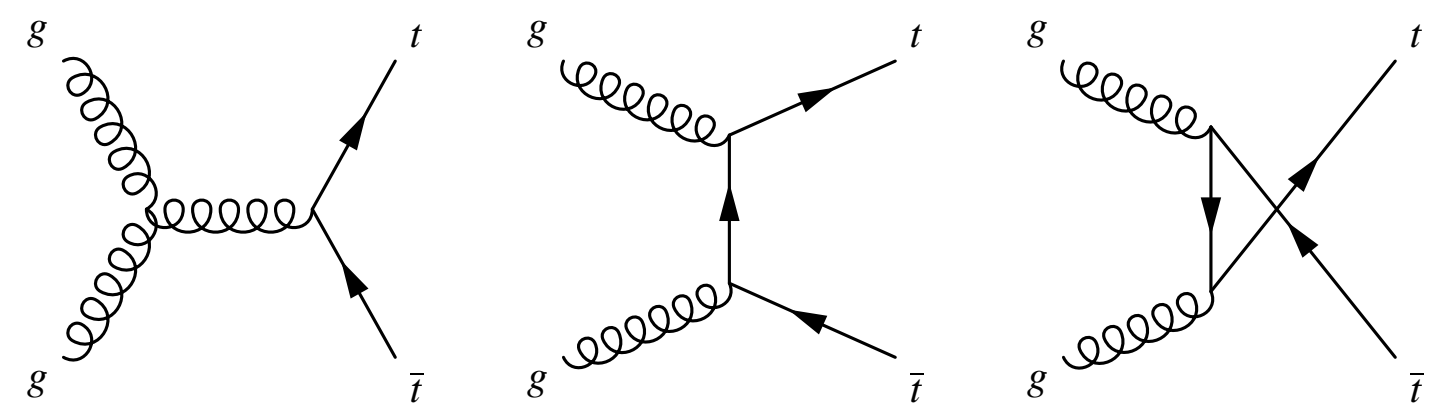

Figure 2.2. Leading order Feynman diagrams for gluon-gluon fusion production of $t \bar{t}$ pairs.

where $\hat{s}$ is the total partonic center of mass energy squared and $\gamma$ is the ratio of the threshold energy squared to $\hat{s}, \frac{4 m_{t}^{2}}{\hat{s}}$. The term $(1-\gamma)^{\frac{1}{2}}$ is the velocity, $\beta$, of the top quark pair. For production near the threshold $\hat{s}=4 m_{t}^{2}, \beta$ is small and $\gamma$ is close to one. The partonic cross sections reduce to

$$
\begin{gathered}
\hat{\sigma}(q \bar{q} \rightarrow t \bar{t}) \approx \frac{4 \pi \alpha_{s}^{2}}{9 \hat{s}} \beta \\
\hat{\sigma}(g g \rightarrow t \bar{t}) \approx \frac{7 \pi \alpha_{s}^{2}}{48 \hat{s}} \beta
\end{gathered}
$$

\subsubsection{Strong Coupling Constant}

The coupling constant for the strong force, $\alpha_{s}$, depends on the momentum scale $\mu$ of the process being described [13]. The relationship between $\alpha_{s}$ and $\mu$ can be written as

$$
\mu \frac{\partial \alpha_{s}}{\partial \mu}=2 \beta\left(\alpha_{s}\right)=-\frac{\beta_{0}}{2 \pi} \alpha_{s}^{2}-\frac{\beta_{1}}{4 \pi^{2}} \alpha_{s}^{3}+\mathcal{O}\left(\alpha_{s}^{4}\right)
$$

where 


$$
\begin{aligned}
& \beta_{0}=11-\frac{2}{3} n_{f} \\
& \beta_{1}=51-\frac{19}{3} n_{f}
\end{aligned}
$$

and $\mathrm{n}_{f}$ is the number of quarks with mass less than $\mu$ (five or six in this case). Solving Equation 2.6 introduces a constant of integration, $\mu_{0}$ [13]. The value of the coupling constant at an arbitrary value of $\mu$ can then be written in terms of this constant as

$$
\ln \left(\frac{\mu^{2}}{\mu_{0}^{2}}\right)=\int_{\alpha_{s}\left(\mu_{0}\right)}^{\alpha_{s}(\mu)} \frac{d \alpha_{s}}{\beta\left(\alpha_{s}\right)}
$$

Substituting Equation 2.6 for $\beta\left(\alpha_{s}\right)$ and ignoring terms of order $\alpha_{s}^{3}$ and higher gives the expression for the running coupling constant

$$
\alpha_{s}(\mu)=\frac{\alpha_{s}\left(\mu_{0}\right)}{1+\frac{\alpha_{s}\left(\mu_{0}\right)}{12 \pi}\left(33-2 n_{f}\right)\left(\ln \left(\frac{\mu^{2}}{\mu_{0}^{2}}\right)\right)}
$$

From Equation 2.9, the value of $\alpha_{s}(\mu)$ increases as the value of $\mu$ decreases, since the term $\left(33-2 \mathrm{n}_{f}\right)$ is positive. It is convenient to introduce a parameter $\Lambda$ that is the value of $\mu_{0}$ at which the coupling constant approaches infinity. We then have

$$
\ln \left(\frac{\mu^{2}}{\Lambda^{2}}\right)=-\int_{\alpha_{s}(\mu)}^{\infty} \frac{d \alpha_{s}}{\beta\left(\alpha_{s}\right)}
$$

which gives

$$
\alpha_{s}\left(\mu^{2}\right)=\frac{12 \pi}{\left(33-2 n_{f}\right) \ln \left(\mu^{2} / \Lambda^{2}\right)}
$$

The parameter $\Lambda$ is referred to as the QCD scale parameter and is determined from experiment to be less than $1 \mathrm{GeV}$.

The fact that the value of the coupling constant decreases as the momentum scale of the process $\mu$ increases is known as asymptotic freedom. For the perturbative approach to be valid, the value of $\alpha_{s}$ should be less than one. The choice of $\mu$ is 
arbitrary, but is usually chosen to be on the order of the mass scale in question, in this case the top quark mass. At this scale, the coupling constant has a value of about 0.1 .

\subsubsection{Parton Distribution Functions}

The Parton Distribution Functions (PDF's) used for Monte Carlo generation and theoretical predictions in this analysis are from the CTEQ collaboration (CTEQ6.1M) [14]. The PDF's are found by combining results from different experiments with nextto-leading order (NLO) QCD calculations. Fits are performed to extract the PDF's from the data and theoretical calculations.

Some of the data that were included in the new calculations include Deep Inelastic Scattering (DIS) results from H1 and ZEUS and the inclusive jet cross section results from $\mathrm{D} \varnothing$. In addition to the new data, an improved scheme for handling correlated errors in the experimental data sets and an improved quantification of the errors on the PDF's was incorporated. The largest difference in the resulting PDF's is a harder gluon spectrum at higher momentum fractions $\mathrm{x}$, driven largely by the jet cross section measurements by $\mathrm{D} \varnothing$ at high pseudo-rapidity.

\subsection{Results}

The production of $t \bar{t}$ pairs at the Tevatron occurs predominately through the processes $q \bar{q} \rightarrow t \bar{t}$ and $\mathrm{gg} \rightarrow t \bar{t}$. At $\sqrt{s}=1.96 \mathrm{TeV}$, the process $q \bar{q} \rightarrow t \bar{t}$ accounts for about $85 \%$ of the total cross section. This is due to the large fraction of the proton momentum needed by the partons for $t \bar{t}$ production, since the threshold for $t \bar{t}$ production is about $17 \%$ of the total center of mass energy. The partons most likely to carry large fractions of the proton momentum are the valence quarks.

Previous calculations at NLO found a significant increase for the cross section over the LO calculation [15]. At a top mass of $175 \mathrm{GeV}$, the $\mathrm{gg} \rightarrow t \bar{t}$ cross section increased 


\begin{tabular}{|c|c|c|c|c|c|c|c|}
\hline \multicolumn{10}{|c|}{$\sigma(\mathrm{pb})$} \\
\hline$\sqrt{s}(\mathrm{TeV})$ & Order & $\mu=\mathrm{m} / 2$ & $\mu=\mathrm{m}$ & $\mu=2 \mathrm{~m}$ & $\mu=\mathrm{m} / 2$ & $\mu=\mathrm{m}$ & $\mu=2 \mathrm{~m}$ \\
\hline & NLO & 5.24 & 5.01 & 4.46 & 5.27 & 5.06 & 4.51 \\
\hline 1.8 & 1PI & 5.40 & 5.52 & 5.36 & 5.43 & 5.58 & 5.43 \\
\hline & PIM & 4.78 & 4.92 & 4.85 & 4.76 & 4.94 & 4.89 \\
\hline \hline & NLO & 6.79 & 6.52 & 5.83 & 6.79 & 6.54 & 5.85 \\
\hline 1.96 & 1PI & 7.00 & 7.17 & 6.99 & 7.01 & 7.21 & 7.04 \\
\hline & PIM & 6.14 & 6.35 & 6.28 & 6.08 & 6.33 & 6.29 \\
\hline
\end{tabular}

TABLE 2.1. Cross section results for $\mathrm{m}_{t}=175 \mathrm{GeV}$. The $1 \mathrm{PI}$ and PIM results are NNLO calculations [11].

by $80 \%$, while the total cross section increased by about $25 \%$. The large increase over the LO calculation is due in a large part to initial state soft gluon emission near the threshold for $t \bar{t}$ production $\left(\hat{s}=4 m_{t}^{2}\right)$.

A technique called resummation is used to calculate the contributions from the soft gluons that take the form of logarithms [16]. A recent calculation of the $t \bar{t}$ production cross section at next-to-next-to-leading order (NNLO) in $\alpha_{s}$ with nextto-next-to-next-to leading logarithm (NNNLL) corrections has been performed by Kidonakis and Vogt [11]. The result for the total $t \bar{t}$ production cross section at $\sqrt{s}$ $=1.96 \mathrm{TeV}$ and $\mathrm{m}_{t}=175 \mathrm{GeV} / \mathrm{c}^{2}$ is

$$
\sigma(p \bar{p} \rightarrow t \bar{t})=6.77 \pm 0.42 p b
$$

with the results for different choices of $\mu$, different kinematics, and two different choices for the PDF's shown in Figure 2.3 and summarized in Table 2.1.

The uncertainty is dominated by the choice of kinematics (single particle inclusive, 1PI, vs. pair invariant mass, PIM).

$$
\begin{array}{lll}
q\left(p_{a}\right)+\bar{q}\left(p_{b}\right) & \left.\rightarrow t\left(p_{1}\right)+X \bar{t}\right]\left(p_{2}\right) & (1 P I) \\
q\left(p_{a}\right)+\bar{q}\left(p_{b}\right) & \rightarrow t \bar{t}(p)+X(k) & (P I M)
\end{array}
$$




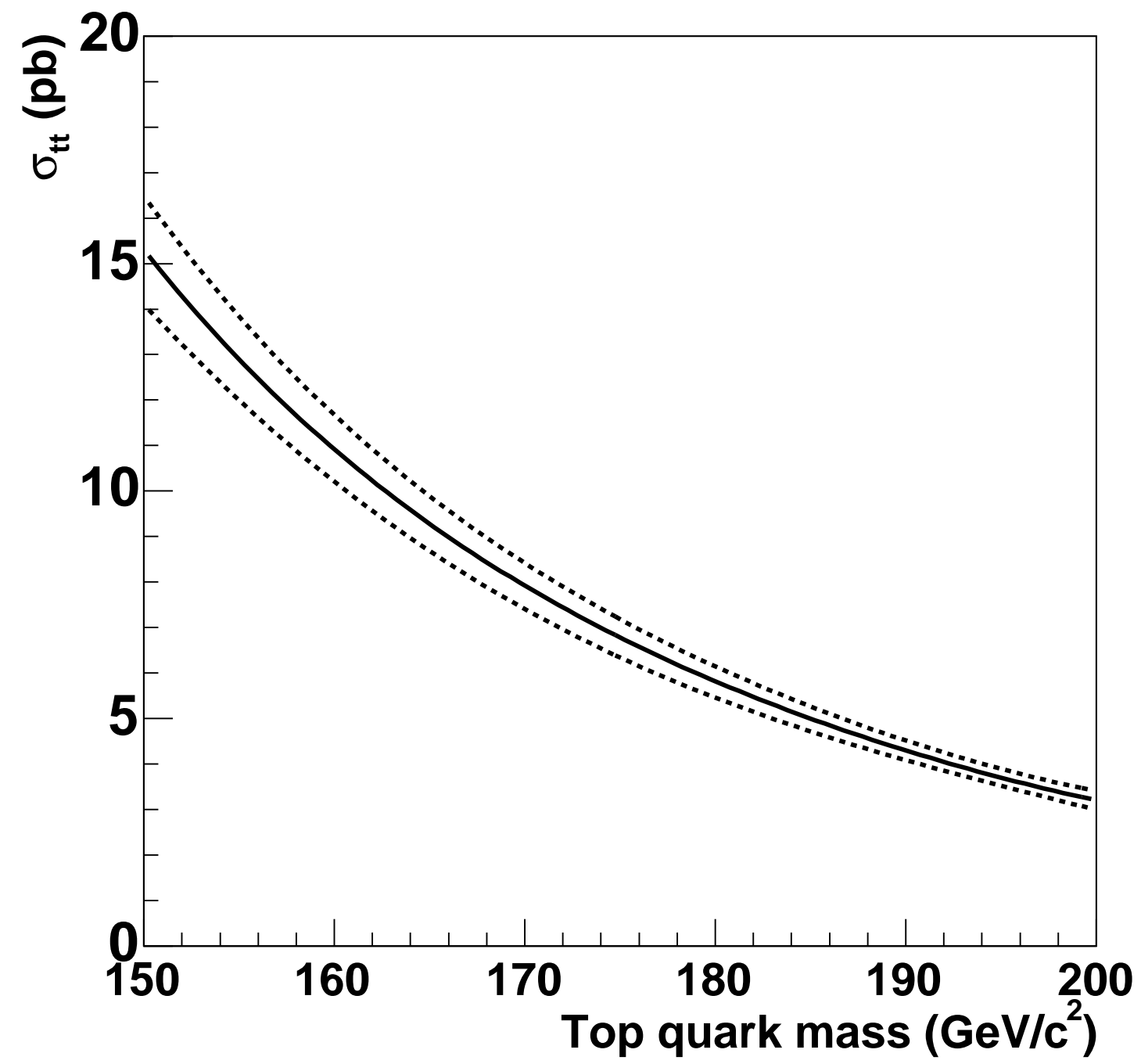

Figure 2.3. The theoretical cross section for $t \bar{t}$ production at $\sqrt{s}=1.96 \mathrm{TeV}$ as a funtion of the top quark mass. The average NNLO result is the solid line, while the dashed lines are the 1PI (upper) and PIM (lower) results. 
The cross section should not depend on the choice of kinematics and the calculated difference is an indication that corrections beyond NNNLL have an impact on the cross section. However, this difference is lower than previous calculations with NNLL corrections, especially near the production threshold.

Variations of the cross section for different choices of the renormalization scale factor $\mu$ are an indication of the importance of the ignored terms in the perturbative expansion. Table 2.1 shows that the NNLO results (1PI and PIM) have small variations for different values of $\mu$, indicating that the ignored higher order terms $\left(\alpha_{s}^{5}\right.$ and above) do not contribute much to the cross section.

\subsection{Top Decay}

In the Standard Model, the top quark decays via the weak interaction. The top decays $\geq 99.8 \%$ of the time to a real $\mathrm{W}$ boson and a bottom quark, assuming three generations [13]. The Feynman diagram for the decay of the top quark is given in

Figure 2.4. The top quark decay width can be written as (neglecting terms of order $m_{b}^{2} / m_{t}^{2}, \alpha_{s}^{2}$, and $\left.\left(\alpha_{s} / \pi\right) m_{W}^{2} / m_{t}^{2}\right)$

$$
\Gamma(t \rightarrow b W)=\frac{G_{F} m_{t}^{3}}{8 \pi \sqrt{2}}\left(1-\frac{m_{W}^{2}}{m_{t}^{2}}\right)^{2}\left(1+2 \frac{m_{W}^{2}}{m_{t}^{2}}\right)\left[1-\frac{2 \alpha_{s}}{3 \pi}\left(\frac{2 \pi^{2}}{3}-\frac{5}{2}\right)\right]
$$

For a top mass of $180 \mathrm{GeV} / \mathrm{c}^{2}$, the decay width is $1.56 \mathrm{GeV} / \mathrm{c}^{2}$ [17]. The lifetime is the inverse of the decay width

$$
\begin{aligned}
\tau & =\frac{\hbar}{\Gamma} \\
& =4.2 \times 10^{-25} \mathrm{sec} .
\end{aligned}
$$

The lifetime is about a factor of ten times shorter than the typical hadronization timescale and thus the top quark decays before hadronization. 


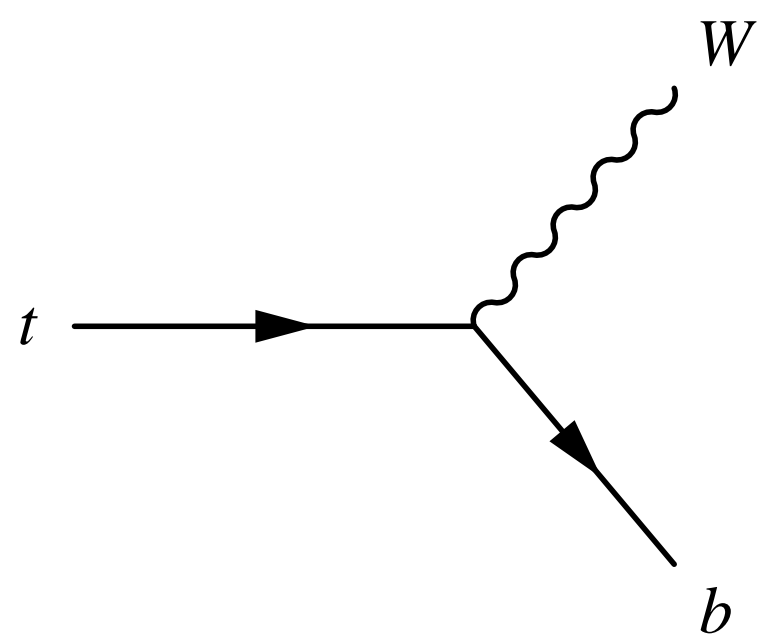

Figure 2.4. The Feynman diagram for the weak decay of the top quark.

\begin{tabular}{|c|c|c|c|c|}
\hline & $\begin{array}{c}W b \rightarrow e \nu_{e} j \\
(1 / 9)\end{array}$ & $\begin{array}{c}W b \rightarrow \mu \nu_{\mu} j \\
(1 / 9)\end{array}$ & $\begin{array}{c}W b \rightarrow \tau \nu_{\tau} j \\
(1 / 9)\end{array}$ & $\begin{array}{c}W b \rightarrow j j j \\
(2 / 3)\end{array}$ \\
\hline$W b \rightarrow e \nu_{e} j(1 / 9)$ & $1 / 81$ & $1 / 81$ & $1 / 81$ & $2 / 27$ \\
\hline$W b \rightarrow \mu \nu_{\mu} j(1 / 9)$ & $1 / 81$ & $1 / 81$ & $1 / 81$ & $2 / 27$ \\
\hline$W b \rightarrow \tau \nu_{\tau} j(1 / 9)$ & $1 / 81$ & $1 / 81$ & $1 / 81$ & $2 / 27$ \\
\hline$W b \rightarrow j j j(2 / 3)$ & $2 / 27$ & $2 / 27$ & $2 / 27$ & $4 / 27$ \\
\hline
\end{tabular}

TABLE 2.2. The final states for $t \bar{t}$ decay, classified by the decay of the $\mathrm{W}$ bosons. The bottom quarks from the decay of the top quark and the light quarks from the decay of the $\mathrm{W}$ boson appear as jets in the detector and are denoted as $j$ in the final state. The dimuon channel has a branching ration of $1 / 81$.

We classify the decays of the $t \bar{t}$ pairs based on the decay of the $\mathrm{W}$ bosons. The $\mathrm{W}^{+}$decays to a $q \bar{q}$ pair $(u \bar{d}, c \bar{s}) 2 / 3$ of the time and to a lepton-neutrino pair $\left(e^{+} \nu_{e}, \mu^{+} \nu_{\mu}, \tau^{+} \nu_{\tau}\right) 1 / 3$ of the time. The difference between the quark and lepton decay branching fractions is due to the three colors of quarks available for the decay. The branching fractions for the various $t \bar{t}$ decays are shown in Table 2.2. The bottom quarks from the decay of the top quark and the light quarks from the decay of the W boson appear as jets in the detector.

This analysis looks for dimuon events from $t \bar{t}$ production. The expected signature in the detector is two high $\mathrm{P}_{\mathrm{T}}$ muons of opposite sign from the decay of the $\mathrm{W}$ bosons 
and two energetic jets from the bottom quarks. Finally, the two neutrinos from the decay of the $\mathrm{W}$ bosons should leave missing transverse energy in the detector because the neutrinos carry off energy without interacting in the detector. 


\section{Chapter 3}

\section{EXPERIMENTAL APPARATUS}

The Fermilab Tevatron is a proton-antiproton $(p \bar{p})$ synchrotron collider with a total center of mass energy of $\sqrt{s}=1.96 \mathrm{TeV}$. The Tevatron uses superconducting magnets to steer and focus the beams around the 1000 meter radius ring. Collisions are initiated at two regions in the Tevatron, called $\mathrm{B} \varnothing$ and $\mathrm{D} \varnothing$. The $\mathrm{D} \varnothing$ detector is a large, multi-purpose detector used to record the results of these collisions. Roughly one million individual detector channels are used to identify photons, electrons, jets, and muons that result from the collisions and reconstruct the underlying event.

\subsection{Fermilab Tevatron}

The Tevatron is actually the last in a chain of five accelerators used to produce protons and accelerate them to $980 \mathrm{GeV}$ (Figure 3.1). In addition, a special antiproton debuncher and accumulator ring is used to collect, cool, and store antiprotons produced by colliding protons with a nickel target. A detailed overview of the accelerators can be found in [18].

All protons begin in a negative hydrogen ion source that produces $18 \mathrm{keV} \mathrm{H}^{-}$ ions. The $\mathrm{H}^{-}$ions are accelerated to $750 \mathrm{keV}$ by a Cockroft-Walton electrostatic accelerator and then sent to a 146 meter, two-stage linear accelerator (Linac) that increases the energy to $400 \mathrm{MeV}$. The third accelerator in the chain is the Booster, a $75 \mathrm{~m}$ radius synchrotron that increases the energy to $8 \mathrm{GeV}$. At the transfer from the Linac to the Booster, a carbon foil strips the two electrons from the $\mathrm{H}^{-}$ion, leaving the proton. As the Booster accelerates the protons, they are grouped into individual RF buckets around the Booster. 


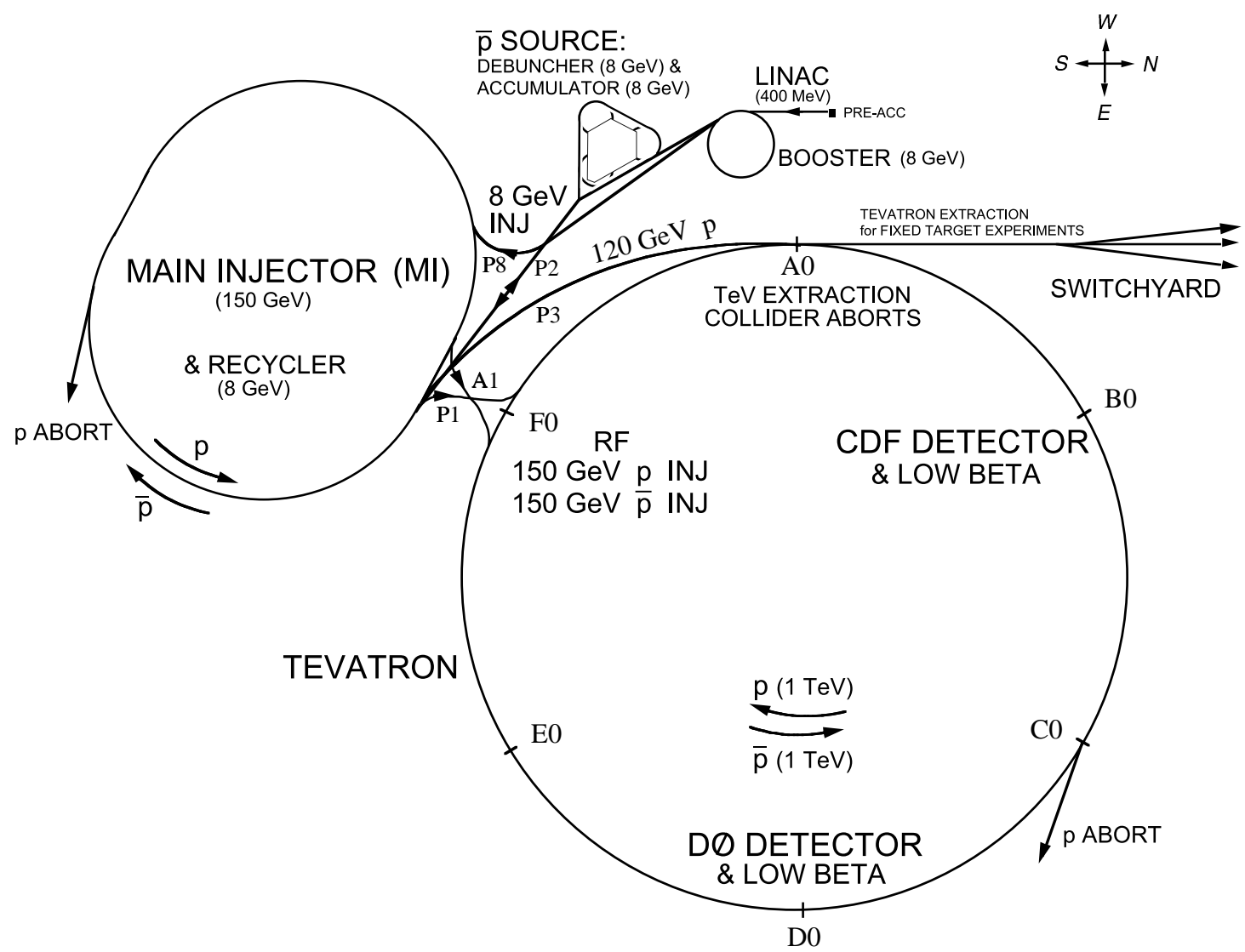

Figure 3.1. The Fermilab accelerator complex. 
From the Booster, the protons enter the Main Injector [19]. This is a new accelerator at Fermilab that replaces the old Main Ring. The Main Injector is used in two ways. During the accumulation of antiprotons, referred to as stacking, the Main Injector accelerates protons to $120 \mathrm{GeV}$ and sends them to a nickel target to produce antiprotons. The antiprotons are collected and stochastically cooled in the Debuncher ring at an energy of $8 \mathrm{GeV}$. Stochastic cooling refers to reducing the differences in the longitudinal and transverse momenta of the antiprotons by providing corrective 'kicks' to the beam. Cooled antiprotons are then sent to the Accumulator ring, where they are stored.

When enough antiprotons have been collected (typically around $150 \times 10^{10}$ ), the accumulation stops. At this point, protons from the Booster are transfered to the Main Injector, where they are accelerated to $150 \mathrm{GeV}$. To increase the number of protons that are sent to the Tevatron, the individual proton bunches from the Booster are coalesced into one large bunch in the Main Injector and transfered to the Tevatron. This process is repeated until 36 bunches of protons have been sent to the Tevatron. At this point, antiprotons from the accumulator are sent to the Main Injector, accelerated to $150 \mathrm{GeV}$, and sent to the Tevatron.

The Tevatron is a $1000 \mathrm{~m}$ radius synchrotron that accelerates protons and antiprotons from $150 \mathrm{GeV}$ to $980 \mathrm{GeV}$. Unlike the Booster and Main Injector, the dipole bending magnets and quadrapole focusing magnets are made of a superconducting niobium/titanium alloy that is kept at a temperature of $4 \mathrm{~K}$. The superconducting magnets allow higher currents than conventional magnets, and thus stronger magnetic fields that can bend and focus higher energy beams. The Tevatron produces collisions at the two interaction regions by using special focusing magnets called the low-beta quadrapole magnets. These magnets reduce the diameter of the beams to about $40 \mu \mathrm{m}$, inducing collisions between the protons and antiprotons.

The resonant frequency of the Tevatron Radio Frequency (RF) cavities is 53.1 $\mathrm{MHz}$, which corresponds to an RF clock period of 18.8 ns. A total of $1113 \mathrm{RF}$ bunch 
cavities exist in the Tevatron. The current scheme for colliding beam operation at the Tevatron is to fill 36 of the RF buckets with protons and 36 with antiprotons. The bunch cavities are filled so the protons and antiprotons interact at the two collision regions every $396 \mathrm{~ns}$ ( 21 x $18.8 \mathrm{~ns}$ ). The 36 bunches of protons and antiprotons are divided into three 'superbunches' of twelve and are separated by gaps of approximately $2.2 \mu \mathrm{s}$.

A final accelerator, the Recycler, sits directly below the Main Injector. The Recycler can accept antiprotons from the Accumulator during stacking operations and store them until they are needed. The Recycler helps Tevatron performance in two ways. First, as the number of antiprotons increases in the Accumulator, the production, or stacking, rate for new antiprotons decreases. By periodically transferring the accumulated antiprotons to the Recycler, the stacking rate stays high. As of Fall 2004, antiprotons have been successfully transfered to the Recycler, stored, and later injected into the Tevatron for collisions. The second advantage of the Recycler is the ability to further cool the antiprotons using electron cooling. Electron cooling uses electron beams traveling parallel to the antiprotons to reduce the size of the antiproton bunches. Reducing the size of the antiproton bunches that are injected into the Tevatron gives more luminosity per antiproton bunch. The use of electron cooling is expected to begin in late 2005 .

\subsection{DØ Detector}

The D $\varnothing$ experiment (Figure 3.2) uses several independent detectors to fully record the results of $p \bar{p}$ collisions at the Fermilab Tevatron [20] [21]. Closest to the interaction point, an inner tracking detector, comprised of a silicon tracker and a scintillating fiber tracker, is enclosed in a 2 Tesla solenoidal field. Together, the tracking detectors reconstruct the location of the event vertices and provide momentum measurements for charged particles by measuring the curvature of the tracks in the magnetic field. 


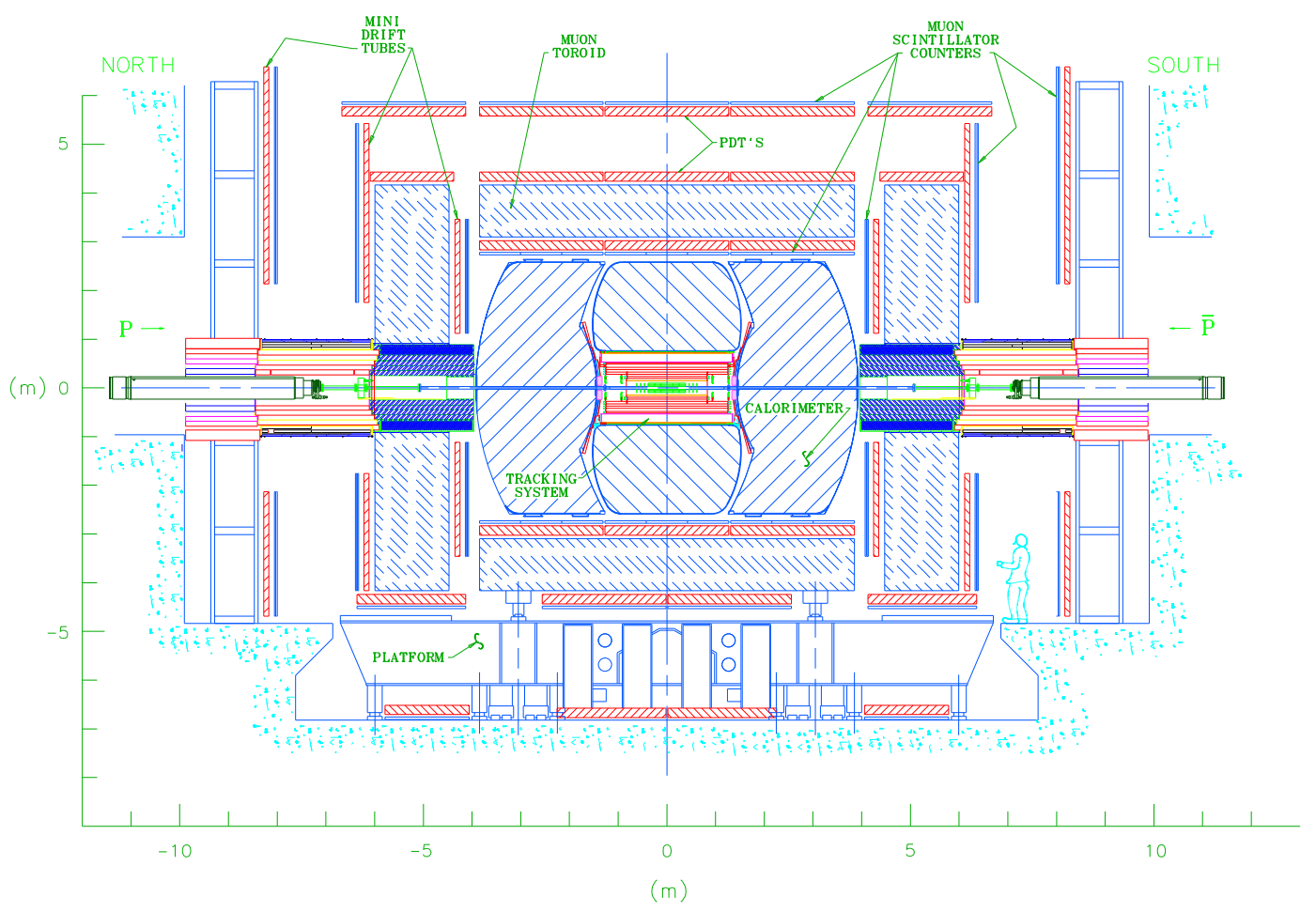

Figure 3.2. The D $\varnothing$ detector.

Outside of the solenoid is a liquid argon sampling calorimeter that measures the energy of photons, electrons, and hadrons. Muon detectors are located outside of the calorimeter to detect and track muons produced in the event. Three layers of wire chambers, with a 1.8 Tesla toroid between the first and second layers, provide position and momentum measurement for the muons.

\subsubsection{Silicon Microstrip Tracker}

The inner-most part of the D $\varnothing$ detector is the Silicon Microstrip Tracker (SMT). The SMT is composed of roughly 800,000 channels of silicon strips with a typical distance between adjacent strips of $50 \mu \mathrm{m}$. The excellent granularity is used to precisely measure the primary event vertex, detect secondary vertices that can be used to tag events with heavy quarks, and to improve the momentum resolution of charged particle tracks found with the Central Fiber Tracker. 


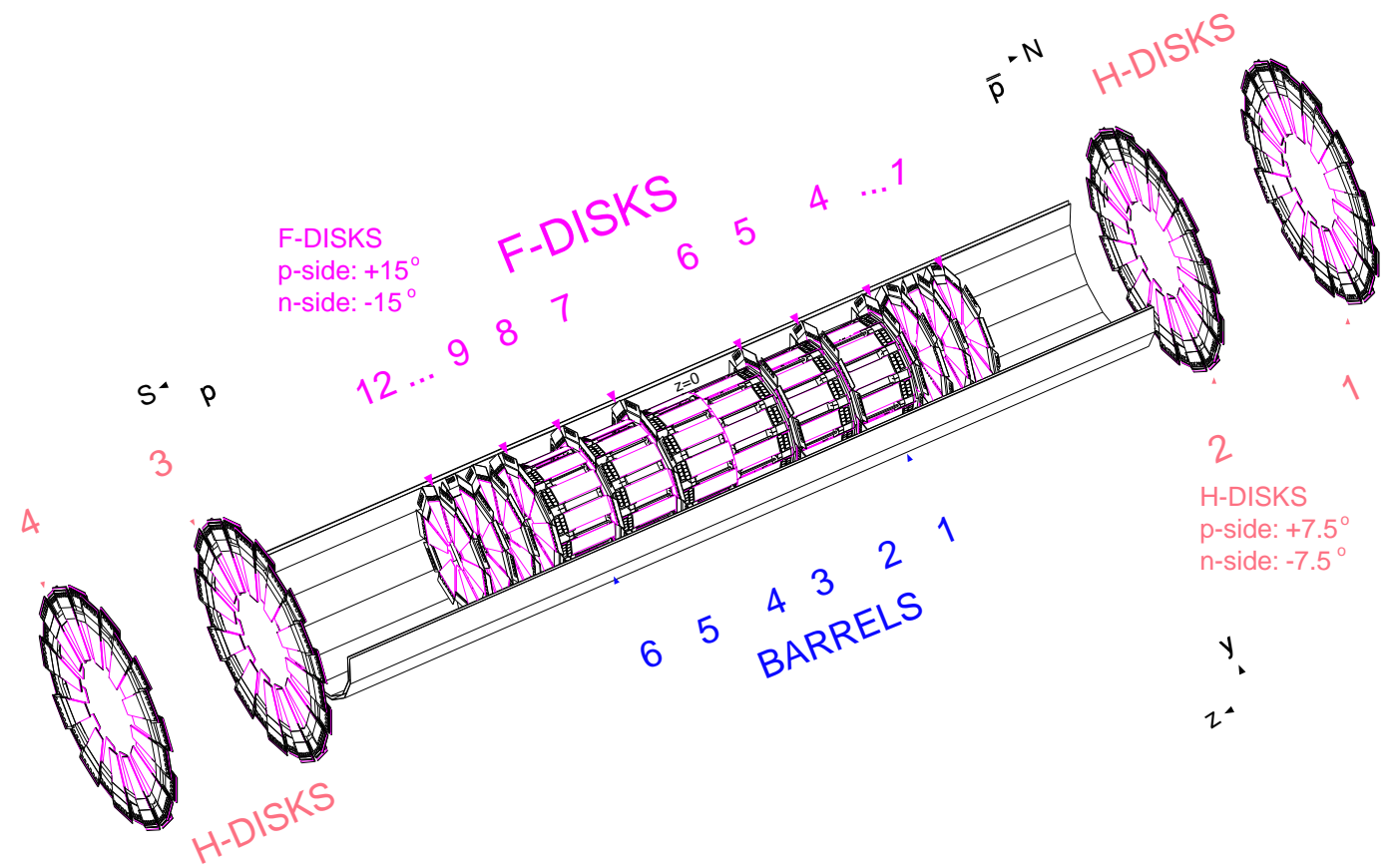

Figure 3.3. The Silicon Microstrip Tracker (SMT). A combination of barrel and disk detectors allow 3D track reconstruction to $|\eta|<3.0$.

The SMT design is a hybrid of barrel and disk detectors and is shown in Figure 3.3. The barrel and central disk (F-disk) detectors are used to detect tracks at a large angle with respect to the beam $(|\eta|<1.5)$, while the forward disk (H-disk) detectors provide coverage to $|\eta|<3.0$. Table 3.1 summarizes the silicon design parameters for the barrel and disk detectors [22].

The barrel detector is arranged in four layers in radius $\rho$. Layers two and four are double-sided (DS) silicon with a stereo angle of $2^{\circ}$. The four barrels closest to $Z=0$ are DS silicon with a $90^{\circ}$ stereo angle for layers one and three, while the outer two barrels are single-sided (SS) silicon for layers one and three. The four layers of the barrel detector have radii at $2.7,4.5,6.6$, and $9.4 \mathrm{~cm}$.

The F-Disk assemblies are made of double-sided silicon with $\pm 15^{\circ}$ stereo strips on the p- and n-side. The inner radius of the F-Disk assemblies is $2.6 \mathrm{~cm}$, with an outer radius of $10 \mathrm{~cm}$. The inner six F-disks are mounted on the ends of the six barrel 


\begin{tabular}{|l|l|l|l|}
\hline Detector & Silicon Type & Stereo Angle & Radius (cm) \\
\hline Barrel & & \\
Layer 1 inner barrels & Single-sided & & 2.7 \\
Layer 1 outer barrels & Double-sided & $90^{\circ}$ & 2.7 \\
Layer 2 & Double-sided & $2^{\circ}$ & 4.5 \\
Layer 3 inner barrels & Single-sided & & 6.6 \\
Layer 3 outer barrels & Double-sided & $90^{\circ}$ & 6.6 \\
Layer 4 & Double-sided & $2^{\circ}$ & 9.4 \\
\hline Disk & & & \\
F-Wedges & Double-sided & $\pm 15^{\circ}$ & $2.6-10$ \\
H-Wedges & Single-sided & $\pm 7.5^{\circ}$ & $9.6-23.6$ \\
\hline
\end{tabular}

TABLE 3.1. Silicon Microstrip Tracker design parameters.

assemblies, while the outer six are mounted in groups of three end disk modules on each side (Figure 3.3). In the forward-most region, the H-disks are composed of backto-back single-sided silicon chips with effective $\pm 7.5^{\circ}$ stereo strips. These have an inner radius of $9.6 \mathrm{~cm}$ and an outer radius of $23.6 \mathrm{~cm}$.

Readout of the silicon detector is accomplished using the SVX-IIe custom integrated circuit that is mounted directly on the ladder and wedge structures [23]. Each SVX chip stores the collected charge for 128 channels in a 32-deep analog pipeline. When an event passes the Level 1 trigger, the collected charge for that event is digitized and channels above a given threshold are readout.

\subsubsection{Central Fiber Tracker}

The Central Fiber Tracker (CFT) is also enclosed within the 2 T magnetic field [24]. The CFT is comprised of eight layers of scintillating fibers $835 \mu \mathrm{m}$ thick and either $1.66 \mathrm{~m}$ (inner two layers) or $2.52 \mathrm{~m}$ long. The innermost layer is located just outside the silicon barrel assembly at a radius of $20 \mathrm{~cm}$, while the outer layer is located at a radius of $51.5 \mathrm{~cm}$. Each layer is comprised of fiber doublets mounted axially (along the Z-axis) and doublets mounted with a $2^{\circ}$ stereo component (Figure 3.4) to measure 


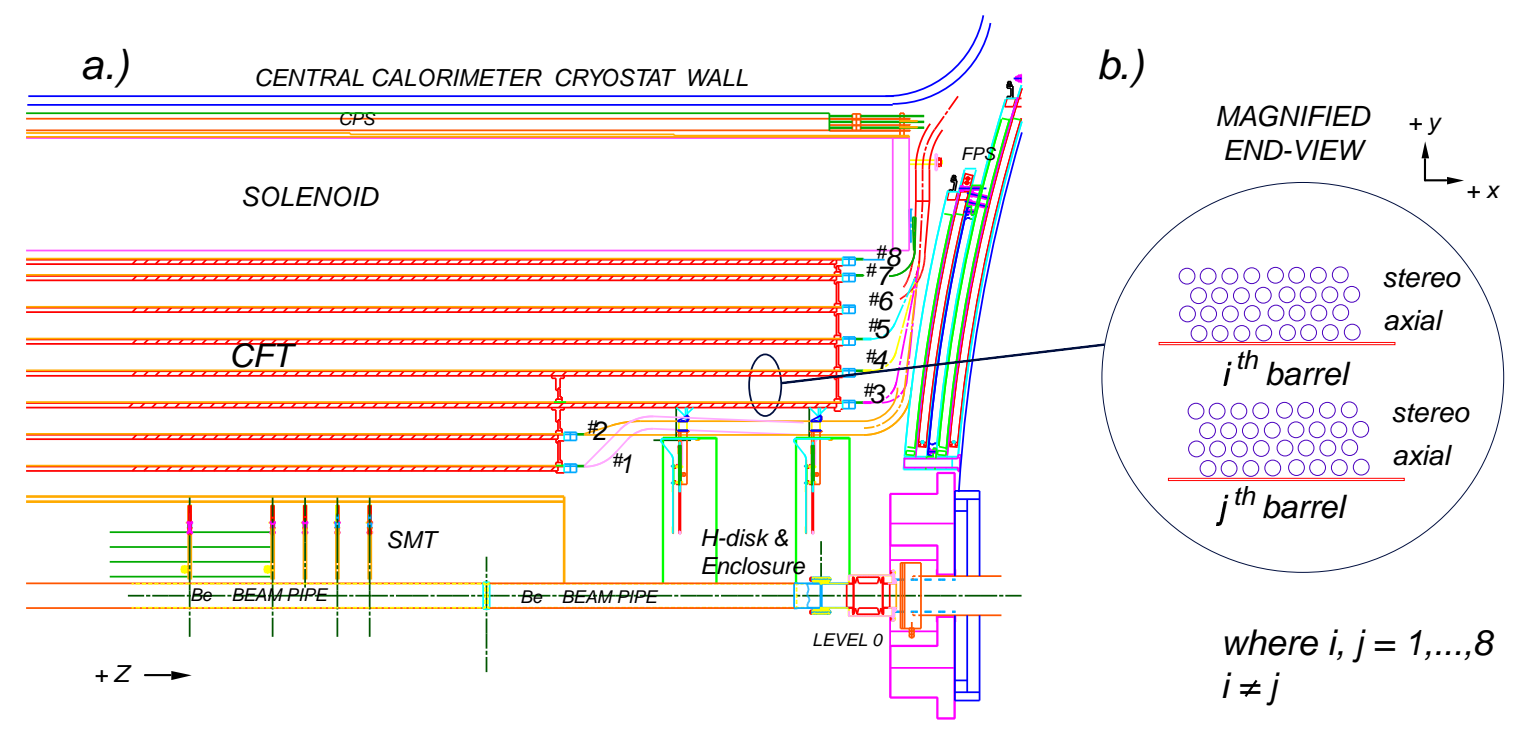

Figure 3.4. The CFT detector.

charged particles in both the $r-\phi$ and $r-z$ plane.

Charged particles passing through a scintillating fiber cause the emission of light at a wavelength of $340 \mathrm{~nm}$. A wavelength shifter is introduced to re-emit light at $\lambda=530 \mathrm{~nm}$. The photons are reflected down the fiber and into waveguides that carry the light to the Visible Light Photon Counters (VLPC's). The VLPC's are solid state photomultipliers that operate at a temperature of roughly $4 \mathrm{~K}$. About 1000 photons are produced when a charged particle passes through a fiber, while the total transfer efficiency from the fiber to the VLPC is about 1.5\%. Thus, roughly 10-20 photons from a given fiber make it into the VLPC. The output of the VLPC is stored in SVX-IIe chips for digitization and readout. In addition, the VLPC signals are discriminated and sent to the Level 1 Central Track Trigger (L1CTT).

The CFT provides transverse momentum measurements for charged particle tracks by determining the curvature of the track in the magnetic field. The $\mathrm{P}_{\mathrm{T}}$ resolution for tracks reconstructed with the CFT only at $\eta=0$ can be parameterized by

$$
\frac{\sigma_{P_{T}}}{P_{T}}=\sqrt{0.015^{2}+\left(0.0014 \cdot P_{T}\right)^{2}}
$$


where $P_{T}$ is in units of $\mathrm{GeV} / \mathrm{c}$ [24]. For a track with momentum of $20 \mathrm{GeV} / \mathrm{c}$, this gives a $\frac{\sigma_{P_{T}}}{P_{T}}$ of about $3 \%$.

\subsubsection{Preshower Detectors}

Preshower detectors have been installed between the solenoid and the calorimeter for Run II. The preshower detectors are used to improve electron identification and energy resolution. Lead absorber plates are used to induce an electromagnetic shower for electrons and photons, which are then detected by scintillating fibers. Backgrounds, such as charged pions, will tend to deposit only minimum ionizing energy and can be separated from electrons and photons.

The central preshower detector consists of one layer of lead followed by three layers of scintillating fibers (one axial, two stereo) [25]. Electrons will have a matching track in the central tracker, while photons will not. In the forward region, where the central tracker coverage ends, the lead absorber is sandwiched between two layers of scintillating fibers to distinguish electrons from photons [26].

\subsubsection{Calorimetry}

The $\mathrm{D} \varnothing$ calorimeter surrounds the tracking detectors and the solenoid and is used to measure the total energy of all charged and neutral particles except muons and neutrinos. To accomplish this, the calorimeter uses a dense absorber material (depleted uranium, copper, or stainless steel) to induce electromagnetic or hadronic particle showers. Between layers of absorbers are regions of liquid argon (LAr) that sample the ionizing particles that pass through. The charge collected from this ionization is proportional to total number of particles produced in the shower, and thus the energy of the incident particle. The calorimeter is largely unchanged from Run I and details can be found in [20]. Details of the electronics upgrades for Run II are in [27].

One unit cell in the calorimeter is comprised of a layer of absorber, a LAr gap, 


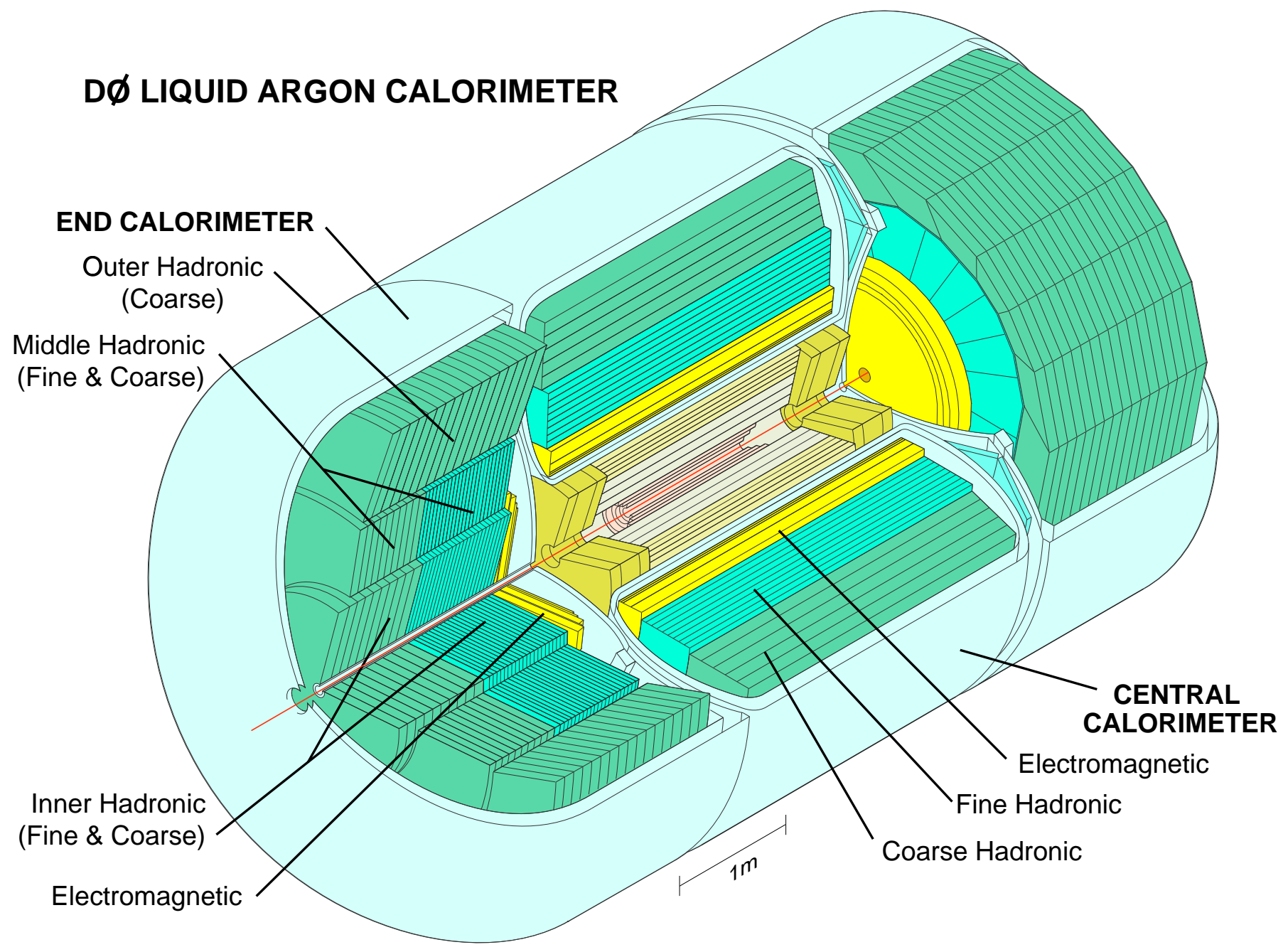

Figure 3.5. The D $\varnothing$ calorimeter. 
and a signal board. The signal board is a copper sheet sandwiched between two 0.5 mm sheets of G10. The G10 has a resistive coating on the side facing the LAr and is held to a high positive potential. Electrons produced by the passage of ionizing particles through the LAr travel to the signal board, inducing a mirror charge on the copper sheet. Several layers of signal boards are chained together and the collected charge is readout.

The DØ calorimeter (Figure 3.5) is divided into a central cryostat (CC) and two end cryostats (EC). Each cryostat has electromagnetic (EM), fine hadronic (FH), and coarse hadronic $(\mathrm{CH})$ modules, which are alternating layers of absorber and LAr cells. The cells are arranged in pseudo-projective towers that point to the interaction region. The typical tower size in $\eta-\phi$ is $0.1 \times 0.1$, except in the third layer of the EM modules, where the size is $0.05 \times .05$, and in the region of $|\eta|>3.2$, where the cells are larger.

In the central cryostat, the 32 electromagnetic modules use $3 \mathrm{~mm}$ thick uranium absorber plates with a thickness of $21 X_{0}$. The readout of the central EM modules is divided into four layers, with the maximum energy deposition occurring in the finely segmented third layer. The sixteen fine hadronic modules surround the EM portion and use $6 \mathrm{~mm}$ thick uranium absorbers and three readout layers. Finally, the sixteen coarse hadronic modules use $46.5 \mathrm{~mm}$ copper absorbers and are arranged in one readout layer. The total thickness in nuclear interaction lengths of the fine and coarse hadronic modules is about $7 \lambda$. The central cryostat provides coverage to $|\eta|$ $<1$.

The end cryostats have one EM module with $4 \mathrm{~mm}$ thick uranium absorber plates and has a thickness of $20 X_{0}$. Behind the EM module are three concentric cylinders of hadronic modules, labeled inner, middle, and outer hadronic. The inner and middle hadronic modules have both fine hadronic and coarse hadronic portions, while the outer hadronic modules are only coarse. The absorber material in the coarse hadronic modules is stainless steel. The eta coverage for the end cryostats is roughly $1.3<|\eta|$ 
$<4.0$.

The resolution of the calorimeter is parameterized by

$$
\left(\frac{\sigma}{E}\right)^{2}=C^{2}+\frac{S^{2}}{E}+\frac{N^{2}}{E^{2}}
$$

where $\mathrm{C}$ is a constant terms that represents calibration errors, $\mathrm{S}$ is the sampling fluctuations, and $\mathrm{N}$ is the noise contributions [20]. These parameters were measured using electron and pion test beams. For electrons,

$$
C=0.003 \pm 0.002, S=0.157 \pm 0.005(G e V)^{\frac{1}{2}}, N \approx 0.140 \mathrm{GeV}
$$

while for pions

$$
C=0.032 \pm 0.004, S=0.41 \pm 0.04(\mathrm{GeV})^{\frac{1}{2}}, N \approx 1.28 \mathrm{GeV}
$$

\subsubsection{Muon System}

Detection of muons is performed by wire chambers and scintillation counters located outside the calorimeter. Unlike the lighter electrons, muons deposit only minimum ionizing energy as they traverse the calorimeter, losing on average about $1.5 \mathrm{GeV}$. To measure the muon position, three layers of wire chambers surround the calorimeter. A $1.8 \mathrm{~T}$ iron toroid is located between the first and second layers, which allows a momentum measurement via the bend angle of the track. Tracks found in the muon system can then be matched to tracks found in the central tracker.

The D $\varnothing$ muon system is divided into two regions - a central and forward system. The central region covers the region $|\eta|<1.0$, while the forward system provides coverage for $1.0<|\eta|<2.0$. The three layers in each region are labeled A, B, and C, with the A-layer closest to the interaction point. 


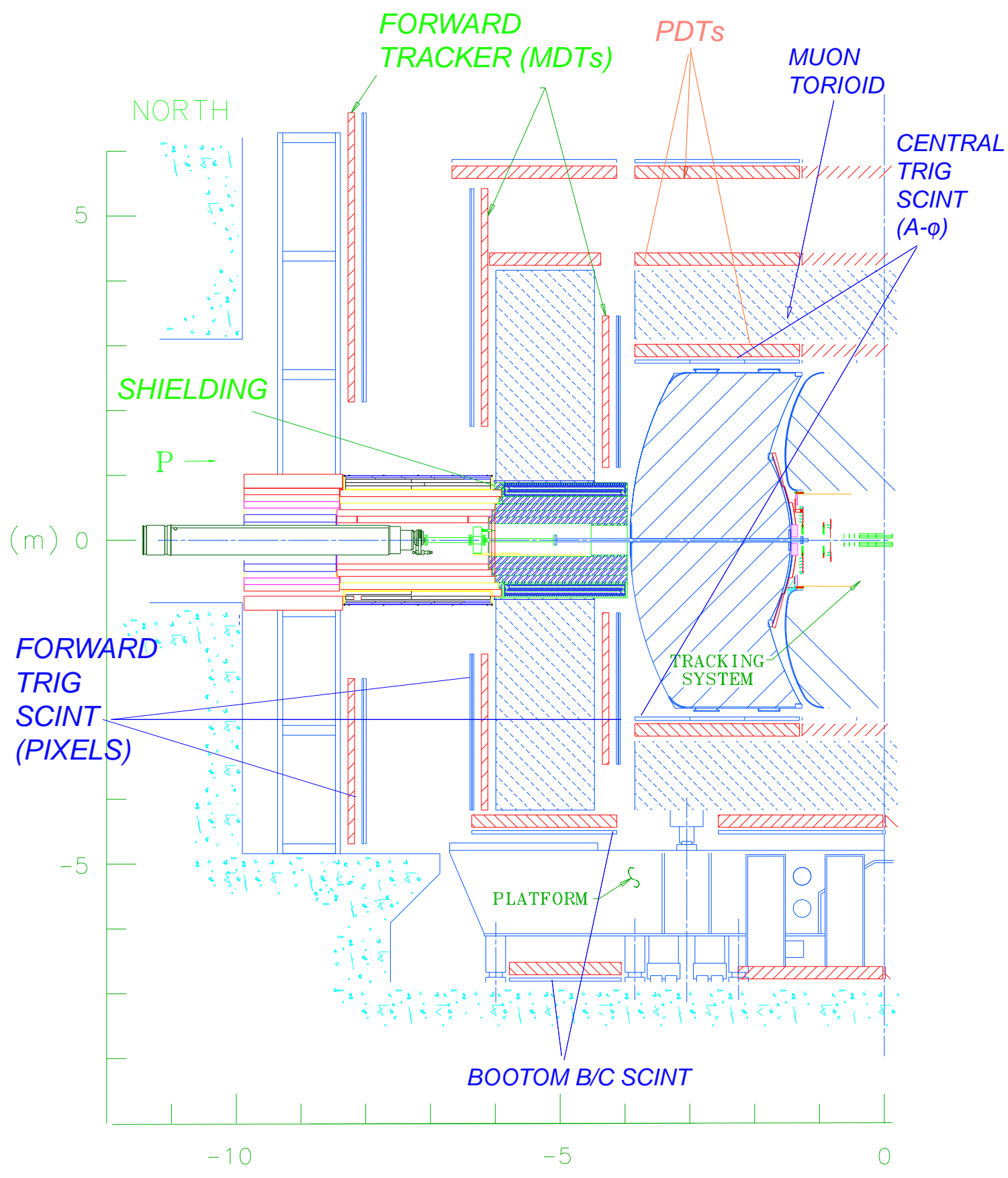

(m)

Figure 3.6. The muon detector. 


\section{Tracking Chambers - Central}

The central region uses three layers of Proportional Drift Tubes (PDT's) to measure the location of muons. These are the original chambers from Run I with new cathode pads and readout electronics. A total of 94 chambers give three layers of coverage in the top $75 \%$ of the detector and two layers in the bottom, where mechanical supports for the detector restrict placement. Each chamber is comprised of 10.1 x $5.5 \mathrm{~cm}$ cells set into three or four decks [28].

The PDT's are oriented to give maximum resolution in the $\eta$ coordinate, which is bend direction of the toroid. While measurement in the $\phi$ direction is possible using readout of the cathode pads, this has been supplemented using the scintillation counters in the A-layer and 3D central tracks matched to muon system tracks.

To reduce the number of bunch crossings that the drift time spans, a fast gas is needed for the PDT's. The operating gas is argon (80\%), methane (10\%), and CF4 (10\%). This gives a maximum drift time of about 550ns, which is about four $132 \mathrm{~ns}$ bunch crossings.

\section{Tracking Chambers - Forward}

The forward system uses three layers of Mini Drift Tubes (MDT's) in both the north and south region to cover the region $1.0<|\eta|<2.0$. Like the PDT's, the MDT's are arranged in either four deck (A-layer) or three deck (B- and C-layer) configurations. The MDT cell size is $1 \mathrm{~cm}^{2}$, and the operating gas is $\mathrm{CF}_{4}(90 \%)-\mathrm{CH}_{4}$ $(10 \%)[29]$.

As with the PDT's, the orientation of the MDT wires is roughly perpendicular to the bend angle of the muons through the toroid. The maximum drift time is about $60 \mathrm{~ns}$, and the position resolution is about $720 \mu \mathrm{m}$, which is dominated by the $18.8 \mathrm{~ns}$ digitizing resolution of the electronics. Scintillation counters with a $4.5^{\circ} \phi$ segmenta- 
tion are used in all three layers to provide a position measurement along the wire axis.

\section{Trigger Scintillation Counters - Central}

In addition to the wire drift chambers, the central region has scintillation counters to help in triggering and provide measurement in the $\phi$ direction. A-layer coverage is provided by the A- $\phi$ counters. These counters have $4.5^{\circ}$ segmentation in $\phi$ and are $33 \frac{1}{4}$ inches in the $\mathrm{Z}$ direction, designed to match the size of the PDT's. The A- $\phi$ counters have a timing resolution of about 2.5 ns offline and a Level 1 trigger gate width of $24 \mathrm{~ns}$. This allows the rejection of out-of-time backgrounds both in the online trigger and in the offline reconstruction. The scintillator hits also act to confirm the bunch crossing for Level 1 hits from the PDT's, since the drift time is longer than the time between bunch crossings.

In addition to the new A- $\phi$ counters, the central region also has the Cosmic Cap scintillators from Run I on the outer (C) layer. These counters are 25 in wide and range from 81.5 to 113 in long. The counters are oriented to give maximum resolution in the $\eta$ coordinate. Additional scintillation counters have been placed on the bottom B- and C-layers to aid in triggering. Details of all of the central scintillation counters can be found in [28].

\section{Trigger Scintillation Counters - Forward}

The scintillation counters in the forward region provide full coverage for all three layers. The forward counters are called the pixel scintillation counters and have $4.5^{\circ}$ segmentation in $\phi$ and roughly 0.1 segmentation in $\eta$. They are arranged in a projective fashion from the nominal interaction point. The uniform segmentation in $\eta$ and $\phi$ means the individual counters come in a variety of sizes, from $17 \times 24 \mathrm{~cm}^{2}$ to $60 \times 106 \mathrm{~cm}^{2}$ [30]. The offline time resolution for the pixel scintillation counters 
for reconstructed muons is about $2 \mathrm{~ns}$, while Level 1 trigger gate width is $30 \mathrm{~ns}$ [31].

\subsection{DØ Trigger System}

The DØ detector uses a three-level trigger system to select interesting events online and reduce the $1.7 \mathrm{MHz}$ interaction rate down to the $50 \mathrm{~Hz}$ that can be written to tape for offline analysis. The Level 1 trigger system is comprised of custom hardware that looks for events consistent with muons, electrons, and jets. Level 1 reduces the 1.7 MHz interaction rate to about $1.5 \mathrm{kHz}$. Events that pass the Level 1 trigger are sent to Level 2, which uses Alpha processors and more detailed hit information to further reject events, with an output rate of about $800 \mathrm{~Hz}$. Upon a Level 2 Accept, data from all front end systems are sent to a farm of PC's that run an online version of the event reconstruction code. Filters based on physics objects are applied to reduce the rate to $50 \mathrm{~Hz}$.

\subsubsection{Level 1 Trigger}

The Level 1 trigger is hardware based and produces a trigger decision for every live 396 ns accelerator bunch crossing. The latency of the Level 1 system is $3.35 \mu$ s, thus a pipelined architecture is used to avoid deadtime. The Level 1 trigger framework receives triggers from the calorimeter, muon, and central tracking Level 1 subsystems and forms global trigger decisions based on these inputs and programmed trigger conditions.

Level 1 Calorimeter Trigger The $0.1 \times 0.1 \eta-\phi$ towers in the calorimeter are combined into $0.2 \times 0.2$ trigger towers on the BLS cards. The analog signals for the electromagnetic and hadronic portion are sent to the Level 1 Calorimeter trigger. The trigger converts the analog signals into $E_{T}$ values for the EM towers (for electron triggering) and for the EM + hadronic towers (for jet triggers). Triggers can be 
formed by requiring a certain number of towers above a set threshold.

Level 1 Central Track Trigger The Level 1 Central Track Trigger (L1CTT) uses hits generated from the axial layers of the Central Fiber Tracker to form axial tracks. The tracks are first formed in $4.5^{\circ}$ sectors in $\phi$. Hits from the individual fibers are first grouped into doublets. Next, the data are compared to defined track equations for four $P_{T}$ thresholds. Up to six tracks for each $P_{T}$ threshold are found in each sector.

The six highest $P_{T}$ tracks from each sector are then sent to an octant trigger card, which sums the 10 sectors in a given octant. The tracks are also sent to the Level 1 Muon Trigger (L1MU), where they can be matched to muon scintillator hits. The octant decisions are sent to a final stage of triggering, where the global L1CTT terms are found. These are sent to a Trigger Manager card for download and transmission to the Trigger Framework.

Level 1 Muon Trigger The Level 1 Muon (L1MU) trigger uses hits from all muon detectors and tracks from the L1CTT to look for hits consistent with muons. The L1MU trigger is described in detail in Chapters 4 and 5.

\subsubsection{Level 2 Trigger}

The Level 2 trigger uses custom hardware based on the DEC Alpha chip and more event information to reduce the Level 1 Accept rate by a factor of two [32]. Each sub-system (Central Tracking, Calorimeter, and Muon) has pre-processors that form triggers that are then passed to a global processor for formation of the global Level 2 trigger.

In the calorimeter, the trigger towers from Level 1 are passed into the calorimeter pre-processor. The Level 2 algorithms perform clustering on the towers for improved electron and jet identification. EM towers are combined into $1 \times 2$ tower regions, while Level 2 jets are formed from 5 x 5 towers. 
In the muon system, wire and scintillator times are sent along with the hit information for improved track segment finding. This track finding is done with Digital Signal Processors on custom hardware cards called the Second Level Input Card (SLIC), often referred to as the muon pre-processors. The output of the SLIC is sent to the muon processors (central and forward) to form the Level 2 muon triggers.

\subsubsection{Level 3 Trigger and Data Acquisition}

The Level 3 trigger uses a collection of approximately 100 farm nodes running Linux and an online version of the event reconstruction code to reduce the Level 2 Accept rate to $50 \mathrm{~Hz}$ [33]. Each event is sent to a single farm node, where event reconstruction takes place. Filters are then run that can select events based on physics quantities (particle momenta, isolation, etc.).

On a Level 2 Accept, data from every detector front end are collected in one of roughly 80 readout crates. Each readout crate has a Single Board Computer (SBC) that reads the event data and sends it over $100 \mathrm{Mbit} / \mathrm{s}$ copper Ethernet to one of four Cisco 2948G switches, which have $48100 \mathrm{Mbit} / \mathrm{s}$ inputs and two Gbit/s outputs. The eight Gbit/s optical lines leave the movable counting house and enter a Cisco 6509 switch that sends the event to the appropriate farm node over $100 \mathrm{Mbit} / \mathrm{s}$ copper Ethernet.

The farm nodes are dual processor $1 \mathrm{GHz}$ Pentium III PC's with 1 GB of RAM. Each farm node handles events at a rate of 10-20 Hz. Events that pass a Level 3 filter are sent from the farm node to a collector, which then sends the events over Gbit/s optical Ethernet to the Feynman Computing Center, where the events are written to tape for offline analysis. 


\section{CHAPter 4 \\ LeVel 1 Muon Trigger}

The Level 1 Muon (L1MU) trigger looks for patterns consistent with muons using hits from muon wire chambers, muon scintillation counters, and tracks from the Level 1 Central Track Trigger (L1CTT). Field Programmable Gate Arrays (FPGA's) are used to perform combinatorial logic on roughly 60,000 muon channels and up to 480 tracks from L1CTT for every bunch crossing. Data from the detector front-ends are transmitted on custom Gbit/s serial links over standard coaxial cable. The serial link receivers and FPGA's are located on VME cards that reside in four custom VME crates on the detector platform.

The muon system (and L1MU) is divided into a central, north, and south region. Each region is further divided into octants. Front-end data from each octant are processed by two L1MU trigger cards (Figure 4.1). The scintillator trigger cards (MTC05) match central tracks to muon scintillator hits while the wire trigger cards (MTC10) match wire-confirmed scintillator hits between the three layers of the muon system. The octant decisions from each MTC05/MTC10 pair in a region are summed in the Muon Trigger Crate Managers (MTCM's) and sent to a global trigger manager (MTM). The MTM forms 256 global L1MU triggers and sends up to 32 of these to the Trigger Framework for inclusion in the Level 1 physics trigger. The download of the specific triggers is handled via EPICS software.

All trigger cards (MTC05, MTC10, MTM) are based on a common mother board (MTCxx) that handles the synchronization and buffering of the data. When a Level 1 Accept is issued for a given event, the trigger decisions are sent to the Level 2 Muon Trigger (L2MU) via a serial link (Cyprus Hotlink) at 160 Mbits/s. Upon receiving a Level 2 Accept, all octant triggers and status and error flags are sent to the Level 3 


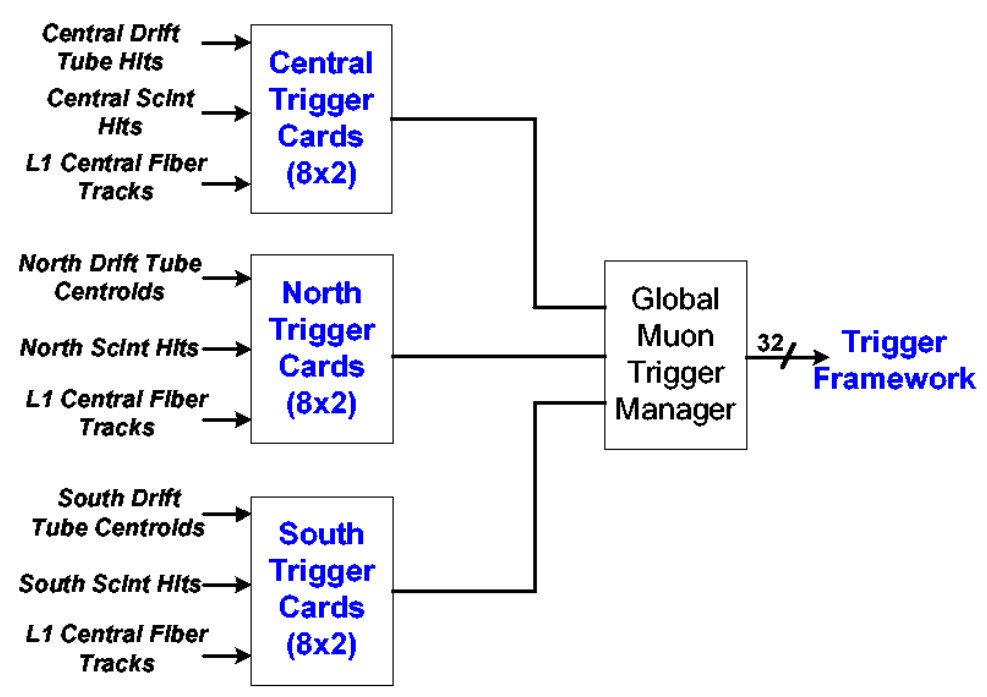

Figure 4.1. The Level 1 Muon trigger system.

system for inclusion in the event readout, also over a Cyprus Hotlink serial connection. Optionally, all input data and control register settings can also be sent for debugging purposes.

\subsection{Gbit/s Serial Links}

All detector inputs to the L1MU trigger use custom Gbit/s serial links that transmit data over Times Microwave LMR-200 coaxial copper cable [34][35]. The links are based on the AMCC S2042/S2043 fiber-optic transmitter/receiver pair. The individual transmitters and receivers are implemented on $1.5 \times 2.2$ in $^{2}$ daughter cards with a 30-pin connector for data and control signals. An additional 10-pin connector is used for system programming via JTAG.

To compensate for signal degradation from the coaxial cable, an amplifier-equalizer circuit is employed on the receivers. This equalizes the incoming signals to correct for the frequency dependent attenuation of the cable, then amplifies the output of the equalizer before it is input to the receiver chip. Figure 4.2 shows the incoming signals before and after the amplifier/equalizer circuit. 

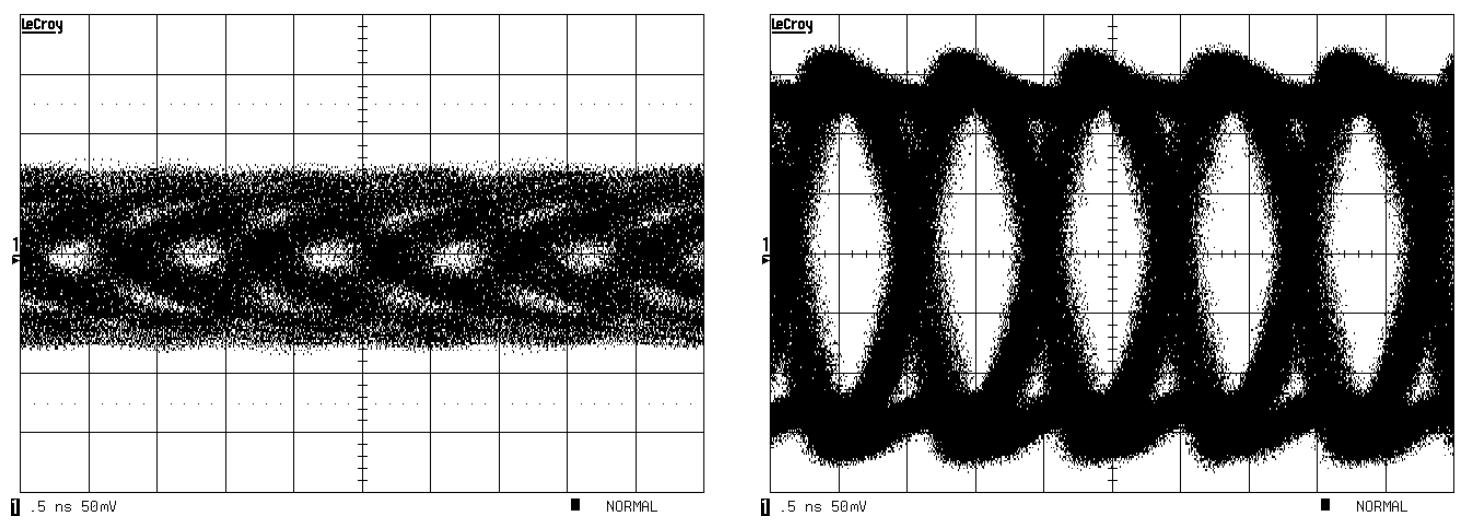

Figure 4.2. The eye pattern before and after the amplifier/equalizer circuit. The horizontal and vertical scales are 500 ps and $50 \mathrm{mV}$, respectively.

Each transmitter/receiver pair can send 16 bits of data for every $18.8 \mathrm{~ns}$ RF (radio frequency) clock, which is the RF frequency of the Tevatron. The data are encoded using an 8b/10b protocol and an encoded RF clock is sent with the data stream. This allows the receiver to decode an $\mathrm{RF}$ clock that is aligned with the data and a bit that indicates whether the word is a data or idle character.

Every 132 ns bunch crossing is divided into seven RF strobes. Data are sent on the first six strobes while a parity error word is sent on the seventh. Thus, each link carries:

$$
16 \frac{\text { bits }}{\text { strobe }} \times 6 \frac{\text { strobes }}{B C}=96 \frac{\text { bits }}{B C}
$$

bits of data per bunch crossing. On the seventh strobe, the value of the parity word calculated on the receiver is compared with the value that was calculated and sent by the transmitter. A disagreement indicates that there was a transmission error on at least one of the seven words and an error bit is raised by the receiver and sent to the MTCxx.

Many of the front end signals must be transmitted to more than one trigger card. A splitter card is used to split an incoming signal three ways. Like the receivers, the 
splitter card has a cable equalizer-amplifier circuit embedded in it. In the case of the splitters, the gain of the amplifier can be adjusted.

\subsection{Octant Trigger Cards}

The heart of the L1MU trigger is the octant trigger card. Each octant trigger card is comprised of an MTCxx motherboard and a daughter flavor card. The MTCxx is a 9U x $400 \mathrm{~mm}$ VME card with a custom J2 VME connector [36]. Each MTCxx has sixteen serial receivers that receive data from the various detector front ends. The synchronization of the inputs is handled with FIFO's, and all input data and trigger decisions are buffered in Dual Port Memories (DPM's) on the MTCxx. The flavor card performs combinatorial logic on the inputs and produces the octant trigger decisions. The different trigger cards (MTC05, MTC10, MTM) are formed by changing the flavor card on the MTCxx motherboard. The three types of flavor cards share a common interface with the MTCxx, but have different FPGA's and card layout to accommodate the different inputs.

\subsubsection{Event Synchronization}

The synchronization of the inputs from various muon and L1CTT front ends is handled by the MTCxx. The data from the various front end systems arrive at an octant trigger card asynchronously due to different drift times, front end processing times, and cable lengths. To synchronize the data for a given event, data from each receiver are written directly into a FIFO. These FIFO's are reset during a system reset and

are initially empty. As each FIFO fills with data from the receiver, a bit from the FIFO indicating it is empty is dropped. Each FIFO stores the data from a receiver until all FIFO's have received data. Next, the data are read from the FIFO's, stored in Dual Port Memory, and sent to the flavor card for trigger formation. 
The front end systems send idle characters (K28.5) during the $2.2 \mu$ s accelerator synch gap (section 5.1.1) during which no collisions occur. During this time, data in the FIFO's are still read out but no new data are written in. Thus, all FIFO's go empty during the synch gap if the difference in latency between the longest and shortest inputs is less than $2.2 \mu \mathrm{s}$. In the L1MU trigger, this difference has been measured to be about 660 ns. Thus when data begin arriving after the synch gap, the FIFO's are again all empty and the same synchronization scheme applies.

\subsubsection{Data Buffering}

In addition to synchronizing the data for a given event, the MTCxx's also buffer the input data and trigger decisions pending global Level 1 and Level 2 trigger decisions. All buffering in the L1MU trigger is performed by writing the data for a given event into Dual Port Memories and then moving a pointer to the data through a series of FIFO's depending on the event status. All pointers are in one of four FIFO's:

- Empty - Available for next event

- L1 Pending - Events waiting for a Level 1 decision

- L2 Pending - Events that have passed Level 1 and are waiting for a Level 2 decision

- Transfer Pending - Events that have passed Level 2 and are awaiting readout to Level 3

When the data for the first event are read from the input FIFO's, an available DPM memory location is read from the Empty FIFO and the data are written into DPM's at that address. The pointer is then moved to the Level 1 Pending FIFO. This pointer is also stored in a CPLD while the L1MU trigger decision for that event 
is being formed. Once the L1MU trigger decision has been formed, the same pointer is used to store the decision in a separate DPM.

A Level 1 Accept issued by the Trigger Framework moves the pointer to the Level 2 Pending FIFO and also makes the address available for use in sending data to the Level 2 system. Upon a Level 2 Accept from the Trigger Framework, the pointer is moved into the Transfer Pending FIFO, where it is used to send data for that event to the Level 3 system for readout. When all of the data for a given event have been sent to Level 3, or a Level 1 or Level 2 Reject has been received from the Trigger Framework, the pointer is put back into the empty list and that location in memory is available for a future event. Every event on the trigger card is also marked with a bunch crossing number as a check of event synchronization.

\subsection{Trigger Crate}

Triggers for each region of the muon system (central, north, and south) are formed by sixteen octant trigger cards in one VME crate. Each trigger crate (Figure 4.3) has sixteen trigger cards that form the octant triggers, a Muon Trigger Crate Manager (MTCM) that combines the octant triggers into regional triggers, and a Motorola MVME-162 processor that acts as bus master. Details of the MTCM can be found in $[37]$.

Event synchronization for the sixteen MTCxx's in a crate is handled in a similar fashion to the synchronization on a given trigger card. When trigger decisions for the first bunch crossing have been formed on a given trigger card, they are written into a FIFO called the trigger decision FIFO that is initially empty. When all trigger cards in the crate have formed the trigger decisions for the first bunch crossing, the Crate Manager begins reading the trigger decisions from the FIFO's on the trigger cards.

The trigger decisions from the sixteen trigger cards are combined on the MTCM to form regional trigger decisions. The octant trigger decisions are also buffered on 


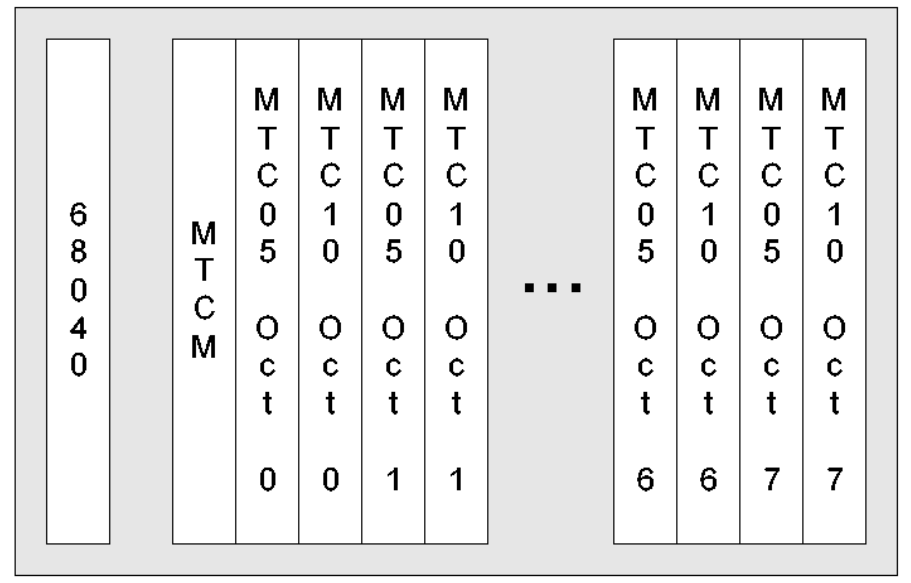

Figure 4.3. Level 1 Muon trigger crate. Each crate produces trigger decisions for one region (central, north, south) of the detector. A total of sixteen trigger cards are used to form octant triggers that are combined on the MTCM.

the MTCM in the same way as the data on the MTCxx's while awaiting the Level 1 and Level 2 trigger decisions from the Trigger Framework. The regional trigger decisions are sent to the Trigger Manager (TM) crate, which combines the regional decisions and forms the global L1MU trigger decisions. The TM crate consists of a single Muon Trigger Manager card (MTCxx with an MTM flavor card), a Crate Manager, and a Motorola 68040 processor.

The triggers from the three regions are sent to the MTM card with the same Gbit/s serial links used by the front ends and are synchronized in the same manner as the input data at the octant trigger cards. A total of 256 global L1MU triggers are formed from the regional trigger decisions, up to 32 of which are sent to the Trigger Framework for inclusion in the global Level 1 trigger. The 32 trigger bits are sent to the TF as differential ECL signals on two twist and flat 40c cables. A clock signal and a synch gap signal are also sent with the trigger decisions to mark the timing and bunch crossing number for the triggers.

In addition to regional trigger formation, the MTCM's in each crate also receive timing and control information (RF clock, trigger decisions) from the Trigger Frame- 
work via the Muon Readout Card and distribute this information to the trigger cards over the backplane. The MTCM's also build the messages sent to the Level 2 and Level 3 trigger systems that include the L1MU octant trigger decisions and status and error information for the event. Additionally, all input data received by the trigger cards can be sent to Level 3.

Every trigger crate has a Motorola MVME-162 68040 processor in the first slot that acts as bus master. The MVME-162 uses standard Ethernet and the VxWorks operating system. In practice, the MVME-162 is used to download FPGA programs used by the flavor cards for trigger formation and perform necessary setups after a power cycle. The MVME-162 also monitors status and error registers on the trigger cards and receives download requests for the Trigger Manager using EPICS.

\subsection{Trigger Logic}

The combinatorial logic used to form triggers from the front end data is performed by FPGA's located on the various flavor cards. The flavor cards connect to the MTCxx through seven Samtec connectors. The programming files for the FPGA's are stored in non-volatile flash memory on the MTCxx's for fast configuration of the FPGA's after a power cycle.

The inputs to the flavor cards are 256 input bits (16 bits/receiver x 16 receivers) along with the RF clock, bunch crossing (BC) clock, and the start processing signal. There are a total of 36 output bits that carry the octant trigger decisions from the flavor cards to the MTCxx.

\subsubsection{MTC05}

The MTC05 flavor card matches tracks from the Level 1 Central Track Trigger (L1CTT) to hits in the muon scintillator system. Each MTC05 receives 12 input cables from the L1CTT, with each input covering $4.5^{\circ}$ of the central tracker. This 


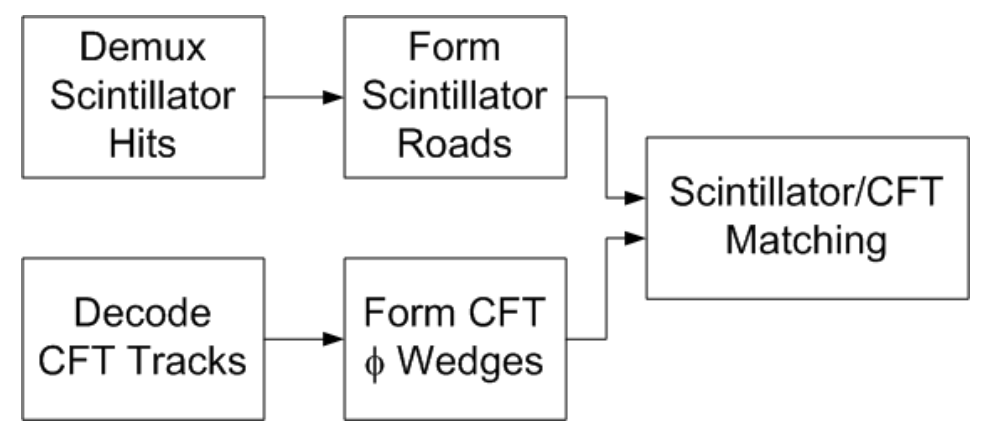

Figure 4.4. MTC05 trigger logic.

gives full coverage for the octant plus $4.5^{\circ}$ of overlap on each side. The remaining four input cables carry the muon scintillator hit information.

Each input from the L1CTT carries the six highest $\mathrm{P}_{\mathrm{T}}$ tracks from a given $4.5^{\circ}$ sector. Each track has a phi position in the outer layer of the central tracker, a $\mathrm{P}_{\mathrm{T}}$ value, and a curvature bit that indicates which direction the track was bent by the magnetic field. The $\mathrm{P}_{\mathrm{T}}$ value is divided into four bins, or thresholds. The thresholds are in differential $\mathrm{P}_{\mathrm{T}}$ bins from 1.5 to $3 \mathrm{GeV} / \mathrm{c}\left(\mathrm{P}_{\mathrm{T}} 1\right), 3$ to $5 \mathrm{GeV} / \mathrm{c}\left(\mathrm{P}_{\mathrm{T}} 2\right), 5$ to 10 $\mathrm{GeV} / \mathrm{c}\left(\mathrm{P}_{\mathrm{T}} 3\right)$, and greater than $10 \mathrm{GeV} / \mathrm{c}\left(\mathrm{P}_{\mathrm{T}} 4\right)$. Details of the track format are in Appendix A.1.1.

The triggers formed by the MTC05 are the number of scintillator tracks found with matching central tracks for the various $\mathrm{P}_{\mathrm{T}}$ thresholds. Loose triggers match A-layer scintillator hits to central tracks while tight triggers first look for a two layer scintillator track, or road, and then match it to a central track. In addition, scintillator-only triggers are formed that do not require the matching central track.

Figure 4.4 shows a schematic view of the logic. First, tracks from the L1CTT are decoded and used to form ' $\phi$-wedges'. These $\phi$-wedges correspond to the range of $\phi$ positions in the outer layer of the central tracker that match each scintillation counter. The wedges are formed for all four $\mathrm{P}_{\mathrm{T}}$ thresholds and both values of the sign. In parallel, the scintillator hit information is demultiplexed and scintillator roads are formed in the three layers. The roads are anchored in the A-layer and are typically 
$\mathrm{A} \& \mathrm{C}$ in the central region and $\mathrm{A} \& \mathrm{~B}$ in the forward region. Details of the scintillator roads in the different regions and octants can be found in [38].

The scintillator roads are then matched to the $\phi$-wedges formed for the four $\mathrm{P}_{\mathrm{T}}$ thresholds. The number of scintillator roads with a matching central track are counted (up to two). Finally, the triggers for the four differential $\mathrm{P}_{\mathrm{T}}$ thresholds are integrated so the final triggers correspond to a minimum $\mathrm{P}_{\mathrm{T}}$ threshold and greater. This means a trigger formed by matching a scintillator roads to a $10 \mathrm{GeV} / \mathrm{c}$ track will also fire the lower $\mathrm{P}_{\mathrm{T}}$ threshold triggers as well.

Counting of triggers requires some care. Scintillator roads are first anchored by their $\eta-\phi$ index in one layer. A dimuon trigger, for example, requires at least two scintillator roads, separated by at least one counter in $\eta$ or $\phi$ in the anchor layer. This separation in $\eta-\phi$ space effectively imposes a minimum $\Delta \mathrm{R}=\sqrt{(\Delta \eta)^{2}+(\Delta \phi)^{2}}$ cut of roughly 0.5 in the central region and 0.25 in the forward region. The difference is due to the different $\eta$ segmentation of the counters in the two regions.

The MTC05 logic is implemented on four 484-pin Altera ACEX 1K100 FPGA's. One FPGA forms scintillator roads, two are used to match L1CTT tracks to the scintillator roads, and the fourth integrates the four $\mathrm{P}_{\mathrm{T}}$ bins and sends triggers to the MTCxx. The total latency for the MTC05 logic is $15 \times 18.8 \mathrm{~ns}=282 \mathrm{~ns}$ and is summarized in Table 4.1.

\subsubsection{MTC10}

The MTC10 logic forms triggers based on scintillator hits confirmed with wire chamber track stubs that are matched between layers. In the central region, thirteen input cables are used to carry PDT hits, while the remaining three cables carry scintillator hit information. In the forward region, eleven input cables are used for MDT centroids formed on the Centroid cards (MCEN's) and four inputs are used for the scintillators. Details of the inputs are in Appendix A.2.3. 


\begin{tabular}{|l|l|l|}
\hline$R F$ Clock & Track & Scintillator \\
\hline 0 & Decode track 0 & demux scint 0 \\
\hline 1 & Form first wedge & demux scint 1 \\
\hline 2 & Latch first wedge & demux scint 2 \\
\hline 3 & First track into $\phi$-wedge & demux scint 3 \\
\hline 4 & Second track into $\phi$-wedge & demux scint 4 \\
\hline 5 & Third track into $\phi$-wedge & demux scint 5 \\
\hline 6 & Fourth track into $\phi$-wedge & latch output of demux \\
\hline 7 & Fifth track into $\phi$-wedge & Find scint roads \\
\hline 8 & Sixth track into $\phi$-wedge & Find scint roads \\
\hline 9 & Latch tracks out of $\phi$-wedge & Latch to P $_{\mathrm{T}}$ chips \\
\hline 10 & Combine $\phi$-wedges and scint. roads \\
\hline 11 & Form triggers, add to 2 \\
\hline 12 & Latch to summary \\
\hline 13 & Summary Logic \\
\hline 14 & Latch to MTCxx \\
\hline 15 & Data at MTCxx \\
\hline
\end{tabular}

TABLE 4.1. MTC05 trigger latency.

A schematic of the MTC10 logic is shown in Figure 4.5. In the central region, wire hits are demultiplexed and centroids are formed. In the forward region, the centroids from the MCEN cards are decoded and OR'd by two. Next, demultiplexed scintillator hits are confirmed by the centroids by matching the position of the scintillator hit and centroid. A range of centroid positions that overlap each scintillator, determined from Monte Carlo, is used to confirm a given scintillator hit. In the central region, where the maximum drift time for the PDT's spans several bunch crossings, the confirmation eliminates ambiguity in the proper bunch crossing for the centroid. In both regions, using confirmed scintillator hits for the wire triggers allows MTC10 to use the same matching equations between layers and the same counting scheme as the MTC05. The outputs are loose (A-layer) and tight (two layer coincidence) triggers.

The MTC10 logic uses three 256-pin ACEX 10k50 FPGA's to form the centroids and confirm the scintillator hits (one for each layer), one 256-pin 1K100 FPGA to 


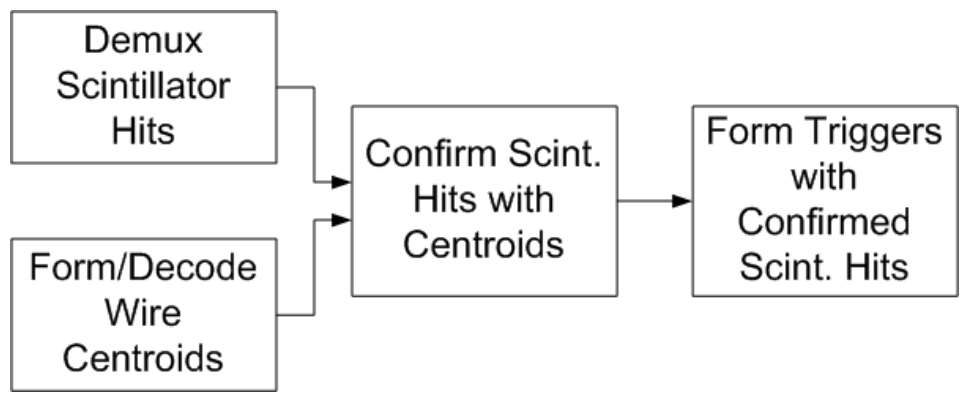

FiguRE 4.5. MTC10 trigger logic.

form triggers between the different layers, and one 256-pin 1K30 FPGA to align the triggers and send them to the MTCxx. Two additional 256-pin 1K100 FPGA's are available to form triggers but are not necessary with the current algorithms. The latency in the central region is $31 \times 18.8 \mathrm{~ns}=583 \mathrm{~ns}$, while the forward logic requires $25 \times 18.8 \mathrm{~ns}=470 \mathrm{~ns}$. The latency for the central and forward logic is summarized in Tables 4.2 and 4.3 .

\subsubsection{Octant Trigger Counters}

The octant trigger decision information is organized as 2-bit counters. The total number of 2-bit counters that can be sent from each octant to the Crate Manager for inclusion in the regional trigger is 18 (36 bits total). These 18 counters are shared between the MTC05 and MTC10 in each octant. Tables 4.4 and 4.5 give the definition of these counters for the central and forward regions as of August, 2004. Details of triggers for all octants are given in Table 4.6 and in [38].

In addition to the physics triggers, several triggers are included to monitor rates in single layers. In the central region, the ' $\mathrm{P}_{\mathrm{T}} 4$ Only' trigger gives the rate of high $\mathrm{P}_{\mathrm{T}}$ tracks sent to the L1MU trigger and can be compared to the rate from the L1CTT trigger. In both regions, the scintillator and wire loose triggers give the A-layer scintillator and wire rates respectively. The logical OR of the B- and C-layer scintillator rates is available in both regions, while the B- and C-layer wire rates can be monitored 


\begin{tabular}{|l|l|}
\hline$R F$ Clock & Step \\
\hline $0-5$ & Demux PDT and scint hits \\
\hline 7 & Latch output of demux \\
\hline $8-10$ & Find centroids, confirm scint hits \\
\hline 11 & Latch confirmed scint hits \\
\hline 12 & Mux 0 in \\
\hline 13 & Mux 0 out \\
\hline 14 & demux 0 at trigger 1 chip \\
\hline $15-19$ & demux 1-5 \\
\hline 20 & Latch output of demux \\
\hline $21-23$ & Form triggers, count to 2 \\
\hline 24 & Latch trigger output to summary \\
\hline 25 & Nothing \\
\hline 26 & Latch triggers into summary \\
\hline 27 & Summary logic \\
\hline 28 & Latch data to MTCxx \\
\hline 29 & Data appear at MTCxx \\
\hline
\end{tabular}

TABLE 4.2. CF MTC10 trigger latency.

\begin{tabular}{|l|l|}
\hline RF Clock & Step \\
\hline $0-5$ & Demux PDT and scint hits \\
\hline 6 & Latch output of demux \\
\hline 7 & Confirm scint hits \\
\hline 8 & Latch confirmed scint hits to mux \\
\hline 9 & Mux 0 in \\
\hline 10 & Mux 0 out \\
\hline 11 & demux 0 at trigger 1 chip \\
\hline $12-16$ & demux 1-5 \\
\hline 17 & Latch output of demux \\
\hline $18-20$ & Form triggers, count to 2 \\
\hline 21 & Latch trigger output to summary \\
\hline 22 & Summary logic, latch to MTCxx \\
\hline 23 & Data appear at MTCxx \\
\hline
\end{tabular}

TABLE 4.3. EF MTC10 trigger latency. 


\begin{tabular}{|c|l|l|l|}
\hline Counter & Card & Name & Description \\
\hline 0 & MTC05 & $\mathrm{P}_{\mathrm{T}} 1+$ Loose & $\mathrm{P}_{\mathrm{T}} 1+$ track matched to $\mathrm{A} \phi$ \\
\hline 1 & MTC05 & $\mathrm{P}_{\mathrm{T}} 1$ - Loose & $\mathrm{P}_{\mathrm{T}} 1-$ track matched to $\mathrm{A} \phi$ \\
\hline 2 & MTC05 & $\mathrm{P}_{\mathrm{T}} 2$ Loose & $\mathrm{P}_{\mathrm{T}} 2+$ track matched to $\mathrm{A} \phi$ \\
\hline 3 & MTC05 & $\mathrm{P}_{\mathrm{T}} 2$ - Loose & $\mathrm{P}_{\mathrm{T}} 2-$ track matched to $\mathrm{A} \phi$ \\
\hline 4 & MTC05 & $\mathrm{P}_{\mathrm{T}} 3$ Loose & $\mathrm{P}_{\mathrm{T}} 3$ track matched to A $\phi$ \\
\hline 5 & MTC05 & $\mathrm{P}_{\mathrm{T}} 4$ Loose & $\mathrm{P}_{\mathrm{T}} 4$ track matched to $\mathrm{A} \phi$ \\
\hline 6 & MTC05 & $\mathrm{P}_{\mathrm{T}} 2$ Tight & $\mathrm{P}_{\mathrm{T}} 2$ track matched to A $\phi$ CMSC \\
\hline 7 & MTC05 & $\mathrm{P}_{\mathrm{T}} 3$ Tight & $\mathrm{P}_{\mathrm{T}} 3$ track matched to A $\phi$ CMSC \\
\hline 8 & MTC05 & $\mathrm{P}_{\mathrm{T}} 4$ Tight & $\mathrm{P}_{\mathrm{T}} 4$ track matched to A $\phi$ CMSC \\
\hline 9 & MTC05 & Scintillator Loose & A $\phi$ only \\
\hline 10 & MTC05 & CMSC Loose & CMSC only \\
\hline 11 & MTC05 & Scintillator Tight & A $\phi$ CMSC \\
\hline 12 & MTC05 & $\mathrm{P}_{\mathrm{T}} 4$ Only & $\mathrm{P}_{\mathrm{T}} 4$ Track \\
\hline 13 & MTC05 & MTC05 BOT & BOT(bit 0) \\
\hline 14 & MTC10 & Wire Monitor & B-or (bit 0), C-or (bit 1) \\
\hline 15 & MTC10 & Wire Loose & A PDT \\
\hline 16 & MTC10 & Wire Tight & AB or AC PDT \\
\hline 17 & MTC10 & MTC10 BOT & BOT(bit 0) \\
\hline
\end{tabular}

TABLE 4.4. Central 2-bit octant trigger decision counters. 


\begin{tabular}{|c|l|l|l|}
\hline Counter & Card & Name & Description \\
\hline 0 & MTC05 & $\mathrm{P}_{\mathrm{T}} 1$ Loose & $\mathrm{P}_{\mathrm{T}} 1$ track matched to Apix \\
\hline 1 & MTC05 & $\mathrm{P}_{\mathrm{T}} 2$ Loose & $\mathrm{P}_{\mathrm{T}} 2$ track matched to Apix \\
\hline 2 & MTC05 & $\mathrm{P}_{\mathrm{T}} 3$ Loose & $\mathrm{P}_{\mathrm{T}} 3$ track matched to Apix \\
\hline 3 & MTC05 & $\mathrm{P}_{\mathrm{T}} 4$ Loose & $\mathrm{P}_{\mathrm{T}} 4$ track matched to Apix \\
\hline 4 & MTC05 & $\mathrm{P}_{\mathrm{T}} 2$ Tight & $\mathrm{P}_{\mathrm{T}} 2$ track matched to AB pix \\
\hline 5 & MTC05 & $\mathrm{P}_{\mathrm{T}} 3$ Tight & $\mathrm{P}_{\mathrm{T}} 3$ track matched to AB pix \\
\hline 6 & MTC05 & $\mathrm{P}_{\mathrm{T}} 4$ Tight & $\mathrm{P}_{\mathrm{T}} 4$ track matched to AB pix \\
\hline 7 & MTC05 & $\mathrm{P}_{\mathrm{T}} \mathrm{x}$ Loose & Apix, $1.0<|\eta|<1.6$ \\
\hline 8 & MTC05 & $\mathrm{P}_{\mathrm{T}} \mathrm{x}$ Loose & Apix, $1.6<|\eta|<2.0$ \\
\hline 9 & MTC05 & $\mathrm{P}_{\mathrm{T}} \mathrm{x}$ Tight & ABpix, $1.0<|\eta|<1.6$ \\
\hline 10 & MTC05 & $\mathrm{P}_{\mathrm{T}} \mathrm{x}$ Tight & ABpix, $1.6<|\eta|<2.0$ \\
\hline 11 & MTC05 & MTC05 BOT & BOT (bit 0$),$ B or Cpix (bit 1) \\
\hline 12 & MTC10 & Wire Loose & A-MDT $1.0<|\eta|<1.6$ \\
\hline 13 & MTC10 & Wire Loose & A-MDT $1.6<|\eta|<2.0$ \\
\hline 14 & MTC10 & Wire Tight & AB-MDT $1.0<|\eta|<1.6$ \\
\hline 15 & MTC10 & Wire Tight & AB-MDT $1.6<|\eta|<2.0$ \\
\hline 16 & MTC10 & Wire Monitor & B-or (bit 0$),$ C-or $($ bit 1$)$ \\
\hline 17 & MTC10 & MTC10 BOT & BOT(bit 0$)$ \\
\hline
\end{tabular}

TABLE 4.5. Forward 2-bit octant trigger decision counters.

\begin{tabular}{|l|l|l|l|l|l|}
\hline Octant & $P_{\mathrm{T}}$ & Quality & Roads & Anchor & Count Two \\
\hline Central & & & & & \\
\hline $0-3$ & $\mathrm{P}_{\mathrm{T}} 1-4, \mathrm{P}_{\mathrm{T}} \mathrm{x}$ & Loose & $\mathrm{A}$ & $\mathrm{A}$ & $\mathrm{A}$ \\
\hline $0-3$ & $\mathrm{P}_{\mathrm{T}} 2-4, \mathrm{P}_{\mathrm{T}} \mathrm{x}$ & Tight & $\mathrm{AC}$ & $\mathrm{A}$ & $\mathrm{A}$ \\
\hline $0-3$ & $\mathrm{P}_{\mathrm{T}} \mathrm{x}(\mathrm{Wire})$ & Tight & $\mathrm{AB}$ or $\mathrm{AC}$ & $\mathrm{A}$ & $\mathrm{A}$ \\
\hline 4,7 & $\mathrm{P}_{\mathrm{T}} 1-4, \mathrm{P}_{\mathrm{T}} \mathrm{x}$ & Loose & $\mathrm{A}$ & $\mathrm{A}$ & $\mathrm{A}$ \\
\hline 4,7 & $\mathrm{P}_{\mathrm{T}} 2-4, \mathrm{P}_{\mathrm{T}} \mathrm{X}$ & Tight & $\mathrm{AB}$ or $\mathrm{AC}$ & $\mathrm{A}$ & $\mathrm{A}$ \\
\hline 4,7 & $\mathrm{P}_{\mathrm{T}} \mathrm{x}(\mathrm{Wire})$ & Tight & $\mathrm{AB}$ or $\mathrm{AC}$ & $\mathrm{A}$ & $\mathrm{A}$ \\
\hline 5,6 & $\mathrm{P}_{\mathrm{T}} 1$ & Loose & $\mathrm{A}$ & None & $\mathrm{All}$ \\
\hline 5,6 & $\mathrm{P}_{\mathrm{T}} 2-4, \mathrm{P}_{\mathrm{T}} \mathrm{x}$ & Loose & $\mathrm{A}$ or $\mathrm{B}$ or $\mathrm{C}$ & None & $\mathrm{All}$ \\
\hline 5,6 & $\mathrm{P}_{\mathrm{T}} 2-4, \mathrm{P}_{\mathrm{T}} \mathrm{x}$ & Tight & $\mathrm{AB}$ or $\mathrm{AC}$ or $\mathrm{BC}$ & $\mathrm{A}$ & $\mathrm{A}$ \\
\hline Forward & & & & & \\
\hline $0-7$ & $\mathrm{P}_{\mathrm{T}} 1-4, \mathrm{P}_{\mathrm{T}} \mathrm{x}$ & Loose & $\mathrm{A}$ & $\mathrm{A}$ & $\mathrm{A}$ \\
\hline $0-7$ & $\mathrm{P}_{\mathrm{T}} 2-4, \mathrm{P}_{\mathrm{T}} \mathrm{x}$ & Tight & $\mathrm{AB}$ & $\mathrm{A}$ & $\mathrm{A}$ \\
\hline
\end{tabular}

TABLE 4.6. Details of the loose and tight triggers for the central and forward regions. Additional details can be found in [38]. 
separately.

Finally, a beginning-of-turn (BOT) synchronization trigger that fires on the first bunch crossing of every turn is present for both the MTC05 and MTC10. This trigger is generated from the start-processing signal that is sent to the flavor cards and allows a quick check that the data flow through the trigger system is aligned.

\subsubsection{Crate Manager Triggers}

The Crate Manager in each region receives the octant trigger decisions for every event and combines them into regional trigger decisions. The logic on the Crate Manager forms sums of the octant trigger decision counters and reports this as 18 2-bit regional trigger counters to the MTM. The MTCM logic also forms triggers that match MTC05 and MTC10 trigger decisions in an octant (a tight scintillator trigger and a loose wire trigger, for example). In all, 36 2-bit counters are sent from each MTCM to the MTM. Tables 4.7 and 4.8 show the combined triggers for the central and forward regions.

\subsubsection{Trigger Manager Logic}

The Trigger Manager (MTM) is the final stage in forming the L1MU trigger decisions. It receives the regional triggers from the three MTCM's and forms global Level 1 muon triggers that are sent to the Trigger Framework for inclusion in the Level 1 decision. The trigger manager forms 256 triggers, up to 32 of which can be sent to the TF. The downloading of the 32 specific triggers is handled by VME writes to the Trigger Manager and is implemented in the DØ Data Acquisition (DAQ) system via EPICS software running on the MVME-162 processor.

The 256 trigger decisions are named with a unique ten character name. The general scheme is:

$$
\mathrm{mu}\left(\text { multiplicity, } \mathrm{P}_{\mathrm{T}}\right. \text {, eta,scint, wire, option) }
$$




\begin{tabular}{|c|l|}
\hline Counter & Central \\
\hline 0 & $\mathrm{P}_{\mathrm{T}} 1+$ Loose \& Wire Loose \\
\hline 1 & $\mathrm{P}_{\mathrm{T}} 1+$ Loose \& Wire Loose \\
\hline 2 & $\mathrm{P}_{\mathrm{T}} 2+$ Loose \& Wire Loose \\
\hline 3 & $\mathrm{P}_{\mathrm{T}} 2-$ Loose \& Wire Loose \\
\hline 4 & $\mathrm{P}_{\mathrm{T}} 3$ Loose \& Wire Loose \\
\hline 5 & $\mathrm{P}_{\mathrm{T}} 4$ Loose \& Wire Loose \\
\hline 6 & $\mathrm{P}_{\mathrm{T}} \mathrm{x}$ Loose \& Wire Loose \\
\hline 7 & $\mathrm{P}_{\mathrm{T}} 2$ Tight \& Wire Tight \\
\hline 8 & $\mathrm{P}_{\mathrm{T}} 3$ Tight \& Wire Tight \\
\hline 9 & $\mathrm{P}_{\mathrm{T}} 4$ Tight \& Wire Tight \\
\hline 10 & $\mathrm{P}_{\mathrm{T}} \mathrm{x}$ Tight \& Wire Tight \\
\hline 11 & $\mathrm{P}_{\mathrm{T}} 2$ Tight \& Wire Loose \\
\hline 12 & $\mathrm{P}_{\mathrm{T}} 3$ Tight \& Wire Loose \\
\hline 13 & $\mathrm{P}_{\mathrm{T}} 4$ Tight \& Wire Loose \\
\hline 14 & $\mathrm{P}_{\mathrm{T}} \mathrm{x}$ Tight \& Wire Loose \\
\hline
\end{tabular}

TABLE 4.7. Central MTC05/MTC10 combined triggers.

\begin{tabular}{|c|l|}
\hline Counter & Description \\
\hline 0 & $\mathrm{P}_{\mathrm{T}} 1$ Loose \& Wire Loose $1.0<|\eta|<1.6$ \\
\hline 1 & $\mathrm{P}_{\mathrm{T}} 2$ Loose \& Wire Loose $1.0<|\eta|<1.6$ \\
\hline 2 & $\mathrm{P}_{\mathrm{T}} 3$ Loose \& Wire Loose $1.0<|\eta|<1.6$ \\
\hline 3 & $\mathrm{P}_{\mathrm{T}} 4$ Loose \& Wire Loose $1.0<|\eta|<1.6$ \\
\hline 4 & $\mathrm{P}_{\mathrm{T}} \mathrm{x}$ Loose \& Wire Loose $1.0<|\eta|<1.6$ \\
\hline 5 & $\mathrm{P}_{\mathrm{T}} 2$ Tight \& Wire Tight $1.0<|\eta|<1.6$ \\
\hline 6 & $\mathrm{P}_{\mathrm{T}} 3$ Tight \& Wire Tight $1.0<|\eta|<1.6$ \\
\hline 7 & $\mathrm{P}_{\mathrm{T}} 4$ Tight \& Wire Tight $1.0<|\eta|<1.6$ \\
\hline 8 & $\mathrm{P}_{\mathrm{T}} \mathrm{x}$ Tight \& Wire Tight $1.0<|\eta|<1.6$ \\
\hline 9 & $\mathrm{P}_{\mathrm{T}} 2$ Tight \& Wire Loose $1.0<|\eta|<1.6$ \\
\hline 10 & $\mathrm{P}_{\mathrm{T}} 3$ Tight \& Wire Loose $1.0<|\eta|<1.6$ \\
\hline 11 & $\mathrm{P}_{\mathrm{T}} 4$ Tight \& Wire Loose $1.0<|\eta|<1.6$ \\
\hline 12 & $\mathrm{P}_{\mathrm{T}} \mathrm{x}$ Tight \& Wire Loose $1.0<|\eta|<1.6$ \\
\hline 13 & $\mathrm{P}_{\mathrm{T}} \mathrm{x}$ Loose \& Wire Loose $1.6<|\eta|<2.0$ \\
\hline 14 & $\mathrm{P}_{\mathrm{T}} \mathrm{x}$ Tight \& Wire Tight $1.6<|\eta|<2.0$ \\
\hline 15 & $\mathrm{P}_{\mathrm{T}} \mathrm{x}$ Tight \& Wire Loose $1.6<|\eta|<2.0$ \\
\hline
\end{tabular}

TABLE 4.8. Forward MTC05/MTC10 combined triggers. 
where multiplicity can be 1 or $2, \mathrm{P}_{\mathrm{T}}$ is the $\mathrm{P}_{\mathrm{T}}$ threshold required, and eta is the approximate coverage of the trigger. The scint term is the quality of the MTC05 decision and the wire term is the quality of the MTC10 decision. The option is used for different types of dimuon and debugging triggers. A full description of the naming scheme is here [39].

\subsection{Certification}

The L1MU trigger has been fully simulated in $\mathrm{C}++$ as part of the $\mathrm{D} \varnothing$ trigger simulator. The L1MU simulator (tsim_l1muo) uses the digitized readout of the muon system and the results of the L1CTT simulator as inputs. Single muon Monte Carlo events are used to determine detector acceptance and trigger algorithm efficiencies.

The hardware is first simulated at the trigger card (MTCxx) level, with four separate classes for the central and forward MTC05 and MTC10 cards. The simulated Crate Manager sums the octant decisions from the trigger card simulation and forms the regional trigger decisions, which are passed to the Trigger Manager to form the global L1MU simulated trigger decisions. In addition, the Crate Manager class collects the octant, regional, and global trigger decisions and simulates the hardware Level 2 and Level 3 messages. Finally, the simulator allows the storage of debugging information, such as the raw muon and L1CTT information and all 256 global L1MU simulated triggers.

During data taking, the simulator is run online on current data and the results are compared to the readout of the hardware triggers. Plots showing the number of triggers fired in each octant as well as the synchronization, or BOT, triggers are made for each run and compared against templates from known good runs. In addition, plots showing the differences between hardware and simulator triggers are formed every hour. The difference plots are formed by comparing the hardware trigger decisions for every octant to the simulated trigger decisions. Differences are plotted separately 
for the case where the hardware trigger fired when the simulated trigger did not and vice-versa. In addition, the 32 bits of global hardware triggers are compared to the simulation.

The current level of agreement between the simulator and the hardware has been studied for the single and dimuon tight scintillator-only triggers and for the loose single muon wire triggers. The agreement for these triggers is better than $1 \%$ in all regions.

\subsection{Performance}

The performance of the muon system and the L1MU trigger has been studied in Monte Carlo events using tsim_l1muo. Single muon Monte Carlo events are used to determine the detector acceptance for medium muons. Acceptance is defined as

$$
A c c=\frac{N_{h i t s}}{N_{M C}}
$$

where $N_{M C}$ is the total number of MC events and $N_{\text {hits }}$ is the number of events with at least the minimum number of detector hits required to reconstruct a medium muon [40]. The requirements used for the acceptance were

- $\geq 1$ A-layer scintillator hit

- $\geq 2$ A-layer wire hits

- $\geq 1$ BC-layer scintillator hit

- $\geq 2$ BC-layer wire hits

Figure 4.6 shows the acceptance for medium muons from Monte Carlo events. The turn-on curve in $\mathrm{P}_{\mathrm{T}}$ comes from low $\mathrm{P}_{\mathrm{T}}$ muons that do not make it through the toroid and thus do not record hits in the B- and C-layers. The dips in $\eta$ in the central $(|\eta|<$ 1.0) come mainly from the limited coverage in the bottom of the detector, which can 

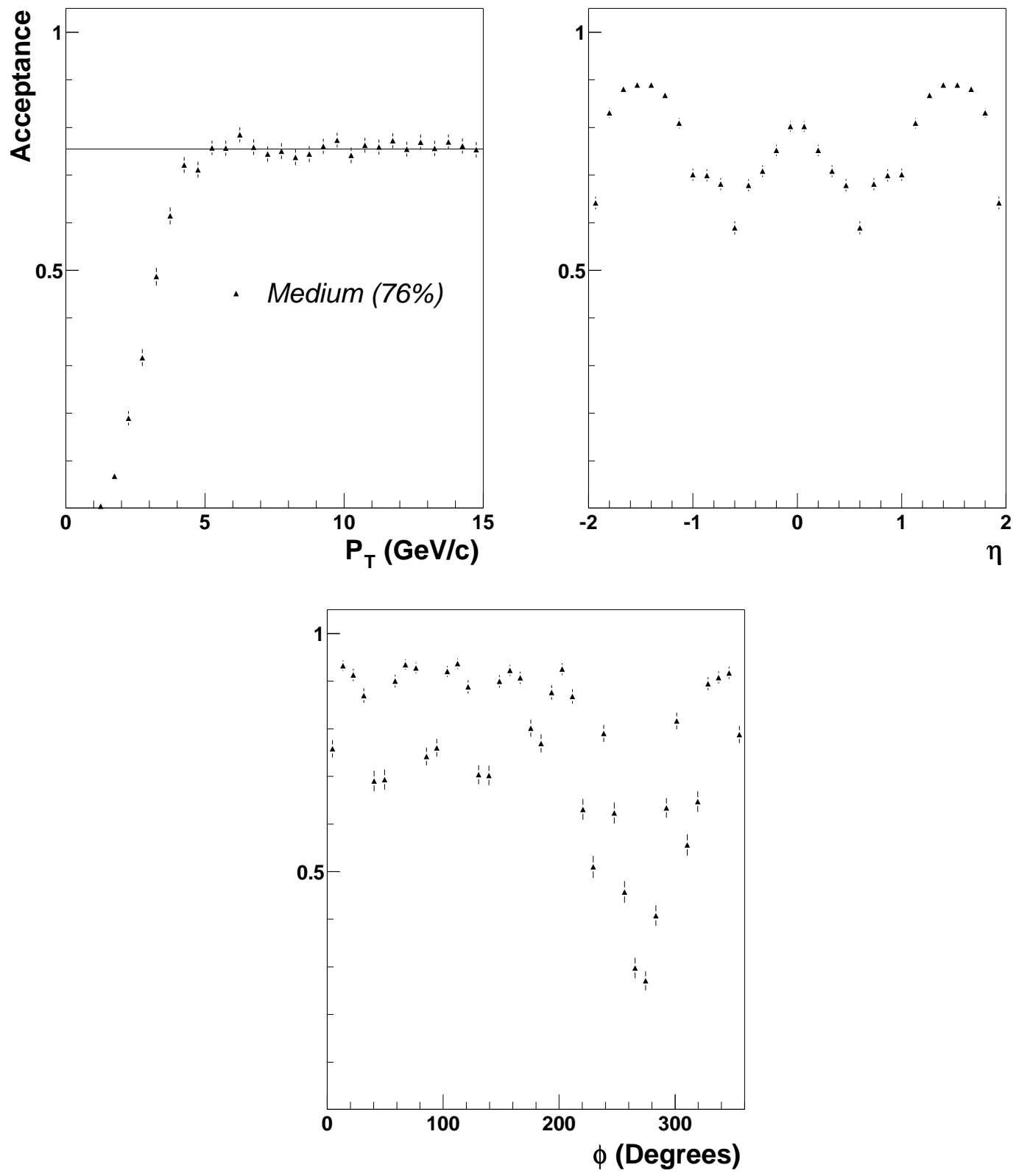

FigurE 4.6. The acceptance of the $\mathrm{D} \varnothing$ muon detector for medium muons. The turnon curve in $\mathrm{P}_{\mathrm{T}}$ is from low energy muons that do not make it through the toroid, while the dips in $\eta$ and $\phi$ come from the limited coverage in the central bottom of the detector. 
be seen in the $\phi$ plot as well. In addition, the dips in $\phi$ every $45^{\circ}$ show the octant boundaries in the muon system.

The muon trigger efficiency is measured with respect to Monte Carlo events that pass the medium muon acceptance criteria. In this case, we define efficiency as

$$
E f f=\frac{N_{\text {Trig }}}{N_{\text {Hit }}}
$$

where $N_{\text {Trig }}$ is the number of events that passed the acceptance criteria and had a simulated trigger, while $N_{H i t}$ is the number of events that passed the acceptance criteria.

Figure 4.7 shows the efficiency for the tight scintillator trigger (mu1ptxatxx) and for the tight scintillator/loose wire trigger (mulptxatlx). The turn-on curve in $\mathrm{P}_{\mathrm{T}}$ comes mainly from the forward region, where low $\mathrm{P}_{\mathrm{T}}$ muons are bent outside of the trigger roads in the toroid. The dips in $\eta$ around -1.0 and 1.0 are due to muons that cross from the central to forward region. Similarly, the dips in $\phi$ every $45^{\circ}$ are due to muons that cross octant boundaries. The fit to the $\mathrm{P}_{\mathrm{T}}$ distribution is for muons with $P_{T}>11 \mathrm{GeV} / \mathrm{c}$, while the $\eta$ and $\phi$ distributions are from Monte Carlo muons with $\mathrm{P}_{\mathrm{T}}=11 \mathrm{GeV} / \mathrm{c}$.

The efficiency has also been measured from collider data. In this case, the bias due to muon triggers is removed by selecting events from jet and electron triggers. The event selection was:

- Single medium muon

- Match to 3D central track

- Pass cosmic rejection cuts (based on differences in scintillator times)

The efficiency is

$$
E f f=\frac{N_{\text {Trig }}}{N_{\text {Muons }}}
$$



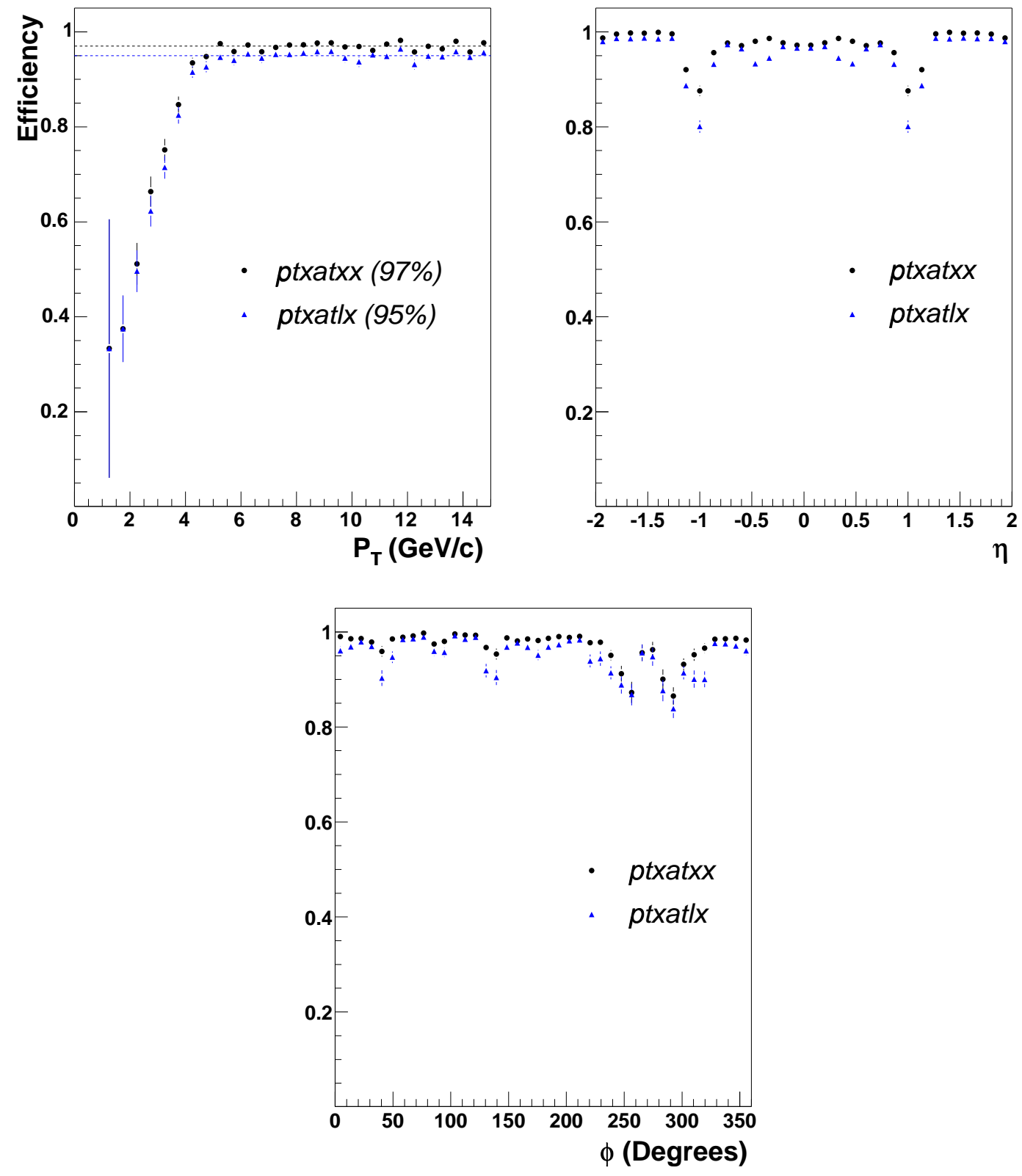

Figure 4.7. The Monte Carlo efficiency for the tight scintillator only and tight scintillator/loose wire trigger. 
where $N_{\text {Muons }}$ is the total number of events with a medium muon passing the above cuts and $N_{\text {Trig }}$ is the total number of events with a medium muon and a trigger. Figure 4.8 shows the efficiency for the mulptxatxx and mulptxatlx triggers from data. The fit for the $\mathrm{P}_{\mathrm{T}}$ distribution and the $\eta$ and $\phi$ distributions are for muons with $P_{T}>11 \mathrm{GeV} / \mathrm{c}$. 

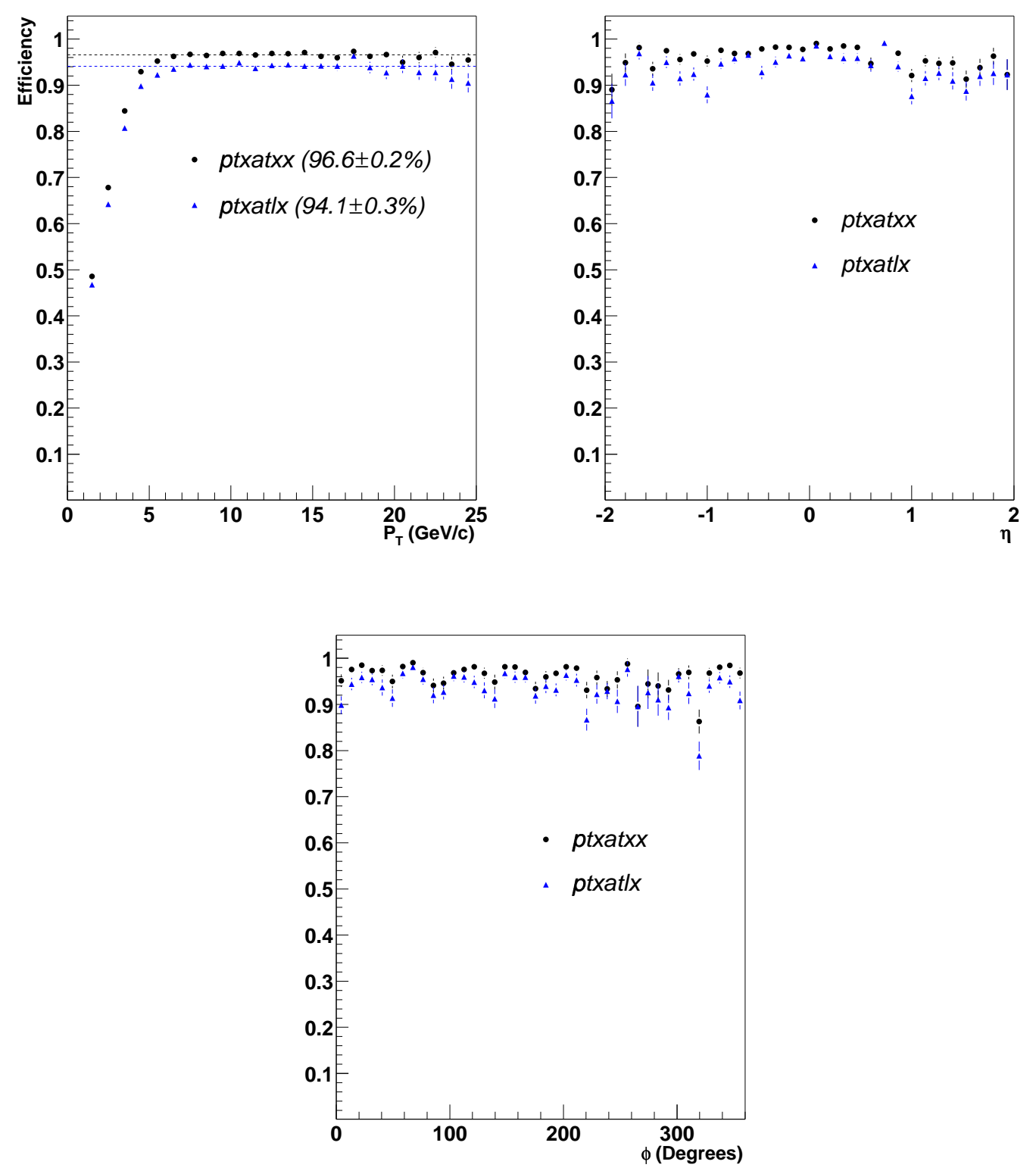

Figure 4.8. The efficiency for the tight scintillator only and tight scintillator/loose wire trigger from data. 


\section{CHAPTER 5}

\section{LeVel 1 Muon Operations}

Control of the roughly 750 serial links, 50 trigger cards, and readout of the L1MU trigger is handled largely with control registers on the trigger cards. Diagnostic information concerning bad serial links, noisy channels, and other problems comes from status registers on the trigger cards and from the readout of the trigger information. This chapter describes the flow of data through the L1MU trigger system, the function of the various status and control registers, and the readout path for the trigger data.

\subsection{Data Flow}

The flow of data through the Level 1 Muon Trigger system is controlled by the data received on the individual trigger cards by the receiver daughter cards. Since data for a given event are received from the various front ends at different times, the MTCxx's must synchronize all input data before the event can be processed. Next, all trigger cards must complete their logic before a regional trigger decision can be formed on the MTCM. Finally, the regional trigger decisions from the central, north, and south crates must be synchronized at the MTM before the global triggers can be formed.

\subsubsection{Accelerator Timing}

One turn in the accelerator is defined as the time for a proton to make one revolution and is equal to $21 \mu \mathrm{s}$. Each turn in the accelerator is divided into $159132 \mathrm{~ns}$ bunch crossings (BC). Finally, every bunch crossing has seven 18.8 ns RF clock strobes.

There are three 'super bunches' of 36 bunch crossings separated by gaps of 17 crossings. One of these gaps is designated as the syn-ch gap, during which no data 
are sent to the L1MU trigger (Figure 5.1). The first crossing after Synch Gap is labeled bunch crossing 7 .

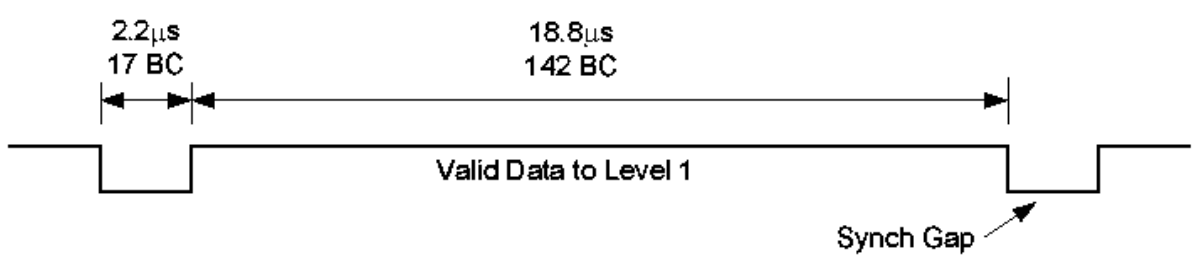

Figure 5.1. The accelerator timing for one turn. Data are sent to the L1MU trigger for the 142 bunch crossings outside of the Synch Gap. During the Synch Gap, idle characters are sent allowing the input FIFO's to go empty.

\subsubsection{Trigger Cards - Inputs}

Each trigger card (MTCxx) has sixteen serial receivers that can be selected as inputs to the trigger logic. Each receiver decodes 16 bits of data every $18.8 \mathrm{~ns}$ along with an $\mathrm{RF}$ clock that is aligned with the data. The receiver also decodes the data-available $\left(\mathrm{DAV}^{*}\right)$ signal that indicates if the decoded bits correspond to valid data or idle characters (K28.5), which are sent during the Synch Gap. The transition from idle characters to valid data marks the beginning of data for BC 7 .

The received data are first written into FIFO's that buffer inputs that arrive early while waiting for the other inputs. The FIFO's also handle the transitions between clock domains, since the phase of the RF clock decoded with the receiver data are completely asynchronous to the MTCxx RF clock received from the Trigger Framework via the Crate Manager. While there are seven RF clock strobes/bunch crossing, a parity error word is sent on the seventh strobe to detect transmission errors in the previous six words. Thus, each receiver can handle a total of

$$
16 \frac{\text { bits }}{\text { strobe }} \times 6 \frac{\text { strobes }}{B C}=96 \frac{\text { bits }}{B C}
$$


The empty flags on the FIFO's are initially low (active). As each FIFO receives data, the empty flag is set high. These empty flags are monitored to determine when all of the FIFO's have received data. The logical AND of the empty signals forms the input-ready signal on the MTCxx, indicating that all unmasked inputs (Section 5.3.2) have valid data. Once all FIFO's are not empty, the data are read, stored in Dual Port Memories (DPM's) for readout to Level 3, and sent to the flavor card for trigger formation. All FIFO's receive the same total number of data words (994 words/turn) and thus go empty during the Synch Gap when the front ends stop sending data.

The input-ready signal is the primary monitor for data flow into the trigger card. Each MTCxx has a NIM output of this signal, and each MTCM has another that is a logical AND of the input ready from every MTCxx in the crate. By masking off all other inputs, dead or noisy channels can be examined by looking at input ready with an oscilloscope.

\subsubsection{Flavor Cards}

The flavor cards receive the synchronized data from the MTCxx motherboard and perform combinatorial logic to produce trigger decisions. In addition to the data, three timing signals are sent: RF-clock, BC-clock, and start-processing (Figure 5.2). The RF clock is the $18.8 \mathrm{~ns}$ accelerator clock and is the global clock for all logic. The BC-clock (bunch crossing clock) is high for the first of seven $18.8 \mathrm{~ns}$ RF strobes in a given bunch crossing and is used to demultiplex the data. The start-processing signal is a copy of input-ready and goes high three RF clocks before the first data are sent to the flavor card and stays high for the entire $18.8 \mu$ s of data sent to the flavor card. This signal is used by the summary FPGA on every flavor card to form the Beginning Of Turn (BOT) synchronization trigger. The data-available signal is formed by delaying start-processing by an amount equal to the trigger logic latency and is used by the MTCxx to begin reading the trigger decisions from the flavor card. 


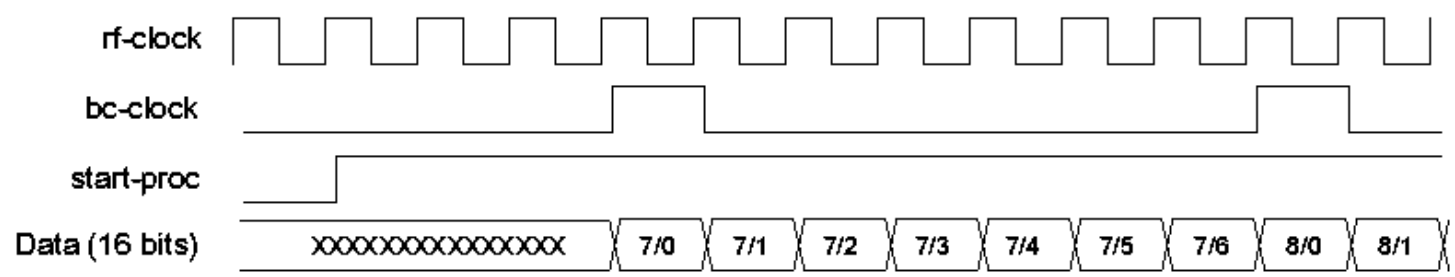

Figure 5.2. Data and timing signals sent to the flavor card. The start-processing signal is the same as input-ready and is high the entire time data are present. BCclock marks the first of seven $18.8 \mathrm{~ns}$ strobes of data to the flavor card. The data are labeled as bunch crossing/strobe.

\subsubsection{Trigger Cards - Outputs}

A total of 36 bits of octant trigger decision data from each pair of MTCxx cards is sent to the MTCM. The 36 bits are multiplexed in 12 bits $\mathrm{x} 3$ strobes across the backplane. Each MTCxx card must read the trigger decisions from the flavor card, multiplex the data, and send the data to the MTCM. Additionally, since a pair of MTCxx's share the 12 bits of backplane signals, each MTCxx must send data at the correct time.

The data-available signal from the flavor card indicates that the trigger decisions for BC 7 have been formed and can be latched into the data-multiplexer chip. The data-multiplexer reads the 36 bits of trigger decision from the flavor card and divides it into three groups of 12 bits each for transfer across the backplane. When the trigger decision data have been multiplexed, the results are written into the trigger decision FIFO on the MTCxx in a 2-2-3 RF clock structure (Figure 5.3), with the first and second strobes written for two RF clocks and the third strobe for three.

When the empty flag from the trigger decision FIFO goes not empty, the MTCxx raises data-ready, indicating it has formed the triggers for the first event and these can be read by the Crate Manager. When data-ready goes active on the MTCxx, it also raises a shared data-ready signal on the backplane. When all MTCxx's have 


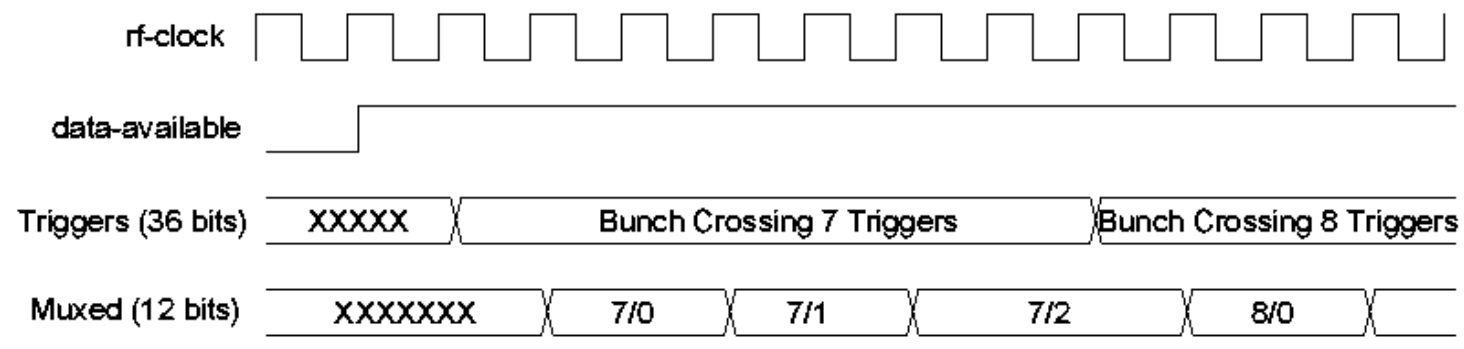

Figure 5.3. Timing of the trigger decisions from the flavor card. The 36 bits of trigger information for BC 7 are sent from the flavor card and are divided into three groups of 12 bits. The multiplexed trigger decisions are read by the MTCM across the backplane and summed for the eight octants.

raised this signal, the MTCM sees data-ready go high and can begin reading the data stored in the trigger decision FIFO's. In this way, the trigger decision FIFO's on the MTCxx's synchronize the data for different trigger cards just as the input FIFO's synchronize the various FE data for a given MTCxx.

The sharing of the backplane signals between the MTC05 and the MTC10 is handled by the nibble control register on the data multiplexer chip. For each trigger card in an octant, the 36 bits are divided into nine 4-bit nibbles. The nibbles that should be sent to the MTCM are enabled on each MTCxx via a register (Section $5.3 .2)$.

\subsubsection{Crate Manager Logic}

When the MTCM sees data-ready go high, it responds with the send-data signal, which starts the readout of the trigger decision FIFO's on the MTCxx's. The data from each octant are sent across the backplane and written into Dual Port Memories (DPM's) on the MTCM for buffering awaiting the Level 1 and Level 2 trigger decisions from the Trigger Framework. The data are also latched on the test-data-mux chip and then sent to the MTCM-logic chip, where the regional trigger logic is performed. 
The regional trigger logic on the MTCM first demultiplexes the triggers from the eight octants. It then sums the various two-bit counters from the octants to report a regional value of 0,1 , or 2 for each trigger (presently, there is no count to 3 trigger logic). It also combines the MTC05 and MTC10 triggers to form the combination triggers, e.g., a tight scintillator and loose wire trigger in a given octant. The regional logic can also mask off individual octants (Section 5.3.1) from the regional trigger decisions.

The output of the MTCM logic is a total of 72 bits - 36 bits are the raw trigger sums from the eight octants and 36 are the combined 05/10 triggers. As with the individual octant trigger decisions, these take the form of 2-bit counters. These are multiplexed into 12 bits x 6 strobes and sent to the MTM-message chip, which formats the data for the serial transmitter. While the current multiplexing scheme maintains the structure of the 12-bit backplane multiplexing, it could be increase to 96 bits by using 16 bits x 6 strobes. The MTM-message chip delays the send-data signal, aligns it with the first regional trigger decisions, and sends it to the serial transmitter as transmit enable.

\subsubsection{Trigger Manager Logic}

The trigger manager is a flavor card mounted on an MTCxx, just like the MTC05 and MTC10 flavor cards. It uses the same input buffering scheme described in Section 5.1.2 to buffer and synchronize the regional trigger decisions from the central, north, and south MTCM's. The regional decisions are combined on the MTM to form 256 global trigger terms, up to 32 of which can be sent to the Trigger Framework.

Triggers are sent to the Trigger Framework on two 40c twist and flat cables. Each cable carries 16 bits of global trigger decisions and two timing signals, strobe and gap. The strobe signal is a $132 \mathrm{~ns}$ clock that is used by the Trigger Framework (TF) to clock in the trigger decisions. The gap signal is high during the bunch crossings 
in the Synch Gap and is used by the TF to determine which crossing corresponds to BC 7, the first real bunch crossing of each turn.

\subsubsection{Trigger Framework}

The Trigger Framework expects the trigger decisions from the Level 1 systems within $3.35 \mu$ s of the actual crossing. The trigger decisions arriving from the Level 1 systems are stored in FIFO's at the TF and readout a fixed time after the crossing occurred. During an SCL init, these FIFO's are reset. After the SCL init has been dropped, the TF continues to reset these FIFO's for roughly one second, allowing the Level 1 systems time to stabilize the trigger inputs to the TF.

When the $\mathrm{TF}$ is ready to begin reading trigger decisions, it uses the falling edge of the gap signal to mark the triggers for $\mathrm{BC} 7$ and begins writing the words into a FIFO using the strobe signal as a clock. Since the TF waits a set amount of time after the collision to begin reading the trigger decisions from the FIFO's, the number of words in the FIFO at a given time is an indication of how early the triggers arrived at the TF. This number is displayed on the DAQ Monitor GUI in the FIFO column and is typically 1 for the L1MU trigger, meaning the L1MU triggers arrive at the TF about $132 \mathrm{~ns}$ before the maximum allowed delay time. A value of 255 always indicates a problem with the synchronization and usually means that one or more inputs are dead.

\subsection{Readout}

All trigger decisions formed on the trigger cards are buffered pending readout to Level 2 and Level 3. Upon a Level 1 Accept, the three regional crates send all octant triggers to the Level 2 muon trigger system. In addition, the Trigger Manager sends the 32 bits of global trigger information. 
Upon a Level 2 Accept, the trigger crates send all readout information to the Level 3 trigger for inclusion in the event stream. This includes all octant triggers and crate and card error flags. Optionally, a long message can be sent that adds trigger card information (firmware version, mask register settings, etc.) and all input data received by the MTCxx's. If the event is accepted at Level 3, this information is stored to tape as part of the event for offline analysis.

All readout and trigger timing and control signals originate with the Trigger Framework, located in MCH1. The TF receives the accelerator clock and passes it to all parts of the experiment via the Serial Command Link (SCL) along with Level 1 and Level 2 trigger decisions. In the muon system, the SCL signals are received by the Muon Fanout Card (MFC) that resides in a VME crate in MCH3. The fanout card passes these signals to multiple Muon Readout Cards (MRC) that send them to the individual front end systems (Figure 5.4).

The readout crate that holds the fanout and readout cards also has the first stage of the Level 3 system, the Single Board Computer (SBC). The SBC reads the data for a given event from all MRC's in the crate and sends it via Ethernet through two switches to the various Level 3 farm nodes that handle the Level 3 filtering. The VME master is a PowerPC processor that coordinates the readout of the data and also provides monitoring and diagnostic information for the readout crate.

\subsubsection{Message Builder}

When the MTCM receives a Level 1 or Level 2 Accept from the Trigger Framework, the Message Builder FPGA begins reading a list of commands from memory that indicate what data to send. The commands typically point to various memory locations on the MTCM and the MTCxx's that have buffered data for a given event. For each command, the Message Builder reads the appropriate memory location and sends the data to the MRC via Hotlink serial communication. Since the content of 


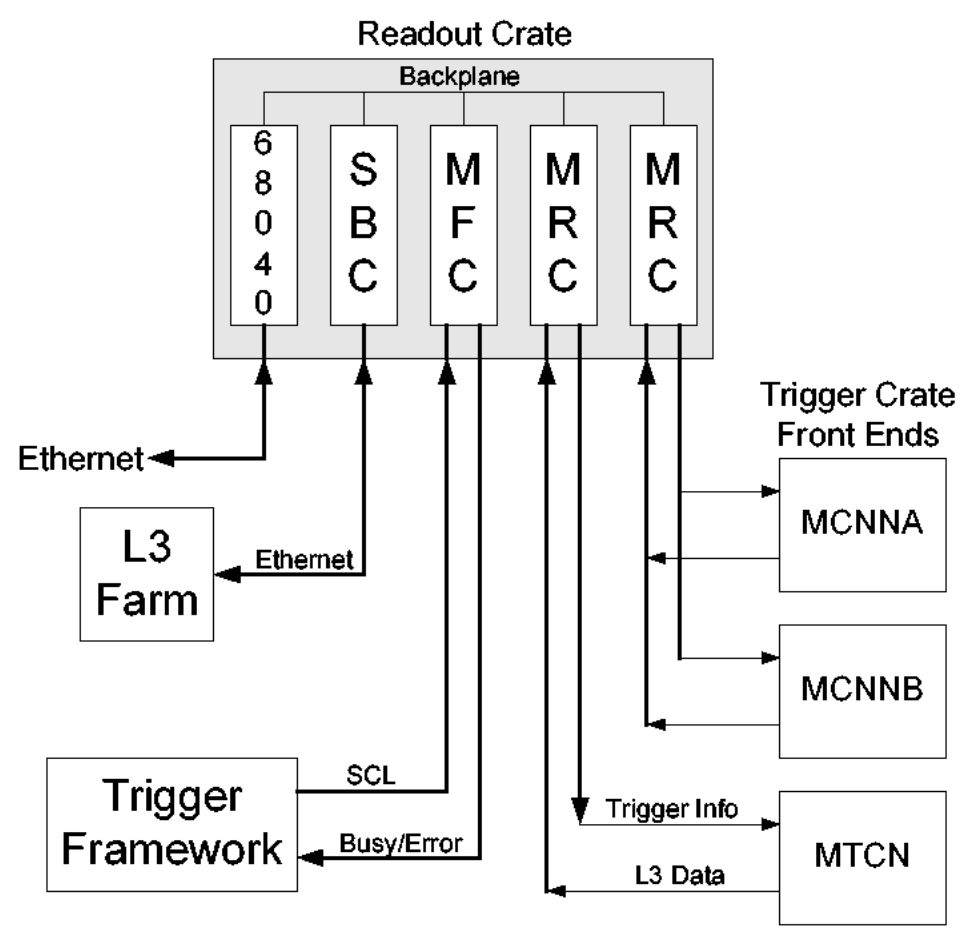

Figure 5.4. A schematic view of the readout path for the north trigger crates. All trigger information originates with the Trigger Framework and is distributed to the Geographic Sections over the Serial Command Link (SCL). Upon receiving a Level 2 Accept, the front ends send data to the Muon Readout Cards, which are then readout by the Single Board Computer and sent to the Level 3 farm nodes. 


\begin{tabular}{|l|l|c|c|}
\hline L1MU Front End & Description & Readout Crate & MRC Section \\
\hline MCNNA & North A MCEN Crate & L1MTN (0x17) & $2 \mathrm{~A}$ \\
\hline MCNNB & North B/C MCEN Crate & L1MTN $(0 \times 17)$ & $2 \mathrm{~B}$ \\
\hline MCNSA & South A MCEN Crate & L1MTS $(0 \times 18)$ & $2 \mathrm{~A}$ \\
\hline MCNSB & South B/C MCEN Crate & L1MTS $(0 \times 18)$ & $2 \mathrm{~B}$ \\
\hline MCNC & Central Concentrator Crate & L1MTC $(0 \times 16)$ & $0 \mathrm{~B}$ \\
\hline MTCC & Central Trigger Crate & L1MTC $(0 \times 16)$ & $0 \mathrm{~A}$ \\
\hline MTCN & North Trigger Crate & L1MTN $(0 \times 17)$ & $0 \mathrm{~A}$ \\
\hline MTCS & South Trigger Crate & L1MTS $(0 \times 18)$ & $0 \mathrm{~A}$ \\
\hline MTM & Muon Trigger Manager & L1MTM $(0 \times 19)$ & $0 \mathrm{~A}$ \\
\hline
\end{tabular}

TABLE 5.1. Map of the nine L1MU trigger front end crates to the readout crate and section.

the message is stored by the user in memory, the message is easy to change.

\subsubsection{Muon Readout Crate}

The muon readout crate is responsible for receiving the timing and control signals from the Trigger Framework and passing it to the front end systems on the platform. It also acts as the interface between a collection of front ends (called a Geographic Section) and Level 3. For the L1MU trigger, there are four geographic sections (and thus readout crates): Central (L1MTC), North (L1MTN), South (L1MTS), and the Muon Trigger Manager (L1MTM). The centroid finding crates, which form track stubs for the forward wire chambers and concentrate scintillator inputs, are also readout in the central, north, and south regions. Table 5.1 shows the mapping of the nine L1MU front end crates to the readout crate and section.

The Level 3 data for a given event are received by the MRC's. Each MRC has two independent sections, A and B, that handle different front ends. Once all MRC sections indicate they have all of the data from the various front ends for a given event, the SBC begins reading the data from the MRC's and sending it to the Level 3 farm nodes. 


\begin{tabular}{|l|l|l|l|}
\hline Name & \multicolumn{1}{|c|}{ Address } & Value & Location \\
\hline Bus Grant Control & 0x1040002c & 0x6 & VME Interface \\
\hline L2 Ratio & 0x10400030 & 0x0 & Message Builder \\
\hline L3 Ratio & 0x10400032 & 0x8000 & Message Builder \\
\hline Buffer Depth & 0x1040002a & 0x40 & DPM-FIFO \\
\hline First BC & 0x10400006 & 0x7 & Timing Generator \\
\hline Data Ready Length & 0x10400046 & 0x8e & Timing Generator \\
\hline L1 Reject Begin & 0x10400042 & 0x7 & DPM-FIFO \\
\hline L1 Reject Length & 0x10400048 & 0x8e & DPM-FIFO \\
\hline Trigger Enable & 0x10400020 & 0xffff & Trigger Logic \\
\hline Readout Enable & 0x1040001e & 0xffff & Message Builder \\
\hline Error Mask & 0x10400002 & 0x0 & Timing Generator \\
\hline Timing Mode & 0x10400080 & 0x1 & Timing Generator \\
\hline Meta Protect & 0x10400082 & 0x1 - 0x7 & Timing Generator \\
\hline BC Gain & 0x10400086 & 0x2 & Timing Generator \\
\hline
\end{tabular}

TABLE 5.2. A summary of the registers set on the MTCM, the values to which they are set, and the CPLD that implements the register.

\subsection{Control Registers}

Much of the operation of the trigger crates is configured via control registers on the Crate Managers (MTCM's) and the individual trigger cards (MTCxx's). These registers are set in text files and are loaded to the crates upon a cold start or a crate restore.

\subsubsection{MTCM}

The MTCM performs a variety of functions, including the formation of regional trigger decisions, receiving the timing and control signals from the MRC, and sending trigger information to Level 2 and Level 3. A summary of the registers and their settings is given in Table 5.2. The hardware specification for the MTCM is here [37]. A detailed description of the various registers is in the following sections. 


\begin{tabular}{|l|c|l|}
\hline Section & Bit & Purpose \\
\hline 1553 Interface & 0x2 & Allow 1553 VME bus requests \\
\hline Message Builder & 0x4 & Allow Message Builder bus requests \\
\hline FPGA Config & 0x8 & Allow FPGA Config bus requests \\
\hline
\end{tabular}

TABLE 5.3. Bus grant control for the MTCM. Setting a given bit high to this register will enable the given section access to the VME bus.

\section{Bus Grant Control (0x1040002c)}

The MTCM is the only card in the crate that can request the bus from the MVME162 processor. There are three independent sections on the MTCM that can request the bus - the Message Builder, the 1553 interface, and the FPGA programmer. Table 5.3 shows the control bits for allowing these sections control of the VME bus.

In normal operation, the 1553 and Message Builder are allowed to request the bus. The FPGA programmer only needs the bus when it is configuring the MTCM logic and the Message Builder during a cold start, which is handled in the startup software.

\section{Message Ratio (0x10400030, 0x10400032)}

Upon receiving a Level 1 Accept, the MTCM sends trigger information for the event to Level 2 (L2 Message). Upon receiving a Level 2 Accept, the crate sends all readout information to Level 3 (L3 Message). Two different messages (short/long) can be sent upon a Level 1 or Level 2 Accept. In practice, the L2 message is fixed, while the long L3 message includes all of the input data to the trigger cards.

A value of $0 x 0$ for this register will always send the long message, while 0x8000 will always send the short message. Any other value sets the number of short messages sent for every one long. A setting of 0x5 will send one long, five short, etc.

\section{Buffer Depth (0x1040002a)}


The total number of available buffers used to hold data pending readout to Level 2 and Level 3 is set by this register. The data must be buffered pending the Level 1 decision for a given event. The total number required is variable and depends on the latency, but the worst case is about 33 bunch crossings, which corresponds to the time between the bunch crossing occurring and the trigger decisions for that crossing arriving at the crate. There must also be enough buffers to store up to 16 Level 1 Accepts pending the Level 2 decision and up to 8 Level 2 Accepts pending readout to Level 3. This register is set to 64 (x40), and has a maximum value of 255 (0xff).

\section{First BC (0x10400006), Meta Protect (0x10400042), and Data Ready Length (0x10400046)}

The current data structure is 142 crossings of data followed by 17 crossing in the Synch Gap during which all front ends send idle characters to the L1MU trigger (Section 5.1.1). After an SCL init, the Timing Generator on the MTCM receives the data-ready signal from the sixteen MTCxx cards, indicating that they have all produced triggers for the first bunch crossing. The timing generator responds with the send-data signal, which is used to read the trigger decisions on the MTCxx's and send them to the MTCM.

When the first data-ready is received from the MTCxx's after an SCL init, it is passed through a variable-length shift register, whose length is set by the Meta Protect register $(0 x 10400082$, values 0x0 - 0x7). In theory, this register should only protect against meta-stability problems due to the slow rise time of data-ready on the backplane. In practice, it is sometimes necessary to change this register to get the Crate Manager to readout in synch. After passing through the shift register, dataready is used to set the local crossing number to the value of the First BC register at 0x10400006 (0x7) and the local turn number to 0x1. These values are written into 
the DPM's along with the trigger decisions from the MTCxx's and are readout to Level 3 as a check of synchronization. These are the values of the turn and crossing numbers that are displayed on the muon readout GUI.

Because of the slow rise time of data-ready on the backplane, it is not used to synchronize every turn. Rather, the timing generator looks for the first data-ready after an init and counts all subsequent data-ready signals from there. In addition to generating the send-data signal, data-ready is used by the DPM-FIFO-Control CPLD to correctly buffer the trigger data and turn and crossing numbers. The total length of data-ready is set by the Data Ready Length register (0x10400046) and is set to $142(0 \mathrm{x} 8 \mathrm{e})$.

\section{BC Gain (0x10400086)}

On the first turn after an SCL init, the data are stored in the trigger decision FIFO's on the MTCxx cards while the MTCM responds to the data-ready signal and generates the send-data signal. This delay is about two full bunch crossings in length (256 ns) and adds significant latency to the L1MU trigger. However, because the data-ready signal is 'counted' on the MTCM for all turns after the first, the arrival of the trigger data at the MTCxx's can be anticipated and the send-data signal can be sent earlier.

This has been implemented with a register in the Timing Generator called the BC Gain. The value of the BC Gain register (0x10400086) is set to the number of $132 \mathrm{~ns}$ bunch crossings to skip during the first Synch Gap and is currently set to 0x2. This removes most of the latency caused by the rise time of data-ready on the backplane, the delay of data-ready through the shift register, and the setup time required to generate the send-data signal. While this register steps in increments of $132 \mathrm{~ns}$, it can be combined with the MetaProtect register, which delays the initial data-ready, to tune the arrival of send-data at the MTCxx's to any increment of $18.8 \mathrm{~ns}$. 


\section{L1 Reject Begin (0x10400042) and L1 Reject Length (0x10400048)}

The trigger decisions sent from the Trigger Framework to the MTCM include Level 1 Accept, L2 Accept, and L2 Reject. The framework also sends an 8-bit counter indicating the bunch crossing number for each trigger. The Level 1 Reject signal is formed by the lack of a Level 1 Accept for bunch crossings that have data.

The MTCM will begin generating Level 1 triggers when the BC counter sent by the framework is equal to the value of the L1 Reject Begin register (0x10400042), which is set to $0 x 7$. The MTCM will stop generating Level 1 triggers based on the L1 Reject Length register (0x10400048). Setting this to 142 (0x8e) generates 143 Level 1 triggers/turn. This is one more than the number of crossings due to the buffering on the MTCxx (Section 5.3.2).

\section{Trigger Enable (0x10400020)}

The MTCM reads the octant trigger decisions for all eight pairs of MTCxx cards and combines these into a regional sum. These regional sums include triggers formed from the MTC05 or MTC10 cards alone ('raw' triggers) and triggers that are combinations of the two cards for a given octant ('combined' triggers). The octant trigger decisions can be masked out of the trigger using this register. Table 5.4 gives the mapping for the octants. A mask of 0xffff enables all triggers for all octants, 0x5555 just enables the raw triggers for all octants, and 0x1 would simply look at the raw triggers for octant 0 . This mask is used primarily for octant rate studies and isolating hot inputs. Also, if all inputs to the MTC05 and MTC10 in an octant are masked off, this octant must be masked off in this register as well, as the backplane lines for that octant will float and are not held low. 


\begin{tabular}{|l|l|l|}
\hline Bit & Octant & Purpose \\
\hline 0 & 0 & Enable raw 05 and 10 triggers \\
\hline 1 & 0 & Enable combined $05 / 10$ triggers \\
\hline 2 & 1 & Enable raw 05 and 10 triggers \\
\hline 3 & 1 & Enable combined $05 / 10$ triggers \\
\hline$\ldots$ & $\ldots$ & $\ldots$ \\
\hline 14 & 7 & Enable raw 05 and 10 triggers \\
\hline 15 & 7 & Enable combined $05 / 10$ triggers \\
\hline
\end{tabular}

TABLE 5.4. MTCM trigger logic settings used to enable octant triggers. Setting a bit high will include a given octant in the trigger.

\section{Readout Enable (0x1040001e)}

Upon receiving a Level 1 or Level 2 Accept, the Message Builder reads from a script of commands that are stored in NVRAM on the MTCM. To avoid having to change this script when an MTCxx is removed from the crate, an enable register is set to tell the Message Builder what cards can be read. When it encounters a command to read from a given MTCxx, it checks that the bit corresponding to that MTCxx is set in the enable register. If the bit is low, it simply sends zeros as a substitute for the words from that MTCxx and does not attempt to read from that card.

\subsubsection{MTCxx}

The masks on the MTCxx's are concerned mainly with the control of the data from the sixteen receivers. Table 5.5 summarizes these registers and their location. The hardware specification for the MTCxx is here [36].

\section{Mask Registers}

The data are received on the MTCxx's by sixteen serial receiver cards. There are four registers that control various masks. Each register works by setting the bit 


\begin{tabular}{|l|l|l|l|}
\hline Name & \multicolumn{1}{|c|}{ Address } & Value & Location \\
\hline Serial Mask & 0x2n000006 & 0x0 - 0xffff & RCV-FIFO-Control \\
\hline Trigger Mask & 0x2n000008 & 0x0 - 0xffff & Data-mux \\
\hline Parity Mask & 0x2n00000a & 0x0 - 0xffff & RCV-FIFO-Control \\
\hline Lock Mask & 0x2n00000c & 0x0 - 0xffff & RCV-FIFO-Control \\
\hline Nibble Control & 0x2n00000e & 0x0 - 0x1ff & Data Multiplexer \\
\hline Meta Protect & 0x2n000096 & 0x0 - 0x3 & RCV-FIFO-Control \\
\hline First BC & 0x2n00001e & 0x7 & DPM-FIFO-Control \\
\hline Last BC & 0x2n00001c & 0x95 & DPM-FIFO-Control \\
\hline Auxiliary Control & 0x2n00001a & 0x0 & RCV-FIFO-Control \\
\hline Test & 0x2n000022 & 0x0 & Data-mux \\
\hline
\end{tabular}

TABLE 5.5. MTCxx control masks.

high that corresponds to the receiver that should be masked. For example, a mask of 0x0001 masks off receiver 0, 0x0002 masks off receiver 1, and a mask of 0xffff masks off all receivers. The four separate masks are:

Serial Mask (0x06): Determines which FIFO's are polled to form input-ready. Input ready goes active when all unmasked FIFO's are not empty, and goes inactive when any unmasked FIFO is empty. When input ready is active, the data are read from all FIFO's and sent to the flavor cards to for the trigger decisions.

Trigger Mask (0x08): Once input ready has gone active, the data from all FIFO's are read and stored in memory. To keep certain inputs from contributing to the trigger decisions on the flavor card (e.g., to mask out a problem receiver channel), this register can be set high for that receiver.

Parity Mask (0x0a): The MTCxx forms a parity error bit if any of receivers not masked by this register detect a parity error.

Lock Mask (0x0c): The MTCxx forms a lock error bit if any of the receivers not masked by this register detect a lock error.

In normal operation, all of these are set to the same value. One useful exception would be to include a receiver in the serial mask (that is, force the card to wait for this input to have data) but mask it off with the trigger mask. This would allow 
readout of the input in the data, but would not affect the trigger rates, and would be useful in debugging a problematic channel.

Finally, the serial mask register changes (in theory) the overall timing of the inputs and requires an init after any changes are made to re-synch the system. The trigger mask register does not affect the timing of the data flow, and thus does not need an init after changes are made.

\section{Nibble control (0x2n00000e)}

As described in Section 5.1.4, the MTC05 and MTC10 for a given octant must share 12 bits on the backplane to send triggers to the MTCM. Both trigger cards produce 36 bits of trigger decision, which are divided into nine nibbles. Setting a given bit high on a trigger card will enable that nibble to be sent to the MTCM.

Currently, for example, the central region enables the low seven nibbles for the MTC05 cards (0x7f) and the upper two nibbles to the MTC10 (0x180). An 05/10 pair should never try to enable the same nibble.

\section{Meta Protect (0x2n000096)}

The FIFO empty flags are monitored and compared against the serial input mask. When all of the unmasked FIFO's are not empty, the data are ready to be read and sent to DPM's and to the flavor card. Since the non-empty signal from the FIFO's is asynchronous to the card RF clock, it is passed through a number of flip-flops similar to data-ready on the MTCM. This register is also used to get the MTCxx to stay in synch, again analogous to the MTCM.

\section{First/Last BC (0x2n00001e, 0x2n00001c)}


When the DPM-FIFO control receives the start-processing signal, it sets the value of the bunch crossing counter to the value in register 0x2n00001e, which is set to 7 . This is the BC for the first data, and is written into DPM with the first data. Register 0x2n00001c sets the last crossing for which data are stored in the DPM and is set to 149. A total of 143 crossings of data are written into the DPM's due to an old feature of the logic that used the end of data to stop writing into the DPM's. Since the MTCxx could not respond immediately to the end of data, it would write an extra BC's worth of data. This is compensated for on the MTCM by sending one extra Level 1 Reject for every turn.

\section{Auxiliary Control and Status (0x2n00001a)}

The auxiliary control register allows data-ready to be forced high regardless of the state of data-available from the flavor card, if for example the flavor card failed to configure. It also allows the reset of the receivers on SCL init to be disabled. In normal operation, these two bits are set low.

There are also three bits of status information on this register. The latched FIFO empty flag checks if any of the unmasked FIFO's did not go empty during the Synch Gap. In normal running, all FIFO's should go empty at the same time. The latched FIFO lock error and FIFO full error indicates that one of the unmasked FIFO's showed a lock error or a FIFO full flag since the last clear. Bits 2-4 are reset by writing a 0 to each bit.

\section{Test Mode (0x2n000022)}

The test mode is used to send user-defined patterns from the DPM to the flavor card. In normal operation, this register is always set to $0 \mathrm{x} 0$. 


\begin{tabular}{|l|l|}
\hline Bit & Purpose \\
\hline 0 & Force data-ready high \\
\hline 1 & Disable reset of receivers on init \\
\hline 2 & Latched FIFO empty error \\
\hline 3 & Latched lock error \\
\hline 4 & Latched FIFO full error \\
\hline
\end{tabular}

TABLE 5.6. Auxiliary control and status register.

\begin{tabular}{|l|l|l|}
\hline Register & Address & Description \\
\hline Lock & 0x2n000088 & High indicates bad lock (Real-time) \\
\hline FIFO full & 0x2n00008c & High indicates FIFO full (Real-time) \\
\hline Parity & 0x2n000092 & High indicates bad parity (Latched) \\
\hline
\end{tabular}

TABLE 5.7. Serial link status registers. Each register has 16 bits, one for each serial link on the MTCxx.

\subsection{Status Registers}

Diagnostic information from every trigger card comes from the status registers. Most issues with data flow stem from problems with one or more of the receivers. To detect these problems, each serial link has three registers to detect error conditions: lock error, parity error, and FIFO full. Table 5.7 gives the addresses for these registers.

A lock error is generated by the receiver and is usually caused by an unplugged cable or a front end that has been powered down. A link that is showing bad lock will almost certainly stop data flow through the system. Parity errors are also generated on the receivers and indicate that there was a transmission error in the previous bunch crossing. Since the parity errors for a given crossing are only high for $132 \mathrm{~ns}$, this register is latched. This means that any parity error since the last clear will set the bit high and keep it high until the next clear. This register is cleared by writing a 0 to it. Finally, the FIFO full error is generated from the full flag of the input FIFO's. In normal running, the FIFO's should never be full. A single full FIFO usually indicates a bad cable that is causing the receiver to decode too much data 
and should be accompanied by a parity error. Many full FIFO's usually indicate that data have stopped coming from the receivers whose FIFO's are not full. When data are not sent to a given receiver, it holds up the reading of all FIFO's on that MTCxx, which will cause all other FIFO's to go full.

The FIFO full and lock errors also have a latched register (Table 5.6). This register is a single bit and indicates that one of the unmasked links had a FIFO full or lock error.

\subsection{Trouble Shooting}

\subsubsection{Trigger Rates}

Most problems with trigger rates are due to problems in one or more of the front ends. If there are problems with the trigger rates, the first course of action should be to check that all FE's are in global mode with no disabled channels.

In general, all muon readout crates should be in global running mode, which means the readout client will display 'No Data Flow' or 'Running' in the status bar. Any front-ends that are removed from the muon readout should be removed from the L1MU trigger. When a FE is removed from the muon readout, the readout client will display the 'Check Configuration' message.

The L1MU trigger also gets inputs from L1CTT. For these inputs to be good, Geographic Sections 0x50 (axial CFT) and 0x52 (Central Preshower) must be in global data taking mode (not calibration mode). If crates 0x50 and 0x52 are in the

global run, they are in global mode. The synch gap length can be checked on the DFEA's for all 80 sectors with the dfe_ware GUI that is run by the CFT shifter.

- BOT Rates are 0: Check for dead inputs. Any dead input can cause all rates to be zero. Look at the FIFO-Full display on the monitor GUI - a dead input will show up as the only unmasked FIFO that is not full on a given trigger card. 
It is possible that the bad input will also show up on the parity error register as well.

- BOT Rates are non-zero but not 47712: This is usually the case when one or more FE's are doing calibrations or other work. This can also be caused by a bad cable that is causing data flow problems on an MTCxx. Check the parity register for all cards to look for a bad input.

- A particular trigger rate is high: This often is the signal that a cable is bad and can be seen by checking the parity error register. This can also be caused by a problem on a front end. Check the trigsim comparison plots to see if any of the octants are showing large positive differences (i.e., hardware but no simulator trigger). Also check the muon examine for spikes in the muon hit distributions.

\subsubsection{Readout}

The muon readout client provides control of the readout for the front end crates connected to a given readout crate. It also provides status and error information for each front end, including a synchronization check of the turn and crossing number for every event. Three counters show the number of Level 2 Accepts, Service Requests (SRQ's - the number of times the FE's sent an event), and the number of SBC Done's received, which indicate the SBC has finished reading an event. During normal operation, these three counters are the same.

Errors in the muon readout client often point to problems with a front end section. Placing the mouse over the error messages will raise a bubble window with more information.

- Readout shows error when starting: When the 'Start Readout' button is pressed, the readout crate goes through several initialization procedures. The 
most important from the front end point of view is the Hotlink receiver on the readout card is reframed. This means that the front end must be sending idle characters. A 'Hotlink Reframe Error' means that either the trigger crate is powered down, not cold-started, or the gray astro cable is not plugged in.

- Readout shows error on SCL Init: When 'Start Readout' is successful, the readout client will show 'Waiting for Init'. If the crate then shows a TFW Init Error Message, it is likely a 'Busy 1 Set Timeout'. This means that the FE did not respond with a Level 1 busy signal when the SCL init was sent and usually means that the 50c twist and flat cable is not plugged in to the FE or the back of the readout crate.

- Crate is reading out of synch: This is usually caused by one or more bad inputs. The crates calculate the synchronization based on data-ready, so a bad input will typically throw off the readout. In this case, the BOT's should also be bad. If the BOT's are OK, try adjusting the MetaProtect register (0x10400082) and send an SCL init. This can be the case especially if some inputs were recently masked off.

- Crate is raising Level 2 Busy (B2): The FE's raise L2 busy when they have buffered 8 L2 Accepts awaiting readout. This can occur naturally at high Level 2 Accept rates, but typically this occurs at a small level (few percent) as viewed on the DAQ monitor. Problems with the readout crate, such as a software crash, cause the FE to raise B2 as soon as 8 trigger have been sent. If the readout crate does not reset the counters on an SCL init, stop and restart the readout.

Another possible problem is that the connection between the SBC and Level 3 has been lost. In this case, the crate is usually raising B2 almost all of the time, the rate of Level 2 Accepts is about $1 \mathrm{~Hz}$, and events show up as missing at 
Level 3. The DAQ shifter notices missing events and can try reseting the SBC. After an SBC is reset, the readout must be stopped and restarted. 


\section{CHAPTER 6}

\section{Data Selection}

\subsection{Data Collection and Processing}

The data used in this analysis were recorded by the D $\varnothing$ detector between July, 2002 and September, 2003, and include runs 160585 to 180956. A total of roughly 480 million events were collected during this period, and all data were reconstructed with the p14 production version of the offline reconstruction program. After reconstruction, data skims were made by the Common Samples Group to reduce the data to a level manageable for analysis [41]. The expected signature in the dector for $t \bar{t} \rightarrow \mu \mu$ events is two high $\mathrm{P}_{\mathrm{T}}$ muons, two jets, and missing transverse energy. This analysis used the $2 \mathrm{MU}$ skim, which includes all events with at least two loose muons reconstructed in the muon system. The 2MU skim had a total of about 340,000 events.

The top_analyze program was used to convert the output of the reconstruction to a Root tree suitable for analysis [42]. In addition, top_analyze applied jet energy scale corrections to the jets in the event and imposed basic jet quality cuts (Section 6.5). Medium muons were identified with the certified p14 MuoCandidate package [40]. Cuts were also applied to reject cosmic ray muons in data. In Monte Carlo events, the $\mathrm{P}_{\mathrm{T}}$ of the muons was smeared to more accurately reflect the resolution of the detector. The data and all Monte Carlo samples used the Nefertiti version of top_analyze.

The top group data quality package (top_dq) was used to remove runs that had been marked bad by the muon, calorimeter, or tracking detector groups. Runs that did not pass the jet energy scale criteria were also excluded [43]. These criteria include:

1. Readout of all calorimeter crates. 


\begin{tabular}{|c|c|}
\hline Trigger List Version & Integrated Luminosity $\left(\mathrm{pb}^{-1}\right)$ \\
\hline $\mathrm{v} 8 . \mathrm{x}$ & 22.53 \\
\hline $\mathrm{v} 9 . \mathrm{x}$ & 18.59 \\
\hline $\mathrm{v} 10 . \mathrm{x}$ & 8.19 \\
\hline $\mathrm{v} 11 . \mathrm{x}$ & 55.09 \\
\hline $\mathrm{v} 12 . \mathrm{x}$ & 35.18 \\
\hline Total & 139.58 \\
\hline
\end{tabular}

TABLE 6.1. The integrated luminosity for the five versions of the global trigger list after all run quality cuts have been applied.

2. The shift in the mean values for the $\mathrm{x}$ - and $\mathrm{y}$-components of $\mathbb{E}_{T}$ must be $<4$ GeV during the run.

3. The average $\operatorname{RMS}_{X Y} \equiv \sqrt{R M S\left(E_{T x}\right)^{2}+R M S\left(E_{T y}\right)^{2}}$ must be $<16 \mathrm{GeV}$ during the run.

4. The mean of the scalar transverse energy must be $>80 \mathrm{GeV}$ during the run.

Finally, individual luminosity blocks that were marked bad by the luminosity group were also rejected. Luminosity blocks are typically rejected for a given trigger when an inconsistency is found for the reported crossing and turn number or the trigger result between the various components in the online system (Level 1, Level 2, Level 3). A smaller fraction of luminosity blocks are marked bad due to corrupted information from the readout of the luminosity scalers [44]. After all quality cuts, the final data set had a total integrated luminosity of $139.58 \mathrm{pb}^{-1}$ from five different versions of the global trigger list (Table 6.1).

\subsection{Trigger}

The data span five versions of the global trigger list. The trigger 2MU_A_L2MO was used in trigger lists v8-v10. This trigger required two tight scintillator-only triggers at Level 1 (mu2ptxatxx) and one medium muon at Level 2. The tight scintillator 


\begin{tabular}{|l|l|l|l|l|}
\hline Name & Version & Level 1 & Level 2 & Level 3 \\
\hline 2MU_A_L2MO & $8.0-10.30$ & $\begin{array}{l}\text { mu2ptxatxx+ } \\
\text { minimum bias }\end{array}$ & $\begin{array}{l}1 \text { medium } \\
\text { muon }\end{array}$ & None \\
\hline 2MU_A_L2MO & 10.35 & mu2ptxatxx & $\begin{array}{l}1 \text { medium } \\
\text { muon }\end{array}$ & None \\
$\begin{array}{l}\text { OR } \\
\text { 2MU_A_L2MO_L3TRK10 }\end{array}$ & $11 . \mathrm{x}$ & mu2ptxatxx & $\begin{array}{l}1 \text { medium } \\
\text { muon }\end{array}$ & $\begin{array}{l}15 \mathrm{GeV} / \mathrm{c} \\
\text { muon }\end{array}$ \\
\hline $\begin{array}{l}\text { 2MU_A_L2MO_L3L10 } \\
\text { OR }\end{array}$ & $12 . \mathrm{x}$ & mu2ptxatxx & $\begin{array}{l}1 \text { medium } \\
\text { muon } \\
\text { track }\end{array}$ & $\begin{array}{l}10 \mathrm{GeV} / \mathrm{c} \\
\mathrm{muon}\end{array}$ \\
& & & $\begin{array}{l}\mathrm{GeV} / \mathrm{c} \\
\text { track }\end{array}$ \\
\hline
\end{tabular}

TABLE 6.2. Trigger requirements.

triggers at Level 1 are scintillator based tracks, with one hit in the A-layer and the other in the B- or C- layer. A Level 2 medium muon fits wire chamber track segments and scintillator hits inside and outside of the muon toroid. There was an additional requirement for a minimum bias trigger at Level 1 though trigger list v10.30.

In trigger list v11, a Level 3 requirement was added to keep the trigger unprescaled at high luminosities. A logical OR of two triggers was used, both of which had the same requirement at Level 1 and Level 2 as the previous trigger lists. The trigger 2MU_A_L2MO_L3L15 required a local muon with $\mathrm{P}_{\mathrm{T}}>15 \mathrm{GeV} / \mathrm{c}$ at Level 3 . 2MU_A_L2MO_L3TRK10 required a central track with $\mathrm{P}_{\mathrm{T}}>10 \mathrm{GeV} / \mathrm{c}$ at Level 3. In trigger list v12, the $\mathrm{P}_{\mathrm{T}}$ values for the local muon and central track were reduced to $10 \mathrm{GeV} / \mathrm{c}$ and $6 \mathrm{GeV} / \mathrm{c}$, respectively. Table 6.2 summarizes the trigger requirements for the data set. 


\subsection{Luminosity Measurement}

The cross section measurement requires an accurate measurement of the luminosity delivered by the accelerator. The luminosity measurement is carried out by detecting a hit coincidence in scintillator hodoscopes on both sides of the interaction region. The hodoscopes are mounted on the inner side of the end calorimeter cryostats and cover the eta range $2.7<\eta<4.4$.

The instantaneous luminosity $\mathcal{L}$ is determined by combining the coincidence rate $R$ in the luminosity hodoscopes with the observable cross section $\sigma_{\text {eff }}$ for inelastic collisions. In the low-luminosity limit, where the probability of multiple interactions on the same crossing is very low, the luminosity is simply:

$$
\mathcal{L}=\frac{R}{\sigma_{\text {eff }}}
$$

The observable cross section $\sigma_{\text {eff }}$ is the product of the total $p \bar{p}$ inelastic cross section, the acceptance of the luminosity detector for inelastic collisions, and the detector efficiency. The total $p \bar{p}$ inelastic cross section has contributions from hardcore (HC), single diffractive (SD), and double diffractive (SS) scattering. Combining the cross sections for these three processes with the detector acceptances for each and the detector efficiency gives a total observable cross section for inelastic scattering of $46.0 \pm 2.6 \mathrm{mb}[45]$.

Multiple interactions in a given bunch crossing lead to an underestimation of the luminosity since they are counted as one interaction by the luminosity detector. The average number of interactions per crossing is given by Poisson statistics as

$$
\bar{n}=\mathcal{L} \tau \sigma_{e f f}
$$

where $\tau$ is $396 \mathrm{~ns}$, the time between collisions. At an instantaneous luminosity of $3 \times 10^{31} \mathrm{~cm}^{-2} \mathrm{~s}^{-1}$ (a typical value for data taking), there are an average number of 0.55 
interactions per bunch crossing, with a $32 \%$ chance of a single interaction and a $11 \%$ chance for two or more interactions. To correct for multiple interactions, we use

$$
\frac{\mathcal{L}_{\text {real }}}{\mathcal{L}_{\text {meas }}}=\frac{\bar{n}}{1-e^{-\bar{n}}}
$$

\subsection{Offline Muon Identification}

Reconstruction of muons in the $\mathrm{D} \varnothing$ detector is done by first reconstructing individual wire chamber and scintillator hits in the three layers of the muon system. Track segments are then found in the A- and BC-layer (the B- and C-layers are treated as one layer for reconstruction) and matched to scintillator hits. Finally, a 'local' muon is found by matching segments inside (A-layer) and outside (BC-layer) of the toroid to form a track.

These local muons are then matched to tracks in the central tracker. A quality flag for a muon, called 'nseg', indicates the number of segments used in the local muon track. For this analysis, only muons with nseg $= \pm 3$ were used, indicating that the reconstructed track contains both an A- and BC-segment [40].

\subsubsection{Muon Segment and Track Finding}

Track segments in the muon system are found by looking for tracks through an individual layer of the muon wire chambers. Each layer has three or four decks of wires, and segments are found by fitting a straight line to the hits in a given layer. If a segment is found, a match to a scintillator hit is attempted. A confirming scintillator hit improves the resolution of the segment along the wire direction, particularly in the forward region where no hit information is available from the wire chambers along the wire axis (Section 3.2.5). A straight-line fit is then attempted between the B- and C- layer segments, which have no magnetic field between them to deflect the muons. 


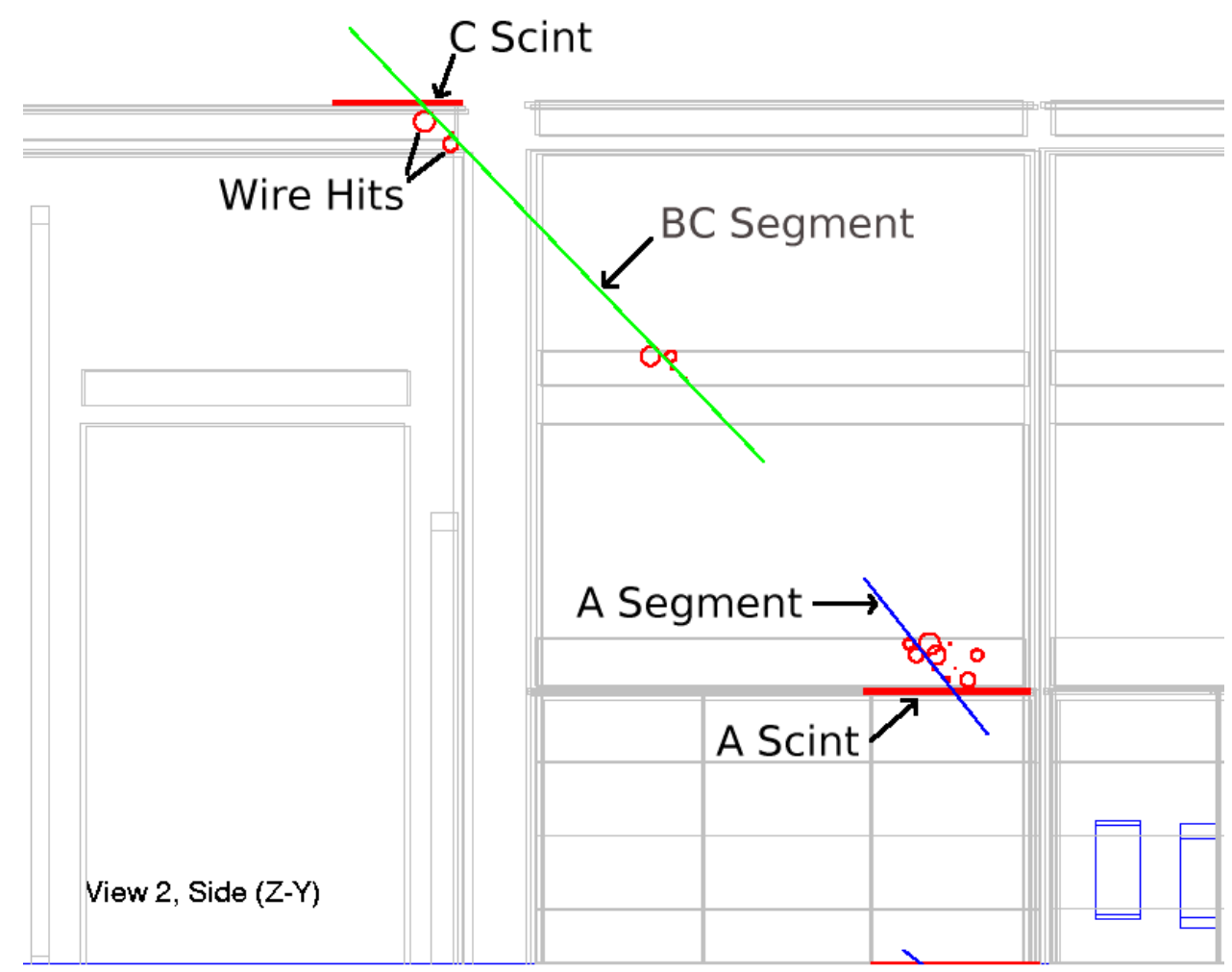

Figure 6.1. An event display of a Monte Carlo muon showing the wire hits, segments, and scintillator hits.

Figure 6.1 shows an event display of a muon reconstructed in the muon system. The individual wire hits are displayed as circles, indicating the drift time of the hit around each wire. The A- and BC- segments are shown inside and outside of the toroid. Finally, the confirming scintillator hits are shown in the A- and C-layers as horizontal lines (there are no B-layer scintillators in this region). Once all of the segments have been found, matches between A- and BC-layer segments are attempted. The bend angle of the muon between the A segment and the BC segment gives a local measurement of the muon $\mathrm{P}_{\mathrm{T}}$. 


\subsubsection{Central Track Match}

The matching of a local muon to a central track is performed in two ways. If the fit for the local muon track converged $\left(\chi^{2}>0\right)$, the muon segment is propagated to the central tracker. If no fit is found for the local muon $\left(\chi^{2}=-1\right)$, then the central track is propagated to the muon system. In both cases, a match in $\Delta \eta$ and $\Delta \phi$ is performed and the closest central track is assigned to the muon.

\subsubsection{Muon Quality Cuts}

The full set of cuts applied to muons in this analysis is

- $\mathrm{nseg}= \pm 3(\mathrm{~A}+\mathrm{BC}$ segment $)$ medium muons from MuoCandidate

- Match to track in central tracker

- Good central track $\left(\chi^{2} / N D F<4\right)$

- Loose cosmic cut based on scintillator times $\left(\left|t_{A}, t_{B C}\right|<10 \mathrm{~ns}\right)$

A medium muon is required to have the following minimum number of hits in the muon system:

- $\geq 1$ A-layer scintillator hit

$\bullet 2$ A-layer wire hits

- $\geq 1$ BC-layer scintillator hit

- $\geq 2$ BC-layer wire hits

In addition, two cuts are applied to the muon vertex (Section 6.7.2) to further eliminate muons from cosmic rays and to ensure that the muon comes from the primary event vertex. 
Tight muons, which were used for track-finding studies in data (Section 7.2.2), are the same as medium muons except the BC-layer wire hit requirement is $\geq 3$ and the local fit to the muon between the A- and BC-layers must converge.

\subsection{Offline Jet Identification}

The b-quarks from the decay of the $t \bar{t}$ pairs will fragment and produce a shower of hadrons and electromagnetic particles in the calorimeter. This shower of particles is called a jet, and the jet energy and direction are assigned to the original parton. Jets are reconstructed by summing the energy deposited in the calorimeter within a fixed cone. Corrections are made to the jet energy to account for the non-linear calorimeter response to low energy particles, dead regions, shower development outside of the jet cone, and pile-up from previous events. Several quality cuts are also applied to avoid reconstructing jets from individual noisy calorimeter cells.

\subsubsection{Jet Finding}

This analysis uses the improved legacy cone algorithm to find jets in the calorimeter [46]. In the cone algorithm, the energy of the jet is defined as the sum of energy deposited in the calorimeter cells within a fixed radius $\mathrm{R}$, where

$$
R=\sqrt{\left(\Delta \eta^{2}+\Delta \phi^{2}\right)}=0.5
$$

The cone algorithm begins by finding $0.1 \times 0.1 \eta-\phi$ towers that have $\mathrm{E}_{\mathrm{T}}>1.0$ $\mathrm{GeV}$. These towers are combined into preclusters in a cone of size $\mathrm{R}=0.3$ around the highest energy tower. The direction of the precluster is the $\mathrm{E}_{\mathrm{T}}$ weighted center of all towers in the precluster.

Jets are found around the preclusters by summing all towers within a cone of size $\mathrm{R}=0.5$ of the original precluster direction. A new $\mathrm{E}_{\mathrm{T}}$ weighted jet direction is then found for the 0.5 cone jet. If the direction is the same as the original precluster 
direction, the jet direction is stable. Otherwise, a new jet center is assigned, and the process repeats until the direction of the jet is stable. The minimum $\mathrm{E}_{\mathrm{T}}$ of a jet is 8 $\mathrm{GeV}$.

If towers are shared by two jets, the jets can either be merged or split. It the shared $\mathrm{E}_{\mathrm{T}}$ is $>50 \%$ of the lowest jet $\mathrm{E}_{\mathrm{T}}$, the jets are merged and a new jet direction is found. Otherwise, the jets are split, with the shared towers assigned to the closest jet.

\subsubsection{Jet Corrections}

After jet finding, corrections must be applied to the jet energy because of non-linear calorimeter response to low energy particles, dead regions, shower development outside of the jet cone, and pile-up from previous events. These are referred to as the jet energy scale (JES) corrections and were applies using the JetCorr v5.1 package in top_analyze [47]. The corrected energy of the jet is given by

$$
E_{\text {Corr }}=\frac{E_{\text {Meas }}-O}{R \times S}
$$

$O$ is an energy offset that corrects for energy in the jet that did not come from the initial parton. This includes activity from the uranium absorbing plates, energy from previous events (pile-up), and electronics noise. $O$ is measured using minimum-bias events and is parameterized in calorimeter detector $\eta$. $R$ is the calorimeter response to jets, and is measured in $\gamma+$ jet events by requiring that the jet energy balance the photon energy. $\mathrm{S}$ is the fraction of the jet energy that is deposited inside the jet cone and is determined in data by looking at jet profiles.

The errors on the JES correction include statistical and systematic errors from data and Monte Carlo as given by the JetCorr package. The four errors are combined in quadrature to give a total JES uncertainty. To study the effect of this uncertainty on signal and background efficiencies, the jet $\mathrm{E}_{\mathrm{T}}$ in Monte Carlo is increased or 
decreased by the fractional uncertainty on the correction. The fractional increase or decrease in the number of Monte Carlo events passing the jet cuts is taken as the JES error on the efficiency.

\subsubsection{Jet Quality Cuts}

A large number of fake jets are found by the jet finding algorithm. These fake jets can arise from hot cells in the calorimeter (a single cell with large fake energy), hot towers, and noise from various sources. To eliminate these fake jets, a standard set of cuts are applied:

1. $0.05<\mathbf{E M F}<\mathbf{0 . 9 5}$ The Electromagnetic Fraction (EMF) is the fraction of the jet $\mathrm{E}_{\mathrm{T}}$ that is contained in the EM portion of the calorimeter. This cut is designed to eliminate jets arising from a hot cell in either the hadronic or electromagnetic portion of the calorimeter that dominates the total jet energy.

2. $\mathbf{C H F}<\mathbf{0 . 4}$ The Coarse Hadronic Fraction $(\mathrm{CHF})$ is the fraction of the jet energy contained in the coarse hadronic region of the calorimeter. Jets typically deposit most of their energy in the inner fine hadronic portion of the calorimeter. Jets with a large fraction of their energy in the coarse hadronic region are typically due to noise in that region and are rejected with this cut.

3. HotF $<\mathbf{1 0}$ The Hotcell Fraction $(\mathrm{HotF})$ is defined as the ratio of $\mathrm{E}_{\mathrm{T}}$ for the most energetic cell in the jet and the next most energetic cell. By requiring that the most energetic cell have $<10 x$ the energy of the second most energetic, jets that come from a single hot cell are eliminated.

4. $\mathbf{n} 90>\mathbf{1}$ The number of towers containing $90 \%$ of the jet energy is required to be $>1$, eliminating jets that come from a single hot tower in the calorimeter.

To further reduce the effects of isolated cells, the 'T42' algorithm was used, which eliminate isolated low energy cells [48]. Specifically, cells with an energy less than four 
sigma above the pedestal value are removed if the cell does not have a neighbor with energy above the four sigma cut. Also, all negative energy cells are removed from the event. This cut reduces the total number of 'hit' cells in the event by $30-60 \%$.

Finally, noise due to readout electronics was reduced by requiring that jets found in the event be confirmed by a Level 1 jet tower. Since the data acquisition path for the trigger information and the precision readout for the calorimeter information is separate after the pre-amplifiers, this will eliminate jets arising from noise in the precision readout chain. This cut is over $99 \%$ efficient and reduces the number of noise jets by a factor of four after the T42 cut is applied.

\subsection{Missing Transverse Energy}

Neutrinos in an event deposit no energy in the detector. Their presence can be inferred, however, by missing energy in the transverse plane, which is denoted by $E_{T}$. The $E_{T}$ is a vector equal in magnitude and opposite in direction to the vector sum of all calorimeter cells that have passed the T42 cut.

Muons deposit only minimum ionizing energy in the calorimeter (typically about $2 \mathrm{GeV}$ ) and corrections must be made to the $\mathbb{E}_{T}$ in events with muons. The muon energy deposition in the calorimeter is removed from the $\not_{T}$ sum, and then the muon momentum is subtracted from the $\not_{T}$.

\subsection{Preselection Cuts}

After applying the above cuts, which are designed to select good muons and jets, a set of preselection cuts are applied to muons and jets to select events with topologies of $t \bar{t}$ pairs decaying into dimuons.

1. Two or more high $\mathbf{P}_{\mathrm{T}}$ isolated muons: The muons are required to have $\mathrm{P}_{\mathrm{T}}>15 \mathrm{GeV} / \mathrm{c}$ and be isolated (Section 6.7.1) from jets in the event. The two highest $\mathrm{P}_{\mathrm{T}}$ muons are also required to have opposite charge. 
2. 2 or more high $\mathbf{E}_{\mathrm{T}}$ jets: Events must have at least two jets with $\mathrm{E}_{\mathrm{T}}>20$ $\mathrm{GeV}$.

3. Prompt muon requirements: To ensure that the muons in the event originated from the primary vertex, two cuts are used to force the muon to be close to the primary vertex along the $\mathrm{Z}$ axis and in the $r-\phi$ plane (Section 6.7.2). The primary vertex must also have at least three tracks associated with it and occur within the active area of the SMT $(|Z|<60 \mathrm{~cm})$.

\subsubsection{Muon Isolation}

The muons from the decay of the $\mathrm{W}$ bosons tend to be isolated from jets in the event. To distinguish muons from the decay of $\mathrm{W}$ bosons from those arising from the decay of heavy quarks, both muons should be isolated from jet activity. A simple

requirement would be a $\Delta R=\sqrt{\Delta \eta^{2}+\Delta \phi^{2}}$ cut between the muon and all jets in the event. Other possibilities include a cut on the sum of the calorimeter energy in a cone around the muon (so-called 'Halo'), or the sum of the track $\mathrm{P}_{\mathrm{T}}$ in a cone around the muon (so-called 'TrkHalo').

The different requirements were studied using $\mathrm{Z} / \gamma^{*} \rightarrow \mu \mu$ 'signal' events and muons in QCD 'background' events [49]. A single muon efficiency, defined as the ratio of the number of muons passing the isolation requirement to all muons, was formed for the different isolation requirements. The Halo and TrkHalo cuts were found to reject more background that the $\Delta R$ cut for a set signal efficiency of $90 \%$. The initial isolation requirements chosen were

$$
\operatorname{Halo}(0.1,0.4)<2.5 \mathrm{GeV}
$$

and

$$
\text { TrkHalo(0.5) }<2.5 \mathrm{GeV}
$$


where $\operatorname{Halo}(0.1,0.4)$ is the sum of the transverse energy in a hollow cone about the muon [50]. The 0.1 and 0.4 indicate the inner and outer radius of the cone in units of $\Delta R=\sqrt{\Delta \eta^{2}+\Delta \phi^{2}}$ from the muon axis. The inner limit on the cone is used to exclude the minimum ionizing energy from the muon in the sum. TrkHalo(0.5) is the sum of the $\mathrm{P}_{\mathrm{T}}$ of the tracks around the muon out to a radius of $\mathrm{R}=0.5$.

Further studies showed that a more efficient cut involved the ratio of the Halo and TrkHalo to the muon $\mathrm{P}_{\mathrm{T}}$. Two new isolation variables, rat11 and rattrk, were defined as

$$
\begin{aligned}
\text { rat11 } & =\operatorname{Halo}(0.1,0.4) / P_{T} \mu \\
\text { rattrk } & =\operatorname{TrkHalo}(0.5) / P_{T} \mu
\end{aligned}
$$

The isolation requirement used is rat11 $<0.12$ and rattrk $<0.12$. The value of the cut was chosen to give the same background rejection as the original cuts and gave an increase in the efficiency for both muons to pass the isolation requirement of about $11 \%[51]$.

\subsubsection{Prompt Muon Cuts}

To ensure that the muons in the event came from the primary vertex, two cuts were used. Along the beam axis, the $\mathrm{Z}$ position of the muon vertex is required to be within $1 \mathrm{~cm}$ of the primary vertex. In the $r-\phi$ plane, a cut on the muon Distance of Closest Approach (DCA) significance is used. The DCA is the closest distance between the

muon track and the Z-axis in the $r-\phi$ plane, and the significance is defined as the ratio of the DCA and its error:

$$
D C A_{\text {Sig }}=\left|D C A_{\text {muon }}\right| / \sigma_{D C A}<3
$$




\subsection{Selection Cuts}

After preselection cuts, four more cuts are applied to the data to increase the signal to background ratio.

1. $\Delta \phi\left(\mu_{\text {leading }}, E_{T}\right)$ : The opening angle between the muon with the highest $\mathrm{P}_{\mathrm{T}}$ (leading muon) and the $\mathbb{E}_{T}$ is required to be $<165^{\circ}$. This cut removes events where a poorly reconstructed muon $\mathrm{P}_{\mathrm{T}}$ gives a large value for the muon-corrected $E_{T}$ in the opposite direction. Figure 6.2 shows the invariant mass as a function of the $E_{T}$ for $\mathrm{Z} / \gamma^{*}$ Monte Carlo events before and after the $\Delta \phi$ cut. Note the reduction in high $E_{T}$ /high mass events in the upper right corner of the right plot.
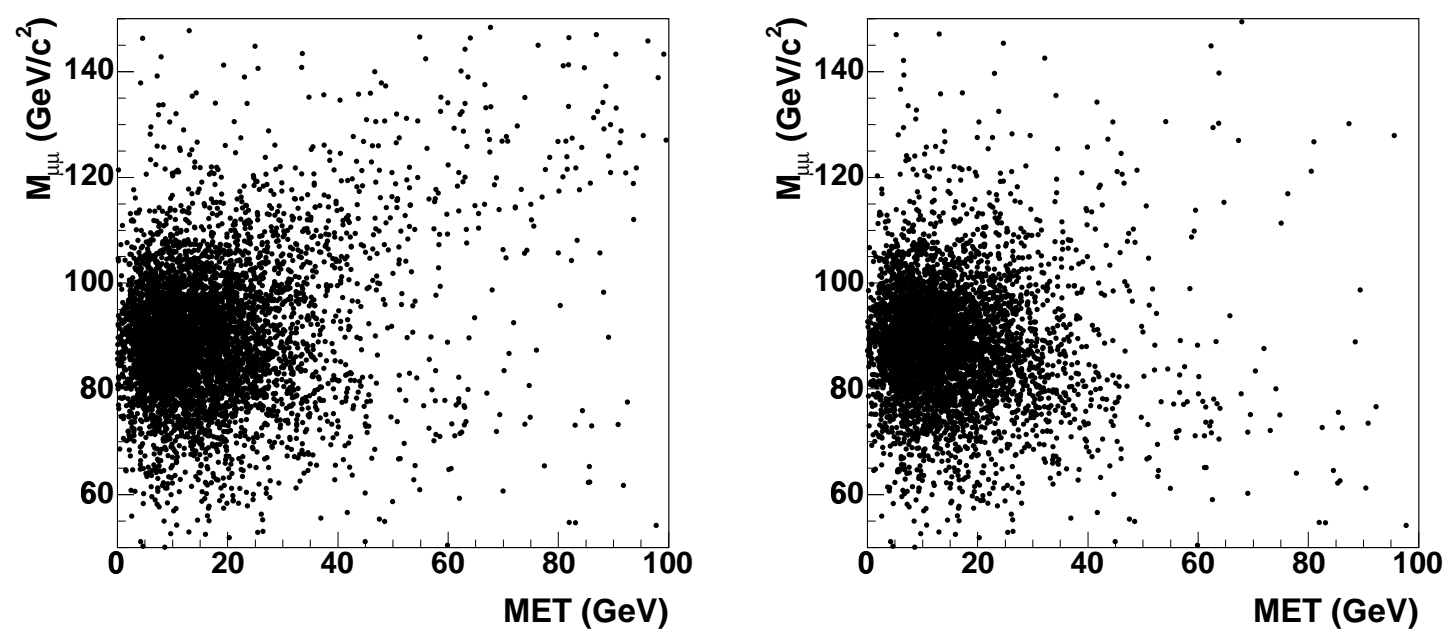

Figure 6.2. Dimuon invariant mass versus $E_{T}$ for $\mathrm{Z} / \gamma^{*} \mathrm{MC}$ without the $\Delta \phi$ cut (left) and with. Events with poorly reconstructed muons tend to have large invariant mass and $E_{T}$.

2. $\mathbf{H}_{T} \mu$ : The large mass of the top quark leads to large amounts of total energy for a given event. $\mathrm{H}_{T} \mu$ is defined as the scaler sum of the jet $\mathrm{E}_{\mathrm{T}}$ for all jets with $\mathrm{E}_{\mathrm{T}}>15 \mathrm{GeV}$ and $|\eta|<2.5$ and the $\mathrm{P}_{\mathrm{T}}$ of the leading muon. The requirement 
of $\mathrm{H}_{T} \mu>120 \mathrm{GeV}$ comes from a grid search optimization described in Section 8.5 .

3. Invariant mass: The reconstructed muon resolution causes $\mathrm{Z} / \gamma^{*} \rightarrow \mu \mu$ events to have some $E_{T}$ and the tails of this distribution are very large. For this reason, events with a dimuon invariant mass in the $\mathrm{Z}$ boson mass range $\left(70 \mathrm{GeV} / \mathrm{c}^{2}<\right.$ $\mathrm{M}_{\mu \mu}<110 \mathrm{GeV} / \mathrm{c}^{2}$ ) are excluded. The limits represent the roughly 2 sigma values of the fit $\mathrm{Z}$ boson mass in data events (Figure 6.3).

4. Missing Transverse Energy: The neutrinos from the decay of the two W bosons cause a large amount of missing transverse energy $\left(E_{T}\right)$ in the event. This analysis requires $\mathbb{E}_{T}>35 \mathrm{GeV}$, which is very effective in removing $\mathrm{Z} / \gamma^{*} \rightarrow \mu \mu$ events that have no real $E_{T}$. The choice of this cut value is described in Section 8.5 .

$E_{T}$

\subsection{Monte Carlo Samples}

Monte Carlo events were used to determine the efficiency for signal $t \bar{t}$ events to pass the selection criteria (Chapter 7) and to estimate the number of background events in the data set (Chapter 8). The signal Monte Carlo was ALPGEN $t \bar{t}$ events where both $\mathrm{W}$ bosons decayed to leptons. The background samples include $\mathrm{Z} / \gamma^{*}$ plus two jet events where the $\mathrm{Z} / \gamma^{*}$ decayed to two leptons and WW plus two jet events where both W bosons decayed to leptons. In addition, a signal Monte Carlo sample identical to the default sample except with the 'TuneA' underlying event turned on was generated to study the effects of the underlying event. The details of the Monte Carlo parameters for the signal and background samples are given in Table 6.3.

Two other Monte Carlo samples were used for determining data/Monte Carlo corrections. A $t \bar{t}$ sample where one $\mathrm{W}$ boson decayed to a lepton and the other to 


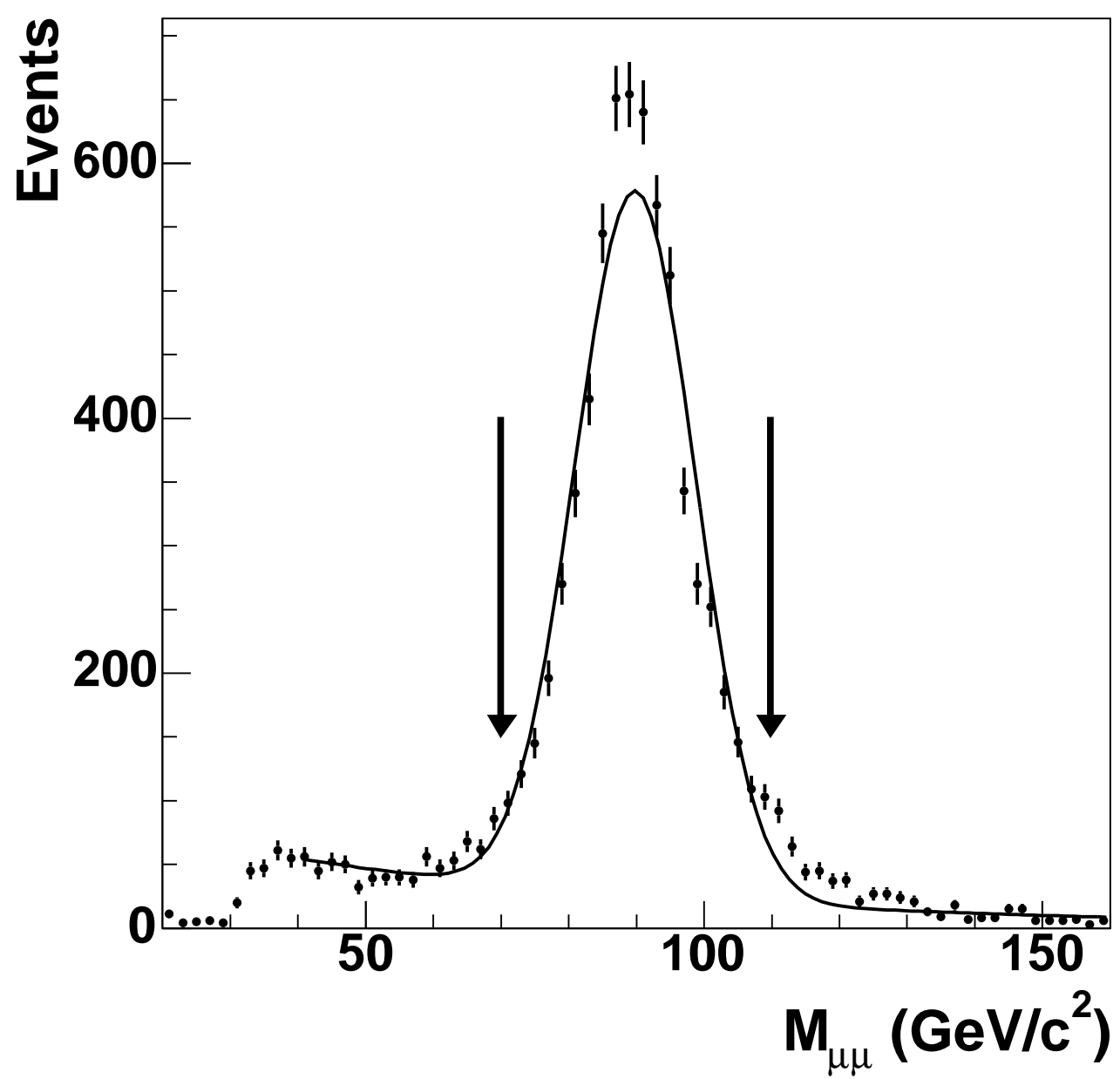

Figure 6.3. The invariant mass of dimuon events in data. The fit shown is a combined Gaussian and exponential. The mean and width of the Gaussian are 89.1 $\mathrm{GeV} / \mathrm{c}^{2}$ and $8.9 \mathrm{GeV} / \mathrm{c}^{2}$, respectively. The arrows indicate the chosen cuts. 


\begin{tabular}{|l|l|l|l|}
\hline Parameters & Signal $t \bar{t}$ & $Z / \gamma^{*}+$ 2 jets & $W W+2$ jets \\
\hline Generator & ALPGEN & ALPGEN & ALPGEN \\
Decay & WW $\rightarrow 1 \mathrm{l}$, & $\mathrm{Z} / \gamma^{*} \rightarrow \mathrm{ll}$, & $\mathrm{WW} \rightarrow \mathrm{ll}$, \\
& $(\mathrm{l}=\mathrm{e}, \mu, \tau)$ & $(\mathrm{l}=\mathrm{e}, \mu, \tau)$ & $(\mathrm{l}=\mathrm{e}, \mu, \tau)$ \\
& $\tau \rightarrow$ anything & $\tau \rightarrow \mathrm{e}, \mu$ & $\tau \rightarrow$ anything \\
$\mathrm{Q}^{2}$ & $\mathrm{M}_{t}^{2}$ & $\mathrm{M}_{l l}^{2}$ & $\mathrm{M}_{W}^{2}$ \\
$\mathrm{PDF}$ & $\mathrm{CTEQ} 6.1 \mathrm{M}$ & CTEQ $6.1 \mathrm{M}$ & $\mathrm{CTEQ6.1 \textrm {M }}$ \\
Min. Bias & 0.8 & 0.8 & 0.8 \\
Underlying Event & None & None & None \\
$\mathrm{P}_{\mathrm{T}}$ (parton) & No Cut & $>12 \mathrm{GeV}$ & $>12 \mathrm{GeV}$ \\
$|\eta|$ (parton) & No Cut & $<2.7$ & $<2.7$ \\
$\Delta \mathrm{R}$ (parton,parton) & No Cut & $>0.4$ & $>0.4$ \\
$\mathrm{P}_{\mathrm{T}}$ (lepton) & No Cut & $>12 \mathrm{GeV}$ & $>12 \mathrm{GeV}$ \\
$|\eta|$ (lepton) & No Cut & $<2.7$ & $<2.7$ \\
\hline
\end{tabular}

TABLE 6.3. Details of the Monte Carlo samples used in this analysis. The $\mathrm{Z} / \gamma^{*}$ Monte Carlo was divided into three samples based on the invariant mass of the lepton pair. The mass ranges were 15 to $60 \mathrm{GeV} / \mathrm{c}^{2}, 60-130 \mathrm{GeV} / \mathrm{c}^{2}$, and $>130 \mathrm{GeV} / \mathrm{c}^{2}$. A signal $t \bar{t}$ sample with identical parameters but with the 'TuneA' multiple parton interactions included was also generated.

\begin{tabular}{|l|l|l|}
\hline Parameters & Lepton + jets $\bar{t} \bar{t}$ & $Z / \gamma^{*} \rightarrow \mu \mu$ \\
\hline Generator & ALPGEN & PYTHIA \\
Decay & WW $\rightarrow 1 q \bar{q},(1=\mathrm{e}, \mu, \tau)$ & $\mathrm{Z} / \gamma^{*} \rightarrow \mu \mu$ \\
& $\tau \rightarrow$ anything & \\
$\mathrm{Q}^{2}$ & $\mathrm{M}_{t}^{2}$ & $\mathrm{M}_{l l}^{2}$ \\
$\mathrm{PDF}$ & CTEQ 6.1M & CTEQ4L \\
Underlying Event & None & TuneA \\
$\mathrm{P}_{\mathrm{T}}$ (parton) & None & None \\
$|\eta|$ (parton) & None & None \\
$\Delta \mathrm{R}$ (parton,parton) $)$ & None & None \\
$\mathrm{P}_{\mathrm{T}}$ (lepton) & None & None \\
$|\eta|$ (lepton) & None & None \\
\hline
\end{tabular}

TABLE 6.4. Details of Monte Carlo samples used for muon reconstruction efficiency studies (Lepton + jets $t \bar{t}$ ) and track finding efficiency studies (PYTHIA Z $/ \gamma^{*} \rightarrow \mu \mu$ ). 
a $q \bar{q}$ pair (lepton + jets channel) was used for muon reconstruction efficiency studies (Section 7.2.1). A PYTHIA Z $/ \gamma^{*} \rightarrow \mu \mu$ sample was used for track finding efficiency studies (Section 7.2.2). The details of these samples are given in Table 6.4.

The $\mathrm{Z} / \gamma^{*}$ Monte Carlo was simulated in three mass ranges, $15<\mathrm{M}_{l l}<60 \mathrm{GeV} / \mathrm{c}^{2}$ (mass1), $60<\mathrm{M}_{l l}<130 \mathrm{GeV} / \mathrm{c}^{2}$ (mass2), and $\mathrm{M}_{l l}>130 \mathrm{GeV} / \mathrm{c}^{2}$ (mass3). The $\mathrm{Z} / \gamma^{*}$ was forced to decay to two leptons $\left(\mathrm{e}^{+} \mathrm{e}^{-}, \mu^{+} \mu^{-}\right.$, or $\left.\tau^{+} \tau^{-}\right)$, and the $\tau$ 's were forced to decay to either e or $\mu$. The branching ratio for $\tau \rightarrow e$ is $17.8 \%$, while for $\tau \rightarrow \mu$ it is $17.4 \%$. The fractional number of events for each decay channel in the final sample can be expressed as

$$
1=f_{Z \rightarrow e e}+f_{Z \rightarrow \mu \mu}+0.03\left(f_{Z \rightarrow \tau \tau \rightarrow e e}\right)+0.03\left(f_{Z \rightarrow \tau \tau \rightarrow \mu \mu}\right)+0.06\left(f_{Z \rightarrow \tau \tau \rightarrow e \mu}\right)
$$

where $f$ is the fraction of each decay channel in the final sample, the factor of 0.03 is the branching ratio for $\tau \rightarrow l$ squared, and the factor of 0.06 for the e $\mu$ channel is from the charge-conjugated decay. The total contribution of each channel is given in Table 6.5. The smaller contributions from $Z \rightarrow \tau^{+} \tau^{-}$events arise because only those events where both $\tau$ 's decay to leptons are kept.

\begin{tabular}{|l|l|}
\hline Decay & Fraction in Final Sample \\
\hline$Z \rightarrow e^{+} e^{-}$ & $47.1 \%$ \\
\hline$Z \rightarrow \mu^{+} \mu^{-}$ & $47.1 \%$ \\
\hline$Z \rightarrow \tau^{+} \tau^{-} \rightarrow e^{+} e^{-}$ & $1.45 \%$ \\
\hline$Z \rightarrow \tau^{+} \tau^{-} \rightarrow \mu^{+} \mu^{-}$ & $1.45 \%$ \\
\hline$Z \rightarrow \tau^{+} \tau^{-} \rightarrow e \mu$ & $2.9 \%$ \\
\hline
\end{tabular}

TABle 6.5. Z boson decays in $\mathrm{Z} / \gamma^{*}$ Monte Carlo.

The total cross section times branching fraction $(\sigma \times \mathrm{BR})$ for the sample is the sum of $\sigma \mathrm{x}$ BR for the five decay channels. Table 6.6 gives $\sigma \times \mathrm{BR}$ for the three mass windows for $\mathrm{Z} / \gamma^{*} \rightarrow$ ee (labeled ALPGEN) and for the entire sample (labeled combined). If there was no requirement on the tau decay, the conversion factor $\kappa$ 


\begin{tabular}{|l|c|c|c|c|}
\hline $\begin{array}{l}\text { Monte Carlo } \\
\text { Sample }\end{array}$ & Mass $\left(G e V / c^{2}\right)$ & $\begin{array}{c}\text { ALPGEN } \\
\sigma x B R(p b) \\
\left(\mathrm{Z} / \gamma^{*} \rightarrow \mathrm{ee}\right)\end{array}$ & $\begin{array}{c}\text { Combined } \\
\sigma x B R(p b)\end{array}$ & $\begin{array}{c}\text { Events } \\
\text { Generated }\end{array}$ \\
\hline$t \bar{t} \rightarrow \mathrm{ll}$ & & & 0.75 & 43000 \\
\hline $\mathrm{Z} / \gamma^{*}$ mass1 & $15-60$ & 2.95 & 6.27 & 122400 \\
\hline $\mathrm{Z} / \gamma^{*}$ mass2 & $60-130$ & 12.6 & 26.7 & 144600 \\
\hline $\mathrm{Z} / \gamma^{*}$ mass3 & $>130$ & 0.136 & 0.29 & 95900 \\
\hline $\mathrm{WW}$ & & & 0.29 & 19500 \\
\hline
\end{tabular}

TABLE 6.6. Monte Carlo samples used for this analysis with the Monte Carlo cross section times branching fraction and the number of generated events. For the $\mathrm{Z} / \gamma^{*}$ events, the value for the ALPGEN $\sigma \times \mathrm{BR}$ is the cross section times branching fraction for $\mathrm{Z} / \gamma^{*} \rightarrow$ ee. The combined cross section is the ALPGEN cross section times the conversion factor $\kappa$ of 2.123 , which accounts for the five decay channels in the sample (Equation 6.9).

between the ALPGEN cross section (for one lepton flavor) and the entire sample would be three. Because the tau is forced to decay to an electron or muon, the conversion factor $\kappa$ is given by

$$
\begin{aligned}
\kappa & =1(e e)+1(\mu \mu)+0.03(\tau \tau \rightarrow e e)+0.03(\tau \tau \rightarrow \mu \mu)+0.06(\tau \tau \rightarrow e \mu) \\
& =2.12
\end{aligned}
$$




\section{Chapter 7}

\section{EFFICIENCIES}

The cross section, $\sigma_{t \bar{t}}$, is found by

$$
\sigma_{t \bar{t}}=\frac{\left(N_{o b s}-N_{b k g}\right)}{\epsilon_{s i g} \times B R\left(t \bar{t} \rightarrow \mu^{+} \mu^{-}\right) \times \mathcal{L}_{I n t}}
$$

where $\mathrm{N}_{o b s}$ and $\mathrm{N}_{b k g}$ are the total observed events in data and the expected number of background events respectively, $\epsilon_{\text {Sig }}$ is the total efficiency for signal events to pass all preselection and selection cuts, $B R(t \bar{t} \rightarrow \mu \mu)$ is the branching ratio for both $\mathrm{W}$ bosons from the decay of $t \bar{t}$ pairs to decay to muons (including the cascade $W \rightarrow \tau \rightarrow \mu$ ), and $\mathcal{L}_{\text {Int }}$ is the total integrated luminosity.

The total efficiency, $\epsilon_{\text {sig }}$, is divided into five categories: muon identification (ID), jet ID, prompt muon, trigger, and selection efficiencies. The cuts associated with these five categories are described in Chapter 6. The muon ID, jet ID, and prompt muon cuts are collectively called preselection cuts and select muons and jets that are consistent with those expected from signal events. The trigger efficiency is the fraction of signal events that are collected by the online trigger requirement. Finally, the selection cuts use specific event topologies to increase the signal-to-background ratio.

The efficiencies for these cuts were studied using signal Monte Carlo and are summarized in Table 7.1. In addition to the various cut efficiencies, four scale factors, denoted by $\kappa$, were measured to to correct for differences between the data and Monte Carlo. The efficiencies and scale factors for the muon ID, jet ID, prompt muon, trigger, and selection cuts are described in Sections 7.2 to 7.6 .

All cut efficiencies were taken from the ALPGEN signal Monte Carlo events (Section 6.9) and are cumulative, taken in the order shown in Table 7.1. Each cut efficiency 


\begin{tabular}{c|c|c|c}
\hline Category & Cut & Efficiency & Cumulative efficiency \\
\hline Muon ID & $\geq 2$ muons (opp. charge) & $0.448 \pm 0.006$ & $0.448 \pm 0.0061$ \\
& Isolation & $0.665 \pm 0.009$ & $0.298 \pm 0.0056$ \\
& $\geq 2$ muons, $\mathrm{P}_{\mathrm{T}}>15 \mathrm{GeV}$ & $0.817 \pm 0.009$ & $0.243 \pm 0.0053$ \\
& $\kappa_{\text {MuonReco }}$ & 1.020 & $0.248 \pm 0.0054$ \\
& $\kappa_{\text {Track-matching }}$ & $0.964 \pm 0.006$ & $0.239 \pm 0.0054$ \\
& $\kappa_{\text {Isolation }}$ & $0.858 \pm 0.049$ & $0.205 \pm 0.0126$ \\
\hline Trigger & & $0.920 \pm 0.007$ & $0.189 \pm 0.0116$ \\
\hline Jet ID & $\geq 2$ jets & $0.854 \pm 0.009$ & $0.161 \pm 0.0101$ \\
& $\geq 2$ jets, $\mathrm{E}_{\mathrm{T}}>20 \mathrm{GeV}$ & $0.945 \pm 0.006$ & $0.153 \pm 0.0096$ \\
\hline Prompt & $|z|<60, N_{\text {trk }}>2$ & $0.983 \pm 0.004$ & $0.149 \pm 0.0094$ \\
Muons & $d c a$ Cut & $0.864 \pm 0.010$ & $0.128 \pm 0.0082$ \\
& $\Delta z(\mu, P V)$ & $0.997 \pm 0.002$ & $0.128 \pm 0.0082$ \\
& $\kappa_{d c a}$ & 0.982 & $0.126 \pm 0.0081$ \\
\hline Selection & $\Delta \phi\left(\mu_{\text {leading }}, F_{T}\right)$ & $0.845 \pm 0.011$ & $0.106 \pm 0.0070$ \\
& $H_{T} \mu$ & $0.968 \pm 0.006$ & $0.103 \pm 0.0068$ \\
& $M_{\mu \mu}$ & $0.673 \pm 0.016$ & $0.069 \pm 0.0049$ \\
& $E_{T}$ & $0.825 \pm 0.016$ & $0.057 \pm 0.0042$ \\
\hline & Total & $\mathbf{0 . 0 5 7} \pm \mathbf{0 . 0 0 4 2}$ \\
\hline
\end{tabular}

TABLE 7.1. Breakdown of efficiencies for $t \bar{t} \rightarrow \mu \mu$ events (where the muon is allowed to come from $W$ or $\tau$ ) and the corresponding scale factors to account for the difference in efficiency measured in data and Monte Carlo. Errors are statistical only.

is defined as

$$
\epsilon_{C u t}=\frac{N_{\text {Cut }}}{N_{\text {Sample }}}
$$

where $N_{\text {Sample }}$ is the number of Monte Carlo events after all previous cuts and $N_{C u t}$ is the number of events in $N_{\text {Sample }}$ that pass the cut in question. A total of 6646 Monte Carlo $t \bar{t} \rightarrow \mu \mu$ events were generated, including muons from the decay $W \rightarrow \tau \rightarrow \mu$. After all cuts, 452 events remain for a total efficiency (before scale factors) of $6.8 \%$. After all scale factors have been included, the final efficiency is $5.7 \%$. 


\begin{tabular}{|c|c|}
\hline Number & Description \\
\hline 0 & Number of primary vertex tracks $\geq 3$ \\
1 & $|Z|$ primary vertex $<60 \mathrm{~cm}$ \\
2 & Number of medium muons $\geq 2$ \\
3 & Two highest $\mathrm{P}_{\mathrm{T}}$ muons isolated \\
5 & Two highest $\mathrm{P}_{\mathrm{T}}$ muons have $\mathrm{P}_{\mathrm{T}}>15 \mathrm{GeV} / \mathrm{c}$ \\
6 & DCA significance for two lead muons $<3$ \\
7 & Two lead muons have opposite sign \\
8 & $|\Delta|$ between muons and primary vertex $<1 \mathrm{~cm}$ \\
9 & Number of jets $\geq 2$ \\
10 & Two lead jets have $\mathrm{E}_{\mathrm{T}}>20 \mathrm{GeV}$ \\
11 & Invariant mass of two lead muons $<70 \mathrm{GeV} / \mathrm{c}^{2}$ or $>110 \mathrm{GeV} / \mathrm{c}^{2}$ \\
12 & $\Delta \phi\left(\mu_{\text {leading }}, E_{T}\right)<165$ degrees \\
13 & $\mathrm{H}_{T} \mu>120 \mathrm{GeV}$ \\
14 & Monte Carlo trigger efficiency \\
15 & $\not_{T}>35 \mathrm{GeV}$ \\
16 & Monte Carlo tracking efficiency \\
17 & Not used \\
18 & Tag dimuon signal Monte Carlo, both W's decayed to muons \\
19 & At least one of two lead muons is isolated \\
20 & Tag dimuon signal Monte Carlo, two muons from either W or tau \\
\hline
\end{tabular}

TABLE 7.2. List of cut numbers in the analysis program diMu.

\subsection{Cut Order in Analysis Program diMu}

The analysis program that analyzes the Root trees is called diMu and is used to apply the cuts above and implement any data/Monte Carlo corrections that are necessary. Table 7.2 summarizes the twenty cuts in diMu. Table 7.3 lists the cuts that were used for the terms in Table 7.1. Only one of the scale factors, $\kappa_{\text {Track-matching }}$, is derived from diMu. The other three were derived in other studies performed on data and Monte Carlo. 


\begin{tabular}{|c|c|c|}
\hline Category & Cut & diMu Cut Numbers \\
\hline Muon ID & $\geq 2$ muons (opp. charge) & 2,6 \\
& Isolation & 3 \\
& $\geq 2$ muons, $\mathrm{P}_{\mathrm{T}}>15 \mathrm{GeV}$ & 4 \\
& $\kappa_{\text {MuonReco }}$ & - \\
& $\kappa_{\text {Track-matching }}$ & 15 \\
& $\kappa_{\text {Isolation }}$ & - \\
\hline Trigger & $\geq 2$ jets & 13 \\
\hline Jet ID & 2 jets, $\mathrm{E}_{\mathrm{T}}>20 \mathrm{GeV}$ & 8 \\
& $|z|<0, N_{\text {trk }}>2$ & 9 \\
\hline Prompt Muons & $d c a$ Cut & 0,1 \\
& $\Delta z(\mu, P V)$ & 5 \\
& $\kappa_{\text {dca }}$ & 7 \\
\hline Selection & $\Delta \phi\left(\mu_{\text {leading }}, \not_{T}\right)$ & 11 \\
& $H_{T} \mu$ & 12 \\
& $M_{\mu \mu}$ & 10 \\
& $E_{T}$ & 14 \\
\hline
\end{tabular}

TABLE 7.3. Summary of analysis program diMu cut numbers used for the various efficiencies listed in Table 7.1. 


\subsection{Muon ID}

Two high $\mathrm{P}_{\mathrm{T}}$, isolated muons are expected in the dimuon decay channel of $t \bar{t}$ pairs. The efficiency for an event to have two muons that pass all muon preselection cuts (Section 6.7) can be expressed as

$$
\epsilon_{\text {MuonID }}=\epsilon_{\text {MuonReco }} \times \epsilon_{\text {MuonIso }} \times \epsilon_{\text {Muon } P_{T}}
$$

where $\epsilon_{\text {MuonReco }}$ is the efficiency to reconstruct two medium track-matched muons with opposite sign, $\epsilon_{\text {MuonIso }}$ is the efficiency for the two leading muons in the event to pass the isolation requirements, and $\epsilon_{M u o n P_{T}}$ is the efficiency for having two muons with $\mathrm{P}_{\mathrm{T}}>15 \mathrm{GeV} / \mathrm{c}$.

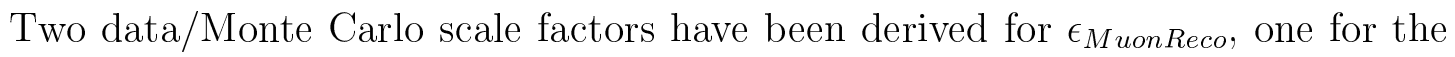
efficiency for reconstructing local muons (Section 7.2.1) and one for the central track finding efficiency (Section 7.2.2). In addition, a scale factor for $\epsilon_{\text {MuonIso }}$ has been derived (Section 7.2.3). The muon ID, isolation, and $\mathrm{P}_{\mathrm{T}}$ efficiencies, and the three scale factors, are summarized in Table 7.1 .

\subsubsection{Muon Reconstruction Data/Monte Carlo Scale Factor}

A correction is made between data and Monte Carlo for the muon reconstruction efficiency. To determine the reconstruction efficiency in data, a sample of $\mathrm{Z} / \gamma^{*} \rightarrow$ $\mu \mu$ data events was collected with a single muon trigger. One muon that passed the quality cuts outlined in Section 6.4.3 was found and matched to a single Level 2 muon trigger object in the event with a $\Delta \mathrm{R}$ cut. Next, a second muon was reconstructed using only the central tracker by requiring that the charge and invariant mass were consistent with a $\mathrm{Z}$ boson $\left(80 \mathrm{GeV} / \mathrm{c}^{2}<\mathrm{M}_{\mu \mu}<100 \mathrm{GeV} / \mathrm{c}^{2}\right)$. The reconstruction efficiency was then the fraction of these 'track-only' muons that were also reconstructed by the muon system. The efficiencies are calculated as a function of $\mathrm{P}_{\mathrm{T}}, \eta$, and $\phi$. 
This study was also done in Monte Carlo using PYTHIA Z $/ \gamma^{*} \rightarrow \mu \mu$ events (Section 6.9). The data/Monte Carlo scale factor was parameterized in $\mathrm{P}_{\mathrm{T}}$, muon detector $\eta$, and $\phi$ by taking the ratio of the data and Monte Carlo efficiencies [43]. In general, there is good agreement between the data and the Monte Carlo reconstruction efficiencies, with the scale factor close to one and flat in $\mathrm{P}_{\mathrm{T}}$ and $\phi$.

A fit of the correction factor in muon detector $\eta$ was used with $t \bar{t}$ Monte Carlo in the lepton + jets channel (Section 6.9) to determine an overall scale factor, $\kappa_{\text {MuonReco }}$, of 1.01 per muon. The systematic error due to the scale factor $\kappa_{\text {MuonReco }}$ was obtained by varying the fit for the scale factor in muon detector $\eta$ within its $\pm 1 \sigma$ errors. The resulting change in the overall scale factor as described above was determined to be $1.6 \%$ per muon, giving a total error of $3.2 \%$ for the dimuon channel.

\subsubsection{Track Finding Data/Monte Carlo Factor}

A similar study was performed to determine the difference between data and Monte Carlo in the central track reconstruction efficiency [52]. A sample of tight local muons (Section 6.4.3) from the dimuon data skim was used. In addition, the muons were required to have a matching MIP trace in the calorimeter, tight scintillator timing cuts, and isolation from jets in the event. The data were separated into two groups, before and after a hardware timing change in the CFT in September of 2003. For the Monte Carlo, a sample of PYTHIA Z $/ \gamma^{*} \rightarrow \mu \mu$ events was used (Section 6.9).

For each isolated muon, a search for a matching central track was performed in the 'muon window'. The muon window was a $\pm 0.3 \times \pm 0.2 \eta-\phi$ window centered around the muon position. The raw track-finding efficiency, $\epsilon_{m}$, was defined as the ratio of the number of muons with at least one matching central track to the total number of muons. The probability for finding a random track match, $\epsilon_{r}$, defined as a matching central track caused by a charged particle other than the muon, was determined by searching for a matching track to the muon in an $\pm 0.3 \times \pm 0.2 \eta-\phi$ 
window adjacent in $\phi$ to the muon window. The 'true' efficiency, $\epsilon_{t}$, is given by

$$
\epsilon_{t}=\frac{\epsilon_{m}-\epsilon_{r}}{1-\epsilon_{r}}
$$

After correcting for the random track matches, a fit to the efficiency was performed in $\eta$ and $\phi$ for the data and the Monte Carlo. The ratio of these plots was then used as the correction factor, $\kappa_{\text {Tracking }}$. The results were implemented in a macro detailed in [52]. For each muon reconstructed in Monte Carlo, a probability was returned by the macro that the muon would have a matching track reconstructed in data and compared to a random number. If the probability was larger than the random number, the event was said to have a matching track. The per event probability of having two or more track matched muons is

- $\kappa_{\text {Tracking }}=.950$ (Old timing)

- $\kappa_{\text {Tracking }}=.972($ New timing $)$

- $\kappa_{\text {Tracking }}=.964$ (Luminosity Weighted Average)

The sources of systematic error on $\kappa_{\text {Tracking }}$ include cosmic rays, trigger bias, $\mathrm{P}_{\mathrm{T}}$ variations, and muons from QCD. The macro also accounts for the effect of the statistical error from each $\eta-\phi$ bin and returns the total error for a given sample. The luminosity-weighted average error for the entire sample is $2.1 \%$.

\subsubsection{Muon Isolation Data/Monte Carlo Scale Factor}

To measure the difference between data and Monte Carlo for the isolation requirement, a data sample of $\mathrm{Z} / \gamma^{*} \rightarrow \mu \mu$ events was used, along with ALPGEN Z/ $\gamma^{*}$ Monte Carlo (Section 6.9). The isolation efficiency is defined as the fraction of muons in the $\mathrm{Z} / \gamma^{*}$ events that pass the isolation requirements outlined in Section 6.7.1. The per muon efficiency in data was $84.1 \%$, while the Monte Carlo efficiency was $90.2 \%$. The ratio between data and Monte Carlo efficiencies gives a scale factor, $\kappa_{\text {MuonIso }}$, of 0.926 . 
The systematic error associated with this scale factor comes from the difference in the $\Delta \mathrm{R}(\mu$,jet $)$ distribution for $\mathrm{Z} / \gamma^{*}$ events and $t \bar{t}$ signal events. To account for this, the data/Monte Carlo scale factor from the $\mathrm{Z} / \gamma^{*}$ events was parameterized in $\Delta \mathrm{R}(\mu$,jet). This curve was used to re-weight the signal Monte Carlo and determine the new efficiency. There was a net change of $3 \%$ in the efficiency per muon, giving a total error of $6 \%$.

\subsection{Trigger Efficiency}

The Level 1 and Level 2 trigger efficiencies were measured using $\mathrm{Z} / \gamma^{*} \rightarrow \mu \mu$ events in the $\mathrm{Z}$ boson mass range. The $\mathrm{Z} / \gamma^{*}$ events were used to ensure a clean sample of dimuon events that were close to the environment expected for $t \bar{t}$ events (two high $\mathrm{P}_{\mathrm{T}}$, isolated muons.) The sample required two muons with opposite charge and an invariant mass between 70 and $110 \mathrm{GeV} / \mathrm{c}^{2}$. The Level 1 and Level 2 trigger efficiencies were combined to get the overall trigger efficiency using signal Monte Carlo events.

The Level 1 requirement is a dimuon tight scintillator trigger (mu2ptxatxx). To remove bias due to events that occurred on a dimuon trigger, the $\mathrm{Z} / \gamma^{*} \rightarrow \mu \mu$ events were collected with a single muon trigger. One muon was randomly selected as a bias muon and matched in region and octant to a single muon hardware trigger at Level 1. The second muon was used as the test, or unbiased, muon and a match in region and octant to a second Level 1 muon hardware trigger was attempted. The single muon Level 1 trigger efficiency is

$$
\epsilon_{L 1}=\frac{N_{\text {Trig }}}{N_{\text {Muons }}}
$$

where $N_{\text {Muons }}$ is the total number of unbiased muons and $N_{\text {Trig }}$ is the number of unbiased muons with a matching Level 1 trigger. The efficiency was parameterized as a function of muon detector $\eta$ and $\phi$. 
The dimuon trigger efficiency was formed as the product of the single muon trigger efficiencies for the muons in an event. This technique assumes that all events with two single muon triggers in the Level 1 readout will fire the dimuon trigger. This was confirmed in data by verifying that the raw dimuon trigger input (AndOr term) at the trigger framework fired in all events with two muons matched to single muon triggers, i.e., that the algorithm for forming dimuon triggers from single muon triggers in Level 1 is $100 \%$ efficient. If the two muons are close $(\Delta R<0.5)$, an inefficiency would arise from the dimuon counting scheme in the L1MU trigger (Section 4.4.1). However, the muon isolation requirement (Section 6.7.1) imposes a cut of $\Delta \mathrm{R}>0.5$ between the two muons due to the TrkHalo requirement, so this inefficiency does not arise.

The single muon Level 2 efficiency was measured in the same manner as Level 1. In this case, the biased muon was also required to have a matching Level 2 muon. If the unbiased muon had a matching Level 1 trigger, then a match was attempted between the unbiased muon and the Level 2 trigger result. The single muon efficiency is defined in the same manner as Level 1. Figure 7.1 shows the trigger efficiency parameterizations at Level 1 and Level 2 for muons with $\mathrm{P}_{\mathrm{T}}$ above $15 \mathrm{GeV} / \mathrm{c}$.

Over half of the data collected also had a Level 3 trigger requirement (Section 6.2). In both trigger list $\mathrm{v} 11$ and $\mathrm{v} 12$, a high $\mathrm{P}_{\mathrm{T}}$ muon or high $\mathrm{P}_{\mathrm{T}}$ track was required at Level 3. These efficiencies were measured in dimuon data events that passed the Level 1 and Level 2 trigger requirements and had two high $\mathrm{P}_{\mathrm{T}}$ muons. The efficiency of the two Level 3 triggers OR'd together was found to be $99.5 \%$ in trigger list v11, and $99.3 \%$ in trigger list v12. The luminosity-weighted average for the entire sample (including the triggers with no Level 3 requirement) is $99.7 \%$, and was taken to be $100 \%$ for this analysis.

The parameterizations for the Level 1 and Level 2 efficiencies shown in Figure 7.1 were incorporated in the standard TriggerEfficiency class as part of the top_trigger package [53]. To determine the total efficiency, the TriggerEfficiency class would loop 

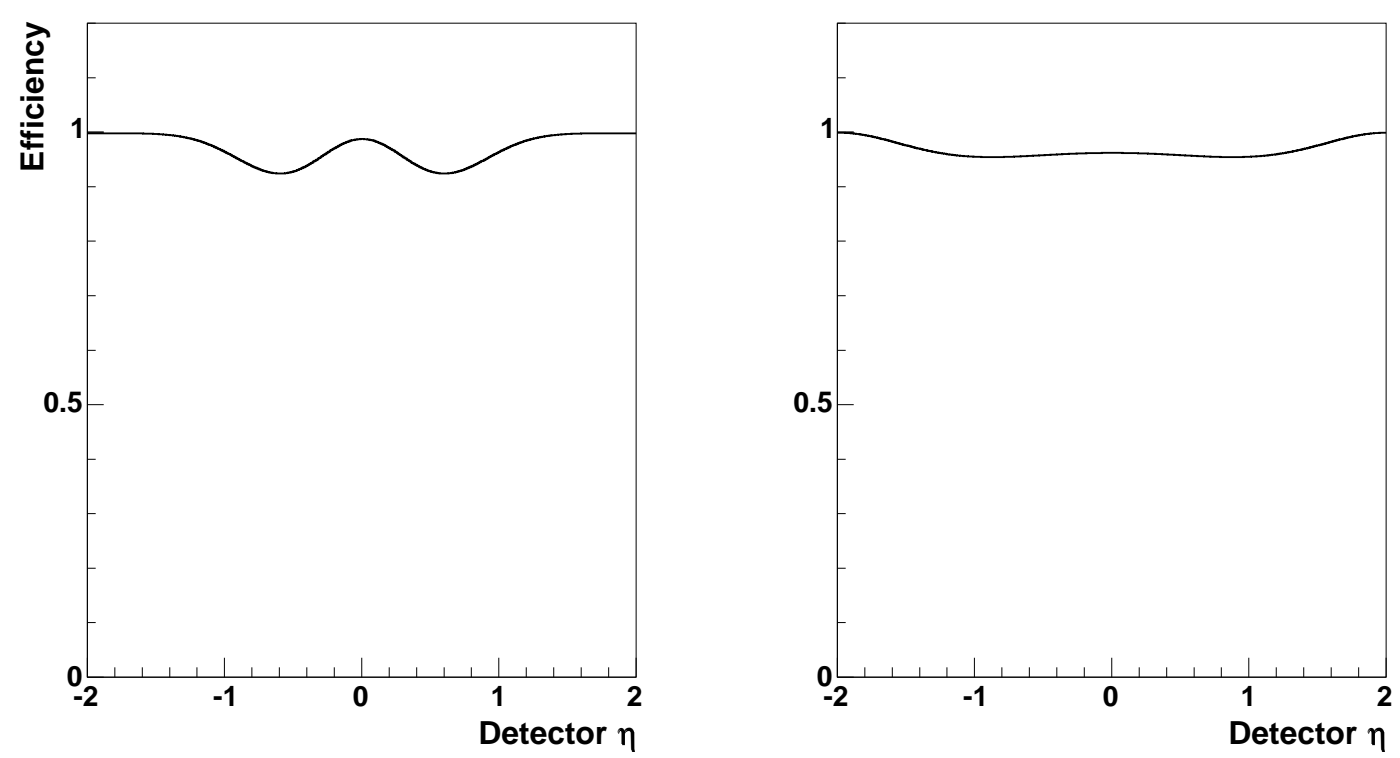

FIgURE 7.1. The Level 1 and Level 2 single muon trigger efficiency parameterizations used in determining the overall trigger efficiency for Monte Carlo events.

over all reconstructed muons in the event and return an event weight equal to the probability that at least two muons in the event would fire the Level 1 trigger and at least one muon would fire the Level 2 trigger. If the event weight was greater than a random number, the event was said to have passed the trigger. In signal Monte Carlo events with at least two isolated muons with $\mathrm{P}_{\mathrm{T}}>15 \mathrm{GeV} / \mathrm{c}$, the total efficiency was found to be $92.0 \%$.

The trigger systematic error was derived by allowing the trigger parameterization curves for the Level 1 and Level 2 trigger efficiencies to vary by $\pm 1 \sigma$ and determining the effect on the signal Monte Carlo efficiency. Variations in the Level 1 efficiency had a $1 \%$ effect, while variations in Level 2 had no effect, giving a total error of $1 \%$ on the trigger efficiency. 


\subsection{Jet ID}

Two energetic jets from the decay of the b-quarks are also expected in signal events. Signal Monte Carlo was used to determine the efficiency for events passing the jet preselection cuts (Section 6.7). This efficiency is

$$
\epsilon_{J e t I D}=\epsilon_{J e t R e c o} \times \epsilon_{J e t E_{T}}
$$

where $\epsilon_{\text {JetReco }}$ is the efficiency to reconstruct two or more jets that pass the basic jet ID cuts outlined in Section 6.5 with $|\eta|<2.5$ and $\mathrm{E}_{\mathrm{T}}>15 \mathrm{GeV}$, and $\epsilon_{J e t E_{T}}$ is the efficiency for two or more jets to have $\mathrm{E}_{\mathrm{T}}>20 \mathrm{GeV}$. The efficiencies are listed in Table 7.1 .

There are two systematic errors associated with jet ID. The first is a difference in the jet finding efficiency between data and Monte Carlo. These differences are mainly due to the increase in jet trigger coverage from $|\eta|<2.4$ to $|\eta|<3.2$ that occurred during data taking that is not simulated in the Monte Carlo. To account for these differences, the jet reconstruction efficiencies were measured in $\gamma+$ jet events in data and Monte Carlo. The difference between data and Monte Carlo was used to make an alternate signal Monte Carlo file (so called 'recoeff') that contained the same Monte Carlo events with re-weighting applied to the jet reconstruction. There was a $6.2 \%$ drop in total signal efficiency with this alternate Monte Carlo sample, which was taken as the systematic error.

The second systematic error comes from the overall Jet Energy Scale (JES, Section 6.5.2) and was determined by allowing the JES corrections to the $\mathrm{E}_{\mathrm{T}}$ of signal Monte Carlo jets to vary within their $1 \sigma$ errors. The change in the overall efficiency was taken as the systematic error and was measured to be $+6 \% /-10 \%$. 


\subsection{Prompt Muons}

The primary vertex is required to have at least three tracks associated with it and to occur at a value of $|Z|<60 \mathrm{~cm}$, which is the active area for the SMT. The efficiency for this cut is determined from signal Monte Carlo and is defined to be the ratio of the number of Monte Carlo events that have a primary vertex satisfying the above criteria to all Monte Carlo events. The efficiency was found to be $97.7 \%$. A data/Monte Carlo scale factor of 0.997 was derived by taking the ratio of the vertex efficiency in $\mathrm{Z} / \gamma^{*}$ data and ALPGEN Monte Carlo events (Section 6.9). The product of the signal Monte Carlo efficiency and the data/Monte Carlo scale factor is $97.4 \%$. The statistical uncertainty on the scale factor of $0.8 \%$ is taken as the systematic error on this efficiency. More details of vertex studies can be found in [54].

The muons from the decay of the $\mathrm{W}$ bosons are expected to come from the primary vertex. To further eliminate muons from cosmic rays and muons from the decay of heavy quarks, the distance in $\mathrm{Z}$ between the muon and the primary vertex is required to be $<1 \mathrm{~cm}$. The efficiency for this cut was measured with signal Monte Carlo to be $99.7 \%$.

The Distance of Closest Approach (DCA) significance (Section 6.7.2) cut requires that the muons lies close to the Z-axis in the $r-\phi$ plane. This efficiency was measured in signal Monte Carlo events to be $86.4 \%$. A per muon data/Monte Carlo scale factor, $\kappa_{D C A}$, was derived for the DCA cut by comparing the DCA significance cut efficiency in $\mathrm{Z} / \gamma^{*}$ data and Monte Carlo. The per muon ratio, $\epsilon_{D C A}($ Data $) / \epsilon_{D C A}($ MonteCarlo $)$ is 0.991 , giving a per event scale factor $\kappa_{D C A}$ of 0.982 . The statistical error on this correction factor of 0.022 per muon is taken as a systematic error for the total efficiency. 

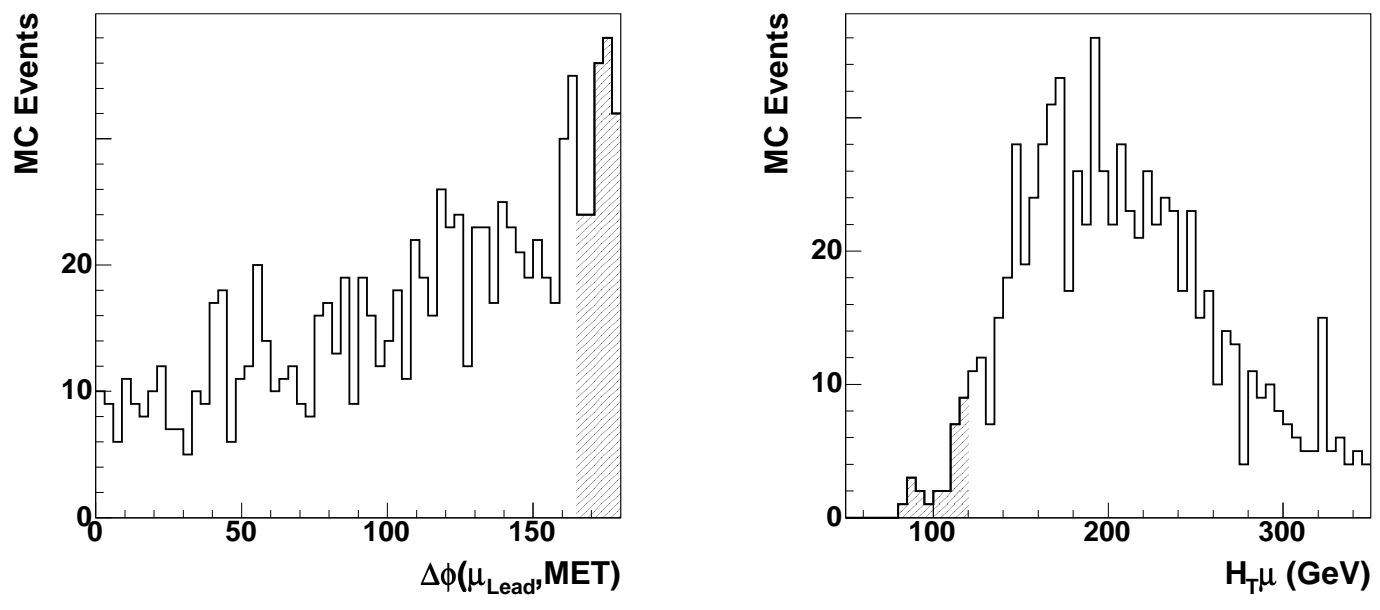

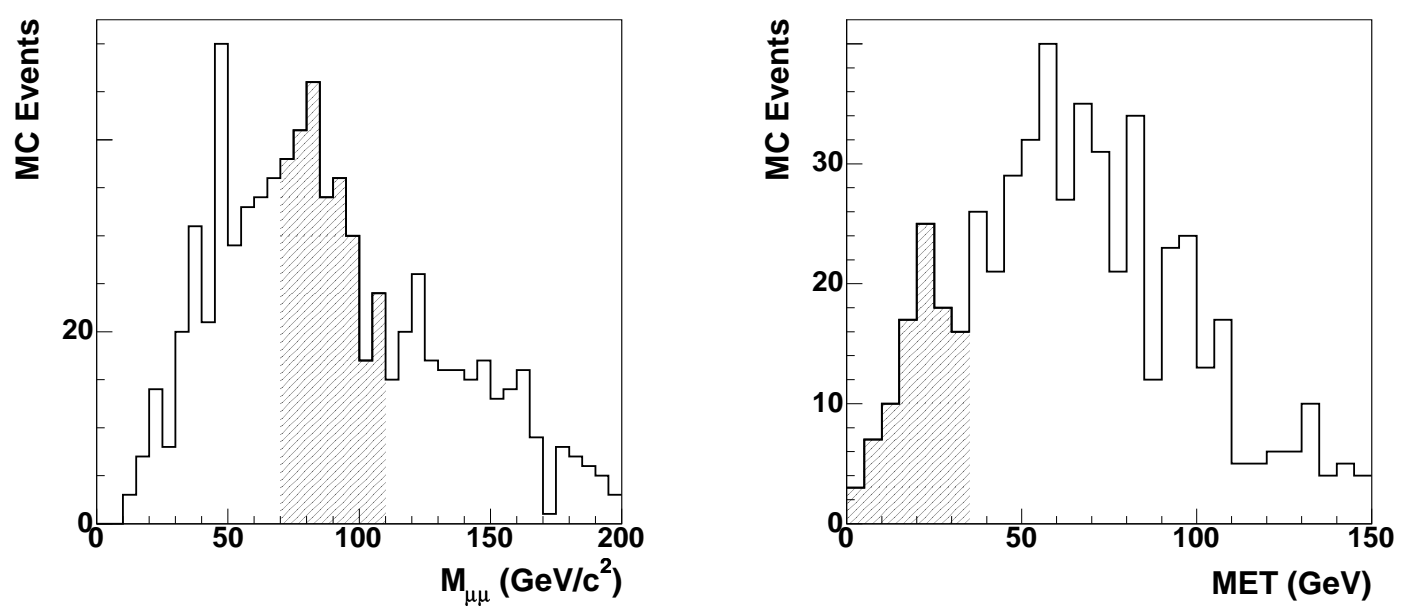


\begin{tabular}{|c|c|c|c|}
\hline Parameter & Fraction & Source & Section \\
\hline$\mu$ ID & $3 \%$ & $\mathrm{Z} / \gamma^{*}$ events $\mathrm{w} /$ unbiased muon & 7.2 .1 \\
\hline$\mu$ isolation & $6 \%$ & $\begin{array}{l}\text { Reweighting study } \\
\text { w/signal Monte Carlo }\end{array}$ & 7.2 .3 \\
\hline$\mu$ tracking & $2 \%$ & Data/Monte Carlo tracking macro & 7.2 .2 \\
\hline L1 $\mu$ trigger & $1 \%$ & Trigger macro & 7.3 \\
\hline Jet ID & $-6 \%$ & Reweighted Monte Carlo to reflect data & 7.4 \\
\hline$\mu \sigma_{d c a}$ & $4 \%$ & Statistical error on $\kappa_{D C A}$ & 7.5 \\
\hline JES & $(+6-10) \%$ & JES macro & 7.4 \\
\hline tuneA & $1 \%$ & Alternate Monte Carlo & 7.7 \\
\hline top mass & $6 \%$ & Alternate Monte Carlo & 7.7 \\
\hline Total & $+12-16 \%$ & & \\
\hline No JES & $+10-12 \%$ & & \\
\hline
\end{tabular}

TABLE 7.4. Summary of the relative systematic uncertainties on the $t \bar{t} \rightarrow \mu \mu$ efficiencies.

generated (so-called 'tuneA', Section 6.9). The change in efficiency was less than $1 \%$.

Table 7.4 summarizes the systematic errors measured for this analysis. The largest errors are due to uncertainties in the jet energy scale, the top quark mass, the jet reconstruction efficiency, and the muon isolation efficiency. 


\section{ChAPTER 8 BACKGROUND Estimation}

In addition to the $t \bar{t}$ signal events, several background processes can lead to events that pass all of the selection criteria and contribute to the final data sample. These include

events with Z bosons or Drell-Yan virtual photons that decay to two muons (labeled $\mathrm{Z} / \gamma^{*}$ events) with associated jets. Events with two $\mathrm{W}$ bosons, where both $\mathrm{W}$ bosons decay to muons, and associated jets (labeled WW events) can also contribute to the final data set. Finally, events with one muon from a $\mathrm{W}$ boson and a second muon from the decay of a heavy quark or events with two muons from heavy quark decay (collectively called WQCD events) can also contribute to the background sample. This chapter describes the backgrounds considered in this analysis and the gives the expected amount of each background in the final data set.

\subsection{Background Processes and Monte Carlo Samples}

The backgrounds considered for this analysis are:

- $\mathrm{Z} / \gamma^{*} \rightarrow \mu^{+} \mu^{-}+$multijet: These events produce two isolated, high $\mathrm{P}_{\mathrm{T}}$ muons and associated jets, but there is no real $\mathbb{E}_{T}$ from a neutrino in the event. The measured $E_{T}$ can be significant, however, due to unclustered calorimeter energy or fluctuations in the measured jet energies. Poorly reconstructed muons also contribute to the measured $E_{T}$.

- $\mathrm{Z} / \gamma^{*} \rightarrow \tau^{+} \tau^{-}+$multijet, $\tau \rightarrow \mu \nu \bar{\nu}$ : These events have two isolated high $\mathrm{P}_{\mathrm{T}}$ muons and real $E_{T}$, but the branching fraction for both taus to decay to muons is small $(3 \%)$. The muons typically have lower transverse momentum than muons in signal events and $\mathrm{Z} / \gamma^{*} \rightarrow \mu^{+} \mu^{-}$. 
- $\mathrm{WW} \rightarrow \mu^{+} \mu^{-}+$multijet: These events have a similar final-state signature to $t \bar{t}$ events, with two muons and two neutrinos, but the cross section times branching fraction is very small (less than the expected signal). The associated jets tend to have less energy than signal events as well, since the jets in signal events are from energetic b-quarks from the decay of the top quark.

- $\mathrm{W}+$ heavy quark multijet QCD (WQCD): These events have at most one real isolated muon from the decay of the $\mathrm{W}$ boson. Muons associated with the decay of heavy quark jets (charm and bottom), however, can fake the isolation requirement and give two isolated muons in the event.

The $\mathrm{Z} / \gamma^{*}$ and WW backgrounds were studied using Monte Carlo events described in Section 6.9. All selection cuts were applied to the Monte Carlo samples to get an overall efficiency for each sample to pass the cuts. This was combined with the Monte Carlo cross section and the integrated luminosity to estimate the number of events for each sample in the data. Additionally, the $\mathrm{Z} / \gamma^{*}$ and WW Monte Carlo were used with signal Monte Carlo events to optimize the $\mathbb{E}_{T}$ and $\mathrm{H}_{T} \mu$ selection cuts (Section 8.5) by maximizing the figure of merit (FOM), $\frac{s}{\sqrt{s+b}}$.

The WQCD background was estimated using data events and the matrix method (Section 8.4). This method combines the number of events in a tight sample (all selection cuts applied), the number in a loose sample (remove the isolation requirement for one muon), and the efficiency for signal and WQCD events to pass the muon isolation requirement (Section 6.7.1).

\section{$8.2 \mathrm{Z} / \gamma^{*}$}

The dominant background for this analysis is $\mathrm{Z} / \gamma^{*}$ events. Monte Carlo events are used to get an overall efficiency for this background to pass all selection criteria. This is combined with the Monte Carlo cross section and the data luminosity to 
estimate the number of expected background events. Finally, the number of expected background events from Monte Carlo are normalized to the data with a control sample of $\mathrm{Z} / \gamma^{*}$ events falling within the $\mathrm{Z}$ boson mass window of $70 \mathrm{GeV} / \mathrm{c}^{2}<\mathrm{M}_{\mu \mu}<110$ $\mathrm{GeV} / \mathrm{c}^{2}$.

\subsubsection{Raw Yields}

The expected (unnormalized) yields from Monte Carlo are given by:

$$
\text { Raw Yield }=\frac{M C_{\text {Pass }} \times \sigma_{M C} \times B R \times \mathcal{L}_{\text {Int }}}{M C_{\text {Total }}}
$$

where $M C_{\text {Pass }}$ is the number of Monte Carlo events passing all cuts, $\sigma_{M C} \times B R$ is the Monte Carlo cross section times branching ratio, $\mathcal{L}_{\text {Int }}$ is the integrated luminosity of the data sample, and $M C_{\text {Total }}$ is the total number of generated Monte Carlo events.

Table 8.1 shows the number of Monte Carlo events passing all cuts and the expected Monte Carlo yields for the $\mathrm{Z} / \gamma^{*}$ background samples before data normalization. The samples are divided into $\mathrm{Z} / \gamma^{*} \rightarrow \mu \mu$ and the cascade $\mathrm{Z} / \gamma^{*} \rightarrow \tau \tau \rightarrow \mu \mu$ by using the Monte Carlo information to tag the decay of the $\mathrm{Z} / \gamma^{*}$. Though the cascade decay has neutrinos from the decay of the taus, and thus has real $\mathbb{E}_{T}$, the low branching fraction for both tau particles to decay to muons (3\%) makes the $\mathrm{Z} / \gamma^{*} \rightarrow \mu \mu$ the dominant background.

\subsubsection{Z $/ \gamma^{*}$ Scale Factor}

To normalize the Monte Carlo prediction to the data, an independent control sample, labeled the $\mathrm{Z}$ control sample, of $\mathrm{Z} / \gamma^{*}$ events were collected in data. These events had all preselection cuts applied, along with the requirement that $\Delta \phi\left(\mu_{\text {leading }}, E_{T}\right)<$ 165 degrees. The mass of the dimuon pair was required to fall within the window 70 $\mathrm{GeV} / \mathrm{c}^{2}<M_{\mu \mu}<110 \mathrm{GeV} / \mathrm{c}^{2}$. To increase statistics, no $E_{T}$ or $\mathrm{H}_{T} \mu$ cut was applied. A total of 80 events satisfying these criteria were found in data. 


\begin{tabular}{|l|c|c|c|c|}
\hline Monte Carlo Sample & $\begin{array}{c}\text { Total MC } \\
\text { Events }\end{array}$ & $\begin{array}{c}\text { Events } \\
\text { Passing } \\
\text { All Cuts }\end{array}$ & $\sigma x$ BR $(p b)$ & Expected Yield \\
\hline $\mathrm{Z} / \gamma^{*} \rightarrow \mu \mu$, mass1 & 57632 & 57 & 2.95 & 0.408 \\
$\mathrm{Z} / \gamma^{*} \rightarrow \mu \mu$, mass2 & 68272 & 71 & 12.6 & 1.828 \\
$\mathrm{Z} / \gamma^{*} \rightarrow \mu \mu$, mass3 & 45388 & 523 & 0.136 & 0.219 \\
\hline $\mathrm{Z} / \gamma^{*} \rightarrow \tau \tau \rightarrow \mu \mu$, mass1 & 1786 & 0 & 0.00894 & 0.0 \\
$\mathrm{Z} / \gamma^{*} \rightarrow \tau \tau \rightarrow \mu \mu$, mass2 & 2057 & 6 & 0.0381 & 0.155 \\
$\mathrm{Z} / \gamma^{*} \rightarrow \tau \tau \rightarrow \mu \mu$, mass3 & 1360 & 3 & 0.000412 & 0.001 \\
\hline & & & Total & $\mathbf{2 . 6 1 1} \pm \mathbf{0 . 2 3}$ \\
\hline
\end{tabular}

TABLE 8.1. Estimated $\mathrm{Z} / \gamma^{*}$ yields from Monte Carlo before the data/Monte Carlo normalization. The $\mathrm{Z} / \gamma^{*}$ Monte Carlo samples have been tagged as the direct $\mathrm{Z} / \gamma^{*} \rightarrow$ $\mu \mu$ decay or the cascade decay $\mathrm{Z} / \gamma^{*} \rightarrow \tau \tau \rightarrow \mu \mu$ and represent a fraction of the total Monte Carlo sample described in Section 6.9. The decay $\mathrm{Z} / \gamma^{*} \rightarrow \mu \mu$ accounts for roughly $95 \%$ of the estimated background.

To normalize the $\mathrm{Z} / \gamma^{*}$ Monte Carlo to the data, a scale factor $\kappa_{Z}$ was introduced

$$
\kappa_{Z}=\frac{D a t a_{Z C}}{M C_{Z C}}
$$

where Data $_{Z C}$ is the number of $\mathrm{Z}$ control events in data and $M C_{Z C}$ is the expected number of events from Monte Carlo. Table 8.2 summarizes the number of data and Monte Carlo events in the Z control sample. The mass1 and mass3 Z $/ \gamma^{*}$ Monte Carlo events contribute less than 0.1 events in the $\mathrm{Z}$ control sample and were ignored. The value of the scale factor is

$$
\kappa_{Z}=0.781 \pm 0.088 \text { (stat.) }
$$

The scale factor is calculated without the $\mathrm{H}_{T} \mu$ or $\mathbb{E}_{T}$ cuts to increase statistics. To test the stability of $\kappa_{Z}$ with respect to cuts on $\mathrm{H}_{T} \mu$ and $\mathbb{E}_{T}$, the scale factor was plotted for the $\mathrm{Z}$ control sample with a range of cuts on both variables. Figure 8.1 shows the variation of $\kappa_{Z}$ for a value of the $\mathrm{H}_{T} \mu$ cut from 0 to $135 \mathrm{GeV}$ and for $E_{T}$ cuts from 0 to $45 \mathrm{GeV}$. The line indicates the value of $\kappa_{Z}$ used of 0.781 . The scale 


\begin{tabular}{|c|c|c|c|c|}
\hline $\begin{array}{c}\text { Monte Carlo } \\
\text { Events Passing }\end{array}$ & $\begin{array}{c}\text { Monte Carlo } \\
\sigma x B R(p b)\end{array}$ & $\begin{array}{c}\text { Monte Carlo } \\
\text { Yield }\end{array}$ & Data Events & $\begin{array}{c}\text { Scale Factor } \\
\kappa_{Z}\end{array}$ \\
\hline 3968 & 26.7 & 104.4 & 80 & $\mathbf{0 . 7 8 1} \pm \mathbf{0 . 0 8 8}$ \\
\hline
\end{tabular}

TABLE 8.2. The number of $\mathrm{Z} / \gamma^{*}$ mass2 Monte Carlo events, expected Monte Carlo yield, and the number of data events in the $\mathrm{Z}$ control sample. The mass1 and mass3 $\mathrm{Z} / \gamma^{*}$ Monte Carlo contribute less that 0.1 events and are ignored. The scale factor, $\kappa_{Z}$, is the ratio of the data yield to the Monte Carlo yield.

factor does not show any systematic increase or decrease as the cut values are varied.

Finally, comparisons were made between data and Monte Carlo for events in the $\mathrm{Z}$ control sample. In all cases, the Monte Carlo was normalized to the data by setting the area under the Monte Carlo distribution equal to 80, the number of data events. The results of the Kolmogorov-Smirnov test returned by Root are included on each plot. This number is the probability that the data are compatible with the Monte Carlo [55].

Figure 8.2 shows the leading and second jet $\mathrm{E}_{\mathrm{T}}$. The jets in the data, particularly the leading jet, appear to have less $\mathrm{E}_{\mathrm{T}}$ than the Monte Carlo. One possible cause of this is the parton-level cut of $12 \mathrm{GeV}$ applied to the jets in the Monte Carlo (Section 6.9). A reweighting study was done (Section 8.2.4) to determine the effect of this difference. Four other distributions are shown in Figure 8.3, including the leading muon $\mathrm{P}_{\mathrm{T}}, \mathrm{H}_{T} \mu$, the dimuon invariant mass $\left(\mathrm{M}_{\mu \mu}\right)$, and the $\mathrm{P}_{\mathrm{T}}$ of the dimuon pair (labeled $\mathrm{Z}_{\mathrm{T}}$ ). Good agreement is seen between data and Monte Carlo for these four variables.

\subsubsection{Z $/ \gamma^{*}$ Yields}

The final estimate for the total amount of $\mathrm{Z} / \gamma^{*}$ events in the data is the product of the estimated Monte Carlo yield (Equation 8.1) and the scale factor. The full Monte Carlo samples were used (Section 6.9) and no tagging was done to separate $\mathrm{Z} / \gamma^{*} \rightarrow \mu \mu$ and $\mathrm{Z} / \gamma^{*} \rightarrow \tau \tau \rightarrow \mu \mu$ decays. Table 8.3 summarizes the contributions of the three 

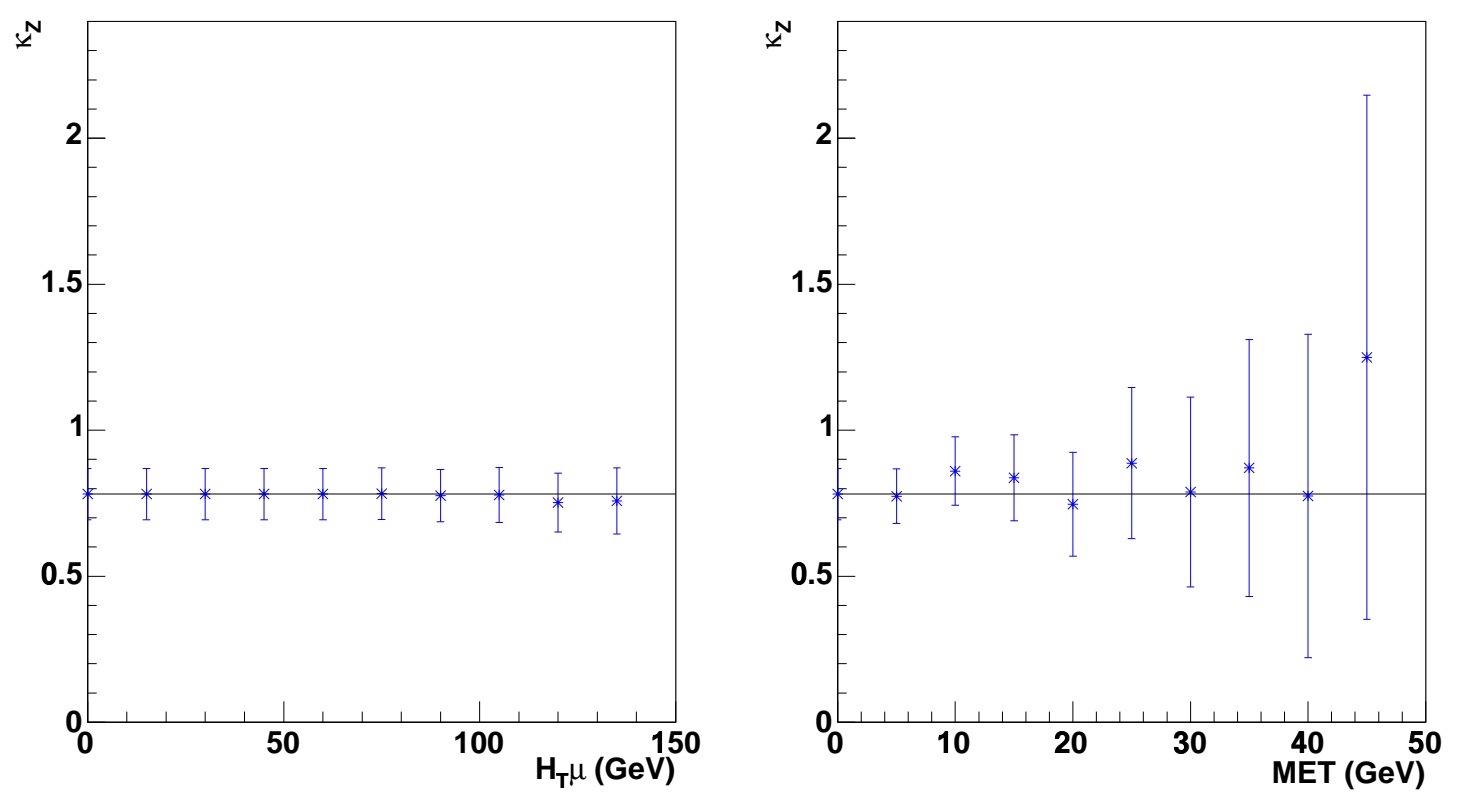

Figure 8.1. The data/Monte Carlo scale factor $\kappa_{Z}$ as a function of $\mathrm{H}_{T} \mu$, left, and $\not_{T}$, right. The line is drawn at the chosen value for $\kappa_{Z}$ of 0.781 .
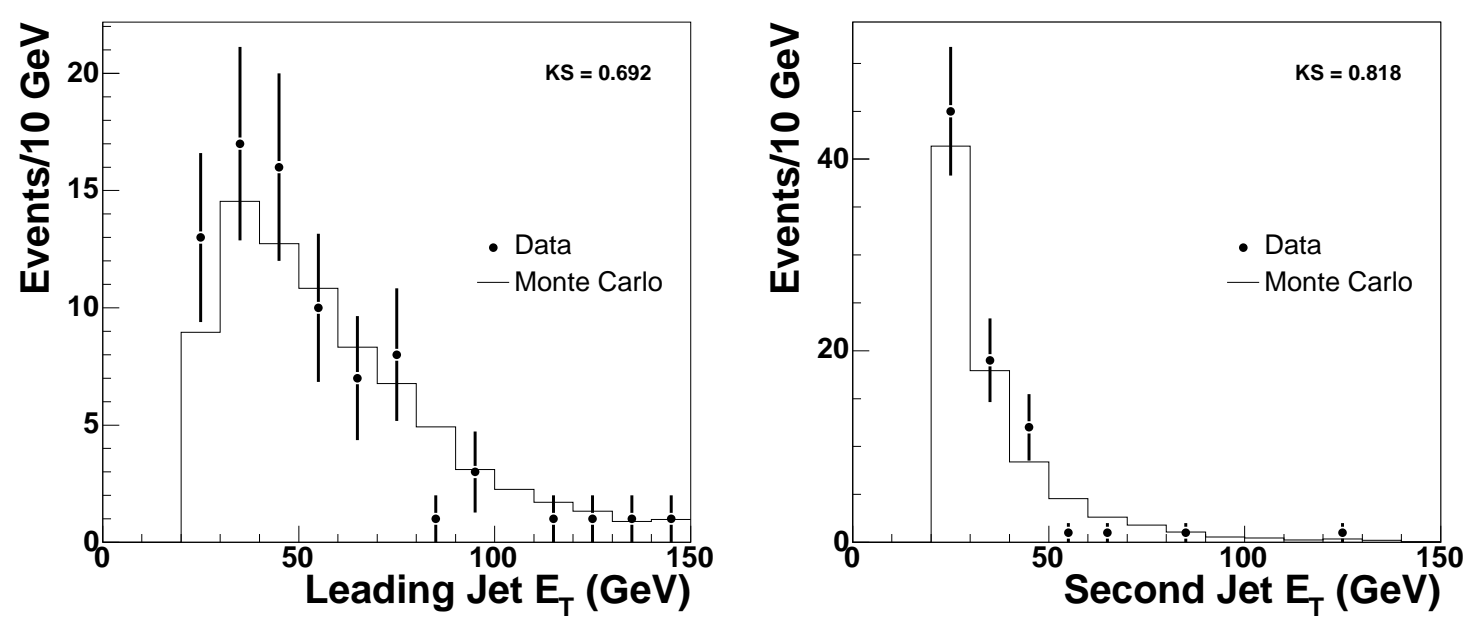

Figure 8.2. Comparison of the data (points) and Monte Carlo (histogram) distributions for the leading and second jet $\mathrm{E}_{\mathrm{T}}$. The data have more events at low $\mathrm{E}_{\mathrm{T}}$. 

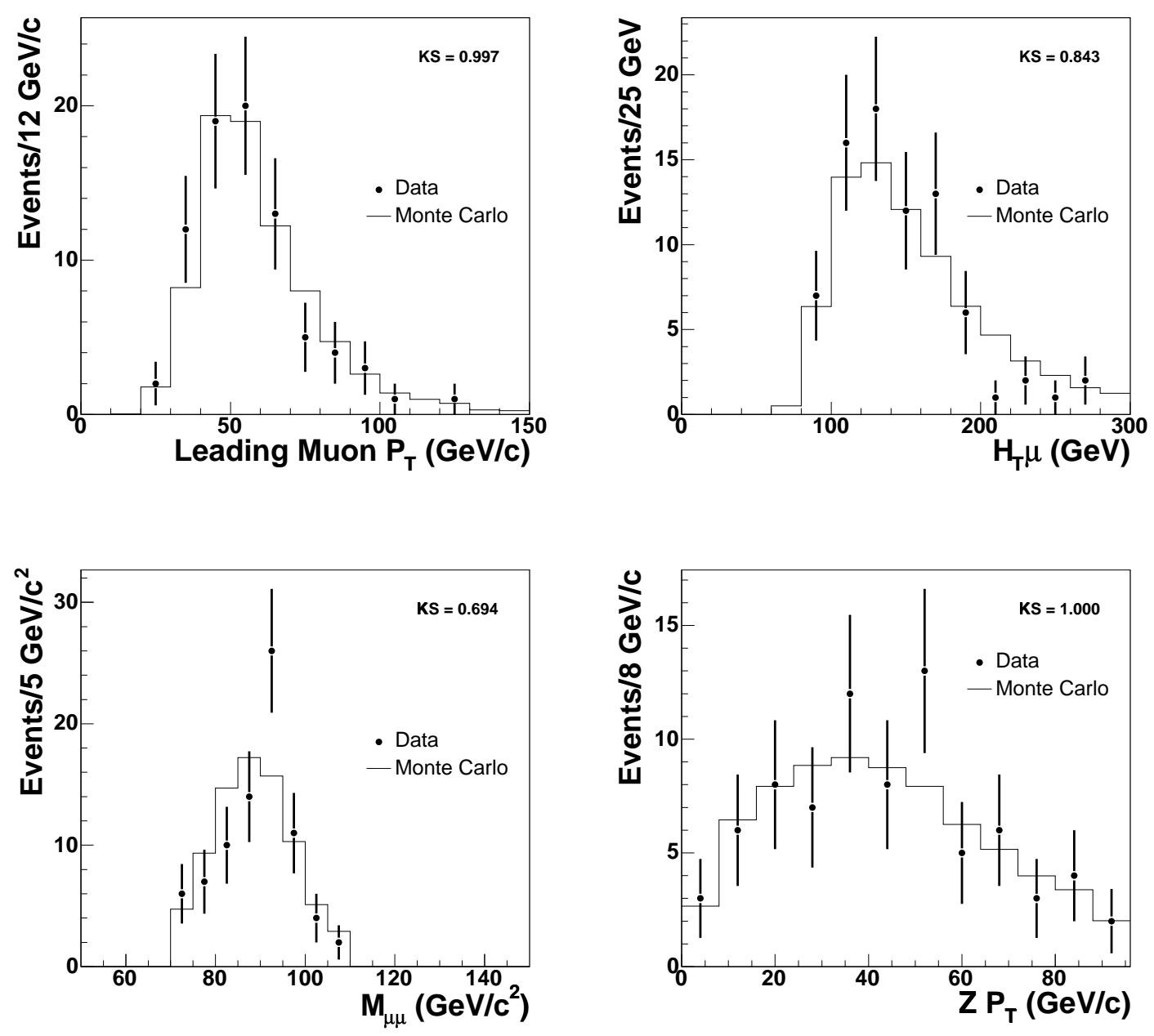

Figure 8.3. Leading muon $\mathrm{P}_{\mathrm{T}}, \mathrm{H}_{T} \mu$, dimuon invariant mass, and the $\mathrm{Z}_{\mathrm{T}}$ spectrum for $\mathrm{Z}$ control events in data and Monte Carlo. 


\begin{tabular}{|l|c|c|c|c|}
\hline MC Sample & $\begin{array}{c}\text { Total MC } \\
\text { Events }\end{array}$ & $\begin{array}{c}\text { Events Passing } \\
\text { All Cuts }\end{array}$ & $\sigma \times B R(p b)$ & Normalized Yield \\
\hline $\mathrm{Z} / \gamma^{*}$ mass1 & 122400 & 57 & 6.27 & 0.32 \\
$\mathrm{Z} / \gamma^{*}$ mass2 & 144600 & 77 & 26.7 & 1.55 \\
$\mathrm{Z} / \gamma^{*}$ mass3 & 95900 & 526 & 0.29 & 0.17 \\
\hline & & \multicolumn{2}{|c|}{ Total } & $\mathbf{2 . 0 4} \pm \mathbf{0 . 3 8}$ (stat.) \\
\hline
\end{tabular}

TABLE 8.3. Estimated $\mathrm{Z} / \gamma^{*}$ yields from Monte Carlo, scaled to data. The data/Monte Carlo normalization factor is 0.781 .

\begin{tabular}{|c|c|c|c|c|}
\hline JES & Raw $Z / \gamma^{*}$ Yield & Scale Factor & Expected Yield & $\Delta$ \\
\hline Default JES & 2.616 & 0.781 & 2.044 & - \\
JES $+1 \sigma$ & 3.953 & 0.595 & 2.352 & +0.308 \\
JES $-1 \sigma$ & 1.959 & 1.004 & 1.967 & -0.077 \\
\hline
\end{tabular}

TABLE 8.4. Z $/ \gamma^{*}$ yields for the default Jet Energy Scale, and with the JES increased and decreased by $1 \sigma$.

mass ranges to the $\mathrm{Z} / \gamma^{*}$ background estimate.

The affect of the Jet Energy Scale (JES) on the expected Z/ $\gamma^{*}$ yield was studied using the JES errors described in Section 6.5.2. The $\mathrm{E}_{\mathrm{T}}$ of the Monte Carlo jets was shifted by the error on the JES and the expected yields were recalculated. The data/Monte Carlo scale factor was also recalculated. Table 8.4 summarizes the results.

\subsubsection{Z $/ \gamma^{*}$ Reweighting}

The jets in the $\mathrm{Z}$ control sample in data tend to have less $\mathrm{E}_{\mathrm{T}}$ than those in Monte Carlo events, as shown in Figure 8.2. To determine the effect of this on the estimated $\mathrm{Z} / \gamma^{*}$ background, a reweighting study was done. A Monte Carlo event weight based on the leading and second jet $\mathrm{E}_{\mathrm{T}}$ spectrum was derived by taking the ratio of the data and Monte Carlo distributions for the jet $\mathrm{E}_{\mathrm{T}}$ in bins of $10 \mathrm{GeV}$. Bins with less than five data events were ignored and the weight for these bins was set to one. The total event weight is the product of the weight from the leading jet and the weight 

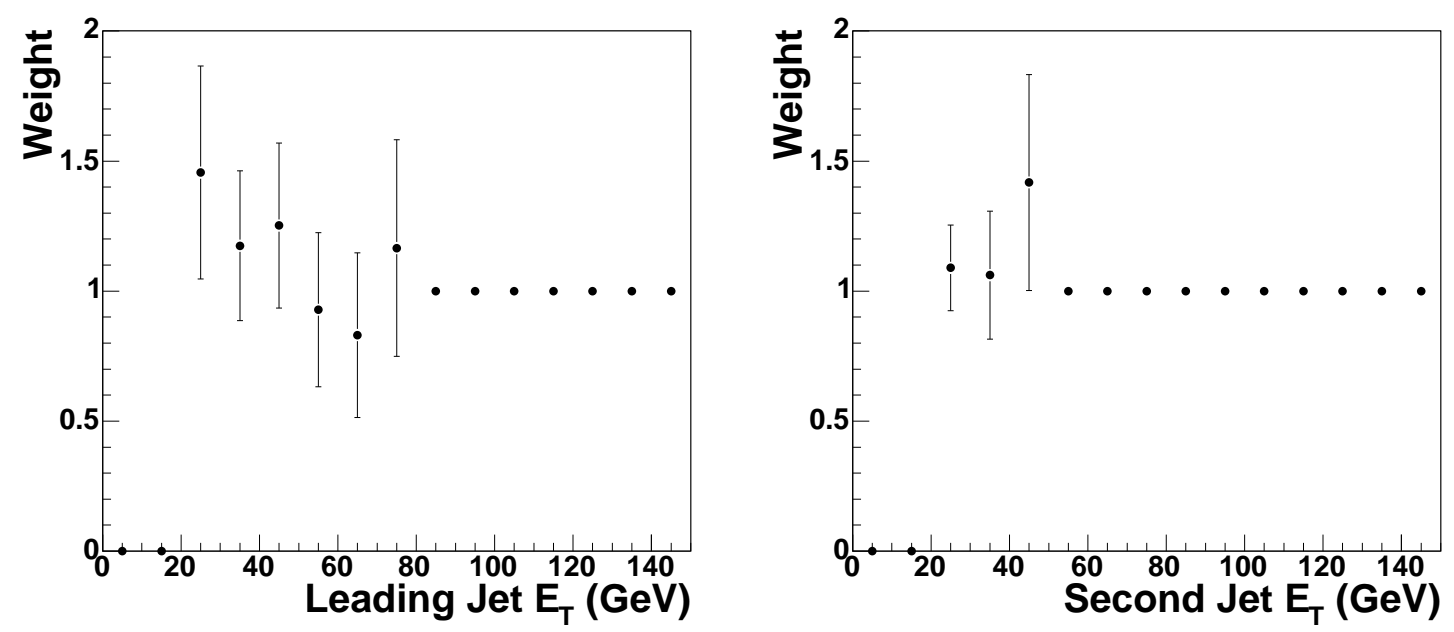

Figure 8.4. The event weights for the leading and second jet as a function of the jet $\mathrm{E}_{\mathrm{T}}$.

\begin{tabular}{|c|c|c|c|c|}
\hline $\begin{array}{c}\text { Monte Carlo } \\
\text { Events Passing } \\
\text { (Reweighted) }\end{array}$ & $\begin{array}{c}\text { Monte Carlo } \\
\sigma x B R(p b)\end{array}$ & $\begin{array}{c}\text { Monte Carlo } \\
\text { Yield } \\
\text { (Reweighted) }\end{array}$ & Data Events & $\begin{array}{c}\text { Scale Factor } \\
\kappa_{Z} \\
\text { (Reweighted) }\end{array}$ \\
\hline 4857.86 & 26.7 & 125.4 & 80 & $\mathbf{0 . 6 3 8} \pm \mathbf{0 . 0 7 2}$ \\
\hline
\end{tabular}

TABLE 8.5. The reweighted scale factor from the $\mathrm{Z}$ control sample.

from the second jet. Figure 8.4 shows the weights for the two jet spectra.

The difference (-0.22 events) in the reweighted yield is taken as a systematic uncertainty on the background. The final estimate for the $\mathrm{Z} / \gamma^{*}$ background is

$$
N_{Z / \gamma^{*}}=2.04 \pm 0.38\left(\text { stat }{ }_{-0.23}^{+0.31}\right. \text { (sys) events }
$$

where the systematic error is a combination of the JES error and the modeling error added in quadrature. No efficiency systematic was calculated for the $\mathrm{Z} / \gamma^{*}$ background because the Monte Carlo prediction is normalized to the data. Variations in the efficiencies would affect the scale factor as well as the Monte Carlo prediction, and the effects would cancel. 


\begin{tabular}{|c|c|c|c|}
\hline MC Sample & Unweighted Events & Reweighted Events & Reweighted Yield \\
\hline $\mathrm{Z} / \gamma^{*}$ mass1 & 57 & 62.0 & 0.28 \\
$\mathrm{Z} / \gamma^{*}$ mass2 & 77 & 83.8 & 1.38 \\
$\mathrm{Z} / \gamma^{*}$ mass3 & 526 & 600.3 & 0.16 \\
\hline & & Total & $\mathbf{1 . 8 2}$ \\
\hline
\end{tabular}

TABLE 8.6. Estimated reweighted $\mathrm{Z} / \gamma^{*}$ yields from Monte Carlo, scaled to data. The reweighted data/Monte Carlo normalization factor is 0.638 .

\begin{tabular}{|l|c|c|c|c|}
\hline MC Sample & $\begin{array}{c}\text { Total MC } \\
\text { Events }\end{array}$ & $\begin{array}{c}\text { Events Passing } \\
\text { All Cuts }\end{array}$ & $\sigma \times B R(\mathrm{pb})$ & Yield \\
\hline WW & 19500 & 50 & 0.29 & 0.104 \\
\hline WW (JES +1) & 19500 & 66 & 0.29 & 0.137 \\
\hline WW (JES -1) & 19500 & 39 & 0.29 & 0.081 \\
\hline & & Total & $\mathbf{0 . 1 0} \pm \mathbf{0 . 0 2}$ \\
\hline
\end{tabular}

TABLE 8.7. Estimated WW yields from Monte Carlo for the default jet energy scale and with the jet energy scale increased and decreased by one sigma.

\subsection{WW}

The WW background was determined from Monte Carlo events (Section 6.9). While the final state is very similar to top, the relatively low production cross section times branching fraction $(0.044 \mathrm{pb})$ for dimuons gives a small estimated contribution. Fewer events pass the jet cuts compared to signal events as well because the associated jets tend to have less energy than the jets from b quarks in $t \bar{t}$ events. Fifty Monte Carlo events pass all cuts, giving an estimated contribution of $0.10 \pm 0.02$ (stat) events. Table 8.7 gives a summary of this background.

The systematic errors on the signal efficiency (Table 7.4) were used for the WW background, except for the Jet Energy Scale error that was determined specifically for the WW background. The total efficiency systematic for the signal (without the JES errors) is $12 \%$, which gives a systematic error due to the efficiency of 0.01 events. The JES errors were determined in the same manner as the signal and $\mathrm{Z} / \gamma^{*}$ sample 
by allowing the $\mathrm{E}_{\mathrm{T}}$ of the Monte Carlo jets to vary and determining the number of Monte Carlo events passing all cuts. The results for the JES errors are shown in Table 8.7. The final result for the $\mathrm{WW}+2$ jet background is

$$
N_{W W}=0.10 \pm 0.02 \text { (stat.) } \pm 0.03 \text { (sys.) events }
$$

where the systematic error is a combination of the efficiency systematic and the JES systematic errors.

\subsection{WQCD Backgrounds}

Muons arising from the decay of heavy quarks are produced in association with jets (from the quark hadronization) and in general do not pass the muon isolation requirements. Some fraction of these muons, however, can fake the isolation requirement and pass the selection criteria. The main sources of these events are $\mathrm{W}+$ jets events, where there is one real isolated muon from the $\mathrm{W}$ boson decay and one muon from heavy flavor decay. In addition, backgrounds can arise from $c \bar{c}$ and $b \bar{b}$ events, where two muons in jets pass the isolation requirements. These backgrounds are collectively called WQCD and are measured using data. These events have at most one real isolated muon (in the case of $\mathrm{W}+$ jets events) and therefore one or two non-isolated muons must pass the isolation requirement.

The determination of the amount of WQCD events in the data was performed using the matrix method. The matrix method uses two samples, a tight sample with all cuts applied, and a loose sample with the cut in question (muon isolation) relaxed. These two samples are combined with the efficiency for signal and background events to pass the muon isolation cut to solve for the amount of signal and background in the tight data sample. In this case, signal refers to all events with two isolated muons (signal, Z/ $\gamma^{*}$, and WW events) and background refers to the WQCD events. 


\subsubsection{WQCD Isolation Efficiency}

The efficiency for a non-isolated muon to 'fake' isolation is measured from data. Events are required to have two jets with $\mathrm{E}_{\mathrm{T}}>20 \mathrm{GeV}$. Additionally, the scaler sum of the $\mathrm{E}_{\mathrm{T}}$ of all jets in the event $\left(\mathrm{H}_{T} 25\right)$ is required to be greater than $100 \mathrm{GeV}$. The efficiency is defined as

$$
\epsilon_{W Q C D}=\frac{\text { Isolated muons with } P_{T}>15 \mathrm{GeV}}{\text { All muons with } P_{T}>15 \mathrm{GeV}}
$$

The efficiency is plotted as a function of the $E_{T}$ in the event (Figure 8.5). A fit is performed for small values of $E_{T}(<25 \mathrm{GeV})$, where background events are expected to dominate the sample. The resulting efficiency for WQCD events to pass the isolation requirement is

$$
\epsilon_{W Q C D}=0.049 \pm 0.004 \text { (stat.) }
$$

The efficiency for signal muons to pass the isolation requirement is $81.5 \%$ per muon (measured from signal Monte Carlo, Section 7.2). A data/Monte Carlo correction factor of .926 per muon was measured for the muon isolation cut (Section 7.2.3). The product of these is the corrected efficiency (per muon) for signal muons to pass the isolation requirement and is

$$
\epsilon_{t \bar{t}}=.755 \pm .029(\text { stat.) }
$$

\subsubsection{Matrix Method}

Data were used to measure the number of tight (passing all cuts) and loose (allow one non-isolated muon) events. The tight sample contains four events, while the loose sample has fourteen. These can be expressed as a function of the unknown number 


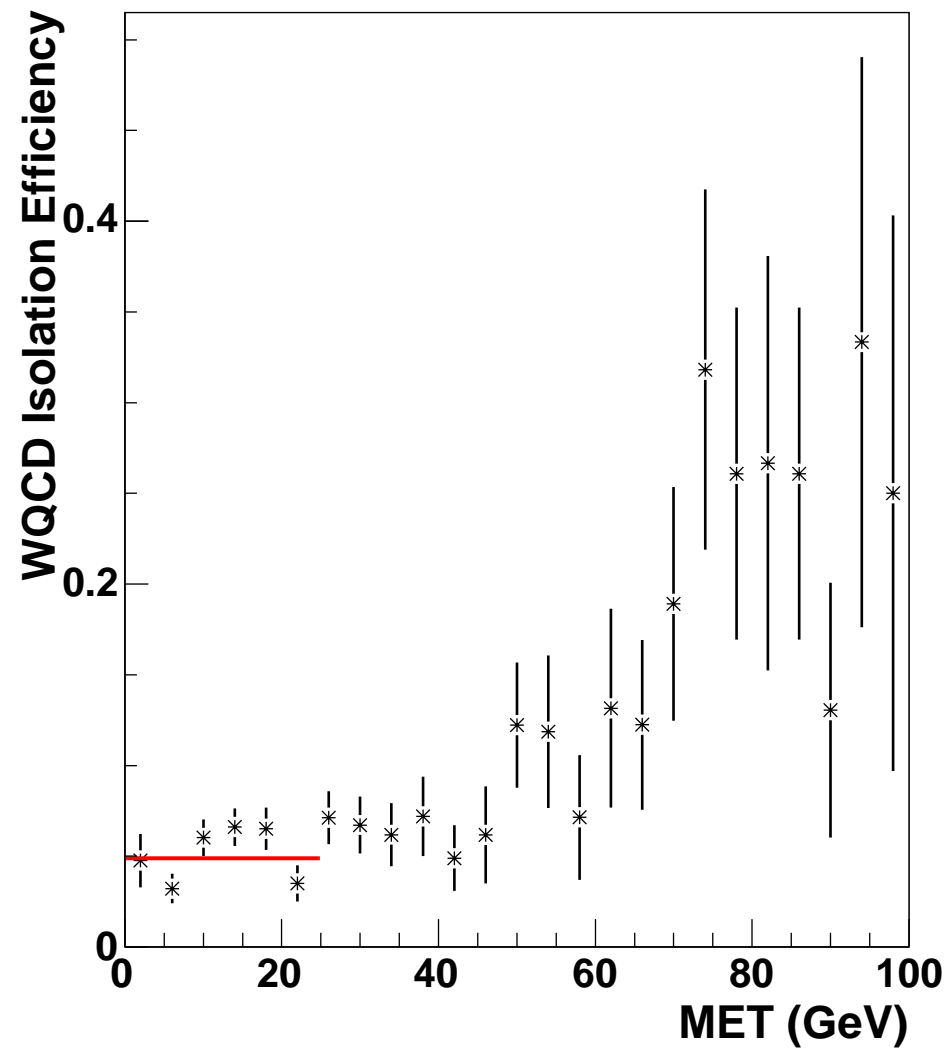

FIgURE 8.5. Isolation efficiency for WQCD events in data as a function of $\not_{T}$. A fit to the low $\not_{T}$ region gives $\epsilon_{W Q C D}=0.049$. 
of signal and background events, $N_{\text {sig }}$ and $N_{W Q C D}$, and the efficiencies for signal and background events to pass the isolation cuts, $\epsilon_{s i g}$ and $\epsilon_{W Q C D}$ :

$$
\begin{aligned}
& N_{l}=N_{\text {sig }}+N_{W Q C D} \\
& N_{t}=\epsilon_{\text {sig }} N_{\text {sig }}+\epsilon_{W Q C D} N_{W Q C D}
\end{aligned}
$$

The substitutions $\mathrm{N}_{1}=\mathrm{N}_{l}-\mathrm{N}_{t}$ and $\mathrm{N}_{2}=\mathrm{N}_{t}$ are used to create two independent variables. In terms of the unknowns $N_{\text {sig }}$ and $N_{W Q C D}$, we then have:

$$
\begin{aligned}
& N_{1}=\left(1-\epsilon_{s i g}\right) N_{s i g}+\left(1-\epsilon_{W Q C D}\right) N_{W Q C D} \\
& N_{2}=\epsilon_{s i g} N_{s i g}+\epsilon_{W Q C D} N_{W Q C D}
\end{aligned}
$$

While the above equations can be solved directly, assignment of the statistical error is difficult with the small data set. Instead, a negative log-likelihood fit is performed numerically to maximize the probability of observing $\mathrm{N}_{1}^{o b s}$ and $\mathrm{N}_{2}^{o b s}$ events by varying the 'average' $\mathrm{N}_{1}$ and $\mathrm{N}_{2}$, given by Equation 8.5. Errors are assigned by varying the fit until the negative log-likelihood has increased by one.

The Poisson probability of observing $\mathrm{N}^{\text {obs }}$ events with an average of $\mathrm{N}$ events is

$$
P=\frac{N^{N^{o b s}}}{N^{o b s !}} e^{N}
$$

and has a maximum when $\mathrm{N}=\mathrm{N}^{o b s}$. The likelihood is defined as the product of the probabilities for observing $\mathrm{N}_{i}$ events, where i runs over the independent variables $\mathrm{N}_{1}$ and $\mathrm{N}_{2}$, defined in Equation 8.5.

$$
\mathcal{L}=\prod_{i} P_{i}=\prod_{i} \frac{N_{i}^{N_{i}^{o b s}}}{N_{i}^{o b s} !} e^{N_{i}}
$$

Here $\mathrm{P}_{i}$ is the Poisson probability for observing $\mathrm{N}_{i}^{\text {obs }}$ events (Equation 8.6) and $\mathrm{N}_{i}$ is given in terms of $\mathrm{N}_{s i g}$ and $\mathrm{N}_{W Q C D}$ in Equation 8.5. 
The likelihood is maximized by varying $\mathrm{N}_{1}$ and $\mathrm{N}_{2}$ given the constraints that $\mathrm{N}_{1}^{o b s}$ has ten events and $\mathrm{N}_{2}^{\text {obs }}$ has four. In practice, two times the negative of the natural logarithm of the likelihood is minimized using MINUIT.

$$
M=-2 \ln (\mathcal{L})=-2\left[N_{1}^{o b s} \ln N_{1}-N_{1}+N_{2}^{o b s} \ln N_{2}-N_{2}\right]
$$

The negative sign is included because MINUIT searches for minima, not maxima. MINOS returns errors by allowing the variables to float until the negative log-likelihood has increased by one. The factor of two allows the errors returned by MINUIT $(\Delta(-$ $2 \cdot \ln \mathcal{L})=+1)$ to correspond to the $1 \sigma(68 \% \mathrm{CI})$ errors.

The systematic error on the WQCD background comes from the uncertainty in the efficiency of the isolation cut for signal and background events. The value of the signal and background efficiencies were varied by their $1 \sigma$ errors and a new fit was performed. Varying the background efficiency by $\pm 1 \sigma$ gave new fit results of 0.50 and 0.42 events ( \pm 0.04 events), while allowing the signal efficiency to vary gave a difference of \pm 0.02 events.

The final result for the WQCD background estimate is

$$
N_{W Q C D}=0.46_{-0.15}^{+0.19} \text { (stat.) } \pm 0.04 \text { (sys.) events }
$$

\section{$8.5 \mathbf{H}_{T} \mu / H_{T}$ Cut Optimization}

To determine the optimal values of the $\mathrm{H}_{T} \mu$ and $\not_{T}$ cut for distinguishing signal from background, a $2 \mathrm{D}$ grid search was performed for a range of $\mathrm{H}_{T} \mu$ and $\mathbb{E}_{T}$ cut values. The figure of merit, $\sigma$, was maximized to find the optimal cut values:

$$
\sigma=\frac{s}{\sqrt{s+b}}
$$

where $\mathrm{s}$ and $\mathrm{b}$ are the expected signal and background yields, respectively. 
ALPGEN Monte Carlo was used to simulate the $t \bar{t}$ signal, and the ALPGEN Z $/ \gamma^{*}$ and WW samples described in Table 6.9 were used for the backgrounds. For each sample, the full set of kinematic cuts was applied, minus the $\mathbb{E}_{T}$ and $\mathrm{H}_{T} \mu$ cuts. A 2D scatter plot was made for $\mathrm{H}_{T} \mu$ as a function of $E_{T}$ for each Monte Carlo sample. These plots were then used to determine the total number of events passing a range of $\mathrm{H}_{T} \mu$ and $E_{T}$ cuts. Figure 8.6 shows the scatter plots for the signal and mass $2 \mathrm{Z} / \gamma^{*}$ background sample, which is the dominant background.
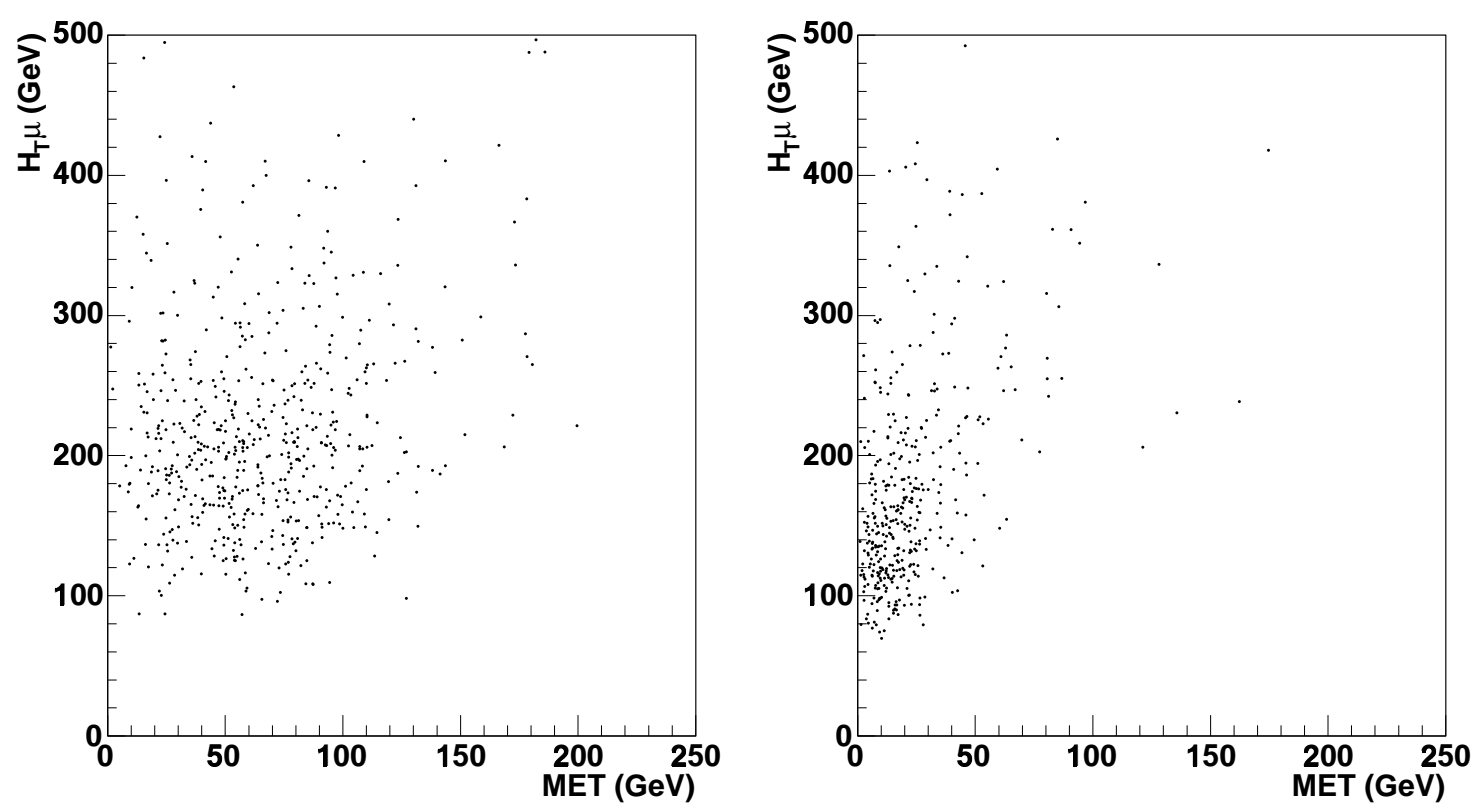

Figure 8.6. Scatter plots for $\mathrm{H}_{T} \mu$ versus $\mathbb{E}_{T}$ for $t \bar{t}$ (left) and $\mathrm{Z} / \gamma^{*}$ mass 2 Monte Carlo (right).

The number of Monte Carlo events passing the different cuts were converted to expected yields via

$$
\text { Yield }=\frac{M C_{\text {Pass }} \times \sigma_{M C} \times \mathcal{L}_{\text {Int }} \times \kappa}{M C_{\text {Total }}}
$$

where $M C_{\text {Pass }}$ is the number of Monte Carlo events that pass all cuts, $M C_{\text {Total }}$ is the 

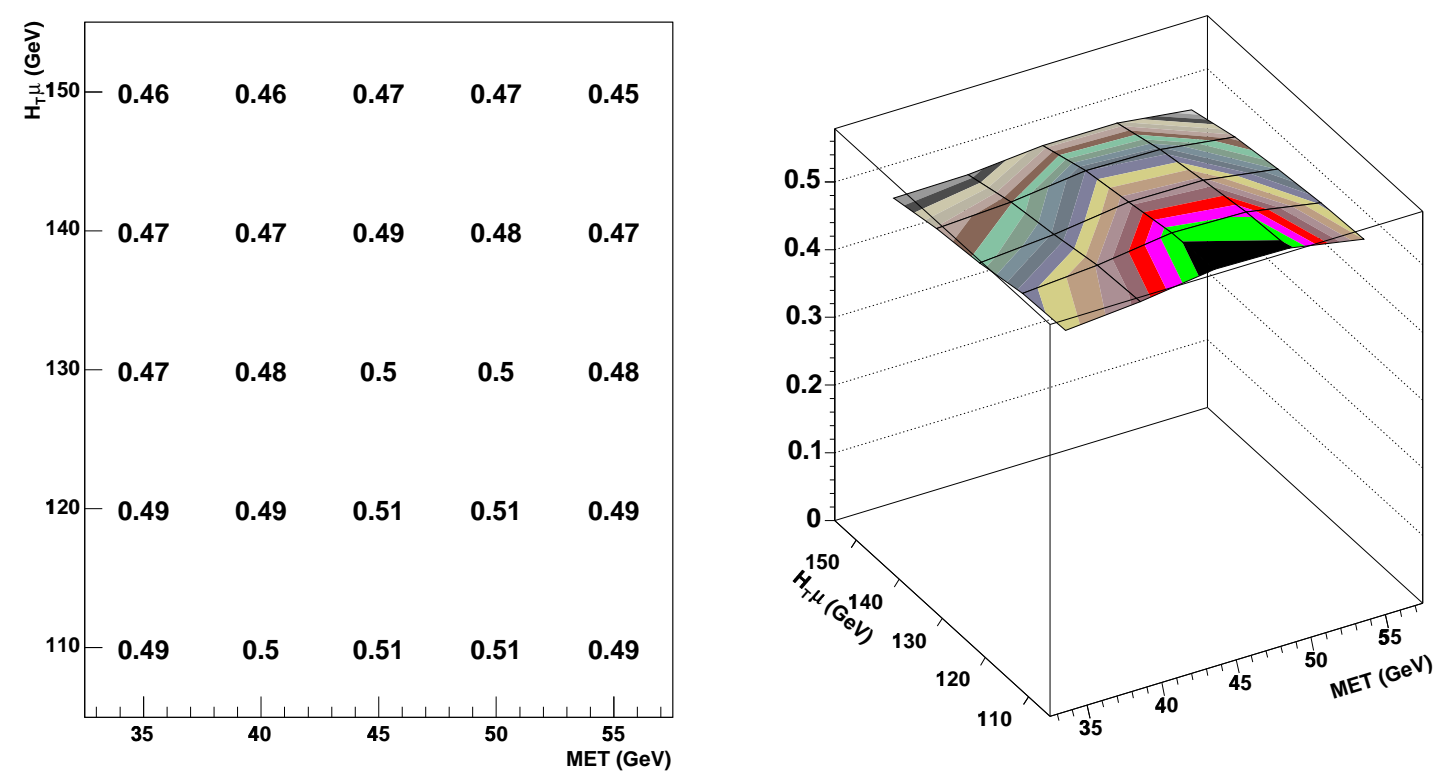

Figure 8.7. Plot of the inverse of the FOM for various values of the $\mathbb{E}_{T}$ and $\mathrm{H}_{T} \mu$ cut. The plot on the left shows the values of the FOM, with the maximum of 0.51 reached at a $E_{T}$ cut of $45 \mathrm{GeV}$ and a $\mathrm{H}_{T} \mu$ cut of $120 \mathrm{GeV}$ (some adjacent values have the same FOM). The plot on the right shows contours for the FOM in the $E_{T^{-}} \mathrm{H}_{T} \mu$ plane.

total number of Monte Carlo events, $\sigma_{M C}$ is the Monte Carlo cross section, $\mathcal{L}_{\text {Int }}$ is the total integrated data luminosity, and $\kappa$ is the data/Monte Carlo scale factor for the different samples (when necessary). For the $\mathrm{Z} / \gamma^{*}$ events, the scale factor is 0.781 (Section 8.2.2). For the $t \bar{t}$ signal Monte Carlo, the scale factor is 0.828 , the product of the four derived data/Monte Carlo factors (Table 7.1). No data/Monte Carlo factor was derived for the WW Monte Carlo and it was taken as one for this study.

The grid search (Figure 8.7) shows the optimal cut value of $E_{T}>45 \mathrm{GeV}$ and an optimal $\mathrm{H}_{T} \mu$ cut of $120 \mathrm{GeV}$ or less. In $\mathrm{H}_{T} \mu$, the optimal peak begins at $120 \mathrm{GeV}$ and is constant for all values of $\mathrm{H}_{T} \mu<120 \mathrm{GeV}$. While the $\mathrm{Z} / \gamma^{*}$ events do tend to have a lower value for $\mathrm{H}_{T} \mu$ than the signal (Figure 8.6), this difference disappears 


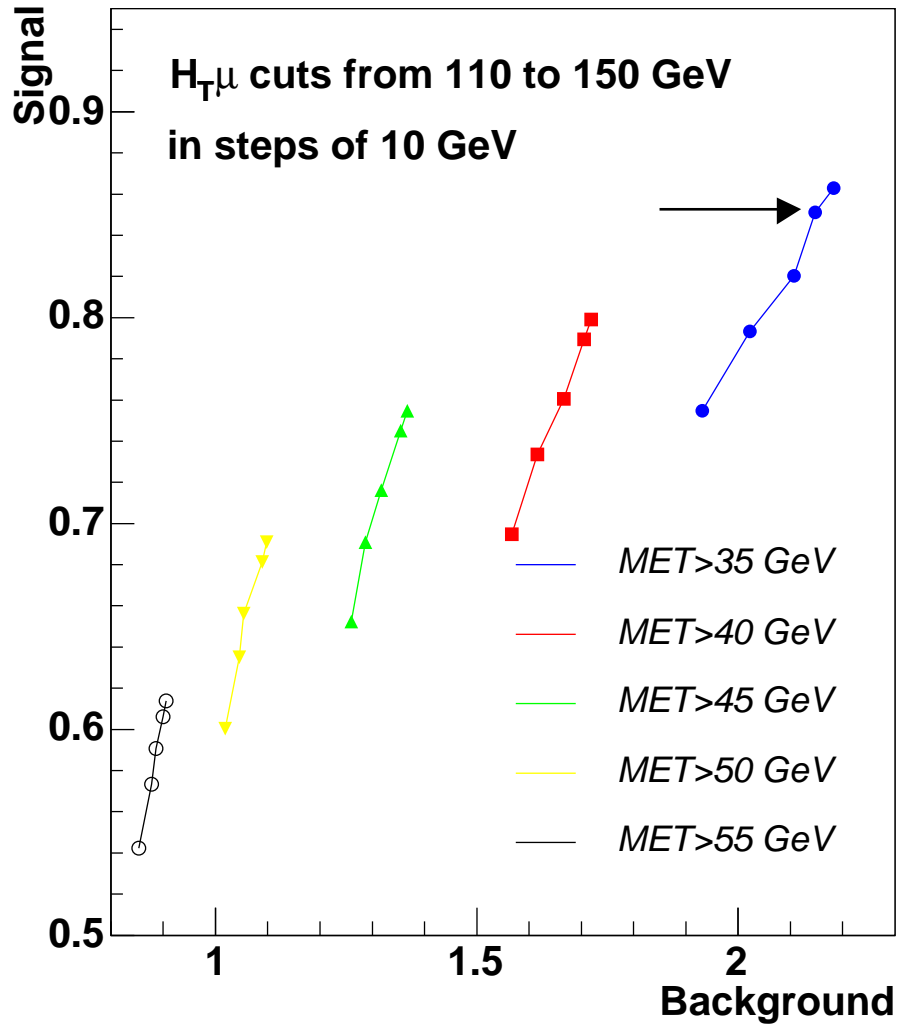

Figure 8.8. Plot of the expected signal yield as a function of the expected background $\left(\mathrm{Z} / \gamma^{*}\right.$ and $\mathrm{WW}$ events) yield for various values of the $\mathbb{E}_{T}$ and $\mathrm{H}_{T} \mu$ cut. The chosen cut of $E_{T}>35 \mathrm{GeV}$ and $\mathrm{H}_{T} \mu>120 \mathrm{GeV}$ is shown with the arrow. 
once an $\mathbb{E}_{T}>35 \mathrm{GeV}$ cut is applied. As a sanity check, the optimization was also done with all data/Monte Carlo scale factors set to one. The location of the maxima did not change, only the absolute value of the maximum for the FOM.

Though the optimization shows a preferred cut of $E_{T}>45 \mathrm{GeV}$, this analysis used a cut of $E_{T}>35 \mathrm{GeV}$ to be consistent with the other dilepton analysis. A cut of $\mathrm{H}_{T} \mu>120 \mathrm{GeV}$ was selected as the tightest cut in $\mathrm{H}_{T} \mu$ at the maximum value of the FOM.

\subsection{Data/Monte Carlo Comparisons}

In order to compare the predicted background yields to the data, the selection cuts $\left(\Delta \phi\left(\mu_{\text {leading }}, \mathbb{E}_{T}\right), \mathrm{M}_{\mu \mu}, \mathrm{H}_{T} \mu\right.$, and $\left.\mathbb{E}_{T}\right)$ were removed to give a data set dominated by background that could be compared with Monte Carlo predictions. With only the preselection cuts, the data contains 128 events, while the Monte Carlo prediction is roughly 132 background events and two signal events. Of the 132 predicted background events, 124 are expected to be $\mathrm{Z} / \gamma^{*}$ events.

Figures 8.9 - 8.11 show several kinematic distributions comparing Monte Carlo expectations from signal and background (histograms) and the observed data events (points) after preselection cuts have been applied. The dominant $\mathrm{Z} / \gamma^{*}$ background is shown in light gray. Figure 8.9 shows the leading muon $\mathrm{P}_{\mathrm{T}}$ and $|\eta|$. Figure 8.10 shows the leading jet $\mathrm{E}_{\mathrm{T}}$ and $\mathrm{H}_{T} \mu$. Finally, Figure 8.11 shows the invariant mass and the $\mathbb{F}_{T}$ spectrum, with arrows indicating the selection cuts. Good agreement is seen in the plots with the exception of the leading jet $\mathrm{E}_{\mathrm{T}}$ distribution, where the data events tend to have less $\mathrm{E}_{\mathrm{T}}$ than the Monte Carlo. This is the same result that was seen in the $\mathrm{Z}$ control sample that led to a reweighting study (Section 8.2.4). Since all 80 of the $\mathrm{Z}$ control data events are also in the preselected data sample of 128 events, this result is not surprising. 

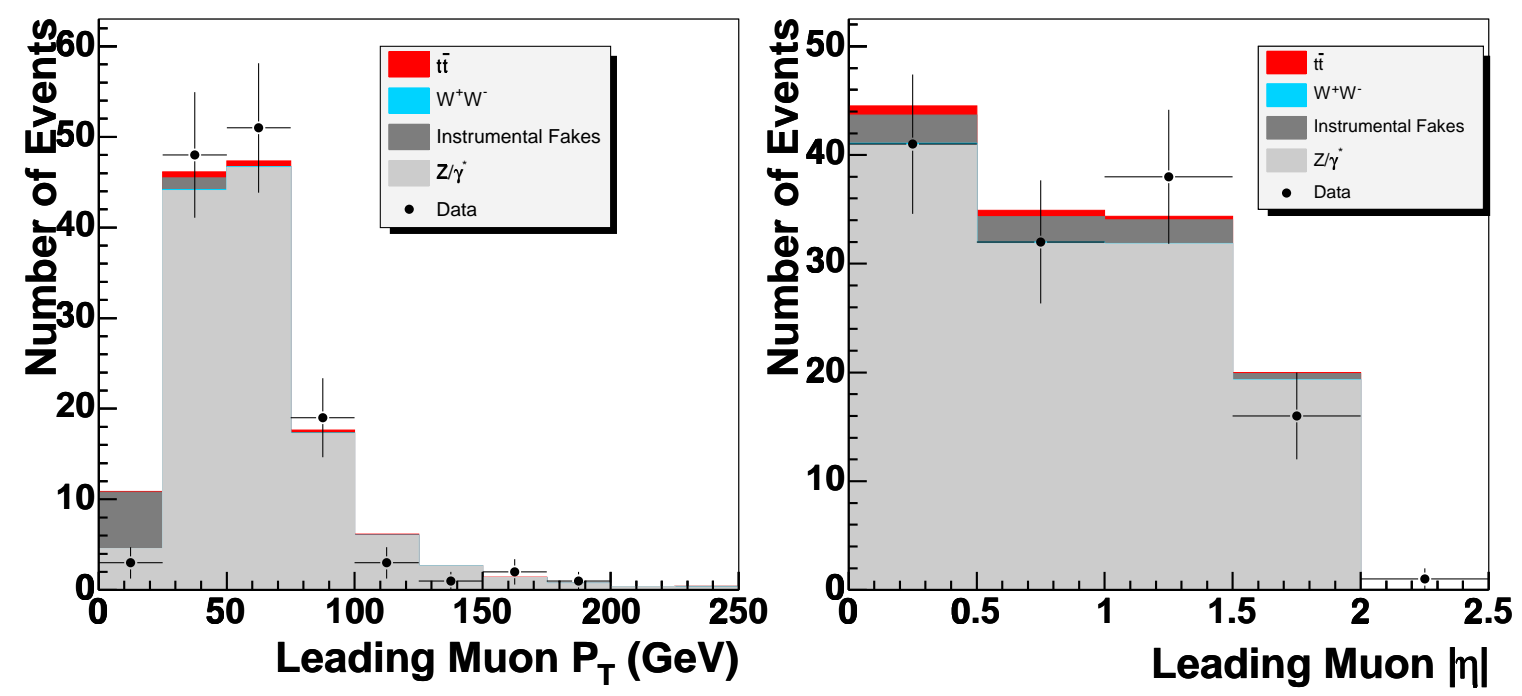

Figure 8.9. Leading muon $\mathrm{P}_{\mathrm{T}}$ (left) and $|\eta|$ (right).
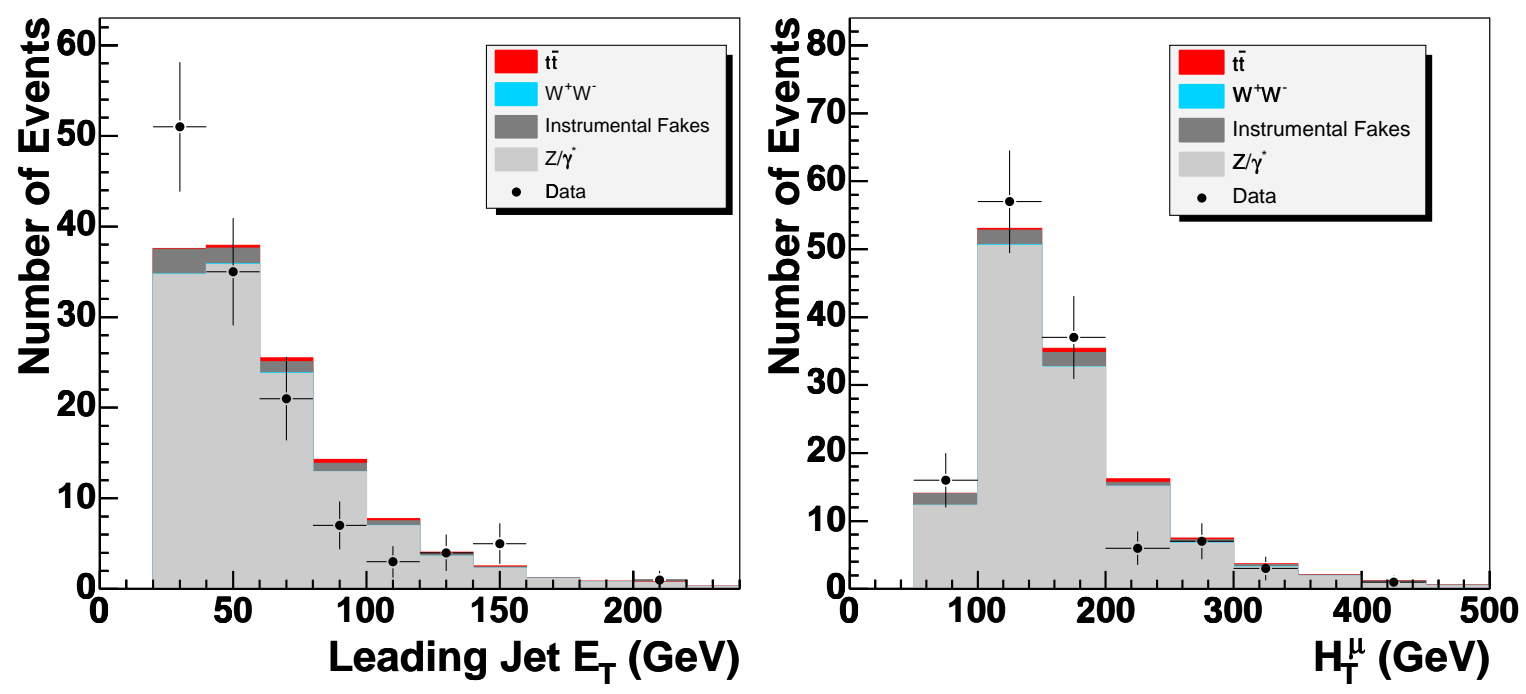

FiguRE 8.10. Leading jet $\mathrm{E}_{\mathrm{T}}$ (right) and $\mathrm{H}_{T} \mu$ (left). 

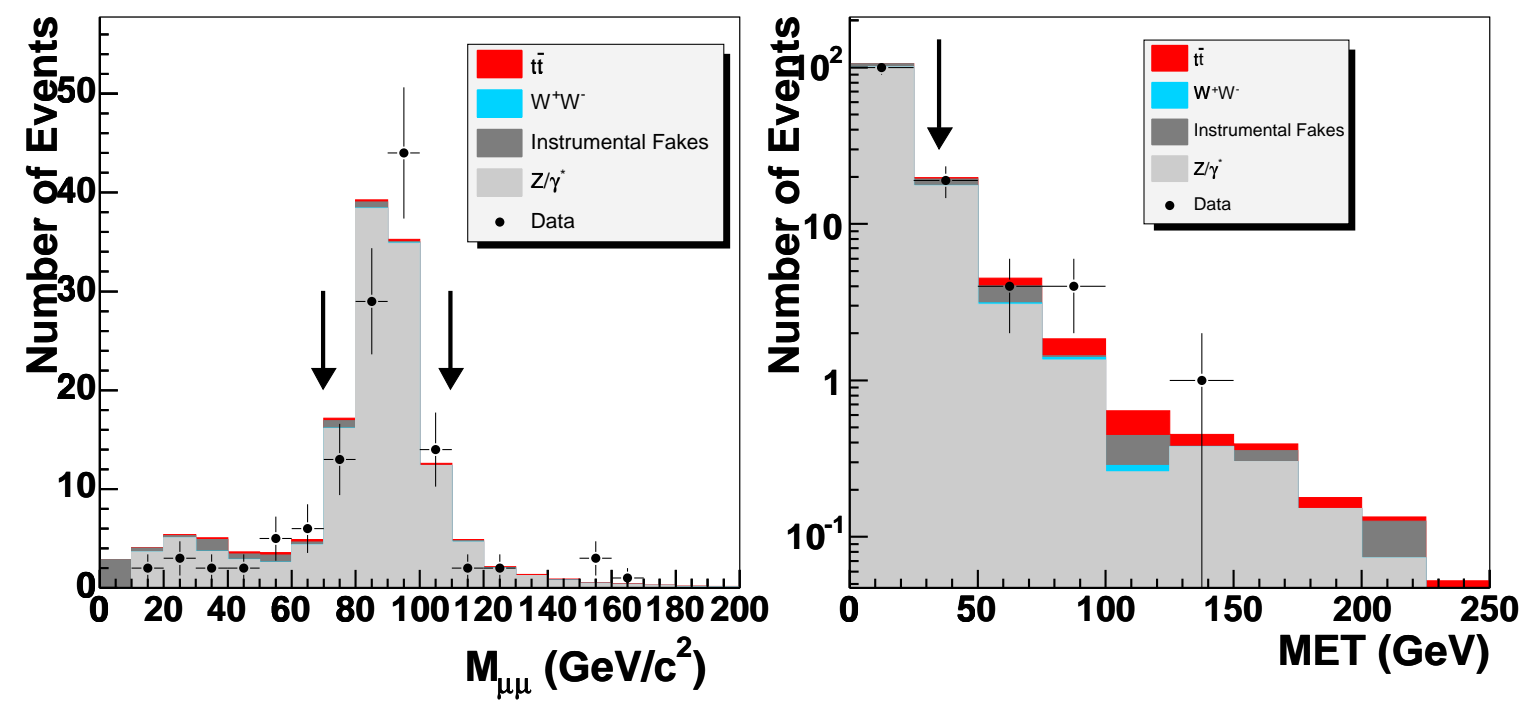

Figure 8.11. Dimuon invariant mass (left) and $E_{T}$ (right). The arrows indicate the selection cuts.

\subsection{Background Summary}

Table 8.8 summarizes the backgrounds evaluated for this analysis, while Table 8.9 summarizes the sources of systematic errors for each background. $\mathrm{Z} / \gamma^{*}$ events are the dominant background, accounting for roughly $80 \%$ of the expected 2.6 background events. The large uncertainty on this background also leads to the largest systematic uncertainty on the final cross section result (Section 9.2). Using a likelihood fitter or a neural net could reduce the expected background by using a combination of topological variables to distinguish between signal and background events. Improvements could also be made by rejecting $\mathrm{Z} / \gamma^{*}$ events with poorly reconstructed muons, which tend to pass both the $\mathbb{E}_{T}$ and $\mathrm{M}_{\mu \mu}$ cuts. Finally, a measurement of the $\mathrm{Z} / \gamma^{*}$ background from data alone would provide an important cross-check for the current estimation. 


\begin{tabular}{|c|c|c|c|c|c|}
\hline & Events & $\begin{array}{c}\text { Stat. } \\
\text { Error }\end{array}$ & $\begin{array}{c}\text { Sys. } \\
\text { Error }\end{array}$ & $\begin{array}{c}\text { JES } \\
\text { Error }\end{array}$ & $\begin{array}{c}\text { Total Sys. } \\
\text { Error }\end{array}$ \\
\hline $\mathrm{Z} / \gamma^{*}$ & 2.04 & \pm 0.38 & ${ }_{-0.22}^{+0.0}$ & ${ }_{-0.08}^{+0.31}$ & ${ }_{-0.23}^{+0.31}$ \\
\hline $\mathrm{WW}$ & 0.10 & \pm 0.02 & \pm 0.01 & ${ }_{-0.02}^{+0.03}$ & \pm 0.03 \\
\hline WQCD & 0.46 & ${ }_{-0.15}^{+0.19}$ & \pm 0.04 & \pm 0.0 & \pm 0.04 \\
\hline Total & $\mathbf{2 . 6 1}$ & ${ }_{-\mathbf{0 . 4 1}}^{+0.42}$ & ${ }_{-\mathbf{0 . 2 2}}^{+0.05}$ & ${ }_{-\mathbf{0 . 0 8}}^{+0.31}$ & ${ }_{-\mathbf{0 . 2 4}}^{+0.31}$ \\
\hline
\end{tabular}

TABLE 8.8. Statistical and systematic errors on all terms in the calculation.

\begin{tabular}{|l||c|l|l|}
\hline Parameter & Fraction & Source & Section \\
\hline $\mathrm{Z} / \gamma^{*}$ JES & $+15-4 \%$ & JES macro & 8.2 .3 \\
$\mathrm{Z} / \gamma^{*}$ Simulation & $-11 \%$ & Re-weighting study & 8.2 .4 \\
WW Efficiency & $+10-12 \%$ & Signal Error (no JES) & 8.3 \\
WW JES & $+32-22 \%$ & JES macro & 8.3 \\
Fake & $10 \%$ & Efficiency errors & 8.4 \\
\hline Total & $\mathbf{+ 1 2 - 9 \%}$ & & \\
\hline
\end{tabular}

TABLE 8.9. Summary of the relative systematic uncertainties on the backgrounds. The total error is from the propagation of all systematic errors on the total background (Table 8.8). 


\section{CHAPTER 9}

\section{RESULTS}

\subsection{Cross Section Result}

The cross section, $\sigma_{t \bar{t}}$, is given by

$$
\sigma_{t \bar{t}}=\frac{\left(N_{o b s}-N_{b k g}\right)}{\epsilon_{s i g} \times B R\left(t \bar{t} \rightarrow \mu^{+} \mu^{-}\right) \times \mathcal{L}_{\text {Int }}}
$$

where the values for the various parameters are summarized in Table 9.1. The value for the branching ratio $\operatorname{BR}\left(t \bar{t} \rightarrow \mu^{+} \mu^{-}\right)$includes the cascade $\mathrm{W} \rightarrow \tau \nu, \tau \rightarrow \mu \nu \nu$. The values for the branching ratios were taken from the 2004 Particle Data Group 'Review of Particle Physics' [13]. A numerical log-likelihood fit is performed to find the cross section and the associated $1 \sigma$ errors. In this case, the number of observed events, $\mathrm{N}_{o b s}$ is four, and the function that is minimized is

$$
M=-2\left[N_{\text {obs }} \ln (N)-N\right]
$$

where $\mathrm{N}$ is the mean number of expected events as a function of $\sigma_{t \bar{t}}$ obtained by re-arranging equation 9.1 .

$$
N=N_{\text {sig }}+N_{\text {back }}=\sigma_{t \bar{t}} \times \epsilon_{\text {sig }} \times B R\left(t \bar{t} \rightarrow \mu^{+} \mu^{-}\right) \times \mathcal{L}_{\text {Int }}+N_{\text {back }}
$$

The cross section, $\sigma_{t \bar{t}}$, is varied to find the minimum of the negative log-likelihood function (Equation 9.2). The $1 \sigma$ errors are the values of $\sigma_{t \bar{t}}$ for which the log-likelihood

\begin{tabular}{|l|l|l|l|l|}
\hline$N_{\text {obs }}$ & $N_{\text {Bkg }}$ & $\epsilon_{\text {sig }}$ & $B R\left(t \bar{t} \rightarrow \mu^{+} \mu^{-}\right)$ & $\mathcal{L}_{\text {Int }}\left(p b^{-1}\right)$ \\
\hline 4 & 2.61 & 0.0569 & 0.01581 & 139.58 \\
\hline
\end{tabular}

TABLE 9.1. Final cross section parameters and result. 


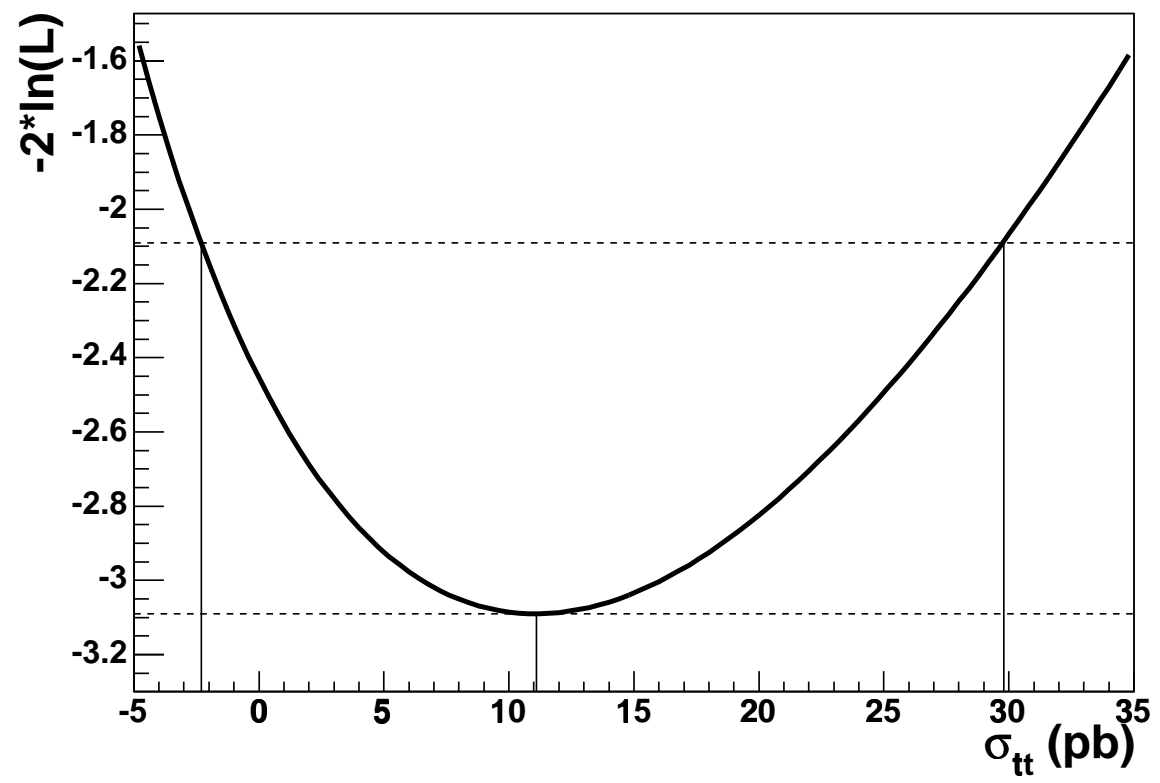

FigurE 9.1. The negative log-likelihood function for the cross section result.

function has increased by one. The result is

$$
\sigma_{t \bar{t}}=11.1_{-13.4}^{+18.7} \text { (stat.) } \mathrm{pb}
$$

where the errors are the statistical errors from the fit (Figure 9.1).

The errors from the log-likelihood fit extend into the non-physical region (negative cross section). The approach of Feldman and Cousins for counting experiments with low statistics is used to determine the range of $\mathrm{N}_{\text {sig }}$, and thus the cross section, within a given confidence interval (CI) given a measured number of events $\mathrm{N}$ and expected background $\mathrm{N}_{b k g}[56]$.

This approach finds all values of $\mathrm{N}$, the number of observed data events, that are consistent within a given CI with the number of background events and the assumed average number of signal events, $\mathrm{N}_{\text {sig }}$. The Poisson probability for a given $\mathrm{N}_{s i g}$ is

$$
P\left(N, N_{s i g}+N_{b k g}\right)=\frac{\left(N_{s i g}+N_{b k g}\right)^{N}}{N !} e^{-\left(N_{s i g}+N_{b k g}\right)}
$$


The approach is:

1. For a given $\mathrm{N}_{\text {sig }}$, find $P\left(N, N_{\text {sig }}+N_{b k g}\right)$ for all reasonable values of the number of data events $\mathrm{N}$ (0 to 19 in this analysis).

2. Also calculate the probability $P\left(N, \mu_{\text {Best }}+N_{b k g}\right)$, where $\mu_{\text {Best }}$ is the larger of $\left(0, N-N_{b k g}\right)$.

3. Calculate the ratio $R=P\left(N, N_{s i g}+N_{b k g}\right) / P\left(N, \mu_{B e s t}+N_{b k g}\right)$.

4. Rank the values of $\mathrm{N}$ by decreasing $\mathrm{R}$.

5. Include all values of $\mathrm{N}$ until the sum of $P\left(N, N_{\text {sig }}+N_{b k g}\right)$ is greater than or equal to the desired confidence interval.

Thus, for a given assumed average number of signal events, there will be a range of the number of data events that fall within the desired CI. This process is repeated for increments of $\mathrm{N}_{\text {sig }}$ of 0.001 . The lower limit for a given CI is the lowest value of $\mathrm{N}_{\text {sig }}$ that includes the number of observed number of data events $\mathrm{N}$, while the upper limit is the highest value of $\mathrm{N}_{\text {sig }}$ that includes N. For a total number or observed events of four, the $68 \%$ CI range for $\mathrm{N}_{\text {sig }}$ is 0.22 to 4.17 events. Figure 9.2 shows the allowed values of $\mathrm{N}_{\text {sig }}$ for various values of the number of data events $\mathrm{N}$ with a background, $\mathrm{N}_{b k g}$, of 2.61. The central value for $\mathrm{N}_{s i g}$ of $1.39\left(\mathrm{~N}-\mathrm{N}_{b k g}\right)$ for four data events is shown with the $68 \%$ CI error bars.

The conversion from $\mathrm{N}_{\text {sig }}$ to the cross section is

$$
\sigma_{t \bar{t}}=\frac{N_{s i g}}{\epsilon_{s i g} \times B R\left(t \bar{t} \rightarrow \mu^{+} \mu^{-}\right) \times \mathcal{L}_{I n t}}
$$

Using the allowed $68 \%$ CI range of values for $\mathrm{N}_{\text {sig }}$ above gives a cross section of

$$
\sigma_{t \bar{t}}=11.1_{-9.3}^{+22.1} \text { (stat.) } \mathrm{pb}
$$




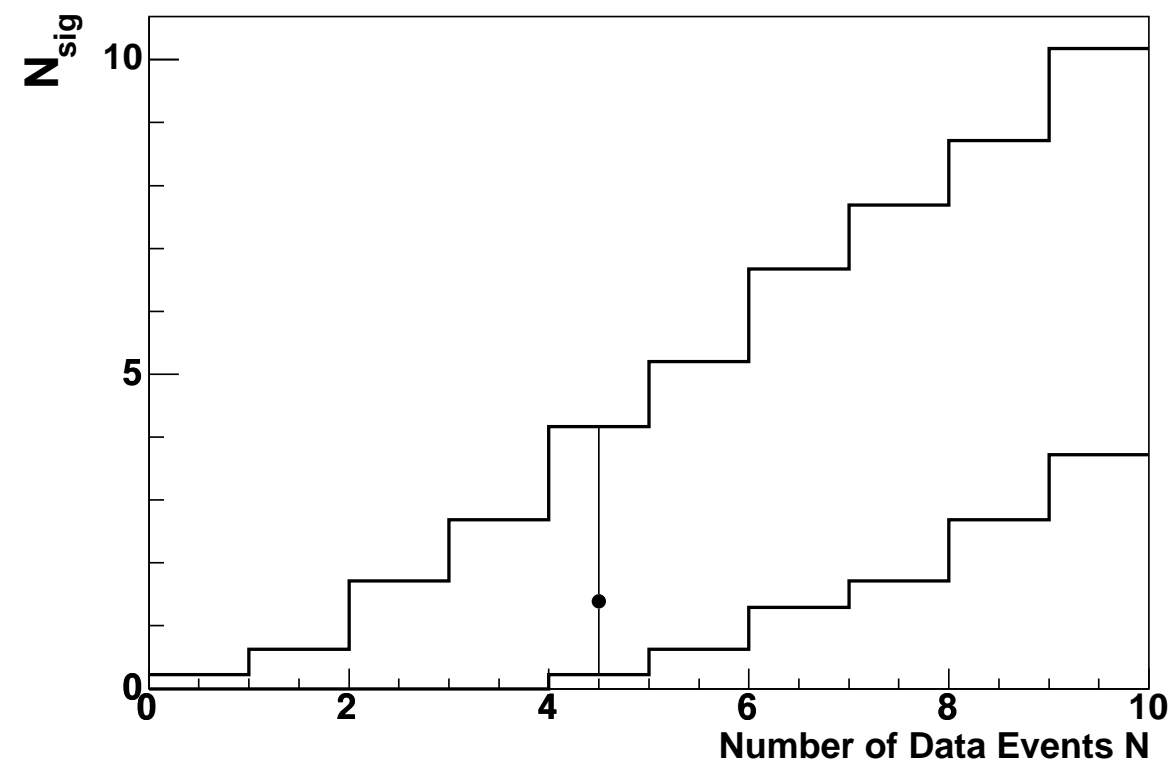

Figure 9.2. The $68 \%$ CI values for $\mathrm{N}_{\text {sig }}$ with $\mathrm{N}_{b k g}$ equal to 2.61 events for various values of $\mathrm{N}$. Also shown is the measured number of data events $\mathrm{N}$ of four.

\subsection{Systematic Errors}

The errors quoted above include only the statistical error associated with a data set of four events. Statistical and systematic errors on the estimated background, the signal efficiency, and the luminosity are included in the systematic error for the cross section. The propagation of errors from these three sources was done in quadrature starting with equation 9.1

$$
\Delta_{\sigma_{t \bar{t}}}=\sqrt{\left(\frac{\partial \sigma_{t \bar{t}}}{\partial N_{b k g}}\right)^{2} \sigma_{N b k g}^{2}+\left(\frac{\partial \sigma_{t \bar{t}}}{\partial \epsilon_{s i g}}\right)^{2} \sigma_{\epsilon s i g}^{2}+\left(\frac{\partial \sigma_{t \bar{t}}}{\partial \mathcal{L}_{I n t}}\right)^{2} \sigma_{\mathcal{L}_{I n t}}^{2}}
$$

where the partial derivatives are equal to 


\begin{tabular}{|l|l|l|l|l|c|}
\hline & Value & $\begin{array}{l}\text { Stat. } \\
\text { Error }\end{array}$ & $\begin{array}{l}\text { Sys. } \\
\text { Error }\end{array}$ & $\begin{array}{l}\text { Total } \\
\text { Error }\end{array}$ & $\begin{array}{c}\text { Error on } \sigma_{t \bar{t}} \\
(p b)\end{array}$ \\
\hline Background (Events) & 2.61 & ${ }_{-0.41}^{+0.42}$ & ${ }_{-0.24}^{+0.31}$ & ${ }_{-0.47}^{+0.53}$ & ${ }_{-4.2}^{+3.7}$ \\
\hline Efficiency & 0.057 & \pm 0.004 & ${ }_{-0.009}^{+0.007}$ & ${ }_{-0.010}^{+0.008}$ & ${ }_{-1.5}^{+1.9}$ \\
\hline Luminosity (pb) & 139.58 & \pm 0.0 & \pm 7.9 & \pm 7.9 & \pm 0.6 \\
\hline & & & & Total & ${ }_{-4.5}^{+4.3}$ \\
\hline
\end{tabular}

TABLE 9.2. Statistical and systematic errors on all terms in the calculation.

$$
\begin{aligned}
\frac{\partial \sigma_{t \bar{t}}}{\partial N_{b k g}} & =-\frac{1}{\epsilon_{s i g} \times B R\left(t \bar{t} \rightarrow \mu^{+} \mu^{-}\right) \times \mathcal{L}_{\text {Int }}} \\
\frac{\partial \sigma_{t \bar{t}}}{\partial \epsilon_{s i g}} & =-\frac{1}{\epsilon_{s i g}} \times \sigma_{t \bar{t}} \\
\frac{\partial \sigma_{t \bar{t}}}{\partial \epsilon_{s i g}} & =-\frac{1}{\mathcal{L}_{\text {Int }}} \times \sigma_{t \bar{t}}
\end{aligned}
$$

Table 9.2 summarizes the errors from these three sources and the contribution to the systematic error on the final cross section result. Table 9.3 gives the individual systematic errors for the signal efficiency. The systematics for the individual background contributions are given in Section 8.7, while the luminosity error is described in Section 6.3. The final cross section result is

$$
\sigma_{t \bar{t}}=11.1_{-9.3}^{+22.1} \text { (stat.) }{ }_{-4.5}^{+4.3} \text { (sys.) } \mathrm{pb}
$$

The next-to-next-to leading order calculation (NNLO) by Kidonakis and Vogt gives a prediction for the $t \bar{t}$ cross section at $\sqrt{s}=1.96 \mathrm{TeV}$ of $6.77 \pm 0.42 \mathrm{pb}$ for $\mathrm{M}_{t}=175 \mathrm{GeV}$ and $\mu=\mathrm{M}_{t}$ (Section 2.2). Figure 9.3 shows the comparison of this measurement with the theoretical value. The solid line is the average NNLO theoretical prediction and the data point is the measured cross section with statistical errors (inner) and total errors (outer). The measured value is consistent with the theoretical value. 


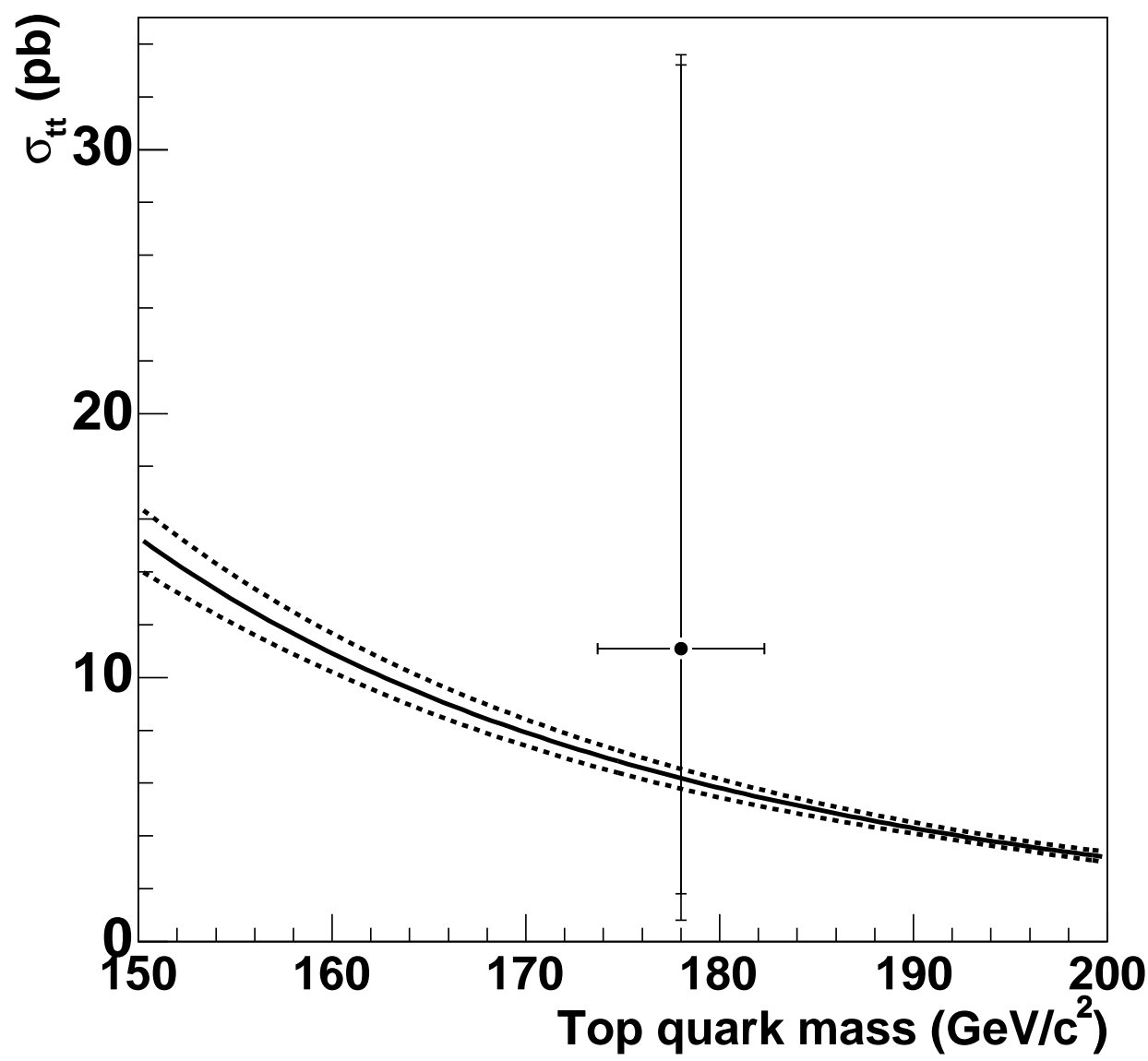

FIgurE 9.3. The theoretical NNLO average cross section (solid line) with errors (dashed line). The data point is the measured value of $11.1 \mathrm{pb}$ at a top mass of 178 $\mathrm{GeV}$. The inner error bar is statistical only, the outer is statistical and systematic combined. 


\begin{tabular}{|c|c|c|c|}
\hline Parameter & Fraction & Source & Section \\
\hline$\mu \mathrm{ID}$ & $3 \%$ & $\mathrm{Z} / \gamma^{*}$ events $\mathrm{w} /$ unbiased muon & 7.2 .1 \\
\hline$\mu$ isolation & $6 \%$ & $\begin{array}{l}\text { Reweighting study } \\
\text { w/signal Monte Carlo }\end{array}$ & 7.2 .3 \\
\hline$\mu$ tracking & $2 \%$ & Data/Monte Carlo tracking macro & 7.2 .2 \\
\hline L1 $\mu$ trigger & $1 \%$ & Trigger macro & 7.3 \\
\hline Jet ID & $-6 \%$ & Reweighted Monte Carlo to reflect data & 7.4 \\
\hline$\mu \sigma_{d c a}$ & $4 \%$ & Statistical error on $\kappa_{D C A}$ & 7.5 \\
\hline JES & $(+6-10) \%$ & JES macro & 7.4 \\
\hline tuneA & $1 \%$ & Alternate Monte Carlo & 7.7 \\
\hline top mass & $6 \%$ & Alternate Monte Carlo & 7.7 \\
\hline Total & $+12-16 \%$ & & \\
\hline No JES & $+10-12 \%$ & & \\
\hline
\end{tabular}

TABLE 9.3. Summary of the relative systematic uncertainties on the $t \bar{t} \rightarrow \mu \mu$ efficiencies. The last row is the total error without the jet energy scale errors, which is used for the WW background.

\subsection{Candidate Events}

A total of four candidate events were found in the data. Table 9.4 summarizes the kinematic properties of the four events. Figures 9.4, 9.5, and 9.6 are event displays for run 178423, event 35258885 and show the muon system, central tracker, and the calorimeter lego display, respectively. In Figure 9.5, the two high $\mathrm{P}_{\mathrm{T}}$ muons are identified by the rectangles, representing tracks in the muon system, and straight (high $\mathrm{P}_{\mathrm{T}}$ ) tracks that point to the muon tracks. In Figure 9.6, the two leading jets can be seen in the calorimeter lego display along with the $\mathbb{F}_{T}$.

\subsection{Comparison to Other Measurements}

Figure 9.7 shows the result for the $t \bar{t}$ cross section from this analysis along with the Run I D $\varnothing$ and CDF dilepton measurements, the Run II CDF dilepton measurement, and the Run II DØ dilepton measurement from the Winter 2004 conferences. Table 9.5 shows a comparison of data yield, signal efficiency, and estimated background 


\begin{tabular}{|c|c|c|c|c|}
\hline Run/Event \# & Object & $\begin{array}{c}\mathrm{P}_{\mathrm{T}} \\
(\mathrm{GeV} / \mathrm{c})\end{array}$ & $\eta$ & $\begin{array}{c}\phi \\
\text { (Radians) }\end{array}$ \\
\hline $170024 / 28926073$ & $\begin{array}{l}\text { Muon } \\
\text { Muon } \\
\text { Jet } \\
\text { Jet } \\
E_{T} \\
\mathrm{M}_{\mu \mu} \\
\mathrm{H}_{T} \mu\end{array}$ & $\begin{array}{c}58.6 \\
21.1 \\
125.4 \\
67.4 \\
63.6 \mathrm{GeV} \\
58.5 \mathrm{GeV} / \mathrm{c}^{2} \\
251.4 \mathrm{GeV}\end{array}$ & $\begin{array}{c}0.48 \\
1.13 \\
0.71 \\
-0.29\end{array}$ & $\begin{array}{l}2.18 \\
0.45 \\
5.61 \\
3.79\end{array}$ \\
\hline $178423 / 35258885$ & $\begin{array}{l}\text { Muon } \\
\text { Muon } \\
\text { Jet } \\
\text { Jet } \\
\text { Jet } \\
E_{T} \\
\mathrm{M}_{\mu \mu} \\
\mathrm{H}_{T} \mu\end{array}$ & $\begin{array}{c}45.5 \\
27.9 \\
144.0 \\
43.2 \\
27.1 \\
78.6 \mathrm{GeV} \\
55.8 \mathrm{GeV} / \mathrm{c}^{2} \\
259.7 \mathrm{GeV}\end{array}$ & $\begin{array}{c}0.10 \\
0.09 \\
0.05 \\
-2.32 \\
-0.47\end{array}$ & $\begin{array}{l}0.02 \\
1.82 \\
3.77 \\
0.86 \\
2.43\end{array}$ \\
\hline 179614/4486931 & $\begin{array}{l}\text { Muon } \\
\text { Muon } \\
\text { Jet } \\
\text { Jet } \\
E_{T} \\
\mathrm{M}_{\mu \mu} \\
\mathrm{H}_{T} \mu\end{array}$ & $\begin{array}{c}78.2 \\
26.1 \\
152.3 \\
24.7 \\
55.2 \mathrm{GeV} \\
59.8 \mathrm{GeV} / \mathrm{c}^{2} \\
255.2 \mathrm{GeV}\end{array}$ & $\begin{array}{c}0.05 \\
-0.29 \\
-0.82 \\
-0.56\end{array}$ & $\begin{array}{l}3.77 \\
5.16 \\
0.94 \\
3.21\end{array}$ \\
\hline $179895 / 6545223$ & $\begin{array}{l}\text { Muon } \\
\text { Muon } \\
\text { Jet } \\
\text { Jet } \\
E_{T} \\
\mathrm{M}_{\mu \mu} \\
\mathrm{H}_{T} \mu\end{array}$ & $\begin{array}{c}37.6 \\
17.8 \\
85.0 \\
38.9 \\
92.4 \mathrm{GeV} \\
48.5 \mathrm{GeV} / \mathrm{c}^{2} \\
161.6 \mathrm{GeV}\end{array}$ & $\begin{array}{c}-0.30 \\
-0.05 \\
-0.97 \\
0.01\end{array}$ & $\begin{array}{l}5.71 \\
1.81 \\
2.69 \\
2.66\end{array}$ \\
\hline
\end{tabular}

TABLE 9.4. Kinematic variables for the muons, jets, and $\mathbb{E}_{T}$ for the four candidate events in data. 


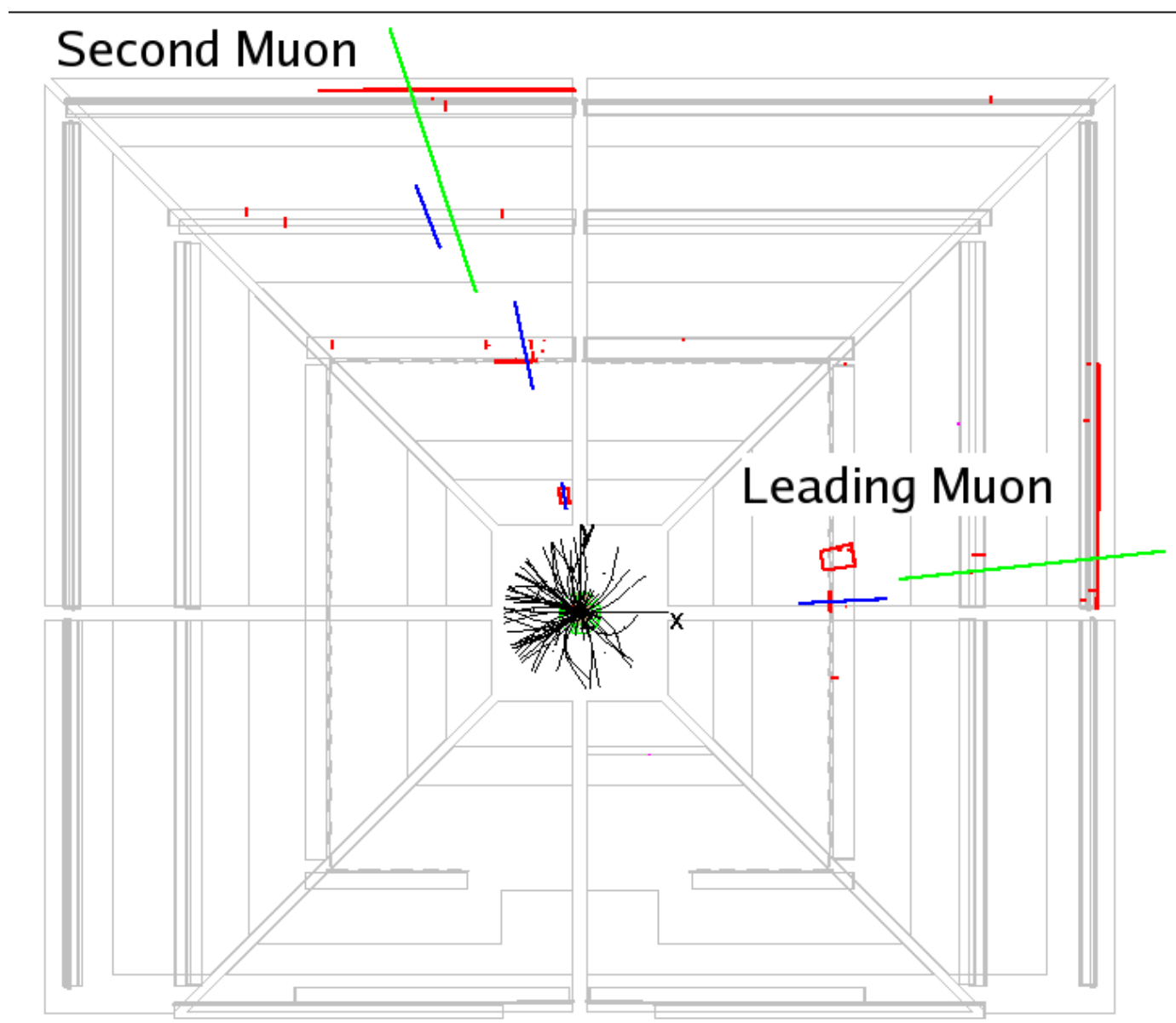

Figure 9.4. The muon display for one of the candidate events, viewed along the beam axis. 


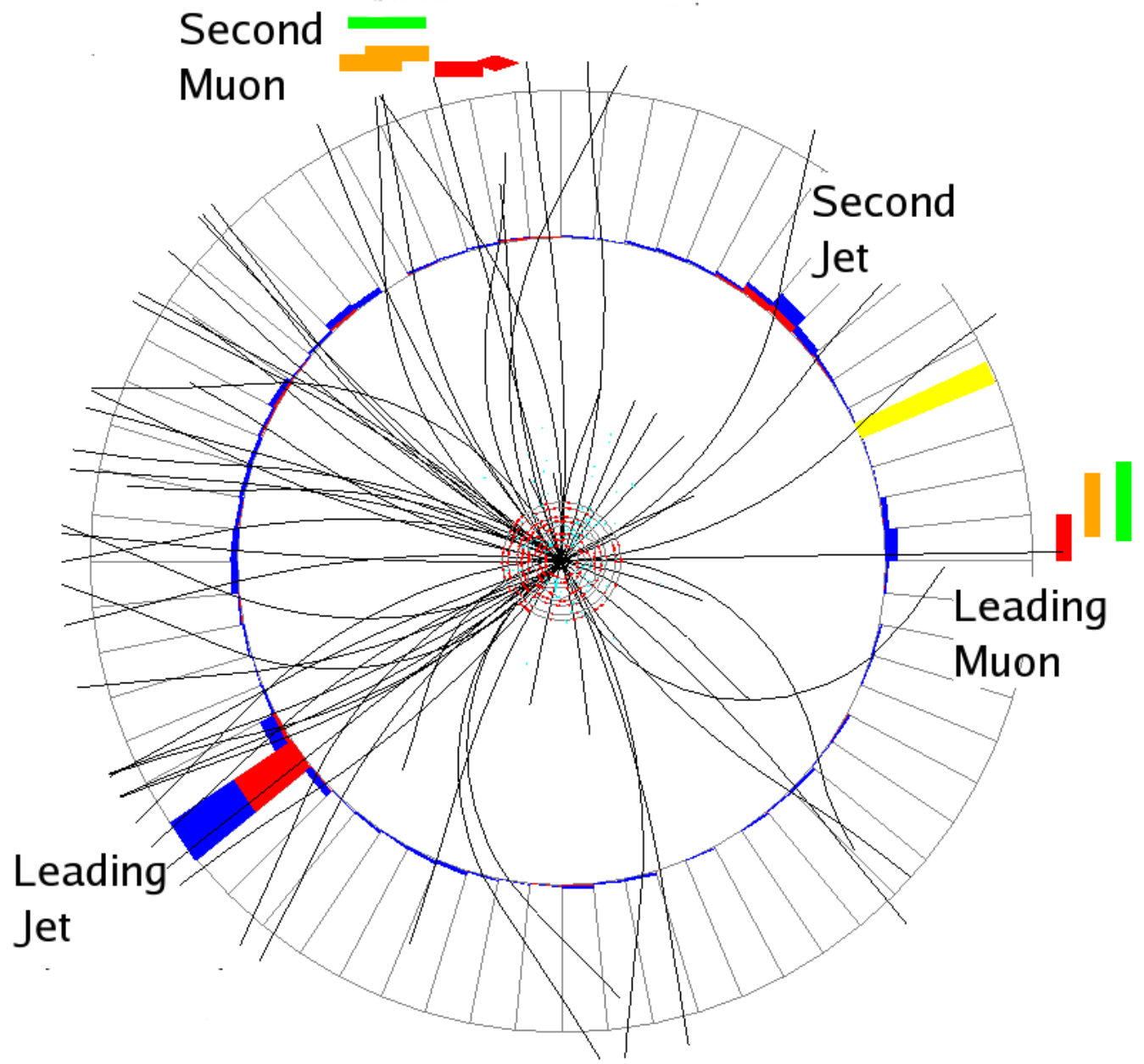

FiguRE 9.5. The central tracker and calorimeter, viewed along the beam axis. The hits in the muon system are represented by the rectangles. High $\mathrm{P}_{\mathrm{T}}$ tracks, shown as straight lines, can be seen in the central tracker extending to the muon hits. 


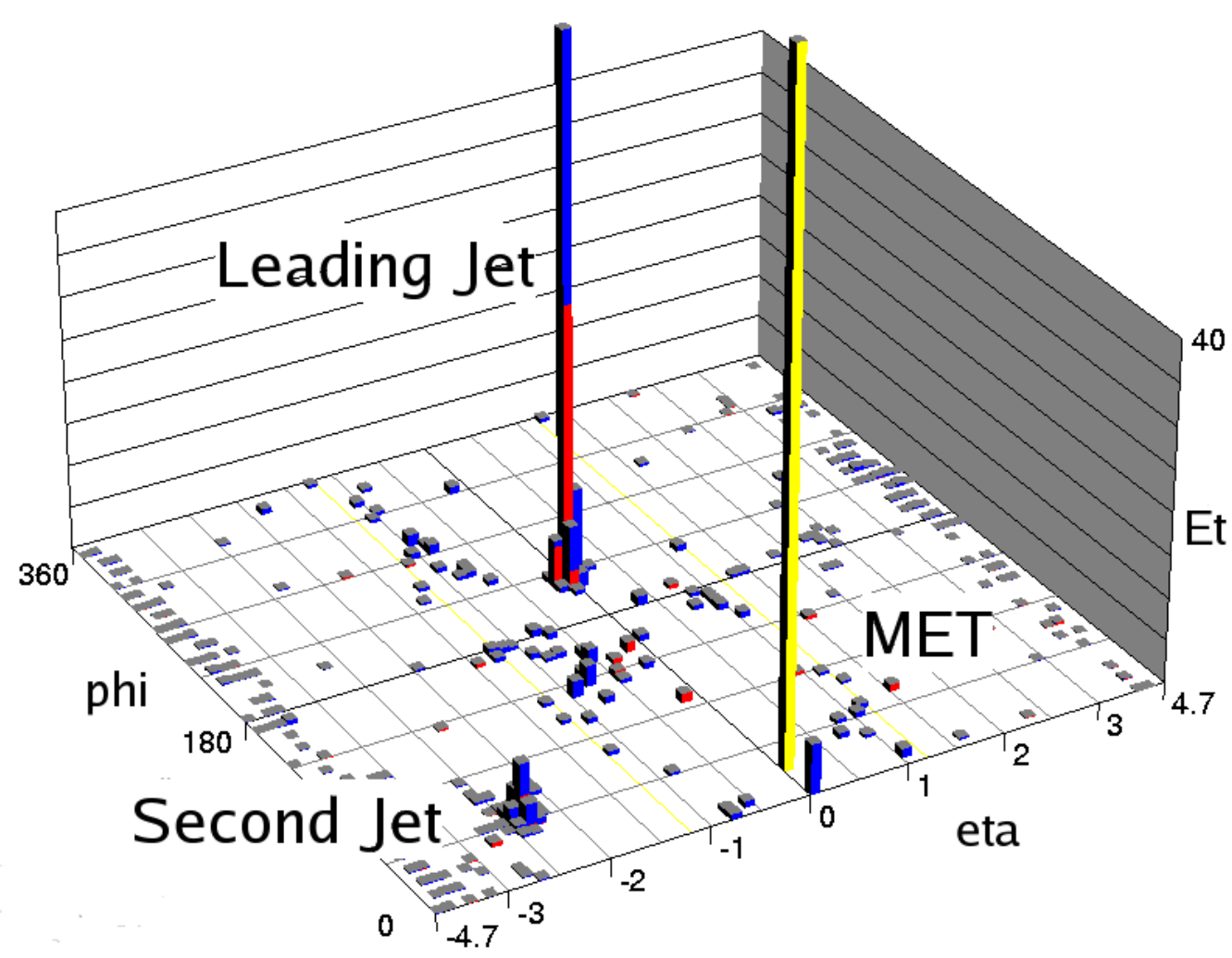

Figure 9.6. The calorimeter lego display for event $178423 / 35258885$. The leading jet has an $\mathrm{E}_{\mathrm{T}}$ of $144 \mathrm{GeV}$, while the second jet has an $\mathrm{E}_{\mathrm{T}}$ of $43 \mathrm{GeV}$. 


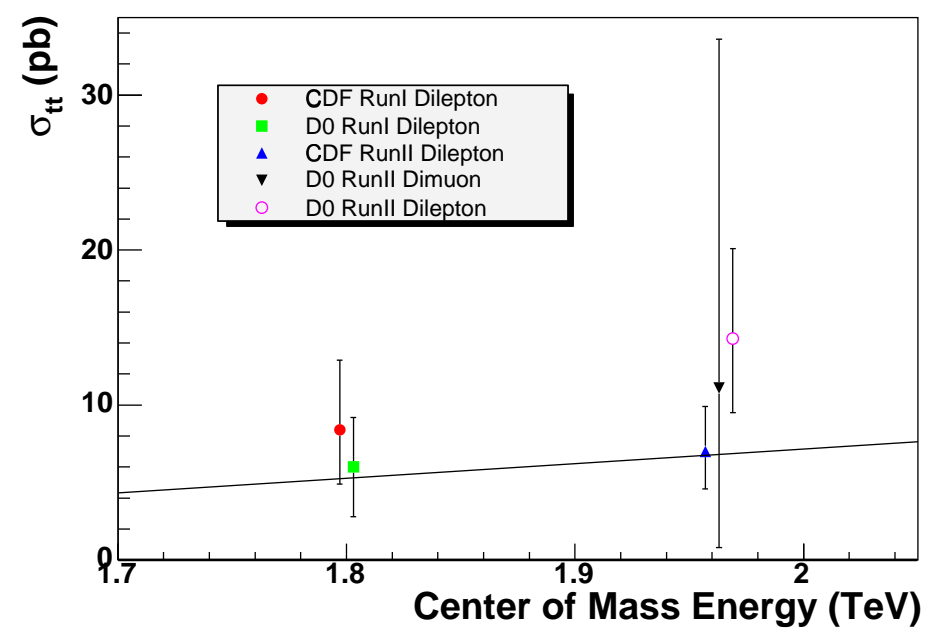

Figure 9.7. Comparison of this measurement (D $\varnothing$ Run II Dimuon) to the Run I DØ and CDF dilepton measurements and to the Run II CDF dilepton measurement. The DØ Run II dilepton result is also shown and includes this measurement and is from the Winter 2004 conference results.

yields for this analysis, that from Run I of DØ, and that from Runs I and II of CDF [7] [57] [58]. The CDF results are quoted for the dilepton (ee $+\mathrm{e} \mu+\mu \mu)$ channel, which has a factor of four increase in the branching ratio.

The signal efficiency times branching ratio for this analysis is comparable to the Run I result and about 70\% lower than the Run II CDF measurement. The largest difference in efficiency between this analysis and CDF comes from the quality cuts on the muons. The CDF analysis uses looser quality cuts on one of the muons, which increases the overall efficiency. From Table 7.1, the per muon efficiency for the isolation requirement (with the isolation data/Monte Carlo scale factor) and the distance of closest approach cut is $70 \%$. Relaxing these cuts for one muon would increase the efficiency to $8 \%$ from $5.7 \%$, a $40 \%$ increase. Another source of increased inefficiency for this analysis is the $E_{T}$ cut of $35 \mathrm{GeV}$, opposed to cuts of $20 \mathrm{GeV}$ for CDF in Run I and $25 \mathrm{GeV}$ for Run II.

The estimated background is significantly higher than the other three analysis. 


\begin{tabular}{|l|c|l|l|l|}
\hline Measurement & Events & Luminosity $(p b)$ & Background & Efficiency $x$ BR \\
\hline DØ Run II & $4 \mu \mu$ & 140 & 2.6 & $\begin{array}{l}0.09 \% \\
(\text { dimuon })\end{array}$ \\
\hline DØ Run I & $1 \mu \mu$ & 109 & 0.75 & $\begin{array}{l}0.11 \% \\
\text { (dimuon) }\end{array}$ \\
\hline CDF Run II & 13 & 200 & $\begin{array}{l}2.7 \\
(\text { dilepton total) }\end{array}$ & $\begin{array}{l}0.62 \% \\
\text { (dilepton total) }\end{array}$ \\
\hline CDF Run I & $\begin{array}{c}(3 \mu \mu) \\
(1 \mu \mu)\end{array}$ & 105 & $\begin{array}{l}2.6 \\
\text { (dilepton total) }\end{array}$ & $\begin{array}{l}0.74 \% \\
\text { (dilepton total) }\end{array}$ \\
\hline
\end{tabular}

TABLE 9.5. Summary of the number of data events, estimated background, and the signal efficiency for this measurement (DØ Run II), the Run I D $\varnothing$ result, and the Run I and Run II CDF results. Note the CDF results are for the combined dilepton results in the ee, $\mathrm{e} \mu$, and $\mu \mu$ channels, which increases the branching ratio by a factor of four.

Much of the background comes from $\mathrm{Z} / \gamma^{*}$ events that fall outside the $\mathrm{Z}$ boson mass cut due to a muon with poorly reconstructed $\mathrm{P}_{\mathrm{T}}$. Improvements for the signal efficiency and background rejection are discussed in Section 9.5.

\subsection{Future Improvements}

Several improvements can be made to this analysis, including increased signal efficiency, better background rejection, and an improved understanding of the $\mathrm{Z} / \gamma^{*}$ background simulation. The signal efficiency is relatively low (about 6\%), while the expected signal to background ratio is about $1: 3$. The uncertainty on the $\mathrm{Z} / \gamma^{*}$ background is the largest source of systematic error on the final cross section result.

The signal efficiency (Table 7.1) has several cuts that lead to significant inefficiency. The efficiency to reconstruct a medium muon is about $67 \%$. Much of this is due to lack of coverage in the central bottom of the muon detector. Some efficiency can be gained by using looser muon quality cuts on one or both of the muons, though an increase in the number of fake muons would be expected. Another potential gain in efficiency is the isolation cut (82\%), which is used to reduce the WQCD background. 
Both the muon ID and the isolation efficiencies affect both muons, so even a small increase in the per muon efficiency will lead to significant improvements in the total efficiency.

The two selection cuts used primarily for reduction of the $\mathrm{Z} / \gamma^{*}$ background, $\mathbb{E}_{T}$ and $M_{\mu \mu}$, have a combined signal efficiency of $56 \%$. Recent studies have shown that many of the $\mathrm{Z} / \gamma^{*}$ events that fall outside of the $\mathrm{Z}$ boson mass window and have large $\not_{T}$ are due to poorly reconstructed muons. Improving the muon reconstruction or rejecting events with poorly reconstructed muons would allow these cuts to be relaxed, increasing the efficiency. Alternately, the use of a Z-fitter can identify likely $\mathrm{Z}$ boson events by refitting the muon $\mathrm{P}_{\mathrm{T}}$. In this case, the $\chi^{2}$ of the returned fit is used to separate likely $\mathrm{Z}$ boson events from signal. The fit is performed with the constraints that the invariant mass of the dimuon pair equal the $\mathrm{Z}$ boson mass and the $\mathrm{P}_{\mathrm{T}}$ of the dimuon pair balance the remaining transverse energy in the event [7]. Finally, contour cuts in the $\Delta \phi\left(\mu_{\text {leading }}, \not_{T}\right)-\not_{T}$ plane have shown promise in reducing the number of poorly reconstructed $\mathrm{Z} / \gamma^{*}$ events.

Finally, reducing the uncertainty on the $\mathrm{Z} / \gamma^{*}$ background is also important. While the current Monte Carlo estimate is scaled to data with an independent data set, a method of estimating the background from data alone would provide an important cross-check. In addition to extracting the background solely from data, other cross checks could be performed such as measuring the $\mathrm{Z} / \gamma^{*}$ and $\mathrm{Z} / \gamma^{*}$ plus one jet cross sections and comparing to Monte Carlo. 


\section{Chapter 10 \\ CONCLUSiOns}

The top quark was discovered in 1995 during Run I of the Fermilab Tevatron by the DØ and CDF collaborations. Improvements in the accelerator center of mass energy and luminosity, along with upgrades to the two particle detectors for Run II, allows precision measurements of several top quark properties. These include improved mass and cross section results and measurements of other top quark properties, including the helicity of $\mathrm{W}$ bosons from top quark decay and spin correlations in the $t \bar{t}$ system. An improved measurement of the $t \bar{t}$ production cross section is an important test of Standard Model predictions for heavy quark production. In addition, it can place limits on theories beyond the Standard Model, such as topcolor, that predict increased production of $t \bar{t}$ pairs.

The measurement of the $t \bar{t}$ production cross section proceeds by isolating likely signal events using a series of selection criteria, or cuts, on the data. These cuts are designed to retain as many signal events as possible while rejecting background events. In this analysis, events where both $\mathrm{W}$ bosons from the decay $t \rightarrow W b$ decayed to muons were used. The signature in the detector was two high $\mathrm{P}_{\mathrm{T}}$ muons from the decay of the $\mathrm{W}$ bosons, two high $\mathrm{E}_{\mathrm{T}}$ jets from the b-quarks, and missing transverse energy $\left(\not_{T}\right)$ from the neutrinos produced in the decay of the $\mathrm{W}$ bosons.

To measure the cross section, four values must be measured. The first is the number of data events, which is simply the number of events that pass all of the selection criteria. The second is the total efficiency for signal events to be recorded in the data and pass all of the selection cuts. This was studied using data and signal Monte Carlo. When possible, values derived from Monte Carlo were checked with data and data/Monte Carlo scale factors were applied. The third is the expected 
number of background events in the sample. This was estimated from data and Monte Carlo for this analysis. Finally, the total luminosity for the data set must be determined. This is done by the DØ luminosity system, described in Chapter 6 .

The efficiency for signal events to pass all cuts was measured primarily with signal Monte Carlo (Chapter 7) and found to be 5.7\%. Four scale factors were applied to correct for differences between the data and Monte Carlo for muon identification, muon isolation, muon vertex cuts, and central track finding efficiencies (Table 7.1). The cuts that have the lowest efficiency include the requirement that the event have two well-reconstructed muons, that both muons be isolated, and that the reconstructed invariant mass of the muons fall outside of a $40 \mathrm{GeV} / \mathrm{c}^{2}$ window around the $\mathrm{Z}$ boson mass.

The dominant background is $\mathrm{Z} / \gamma^{*} \rightarrow \mu \mu$, which accounts for almost $80 \%$ of the expected background. Two cuts were used to reduce this background. The first was to require that the dimuon invariant mass was outside a $40 \mathrm{GeV} / \mathrm{c}^{2}$ window around the $\mathrm{Z}$ boson mass $\left(\mathrm{M}_{\mu \mu}<70 \mathrm{GeV} / \mathrm{c}^{2}\right.$ or $\left.\mathrm{M}_{\mu \mu}>110 \mathrm{GeV} / \mathrm{c}^{2}\right)$, since most of the events inside this window are real $\mathrm{Z}$ bosons with a mass of $90 \mathrm{GeV} / \mathrm{c}^{2}$. Secondly, since the $\mathrm{Z} / \gamma^{*}$ events have no real $E_{T}$ (because there are no neutrinos), the total $E_{T}$ was required to be above $35 \mathrm{GeV}$. The $\mathrm{Z} / \gamma^{*}$ background estimate came from Monte Carlo that was normalized to a $\mathrm{Z}$ control yield in data. Many of the $\mathrm{Z} / \gamma^{*}$ events that pass these two cuts in Monte Carlo were found to have muons with poorly reconstructed transverse momentum. This allows the invariant mass to fall outside of the $\mathrm{Z}$ boson mass window and can lead to fake $E_{T}$, since the $E_{T}$ is corrected for muons in the event. The other backgrounds considered were WQCD events, were one or both of the muons fake isolation from nearby jets, and WW +2 jet events, which have a similar signature to the signal. A full description of the background estimation is given in Chapter 8.

After all selection cuts are applied to the data, a total of four events remain, with an expected background of 2.61 events. The final result for the cross section 
was found using the Feldman-Cousins approach for counting experiments with low statistics. The systematic error on $\sigma_{t \bar{t}}$ is dominated by uncertainties on the estimated $\mathrm{Z} / \gamma^{*}$ background (Table 9.2). The measured value for the $t \bar{t}$ production cross section is

$$
\sigma_{t \bar{t}}=11.1_{-9.3}^{+22.1} \text { (stat.) }{ }_{-4.5}^{+4.3} \text { (sys.) } p b
$$

and is consistent with the NNLO theoretical prediction of $6.77 \mathrm{pb}$ for a top quark mass of $175 \mathrm{GeV} / \mathrm{c}^{2}$.

There are several possible improvements to the analysis. The muon quality cuts are the largest source of inefficiency and relaxed or improved cuts on one or both of the muons will lead to a large increase in efficiency. The dominant $\mathrm{Z} / \gamma^{*}$ background can be reduced by improving the muon $\mathrm{P}_{\mathrm{T}}$ resolution or the use of a Z-fitter. Finally, estimates of the $\mathrm{Z} / \gamma^{*}$ background from data and improved cross-checks between data and Monte Carlo will reduce the uncertainty on the estimated background. 


\section{Appendix A \\ Level 1 Muon Trigger Inputs}

\section{A.1 MTC05}

The MTC05 cards in the central and forward regions match tracks from the L1CTT trigger to scintillator hits in the muon system. The tracks from the L1CTT carry $\mathrm{P}_{T}$, $\phi$, and sign information.

\section{A.1.1 L1CTT Inputs}

The tracks in the L1CTT are formed in $4.5^{\circ}$ trigger sectors, numbered 0-79. Trigger sector 0 spans $0-4.5^{\circ}\left(0^{\circ}\right.$ is $+\mathrm{x}$ in the $\mathrm{D} \varnothing$ coordinate system.) Thus, octant 0 in the muon system corresponds to sectors 0-9, octant 1 to sectors 10-19, etc.

The L1CTT inputs to the MTC05's come from the DFEA boards located in two crates on the central platform (PC03-01, PC03-02). Each DFEA crate has 20 DFE motherboards, and each motherboard holds two daughter cards. The daughtercards form triggers for one $4.5^{\circ}$ sector of the CTT. The outputs from one DFE (two sectors) are sent to a transition module that plugs into the back of the backplane and holds two serial transmitters, which send the tracks to L1MU.

Each sector can transmit up to six tracks to L1MU. Each track is encoded as a 16-bit word (Table A.1) and the tracks are ordered in $\mathrm{P}_{T}$. If more than six tracks are found in a given sector, the six with the highest $\mathrm{P}_{T}$ are sent.

In addition to tracks from the 80 sectors, the sectors that lie on octant boundaries (the so-called overlap sectors) send tracks to two sectors - the 'home' octant and the adjacent octant. For example, sector 9 corresponds to the last sector in octant 0 . The tracks from this sector are sent to the MTC05 for octant 0 and octant 1 . The 


\begin{tabular}{|l|c|}
\hline Bits & Description \\
\hline$[5 . .0]$ & H-layer $\phi$ position \\
\hline$[8 . .6]$ & Extended $\mathrm{P}_{T}$ \\
\hline$[10 . .9]$ & $\mathrm{P}_{T}$ \\
\hline$[11]$ & Curvature \\
\hline$[14 . .12]$ & No Connect \\
\hline$[15]$ & Valid Track \\
\hline
\end{tabular}

TABLE A.1. CTT data formats to L1MU. The six highest $\mathrm{P}_{T}$ tracks from each sector are sent, along with a parity word for error checking. Each 16-bit word gives the $\phi$ position in the outer $(\mathrm{H})$ layer of the CTT, information about $\mathrm{P}_{T}$, and the curvature in the magnetic field.

transmitters for these sectors have two outputs.

A total of 96 cables come from the L1CTT. All L1CTT inputs are used by the central, north, and south regions. The inputs from the DFEA's are sent to a splitter crate (PE04-0, A.5) where the signals are split and sent to the three regions.

\section{A.1.2 Central Scintillators}

The central scintillator inputs come from six scintillator front end crates located in the central muon trusses. Each FE crate holds several scintillator front end cards (SFE's), each of which can digitize up to 48 photomultiplier inputs. Each SFE has a serial transmitter to send hits to L1MU. The scintillator crate to octant mapping is given in table A.2, with a detailed mapping in ref ??.

\begin{tabular}{|l|l|l|}
\hline Crate & Octants & SFE's \\
\hline x50 & 0,7 & 4 CMSC, 1 B- $\phi, 4$ A- $\phi$ \\
\hline x51 & 1 & 2 CMSC, 2 A- $\phi$ \\
\hline x52 & 2 & 2 CMSC, 2 A- $\phi$ \\
\hline x53 & 3,4 & 4 CMSC, 1 B- $\phi, 4$ A- $\phi$ \\
\hline x55 & 5 & 2 CMSC, 2 B- $\phi, 1$ A- $\phi$ \\
\hline x56 & 6 & 2 CMSC, 2 B- $\phi, 1$ A- $\phi$ \\
\hline
\end{tabular}

TABLE A.2. Central scintillator front end crates. 
To allow all scintillator inputs to be sent to the MTC05, some scintillator inputs are passed through a concentrator card before being sent to L1MU. Since each SFE handles a maximum of 48 inputs, the serial outputs only use half (48/96) of the available bits/BC. The concentrator cards combine the outputs of two SFE's onto one serial link and send the data to L1MU. In the central region, all C-layer inputs are concentrated, along with the A- $\phi$ inputs for octants 4 and 7 .

The format of data from the SFE's is shown in Table A.3. The regular output of the SFE's comes on strobes 1,2, and 3. When two inputs are combined in a concentrator, the 48 bits from the first SFE are on strobes 0-2, while the data for the second SFE are on strobes 3-5. Parity is always on strobe 6.

\begin{tabular}{|c|c|l|}
\hline Strobe & Normal & Concentrated \\
\hline 0 & $\mathrm{x}$ & $\mathrm{SFE} 0[15 . .0]$ \\
\hline 1 & SFE0 $[15 . .0]$ & $\mathrm{SFE} 0[31 . .16]$ \\
\hline 2 & SFE0 $[31 . .16]$ & $\mathrm{SFE} 0[47 . .32]$ \\
\hline 3 & SFE0 $[47 . .32]$ & $\mathrm{SFE} 1[15 . .0]$ \\
\hline 4 & $\mathrm{x}$ & $\mathrm{SFE} 1[31 . .16]$ \\
\hline 5 & $\mathrm{x}$ & SFE1 $[47 . .32]$ \\
\hline 6 & Parity & Parity \\
\hline
\end{tabular}

TABLE A.3. Scintillator data formats to L1MU.

\section{A.1.3 Forward Scintillators}

The forward scintillator inputs come from six front end crates in each the north and south regions. Every octant for each layer requires two SFE's. The AB crates are located in the EF trusses and all contain four A-pixel and four B-pixel SFE's. The two C-layer crates are in the EC trusses and contain eight C-pixel SFE's each. Table A.4 shows the crate configuration for the north. In the forward region, all B- and C-layer inputs are concentrated to allow twelve CTT inputs for each MTC05. 


\begin{tabular}{|l|l|l|}
\hline Crate & Octants & SFE's \\
\hline x70 & 0,1 & 4 Apix, 4 Bpix \\
\hline x72 & 2,3 & 4 Apix, 4 Bpix \\
\hline x74 & 4,5 & 4 Apix, 4 Bpix \\
\hline x76 & 6,7 & 4 Apix, 4 Bpix \\
\hline x278 & $0,1,6,7$ & 8 Cpix \\
\hline x279 & $2,3,4,5$ & 8 Cpix \\
\hline
\end{tabular}

TABLE A.4. North scintillator front end crates. The south has the same configuration with crate numbers x90, x92, etc.

\section{A.1.4 MTC05 Inputs}

Figures A.1 and A.2 show the CTT and scintillator inputs to the central and forward MTC05 cards. The CTT inputs are on the low 12 receivers while the scintillator inputs are on the upper four. The differences in the scintillator inputs in central octants 4-7 are due to B-layer counters that have been added to increase coverage around the calorimeter supports.

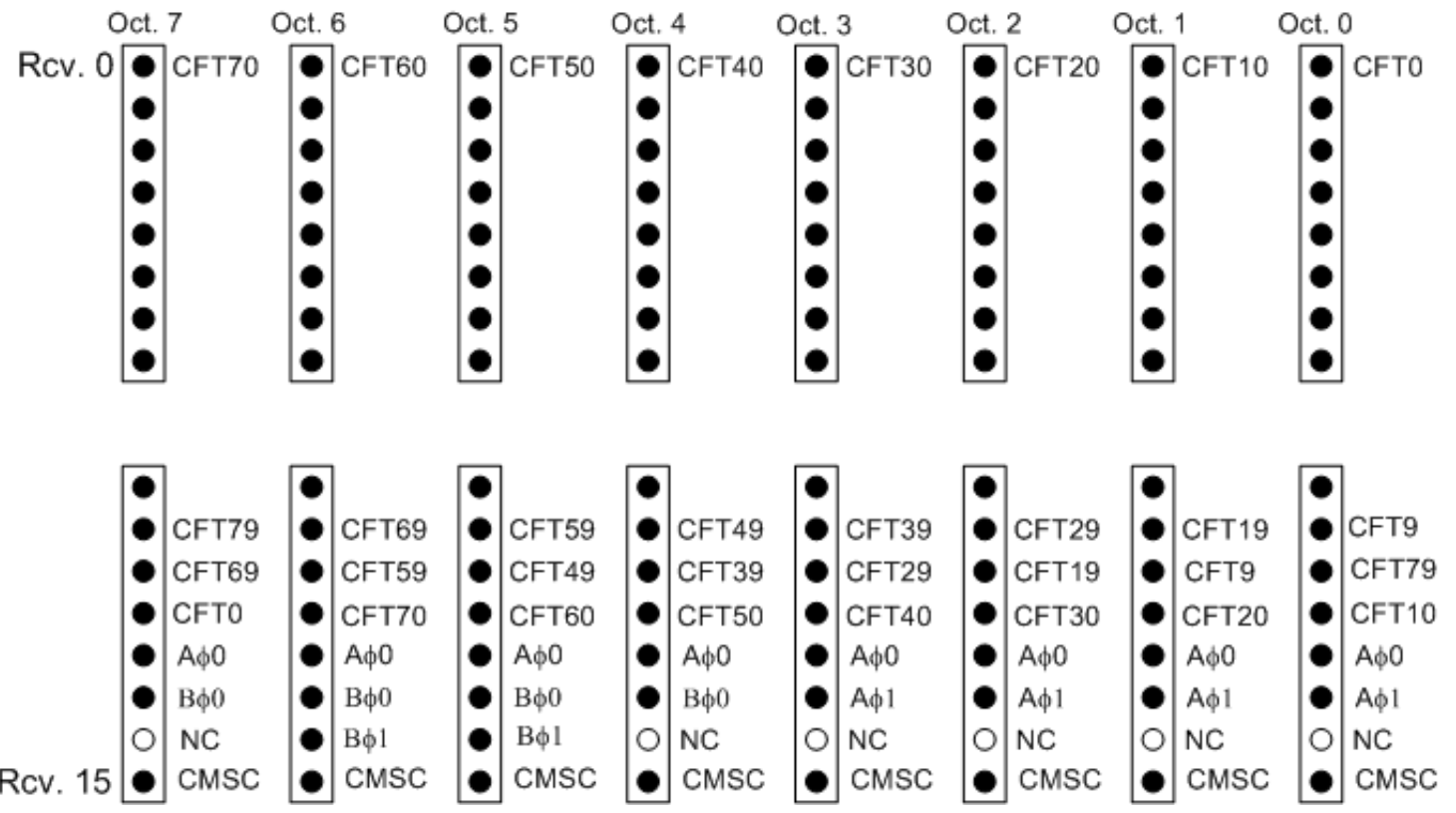

Figure A.1. MTCC 05 inputs. 


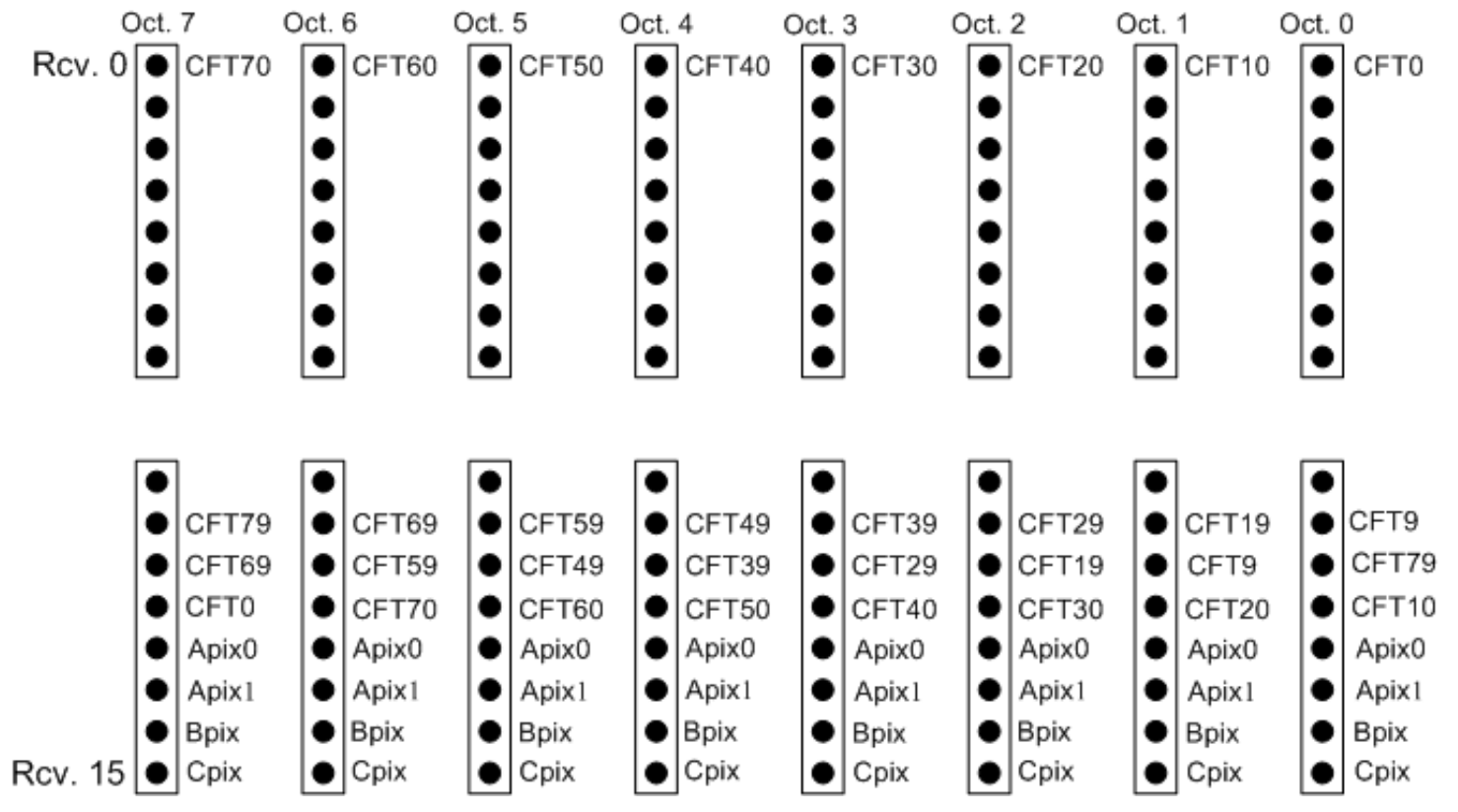

Figure A.2. MTCN 05 inputs. 


\section{A.2 MTC10}

The MTC10 cards form triggers based on hits in the wire chambers. Track stubs, or centroids, are first formed in the three layers. These are used to confirm scintillator hits in the same layer. Finally, coincidences between confirmed scintillator hits in different layers are used to form triggers.

\section{A.2.1 Central}

In the central region, the wire chambers are the Proportional Drift Tube (PDT) chambers. A typical octant has thirteen chambers (three A-, five B-, and 5 C-layer). There are a total of 94 PDT's, and each has front-end electronics (the Front End Boards and Control Boards) mounted directly on the chambers. Each control board has one serial transmitter that sends trigger hits to L1MU. Because the $550 \mathrm{~ns}$ drift time in the PDT's is longer than the 132 ns bunch spacing, the trigger hits are held high for multiple crossing and a scintillator hit is used to 'tag' the correct bunch crossing for the hit.

\begin{tabular}{|c|l|}
\hline Strobe & Data \\
\hline 0 & Wire[15..0] \\
\hline 1 & Wire[31..16] \\
\hline 2 & Wire[47..32] \\
\hline 3 & Wire[63..48] \\
\hline 4 & Wire[79..64] \\
\hline 5 & Wire[95..80] \\
\hline 6 & Parity \\
\hline
\end{tabular}

TABLE A.5. PDT data format for wire hits to L1MU.

The A-layer chambers on the sides of the detector span two octants. This means that octants $0 \& 7$ share three A-layer chambers, as do octants $3 \& 4$. The inputs from these chambers are sent through a splitter and then sent to both octants. By using the A- $\phi$ counters to confirm the PDT hits, the octant ambiguity is removed. 
All central scintillator hits go through splitter cards and are sent to both the central MTC05 and MTC10 cards. The scintillator formats are identical to the central MTC05 inputs (table A.3).

\section{A.2.2 Forward}

In the forward region, the large number of MDT channels (roughly 50,000) makes it impossible to send the MDT hits directly to the MTC10's. Instead, the raw hits are first sent to Muon Centroid Cards (MCEN's) where centroids are formed in each layer and then sent to the MTC10 cards.

The MDT digitizing cards (MDC's) reside in crates in the EF and EC trusses. Each MDC digitizes up to 192 MDT channels and has two serial transmitters for transfer to the MCEN cards. Each crate handles two octants for a given layer (Table A.6). The A- and B-layer crates are located at the corners of the End Iron toroids, while the C-layer crates are in the EC trusses.

\begin{tabular}{|l|l|l|}
\hline Crate & Layer & Octants \\
\hline x60 & $\mathrm{A}$ & 0,1 \\
\hline x62 & $\mathrm{A}$ & 2,3 \\
\hline x64 & $\mathrm{A}$ & 4,5 \\
\hline x66 & $\mathrm{A}$ & 6,7 \\
\hline x160 & $\mathrm{B}$ & 0,1 \\
\hline x162 & $\mathrm{B}$ & 2,3 \\
\hline x164 & $\mathrm{B}$ & 4,5 \\
\hline x166 & $\mathrm{B}$ & 6,7 \\
\hline x260 & $\mathrm{C}$ & 0,1 \\
\hline x262 & $\mathrm{C}$ & 2,3 \\
\hline x264 & $\mathrm{C}$ & 4,5 \\
\hline x266 & $\mathrm{C}$ & 6,7 \\
\hline
\end{tabular}

TABLE A.6. North MDT front end crates. The south has the same configuration with crate numbers $\mathrm{x} 80$, x82, etc.

The MCEN's receive the data from the MDC cards, decode the inputs, and form 
centroids. The data sent to the MTC10 cards is the centroid index, which is the projection of the centroid to the innermost deck of the chamber, with centroid index 0 is located closest to the beam pipe.

At the forward MTC10, the centroids found by the MCEN's are used to confirm forward scintillator hits. Coincidences between confirmed scintillator hits in different layers then form triggers. As in the central, all forward scintillator hits are sent to splitter cards so they can be used by the MTC05 and MTC10 cards.

\section{A.2.3 MTC10 Inputs}

Figures A.3 and A.4 show the input mapping for the central and forward MTC10's. In the central, there are 13 PDT's for every octant except the bottom two (octants 5 \& 6), which have 11. The scintillator inputs come in on the remaining channels.

In the forward regions, there are three A-layer, four B-layer, and four C-layer centroid inputs for all octants. The scintillator inputs use four of the remaining channels.
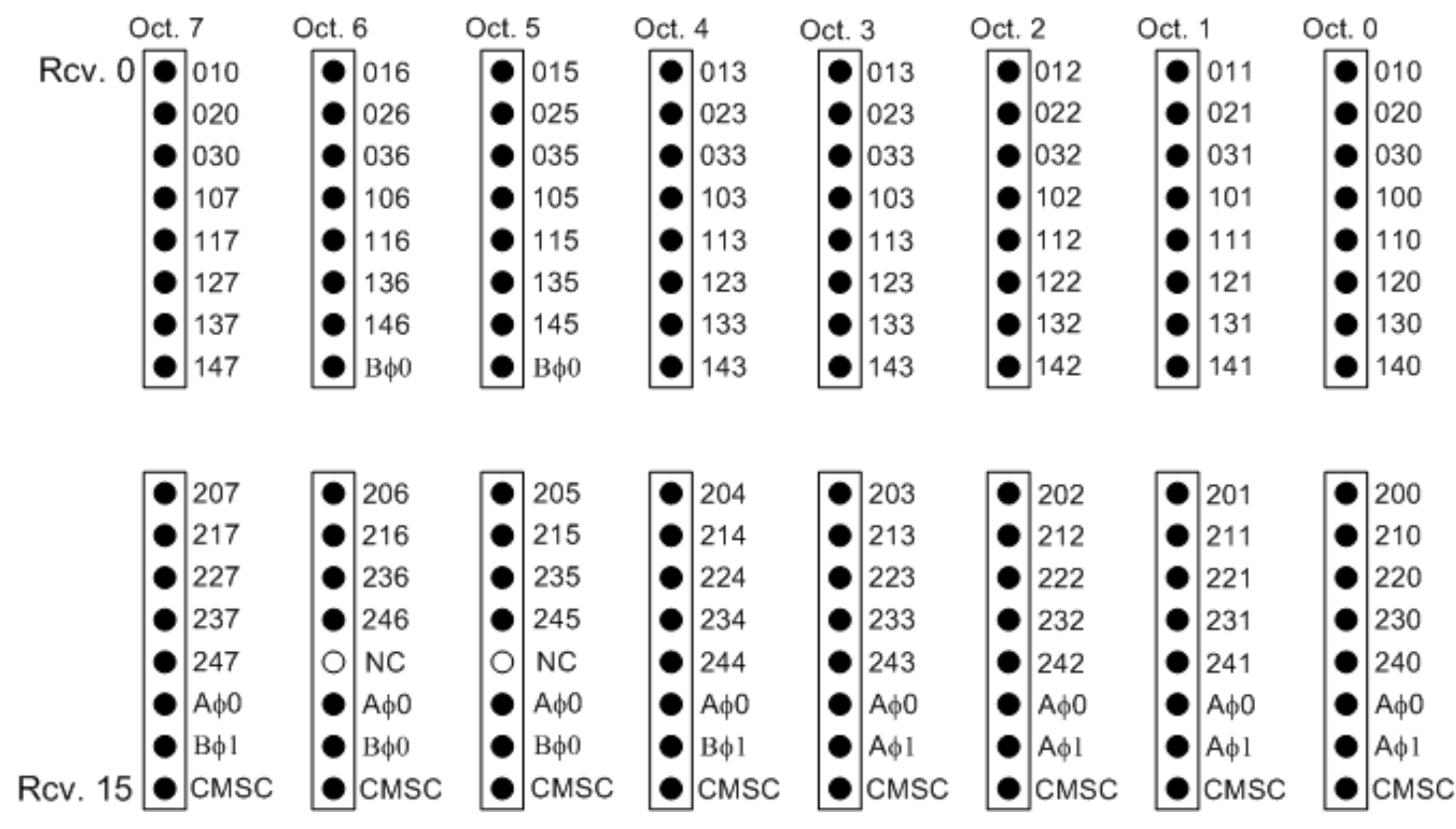

Figure A.3. Central MTC10 inputs. 

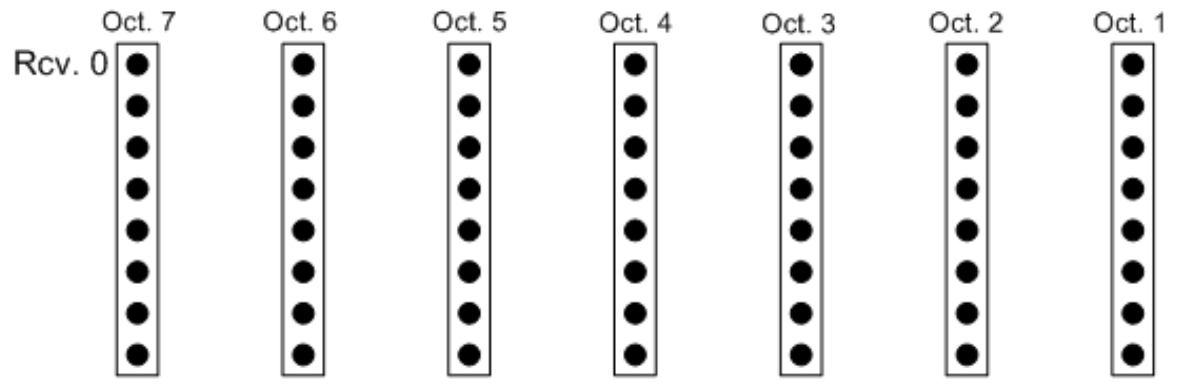

Oct. 0

- A-MDT 0

- A-MDT 1

A-MDT 2

B-MDT 0

B-MDT 1

B-MDT 2

B-MDT 3

Rcv. 15
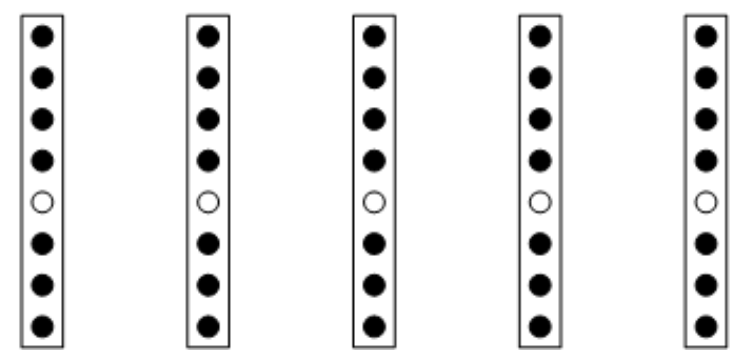

\begin{tabular}{|l|}
\hline \\
0 \\
0 \\
0 \\
0 \\
0 \\
0
\end{tabular}

\begin{tabular}{|l|l}
\hline & C-MDT 0 \\
C-MDT 1 \\
C-MDT 2 \\
C-MDT 3 \\
C.M \\
Apix 0 \\
Apix 1 \\
Cpix
\end{tabular}

Figure A.4. Forward MTC10 inputs. 


\section{A.3 Splitters}

The splitter crates are used when an input from a given front end must be sent to more than one trigger card. The splitter cards receive one serial input and send out three copies. In cases where only two outputs are required, the third must be terminated. Each splitter card has eight channels.

The L1CTT inputs must be sent to the central, north, and south trigger crates. Figure A.5 shows the mapping at the front of this splitter crate. Channel 0 of every card handles inputs for the octant 0 trigger cards, channel 1 handles octant 1 , etc. The overlap inputs for a given octant are on the last two splitter cards in the crate (labeled sect. -1 and sect. +1 ).

All scintillator inputs are sent through splitters so they can be sent to both the MTC05 and MTC10 trigger cards. Figure A.6 shows the mapping for the central/north splitter. This splitter crate handles the central A- and B-layer scintillators, the north scintillators, and the A-layer PDT's for octants $0 \& 7$ and $3 \& 4$. Figure A.7 shows the mapping for the central C-layer and south scintillator inputs.

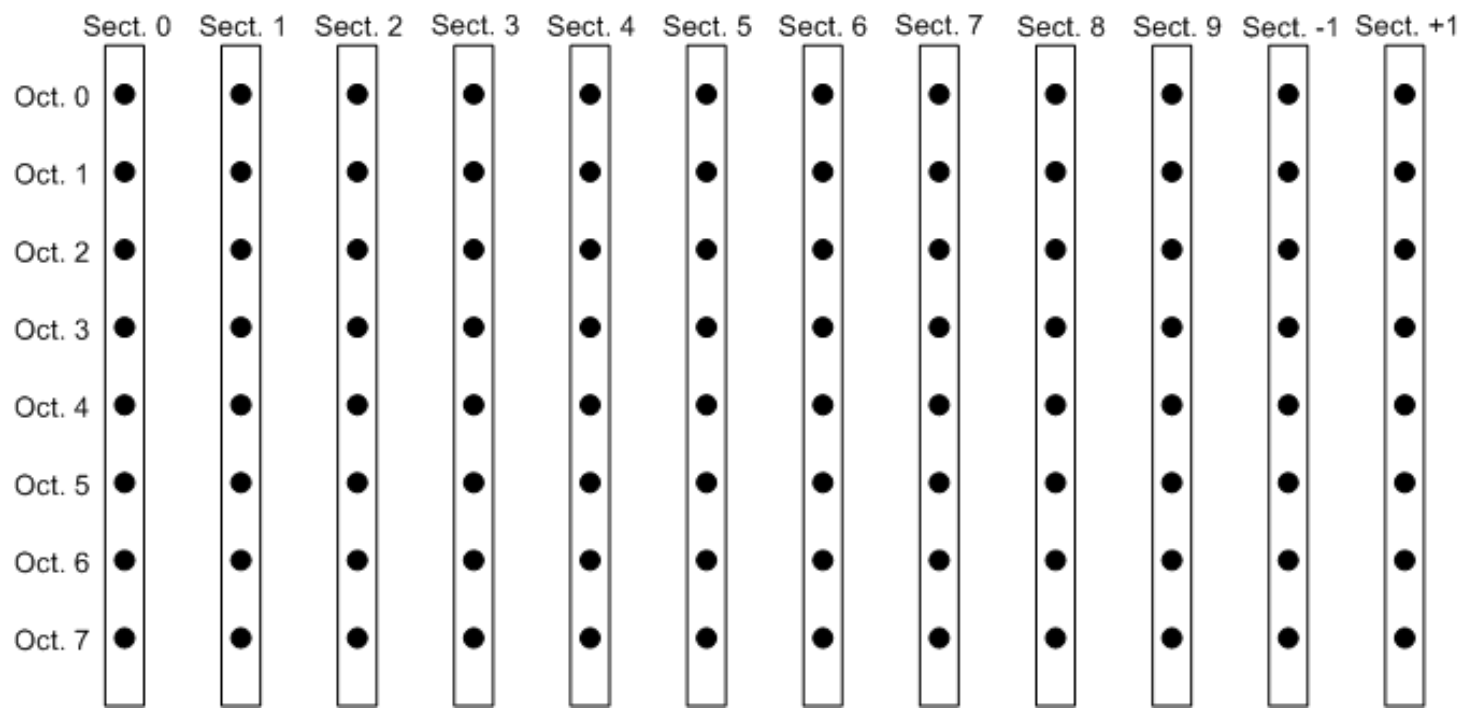

Figure A.5. CTT Splitters. 


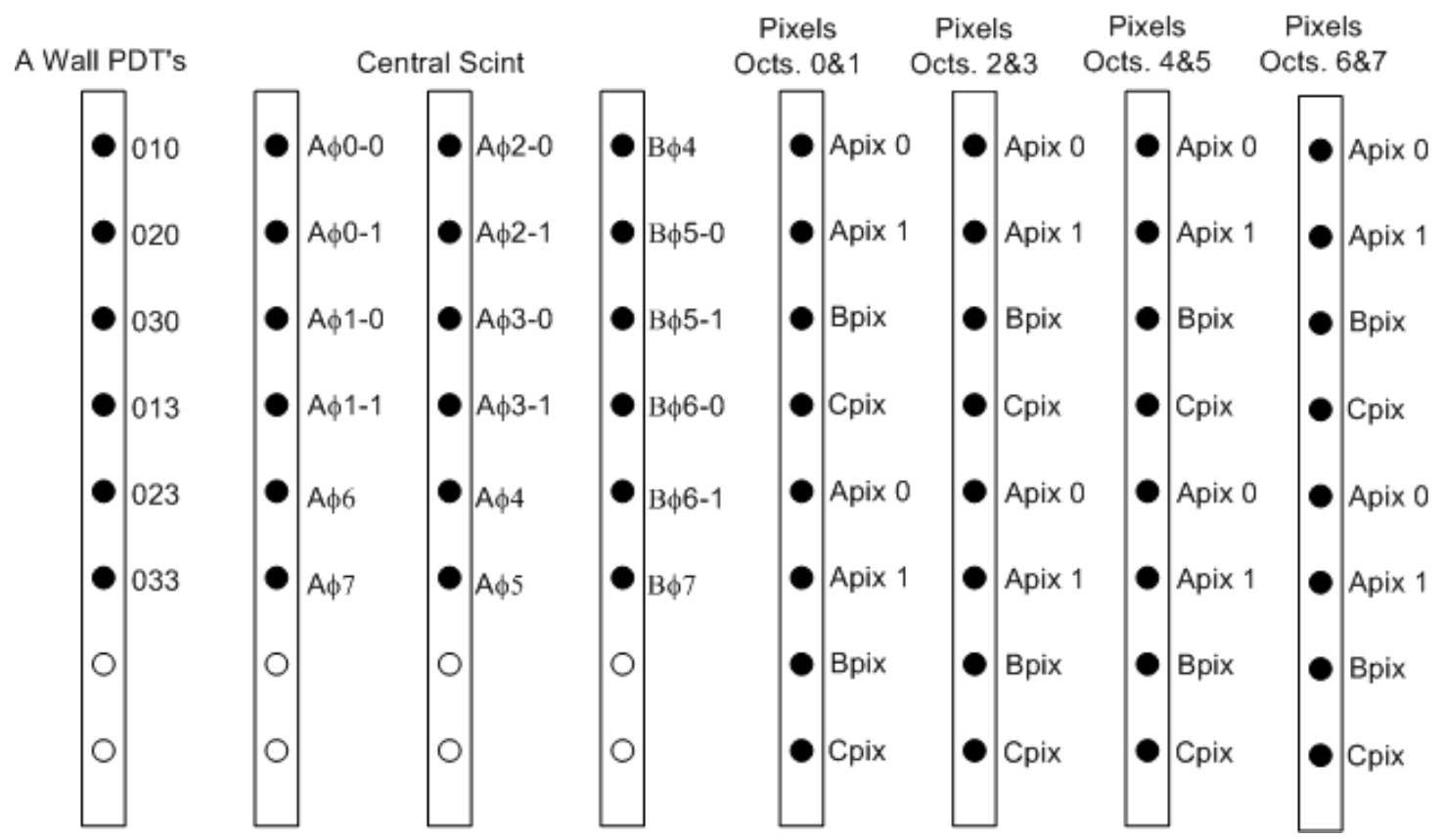

Figure A.6. Central/North Splitters.

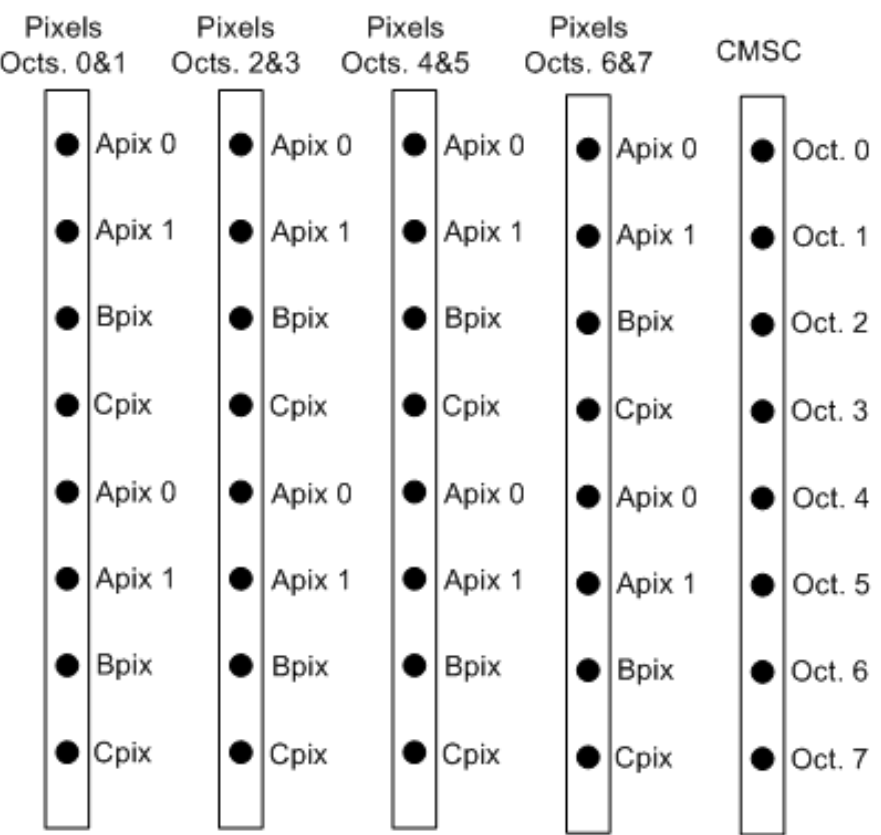

Figure A.7. South Splitters. 


\section{REFERENCES}

[1] S. F. Novaes, "Standard Model, an Introduction," [hep-ph/0001283] (2000).

[2] G. Altarelli, "The Electroweak Interactions in the Standard Model and Beyond," [hep-ph/0406270] (2004).

[3] K. Lane, "Two Lectures on Technicolor," [hep-ph/0202255] (2002).

[4] S. Abachi et al. [D0 Collaboration], Phys. Rev. Lett. 74, 2632 (1995) [hepex/9503003].

[5] F. Abe et al. [CDF Collaboration], Phys. Rev. Lett. 74, 2626 (1995) [hepex/9503002].

[6] The CDF Collaboration, the DØ Collaboration, and the TEVATRON Electroweak Working Group, "Combination of CDF and DØ Results on the Top Quark Mass," [hep-ph/0404010] (2004).

[7] V. M. Abazov et al. [D0 Collaboration], Phys. Rev. D 67, 012004 (2003) [hepex/0205019].

[8] T. Affolder et al. [CDF Collaboration], Phys. Rev. D 64, 032002 (2001) [Erratumibid. D 67, 119901 (2003)] [hep-ex/0101036].

[9] R. M. Harris, C. Hill, and S. J. Parke, " Cross Section for Topcolor Z ${ }_{t}^{\prime}$ Decaying to $t \bar{t}, "$ [hep-ph/9911288] (1999).

[10] E. Simmons, "Top Physics," [hep-ph/0011224] (2000).

[11] N. Kidonakis and R. Vogt, Phys. Rev. D 68, 114014 (2003) [hep-ph/0308222].

[12] M. Gluck, J. F. Owens and E. Reya, Phys. Rev. D 17, 2324 (1978).

[13] S. Eidelman et al. [Particle Data Group Collaboration], Phys. Lett. B 592, 1 (2004).

[14] J. Pumplin, D. R. Stump, J. Huston, H. L. Lai, P. Nadolsky and W. K. Tung, JHEP 0207, 012 (2002) [hep-ph/0201195].

[15] E. Laenen, J. Smith and W. L. van Neerven, Phys. Lett. B 321, 254 (1994) [hep-ph/9310233].

[16] N. Kidonakis, E. Laenen, S. Moch and R. Vogt, Phys. Rev. D 64, 114001 (2001) [hep-ph/0105041]. 
[17] M. Jezabek and J. H. Kuhn, Nucl. Phys. B 314, 1 (1989).

[18] J. Thompson, "Introduction to colliding Beams at Fermilab," DØ Note 2367 (1994).

[19] Fermilab Beams Division, "Main Injector Rookie Book," http://www-bdnew.fnal.gov/operations/rookie_books/rbooks.html (2003).

[20] S. Abachi et al. [D0 Collaboration], Nucl. Instrum. Meth. A 338, 185 (1994).

[21] S. Abachi et al. [D0 Collaboration], FERMILAB-PUB-96-357-E (1996).

[22] E. Kajfasz, "DØ Silicon Microstrip Tracker for Run IIa," [hep-ex/0112014] (2001).

[23] T. Zimmerman it et al., "The SVXII Readout Chip," IEEE Trans. Nucl. Sci. NS42 (1995) 803.

[24] DØ Collaboration, "The DØ Upgrade Central Fiber Tracker," http://d0server1.fnal.gov/users/stefan/www/CFT_TDR/CFT_TDR.ps (1997).

[25] M. Adams, et al., "Design Report of the Central Preshower Detector for the DØUpgrade," DØ Note 3014 (1996).

[26] A. Gordeev, "Technical Design Report of the Central Preshower Detector for the DØUpgrade," DØ Note 3445 (1998).

[27] L. Groer, "DØ Calorimeter Upgrades for Tevatron Run II," DØ Note 4240 (2003).

[28] B. Baldin et al., "Technical Design of the Central Muon System," DØ Note 3365 (1997).

[29] G. Alexeev et al., "Technical Design Report for the DØ Forward Muon Tracking Detector Based on Mini-drift Tubes," DØ Note 3366 (1997).

[30] V. Abramov et al., "Technical Design Report for the DØ Forward Muon Trigger Scintillation Counters," DØ Note 3237 (1997).

[31] D. Denisov, "The DØ Detector Muon System," DØ Note 4440 (2004).

[32] J. Linneman, "The DØ Level 2 Trigger," DØ Note 3334 (1997).

[33] D. Chapin et al., eConf C0303241, TUGP010 (2003) [eConf C0303241, MOGT002 (2003)] [physics/0307070]. 
[34] J. Steinberg, "I/O Specification for Serial Transmitter Daughter Board (PCB0140-XMIT)," http://atlas.physics.arizona.edu/ johns/11muo/11mu/ Transmitter_Specs_07_07_99.pdf (1999).

[35] J. Steinberg, "I/O Specification for Serial Receiver Daughter Board (PCB-0140XMIT)," http://atlas.physics.arizona.edu/ johns/11muo/11mu/

Receiver_specs_01_18_00.pdf (2000).

[36] K. Johns, J. Steinberg, "Muon Trigger Card, Functional Description," http://atlas.physics.arizona.edu/ johns/11muo/11mu/ mtcm_specs_07-15-02_Rev_C.pdf

[37] K. Johns, J. Steinberg, "Muon Trigger Crate Manager, Functional Description," http://atlas.physics.arizona.edu/ johns/11muo/11mu/ mtcm_specs_07-15-02_Rev_C.pdf (2002).

[38] J. Temple, R. McCroskey, and K. Johns, "Upgrade of Level 1 Muon Trigger Logic for the v13 Trigger List," DØ Note 4566 (2004).

[39] K. Johns, "Level 1 Muon Trigger Terms Description," http://atlas.physics.arizona.edu/ johns/11muo/11musim/ 11muterms_description.pdf

[40] C. Clément et al., "MuonID Certification for p14," DØ Note 4350 (2004).

[41] Common Sample Group, http://www-d0.fnal.gov/Run2Physics/cs/index.html.

[42] M. Klute, L. Phaf, and D. Whiteson, "TopAnalyze - A Framework Analyze Package For Top Group Analyses," DØ Note 4122 (2004).

[43] The Top Physics Group of the DØ Collaboration, "DØ Top Analysis and Data Sample for the Winter Conferences 2004," DØ Note 4419 (2004).

[44] M. Begel, "DØ Luminosity in Run 2: Online Bookkeeping," DØ Note 4137 (2003).

[45] T. Edwards et al., "Luminosity Constant for Dzero Run II," DØ Note 4496 (2004).

[46] G. Blazey, et al., "Run II Jet Physics," DØ Note 3750 (2000).

[47] "Jet Energy Scale and Resolutions," http://www-d0.fnal.gov/phys_id/jes/d0_private/jes.html. 
[48] U. Bassler and G. Bernardi, "Towards a Coherent Treatment of Calorimetric Energies: Missing Transverse Energy, Jets, E.M. Objects and the T42 Algorithm," DØ Note 4124 (2004).

[49] D. Whiteson and M. Kado, "Muon Isolation Studies," DØ Note 4070 (2003).

[50] M. Klute, "Measurement of the $t \bar{t}$ Cross Section at $\sqrt{s}=1.96 \mathrm{TeV}$ in $\mu$-Plus-Jets Events," DØ Note 4185 (2003).

[51] S. Anderson, " $t \bar{t}$ Topological Xsec in the Di-mu Channel,"

Talk to the General Top Quark Physics Group, http://www-d0.hef.kun.nl///fullAgenda.php?ida=a04193 (2004).

[52] E. Varnes, "Track Reconstruction Efficiency Measurement with Single Muons," DØ Note 4317 (2003).

[53] M. Angelou et al., "Top Trigger Efficiency Measurements and the top_trigger Package," DØ Note 4512 (2004).

[54] A.Garcia-Bellido, S. Lager, F. Rizatdinova, A. Schwartzman, and G. Watts, "Track Reconstruction Efficiency Measurement with Single Muons," DØ Note 4320 (2004).

[55] R. Brune, et al., "Root, an Object-Oriented Data Analysis Framework," http://root.cern.ch/root/htmldoc/TH1.html\#TH1:KolmogorovTest.

[56] G. J. Feldman and R. D. Cousins, Phys. Rev. D 57, 3873 (1998) [physics/9711021].

[57] F. Abe et al. [CDF Collaboration], Phys. Rev. Lett. 80, 2779 (1998) [hepex/9802017].

[58] D. Acosta et al. [CDF Collaboration], "Measurement of the t anti-t production cross section in p anti-p collisions at $\mathrm{s}^{* *}(1 / 2)=1.96-\mathrm{TeV}$ using dilepton events," [hep-ex/0404036] (2004). 